\title{
Embedded contact homology and Seiberg-Witten Floer cohomology IV
}

\author{
ClifFord Henry TAUBes
}

\begin{abstract}
This is the fourth of five papers that construct an isomorphism between the embedded contact homology and Seiberg-Witten Floer cohomology of a compact 3-manifold with a given contact 1 -form.
\end{abstract}

57R17; 57R57

\section{Introduction}

This is the fourth of a series of five papers whose purpose is to prove that embedded contact homology as defined by Michael Hutchings for a compact 3-manifold with contact 1-form is isomorphic to the manifold's Seiberg-Witten Floer cohomology. As described in the first paper [8, Section 4] of the series, this isomorphism involves two maps, one which maps generators of the embedded contact homology chain complex to generators of the Seiberg-Witten Floer cochain complex, and another which is used to identify the respective differentials. Let $M$ denote the manifold in question. The first of these maps assigns a solution on $M$ to a version of the Seiberg-Witten equations to certain data that is associated to a finite collection of Reeb orbits of the contact 1-form. This map is denoted by $\Phi^{r}$ in [8, Theorem 4.2]. The second map associates an instanton solution to the corresponding Seiberg-Witten equations on $\mathbb{R} \times M$ to data that is associated to certain collections of pseudoholomorphic curves in $\mathbb{R} \times M$. Theorem 4.3 of [8] denotes the latter map by $\Psi^{r}$. The maps $\Phi^{r}$ and $\Psi^{r}$ are constructed in the second paper [9] of this series. Theorems 4.2 and 4.3 of [8] respectively assert that $\Phi^{r}$ and $\Psi^{r}$ are surjective maps onto certain image sets. What follows in this paper proves that such is the case. Theorems 1.1 and 1.2 below make the formal statements to this effect. Theorem 4.3 of [8] makes additional assertions about instanton solutions to a certain perturbed version of the Seiberg-Witten equation on $\mathbb{R} \times M$. These extra assertions are first summarized by Proposition 8.1 and then proved. 


\section{1.a The image of the map $\Phi^{r}$}

The statement of Theorem 1.1 requires some background and notation that was introduced in the earlier papers in this series. The summary of this background constitutes the five part digression that follows.

Part 1 Use $a$ to denote the contact 1 -form. The manifold $M$ is oriented using as volume form $a \wedge d a$. The oriented 2-plane bundle $\operatorname{kernel}(a) \subset T M$ with its orientation given by $d a$ is denoted by $K^{-1}$. This bundle $K^{-1}$ will be viewed for the most part as a complex line bundle over $M$ with its orientation as a complex bundle the same as that given by $d a$. The first Chern class of $K^{-1}$ in $H^{2}(X ; \mathbb{Z})$ is denoted by $-c_{1}(K)$. The vector field on $M$ that generates the kernel of $d a$ and pairs with $a$ so as to equal 1 it called the Reeb vector field and it is denoted as $v$. Its integral curves are the Reeb orbits. They are oriented implicitly by $v$. Fix a homology class $\Gamma$ in $H_{1}(M ; \mathbb{Z})$ and let $\mathcal{Z}$ denote the set defined as follows: An element $\Theta \in \mathcal{Z}$ consists of a finite set of pairs of the form $(\gamma, m)$ with $\gamma$ a Reeb orbit and $m$ a positive integer. Require that distinct pairs from $\Theta$ have distinct Reeb orbit components, and require that the $\sum_{(\gamma, m) \in \Theta} m \gamma$ represents the class $\Gamma$. Given $L \geq 1$, use $\mathcal{Z}^{L}$ to denote the subset consisting of those $\Theta \in \mathcal{Z}$ which obey $\sum_{(\gamma, m)} m \ell_{\gamma} \leq L$ where $\ell_{\gamma}$ denotes the integral of the contact 1 -form along $\gamma$.

Part 2 Fix an almost complex structure $J: \operatorname{kernel}(a) \rightarrow \operatorname{kernel}(a)$ such that the bilinear form $d a(\cdot, J(\cdot))$ defines a positive definite inner product on $\operatorname{kernel}(a)$. Let $\gamma$ denote a given Reeb orbit. There is a disk $D \subset \mathbb{C}$ and an embedding $\varphi: S^{1} \times D \rightarrow M$ with the following properties: First, $\gamma$ appears as $S^{1} \times\{0\}$. Second,

$$
\begin{aligned}
& \text { - } \frac{2 \pi}{\ell_{\gamma}} \varphi^{*} a=\left(1-2 v|z|^{2}-\mu \bar{z}^{2}-\bar{\mu} z^{2}\right) d t+\frac{i}{2}(z d \bar{z}-\bar{z} d z)+O\left(|z|^{3}\right), \\
& \text { - } \frac{2 \pi}{\ell_{\gamma}} d a=i d z \wedge d \bar{z}-2(v z+\mu \bar{z}) d \bar{z} \wedge d t-2(v \bar{z}+\bar{\mu} z) d z \wedge d t+O\left(|z|^{2}\right), \\
& \text { - } \frac{\ell_{\gamma}}{2 \pi} v=\frac{\partial}{\partial t}+2 i(v z+\mu \bar{z}) \frac{\partial}{\partial z}-2 i(v \bar{z}+\bar{\mu} z) \frac{\partial}{\partial \bar{z}}+O\left(|z|^{2}\right) .
\end{aligned}
$$

Here, $v$ and $\mu$ are respectively real and complex valued functions on $S^{1}$. Here and in what follows, the circle $S^{1}$ is implicitly identified with $\mathbb{R} /(2 \pi \mathbb{Z})$ and $t \in \mathbb{R} /(2 \pi \mathbb{Z})$ is used to denote its affine coordinate. In these coordinates, the vector field $\partial / \partial z$ at $z=0$ pushes forward via $\varphi$ so as to generate the $+i$ eigenspace of $J$ on $\operatorname{kernel}(a) \otimes \mathbb{C}$. The pair $(\nu, \mu)$ are used to define the operator $\mathcal{L}$ on $C^{\infty}(\mathbb{R} ; \mathbb{C})$ given by

$$
\mathcal{L} z=\frac{i}{2} \frac{d}{d t} z+v z+\mu \bar{z}
$$


Let $t \rightarrow x(t)$ and $t \rightarrow y(t)$ denote the real and imaginary parts of a map $t \rightarrow z(t)$ in the kernel of $\mathcal{L}$. The latter obey

$$
\left(\begin{array}{l}
x(t) \\
y(t)
\end{array}\right)=U\left(\begin{array}{l}
x(0) \\
y(0)
\end{array}\right) \quad \text { where }\left.U\right|_{t} \in \operatorname{SL}(2 ; \mathbb{R}) \text { for each } t \in \mathbb{R}
$$

As $t$ varies in $[0,2 \pi]$, the map $\left.t \rightarrow U\right|_{t}$ defines a path in $\operatorname{SL}(2 ; \mathbb{R})$ starting at the identity. The Reeb orbit $\gamma$ is said to be nondegenerate when $\left|\operatorname{trace}\left(\left.U\right|_{2 \pi}\right)\right|>2$ or $\left|\operatorname{trace}\left(\left.U\right|_{2 \pi}\right)\right|<2$. In the former case, $\gamma$ is said to be hyperbolic, and in the latter case $\gamma$ is said to be elliptic. In the hyperbolic case, there is a homotopy of the map $\left.U\right|_{(.)}:[0,2 \pi] \rightarrow \operatorname{SL}(2 ; \mathbb{R})$ such that the $t=2 \pi$ element of each member of this homotopy has $\left|\operatorname{trace}\left(\left.U\right|_{(\cdot)}\right)\right| \geq 2$ and such that the final member is a rotation through $\pi k$ radians with $k \in \mathbb{Z}$. The integer $k$ is said to be the rotation number. In the elliptic case, there is a homotopy of $\left.U\right|_{(\cdot)}$ such that the $t=2 \pi$ element of each member of this homotopy is conjugate to $\left.U\right|_{2 \pi}$ and such that the end member is a rotation by angle $2 \pi \mathrm{R}$ with $\mathrm{R} \in \mathbb{R}$. The number $\mathrm{R}$ is the rotation number of $\gamma$ when $\gamma$ is elliptic. The $\bmod (2)$ reduction of $k$ is independent of $\varphi$ as is the $\bmod (\mathbb{Z})$ reduction of $\mathrm{R}$.

Fix $L \geq 1$. A contact form $a$ is said to be $L$-nondegenerate when the following three conditions are met: First, if $\Theta \in \mathcal{Z}$, then $\sum_{(\gamma, m) \in \Theta} m \ell_{\gamma} \neq L$. Second, if $\Theta \in \mathcal{Z}^{L}$, and $(\gamma, m) \in \Theta$, then $\gamma$ is nondegenerate. Third, if $\Theta \in \mathcal{Z}^{L}$ and $(\gamma, m) \in \Theta$ with $\gamma$ elliptic, then its rotation number $\mathrm{R}$ is such that $k \mathrm{R} \neq \mathbb{Z}$ for each $k \in\{1,2, \ldots, m\}$. The space of contact forms on $M$ has an open and dense set (in the $C^{\infty}$ topology) that contains only $L$-nondegenerate contact forms.

Part 3 Let $\Theta \in \mathcal{Z}$. Section 1.b of [9] associates a set $\mathfrak{C} \Theta$ and a subset $\mathfrak{C}^{*}$ to $\Theta$. To define these sets, focus first on a given $(\gamma, m) \in \Theta$. Introduce the vortex moduli space $\mathfrak{C}_{m}$ as defined in [9, Section 1.b]. The latter consists of equivalence classes of pairs $(A, \alpha)$ with $A$ being a connection on the product $\mathbb{C}$ bundle over $\mathbb{C}$ and with $\alpha$ a section of this bundle, thus a map to $\mathbb{C}$. This pair obeys the equations

- $* F_{A}=-i\left(1-|\alpha|^{2}\right)$.

- $\bar{\partial}_{A} \alpha=0$.

- $|\alpha| \leq 1$.

- The function $\left(1-|\alpha|^{2}\right)$ is integrable on $\mathbb{C}$ and $\int_{\mathbb{C}}\left(1-|\alpha|^{2}\right)=2 \pi m$.

The equivalence relation that defines $\mathfrak{C}_{m}$ posits $(A, \alpha) \sim\left(A-u^{-1} d u, u \alpha\right)$ when $u$ any smooth map from $\mathbb{C}$ to $U(1)$.

As noted in [9, Section 1.b], this space is the complex manifold, $\mathbb{C}^{m}$, with a nonstandard, nonflat Kahler metric. The latter defines a symplectic form and thus the Hamiltonian 
dynamical system that is defined using the time dependent Hamiltonian function

$$
\hbar=\frac{1}{4 \pi} \int_{\mathbb{C}}\left(2 v|z|^{2}+\left(\mu \bar{z}^{2}+\bar{\mu} z^{2}\right)\right)\left(1-|\alpha|^{2}\right) .
$$

The set $\mathfrak{C}_{(\gamma, m)}$ consists of the maps $\mathfrak{c}: S^{1} \rightarrow \mathfrak{C}_{m}$ that are closed, integral curves of the Hamiltonian vector field defined by $k$; thus solutions to the equation

$$
\frac{i}{2} \mathfrak{c}^{\prime}+\left.\nabla^{(1,0)} \mathfrak{h}\right|_{c}=0,
$$

where $\mathfrak{c}^{\prime}$ is shorthand for the $(1,0)$ part of $\mathfrak{c}_{*}(d / d t)$, and where $\nabla^{(1,0)} \mathfrak{h}$ denotes the $(1,0)$ part of the gradient of $h$. What is denoted below by $\mathfrak{C} \Theta$ is $\times_{(\gamma, m) \in \Theta} \mathfrak{C}_{(\gamma, m)}$; thus a typical element consists of a set $\left\{\mathfrak{c}_{\gamma} \in \mathfrak{C}_{(\gamma, m)}\right\}_{(\gamma, m) \in \Theta}$.

Let $\mathfrak{c}: S^{1} \rightarrow \mathfrak{C}_{m}$ denote a given map. Associate to $\mathfrak{c}$ the bundle $\mathfrak{c}^{*} T_{1,0} \mathfrak{C}_{m} \rightarrow S^{1}$. The pullback of the Riemannian connection on $T \mathfrak{C}_{m}$ defines a Hermitian connection on $S^{1}$. The map $\mathfrak{c}$ is said to be nondegenerate when the operator

$$
\zeta \rightarrow \frac{i}{2} \nabla_{t} \zeta+\left.\left(\nabla_{\zeta_{\mathbb{R}}} \nabla^{1,0} \hbar\right)\right|_{\mathfrak{c}}
$$

on $C^{\infty}\left(S^{1} ; \mathfrak{c}^{*} T_{1,0} \mathfrak{C}_{m}\right)$ has trivial kernel. The notation here is such that $\nabla_{t}$ denotes the covariant derivative of the aforementioned Hermitian connection. Also, $\left.\left(\nabla_{\zeta_{\mathbb{R}}} \nabla^{1,0} h\right)\right|_{\mathfrak{c}}$ denotes the covariant derivative at $\mathfrak{c}$ along the vector defined by $\zeta$ in $\left.T \mathfrak{C}_{m}\right|_{\mathfrak{c}}$ of the vector field $\nabla^{1,0} \mathfrak{h} \in C^{\infty}\left(\mathfrak{C}_{m} ; T_{1,0} \mathfrak{C}_{m}\right)$. The operator in (1-7) is symmetric and elliptic. Its spectrum is a discrete subset of $\mathbb{R}$ with finite multiplicities and no accumulation points. What is denoted here by $\mathfrak{C} \Theta *$ consists of the elements in $\mathfrak{C} \Theta$ of the form $\left\{\mathfrak{c}_{\gamma}\right\}(\gamma, m) \in \Theta$ with all $\mathfrak{c}_{\gamma}$ being nondegenerate.

Part 4 The definition of the Seiberg-Witten cochain complex requires the choice of a Riemannian metric on $M$. Such a metric should be chosen so that $* d a=2 a$ and such that $|a|=1$. Note that a metric of this sort defines an almost complex structure, $J$, on $\operatorname{kernel}(a)$ for which the bilinear form $d a(\cdot, J(\cdot))$ is positive definite and symmetric. Conversely, any such almost complex structure defines a unique metric on $M$ such that $* d a=2 a$ and $|a|=1$. This understood, a contact 1 -form $a$ and a chosen almost complex structure $J$ of the sort just described will be used implicitly to define the metric on $M$.

As noted in $\left[8\right.$, Section 3.c], the spinor bundle $\mathbb{S}$ for a given $S_{\text {pin }}{ }^{\mathbb{C}}$ structure decomposes as the orthogonal direct sum $E \oplus E K^{-1}$ where $E \rightarrow M$ is a complex, Hermitian line bundle, and where $K^{-1}$ is now viewed as a complex line bundle. These subbundles are the respective $+i$ and $-i$ eigenbundles for Clifford multiplication by the $1-$ form $a$. The first Chern class of $E$ can be used to classify the $\operatorname{Spin}^{\mathbb{C}}$ structure. 
This understood, choose $E$ so that its first Chern class is Poincaré dual to the homology class $\Gamma \in H_{1}(M ; \mathbb{Z})$ that was used in Part 1.

Let $\operatorname{Conn}(E)$ denote the space of smooth, Hermitian connections on $E$. The SeibergWitten equations used here require the choice of a real number $r \geq 1$ and then of a coclosed 1-form $\mu$. The corresponding Seiberg-Witten equations are for a pair $(A, \psi) \in \operatorname{Conn}(E) \times \mathbb{C}^{\infty}(M ; \mathbb{S}) ;$ they read

- $B_{A}-r\left(\psi^{\dagger} \tau \psi-i a\right)-i * d \mu+\frac{1}{2} B_{A_{K}}=0$.

- $D_{A} \psi=0$.

The notation here is as follows: First, $B_{A}$ denotes the metric Hodge star of the curvature 2-form of $A$. Second, $A_{K}$ is a fixed connection on $K^{-1}$ with harmonic curvature 2 -form. Third, $D_{A}$ denotes the Dirac operator on $C^{\infty}(M ; \mathbb{S})$ as defined using the metric's Levi-Civita connection and the connection $A_{K}+2 A$ on $\operatorname{det}(\mathbb{S})$. Finally, if $\eta$ and $\zeta$ are any given sections of $S$, then $\eta^{\dagger} \tau \zeta$ is the $\mathbb{C}$-valued 1 -form whose metric inner product with a 1 -form $b$ is $\eta^{\dagger} \operatorname{cl}(b) \zeta$ with $\mathrm{cl}(\cdot)$ here denoting the Clifford multiplication endomorphism.

The 1-form $\mu$ that appears in (1-8) is constrained to lie in a certain Banach space of smooth 1 -forms. The latter is denoted by $\Omega$ and is described briefly in [8, Section 3.d]. The norm on this space is called the $\mathcal{P}$-norm: it bounds all of the $C^{k}$ norms. In what follows, $\mu$ is assumed to come from $\Omega$ and have $\mathcal{P}$-norm less than 1 .

The group $C^{\infty}(M ; U(1))$ acts on $\operatorname{Conn}(E) \times \mathbb{C}^{\infty}(M ; \mathbb{S})$ as follows: A given map $u$ from $M$ to $U(1)$ sends a pair $(A, \psi)$ to $\left(A-u^{-1} d u, u \psi\right)$. Pairs related in this way are said to be gauge equivalent. Any given orbit under this action is said to be a gauge equivalence class. If $(A, \psi)$ obeys (1-8), then so does any pair in its gauge equivalence class. With $\mu$ fixed and $r \geq 1$ given, use $\mathcal{M}^{r}$ in what follows to denote the set of gauge equivalence classes of solutions to (1-8).

Associated to any connection on $E$ is a certain functional, this denoted in what follows by E. Its value on a given $A \in \operatorname{Conn}(E)$ is

$$
\mathrm{E}(A)=i \int_{M} a \wedge * B_{A} .
$$

This function is constant on any given gauge equivalence class.

With the stage set, fix $L \geq 1$ and assume that the contact form $a$ is $L$-nondegenerate. Let $\mathfrak{C} \mathcal{Z}^{L}$ denote the set of pairs $\left\{\mathfrak{C} \Theta: \Theta \in \mathcal{Z}^{L}\right\}$ and let $\mathfrak{C} \mathcal{Z}^{L *}$ denote the subset $\left\{\mathfrak{C} \Theta^{*}: \Theta \in \mathcal{Z}^{L}\right\}$. Fix a finite subset $\mathfrak{X} \subset \mathfrak{C Z}^{L *}$. Theorem 1.1 of [9] describes the 
following: For all large $r$ and any given $\mu \in \Omega$ with $\mathcal{P}$-norm bounded by 1 , an injective map

$$
\Phi^{r}: \mathfrak{X} \rightarrow \mathcal{M}^{r} \quad \text { with image in the set where } \mathrm{E}<2 \pi L .
$$

(Theorem 1.1 of [10] has more to say about this map.)

What follows is the promised surjectivity theorem for $\Phi^{r}$.

Theorem 1.1 Fix $L \geq 1$ and suppose that the contact 1 -form $a$ is $L$-nondegenerate. Fix an almost complex structure $J$ on the kernel of a so that $d a(\cdot, J(\cdot))$ defines a metric on the kernel of $a$. Use this data to define a metric on $M$. Suppose that $\mathfrak{C} \mathcal{Z}^{L *}=\mathfrak{C} \mathcal{Z}^{L}$. Then $\mathfrak{C} \mathcal{Z}^{L *}$ is a finite set. Moreover, there exists $\kappa \geq 1$ with the following significance: Fix $\mu \in \Omega$ and $r \geq \kappa$ so as to define $\mathcal{M}^{r}$ and the corresponding map $\Phi^{r}: \mathfrak{C Z}^{L} \rightarrow \mathcal{M}^{r}$. Then the image of $\Phi^{r}$ is the whole of the $\mathrm{E}<2 \pi L$ part of $\mathcal{M}^{r}$.

This theorem is proved in the upcoming Section 2.

\section{1.b The image of the map $\Psi^{r}$}

The statement of the upcoming theorem about the map $\Psi^{r}$ requires the three part digression that follows momentarily. This digression reintroduces notation and various notions from the previous papers in this series.

Part 1 The definition of $\Psi^{r}$ assumed that the chosen contact form $a$ was $L$-nondegenerate for all $L \geq 1$. The set of such forms is denoted by $\mathcal{N}_{M}$. The definition also took the almost complex structure from a certain set, $g_{a}$, of almost complex structures for $T(\mathbb{R} \times M)$. Fix $J \in \mathcal{I}_{a}$. Note that $J$ is unchanged by the constant translations along the $\mathbb{R}$ factor of $\mathbb{R} \times M$. It also maps $\partial / \partial s$ to $v$ and it maps $K$, the kernel of $a$, to itself. Moreover, $d a(\cdot, J(\cdot))$ is a Riemannian metric on the kernel of $a$. Thus $J$ can be used to define a metric for $M$ if it is understood that $a$ has norm 1 and that $d a=2 * a$. This is the metric to use in what follows, and to use when defining the Seiberg-Witten equations in (1-8).

Introduce $\mathcal{Z}_{\text {ech }}$ to denote the subset of those elements in $\mathcal{Z}$ that lack pairs of the form $(\gamma, m)$ with $\gamma$ hyperbolic and $m>1$. Fix two elements, $\Theta_{-}, \Theta_{+} \in \mathcal{Z}_{\text {ech }}$ and define $\mathcal{M}_{1}\left(\Theta_{-}, \Theta_{+}\right)$as follows: An element $\Sigma \in \mathcal{M}_{1}\left(\Theta_{-}, \Theta_{+}\right)$consists of a finite set of pairs of the form $(C, m)$ where $m$ is a positive integer and where $C$ is a $J$-pseudoholomorphic submanifold. These pairs are further constrained as follows: First, distinct pairs have distinct submanifold components. Second, $m=1$ unless $C$ is $\mathbb{R}$-invariant; thus $C$ is of the form $\mathbb{R} \times \gamma$ with $\gamma \subset M$ a Reeb orbit. To state the third property, let $\pi: \mathbb{R} \times M \rightarrow M$ denote the projection. Here is the third 
property: The formal sum $\sum_{(C, m) \in \Sigma} m \pi(C)$ defines a 2-cycle whose boundary is $\sum_{(\gamma, m) \in \Theta_{+}} m \gamma-\sum_{(\gamma, m) \in \Theta_{-}} m \gamma$. Finally, this cycle is homologous rel boundary to the image of a submanifold $Z \subset[0,1] \times M$ for which a certain integer $I\left(\Theta_{-}, \Theta_{+}, Z\right)$ is defined and equal to 1 . This integer $I\left(\Theta_{-}, \Theta_{+}, Z\right)$ is described in $[8,(2-9)]$ and the surrounding discussion in [8, Section 2.c].

Hutchings proves in [2] that $\mathcal{M}_{1}\left(\Theta_{-}, \Theta_{+}\right)$has the structure of a 1-dimensional manifold with a finite set of components. Moreover, each component is a copy of $\mathbb{R}$, this the orbit of any one of its members under the action of the group $\mathbb{R}$ that is induced by latter's action on $\mathbb{R} \times M$ as the constant translations of the $\mathbb{R}$ factor.

Part 2 Fix $r \geq 1$ and a coclosed 1-form $\mu$ for use in (1-8). The associated SeibergWitten equations on $\mathbb{R} \times M$ are equations for a map, $\mathfrak{d}$, from $\mathbb{R}$ into the space $\operatorname{Conn}(E) \times \mathbb{C}^{\infty}(M ; \mathbb{S})$. Write $\mathfrak{d}$ as the map $\left.s \rightarrow(A, \psi)\right|_{s}$ and these equations read

- $\frac{\partial}{\partial s} A+B_{A}-r\left(\psi^{\dagger} \tau \psi-i a\right)-i * d \mu+\frac{1}{2} B_{A_{K}}=0$.

- $\frac{\partial}{\partial s} \psi+D_{A} \psi=0$.

A solution $\mathfrak{d}$ is said to be an instanton when $\{\mathfrak{d}(s)\}_{s \in \mathbb{R}}$ converges as $s \rightarrow-\infty$ and also as $s \rightarrow \infty$ and both limits are solutions to (1-8).

Associated to any given map $\mathfrak{d}=(A, \psi): \mathbb{R} \rightarrow \operatorname{Conn}(E) \times \mathbb{C}^{\infty}(M ; \mathbb{S})$ is the elliptic operator, $\mathfrak{D}_{\mathfrak{d}}$, on $C^{\infty}\left(\mathbb{R} \times M ; i T^{*} M \oplus \mathbb{S} \oplus i \mathbb{R}\right)$ that sends a given section $(b, \eta, \phi)$ to the section with respective $i T^{*} M, \mathbb{S}$, and $i \mathbb{R}$ components

- $\frac{\partial}{\partial s} b+* d b-d \phi-2^{-1 / 2} r^{1 / 2}\left(\psi^{\dagger} \tau \eta+\eta^{\dagger} \tau \psi\right)$,

- $\frac{\partial}{\partial s} \eta+D_{A} \eta+2^{1 / 2} r^{1 / 2}(\mathrm{cl}(b) \psi+\phi \psi)$,

- $\frac{\partial}{\partial s} \phi+* d * b-2^{-1 / 2} r^{1 / 2}\left(\eta^{\dagger} \psi-\psi^{\dagger} \eta\right)$.

Here, $d$ denotes the exterior derivative along the $M$ factor of $\mathbb{R} \times M$.

Use $\mathbb{H}$ to denote the Hilbert space completion of the space of compactly supported sections over $\mathbb{R} \times M$ of $i T^{*} M \oplus \mathbb{S} \oplus i \mathbb{R}$ using the norm whose square is defined to be

$$
\|\mathfrak{b}\|_{\mathbb{H}^{2}}=\int_{\mathbb{R} \times M}\left(|\nabla b|^{2}+r|b|^{2}\right),
$$

where $\nabla$ is the covariant derivative that is defined on sections of $i T^{*} M \oplus \mathbb{S} \oplus i \mathbb{R}$ as follows: View $i T^{*} M \oplus i \mathbb{R}$ as $i T^{*}(\mathbb{R} \times M)$. This done, then $\nabla$ is the Levi-Civita covariant derivative on the $i T^{*}(\mathbb{R} \times M)$ factor. View the connection $A$ as a connection on the pullback $E \rightarrow \mathbb{R} \times M$ and likewise view $A_{K}$ as a connection on $K^{-1} \rightarrow \mathbb{R} \times M$. The latter with the Levi-Civita connection define a covariant derivative for $\mathbb{S} \rightarrow \mathbb{R} \times M$. 
The associated covariant derivative gives $\nabla$ on the $\mathbb{S}$ factor. Meanwhile, use $\mathbb{L}$ to denote the $L^{2}$ completion of the space of compactly supported sections over $\mathbb{R} \times M$ of $i T^{*} M \oplus \mathbb{S} \oplus i \mathbb{R}$. If the spinor component of $\mathfrak{d}$ is bounded, then $\mathfrak{D}_{\mathfrak{d}}$ defines a bounded, linear map from $\mathbb{H}$ to $\mathbb{L}$. In particular, such is the case when $\mathfrak{d}$ is an instanton.

To say more about this last case, digress momentarily and let $\mathfrak{c}=(A, \psi)$ denote any given pair in $\operatorname{Conn}(E) \times \mathbb{C}^{\infty}(M ; \mathbb{S})$. Associated to $\mathfrak{c}$ is the symmetric, elliptic operator $\mathfrak{L}_{\mathfrak{c}}$ on $C^{\infty}\left(M ; i T^{*} M \oplus \mathbb{S} \oplus i \mathbb{R}\right)$ that is defined so as to send any given $(b, \eta, \phi)$ to the section whose respective $i T^{*} M, \mathbb{S}$ and $i \mathbb{R}$ components are

- $* d b-d \phi-2^{-1 / 2} r^{1 / 2}\left(\psi^{\dagger} \tau \eta+\eta^{\dagger} \tau \psi\right)$,

- $D_{A} \eta+2^{1 / 2} r^{1 / 2}(\operatorname{cl}(b) \psi+\phi \psi)$,

- $* d * b-2^{-1 / 2} r^{1 / 2}\left(\eta^{\dagger} \psi-\psi^{\dagger} \eta\right)$.

The latter extends as an unbounded, self-adjoint operator on $L^{2}\left(M ; i T^{*} M \oplus \mathbb{S} \oplus i \mathbb{R}\right)$ with dense domain $L_{1}^{2}\left(M ; i T^{*} M \oplus \mathbb{S} \oplus i \mathbb{R}\right)$. The element $\mathfrak{c}$ is said to be nondegenerate when the kernel of $\mathfrak{L}_{\mathfrak{c}}$ is trivial.

Now consider an instanton $\mathfrak{d}$ and the associated operator $\mathfrak{D}_{\mathfrak{d}}$ in (1-13) viewed now as a bounded, linear map from $\mathbb{H}$ to $\mathbb{L}$. This map is Fredholm when both the $s \rightarrow-\infty$ and $s \rightarrow \infty$ limits of $\left.\mathfrak{d}\right|_{s}$ are nondegenerate. In this event, $\mathfrak{d}$ is said to be nondegenerate when its cokernel is $\{0\}$.

Suppose that $\mathfrak{c}_{-}$and $\mathfrak{c}_{+}$are nondegenerate solutions to (1-8). Let $\mathcal{M}_{1}\left(\mathfrak{c}_{-}, \mathfrak{c}_{+}\right)$denote the space of instanton solutions to (1-11) with the following properties: First, the $s \rightarrow-\infty$ limit of $\mathfrak{d}$ is $\mathfrak{c}_{-}$and the $s \rightarrow \infty$ limit is gauge equivalent to a configuration in the gauge orbit of $\mathfrak{c}_{+}$. Second, the Fredholm index of $\mathfrak{D}_{\mathfrak{d}}$ is equal to 1 .

Part 3 Fix $L \geq 1$ and assume the following about the contact 1 -form $a$ and the element $J \in \mathcal{J}_{a}$ :

- There is no element $\Theta \in \mathcal{Z}$ with $\sum_{(\gamma, m) \in \Theta} m \ell_{\gamma}=L$.

- Suppose that $\gamma$ is a Reeb orbit with $\ell_{\gamma}<L$. Then $\gamma$ has a tubular neighborhood map $\varphi: S^{1} \times D \rightarrow M$ as described in Part 2 of Section 1.a such that if $\gamma$ is hyperbolic with rotation number $\mathrm{R}$, then $(v, \mu)=$ $\left(\frac{1}{4} k, i \varepsilon e^{i k t}\right)$ with $\varepsilon>0$ but very small. Meanwhile, if $\gamma$ is elliptic, then its rotation number $\mathrm{R}$ is irrational. Furthermore,

(i) The pair $(\nu, \mu)=\left(\frac{1}{2} \mathrm{R}, 0\right)$.

(ii) The $\varphi^{*}$-pull back of $T^{1,0}(\mathbb{R} \times M)$ is spanned by $d s+i a$ and $\left(\ell_{\gamma} /(2 \pi)\right)(d z-i \mathrm{R} z d t)$. Moreover, these two forms are orthogonal and have norm $\sqrt{2}$. 
Let $\mathcal{Z}_{\text {ech }}^{L}=\mathcal{Z}_{\text {ech }} \cap \mathcal{Z}^{L}$, and define $\mathfrak{C} \mathcal{Z}_{\text {ech }}^{L}$ to be the set $\left\{\mathfrak{C} \Theta: \Theta \in \mathcal{Z}_{\text {ech }}^{L}\right\}$. It is a consequence of [8, Lemmas 2.1-2.4] that with (1-15) obeyed, then $\mathfrak{C Z}^{L}=\mathfrak{C} \mathcal{Z}^{L *}=$ $\mathfrak{C} \mathcal{Z}_{\text {ech }}^{L}$. In this case, $\mathfrak{C} \mathcal{Z}_{\text {ech }}^{L}=\mathcal{Z}_{\text {ech }}^{L}$ because if $\Theta \in \mathcal{Z}_{\text {ech }}^{L}$, then $\mathfrak{C} \Theta$ has but a single element, this the collection $\left\{\mathfrak{c}_{\gamma}: S^{1} \rightarrow \mathfrak{C}_{m}\right\}(\gamma, m) \in \Theta$ where each $\mathfrak{c}_{\gamma}$ if the constant map to the vortex in $\mathfrak{C}_{m}$ with $\alpha^{-1}(0)=\{0\}$.

With (1-15) understood, use $J$ to define the metric for $M$. Fix a 1 -form $\mu \in \Omega$ with $\mathcal{P}$-norm less than 1 for use in [9, (1-8), (1-9) and Theorem 1.1]. Use the latter theorem to define the large $r$ versions of $\Phi^{r}: \mathcal{Z}_{\text {ech }}^{L} \rightarrow \mathcal{M}^{r}$. Theorem 1.1 of [10] asserts that the image of $\Phi^{r}$ consists solely of nondegenerate solutions to (1-8).

Fix an ordered pair $\left(\Theta_{-}, \Theta_{+}\right)$from $\mathcal{Z}_{\text {ech }}^{L}$ and let $\mathfrak{c}_{-}$and $\mathfrak{c}_{+}$denote solutions to (1-8) whose gauge equivalence class defines the respective $\Phi^{r}$ images of $\Theta_{-}$and $\Theta_{+}$. Theorem 1.2 of [9] defines the map

$$
\Psi^{r}: \mathcal{M}_{1}\left(\Theta_{-}, \Theta_{+}\right) \rightarrow \mathcal{M}_{1}\left(\mathfrak{c}_{-}, \mathfrak{c}_{+}\right) .
$$

This is an injective and $\mathbb{R}$-equivariant map from $\mathcal{M}_{1}\left(\Theta_{-}, \Theta_{+}\right)$into the space of instantons with $s \rightarrow-\infty$ limit equal to $\mathfrak{c}_{-}$and with $s \rightarrow \infty$ limit gauge equivalent to $\mathfrak{c}_{+}$. Theorem 1.2 of [9] asserts that the image of $\Psi^{r}$ consists solely of nondegenerate instantons, and that it defines an embedding onto its image.

The stage is now set for the promised theorem here about $\Psi^{r}$.

Theorem 1.2 Fix $L \geq 1$ and then a contact 1-form $a \in \mathcal{N}_{M}$ and $J \in J_{a}$ for which (1-15) holds. There exists $\kappa \geq 1$ with the following significance: Use $a$ and $J$ to define the metric on $\mathbb{R}$. Fix $\mu \in \Omega$ with $\mathcal{P}$-norm less than 1 and $r \geq \kappa$ so as to define $\mathcal{M}^{r}$. Let $\Theta_{-}$and $\Theta_{+}$denote any two elements in $\mathcal{Z}_{\text {ech }}^{L}$, and let $\mathfrak{c}_{-}$and $\mathfrak{c}_{+}$denote solutions to (1-8) in the respective gauge equivalence classes $\Phi^{r}\left(\Theta_{-}\right)$and $\Phi^{r}\left(\Theta_{+}\right)$. Then the image of the map $\Psi^{r}$ depicted in (1-16) is the whole of $\mathcal{M}_{1}\left(\mathfrak{c}_{-}, \mathfrak{c}_{+}\right)$.

Sections 3-7 contain the proof of Theorem 1.2.

Theorem 4.3 of [8] also makes assertions about instanton solutions to a certain perturbed version of the Seiberg-Witten equations on $\mathbb{R} \times M$; these are the equations in [8, (3-6)]. Of particular concern here are the assertions made by Item (iii) from the first bullet of [8, Theorem 4.3]. These assertions are proved in Section 8.

\section{1.c Conventions and notation}

Notation and various constructions will be freely borrowed from $[8 ; 9 ; 10]$, the previous papers in this series. The reader should be familiar with their content. As in these 
previous papers, it is always the case that $c_{0}$ denotes a constant that is greater than 1 and is independent of what ever relevant data is under consideration. The precise value of $c_{0}$ can increase between subsequent appearances.

This paper, as with the previous papers in this series, makes use of a chosen "bump" function on $\mathbb{R}$; this is a smooth function $\chi: \mathbb{R} \rightarrow[0,1]$ that equals 1 on $\left(-\infty, \frac{5}{16}\right]$ and value 0 on $\left[\frac{7}{16}, \infty\right)$.

Acknowledgements Much is owed to Michael Hutchings for his many suggestions for improving the presentation.

This work was supported in part by the National Science Foundation.

\section{Proof of Theorem 1.1}

The theorem makes two distinct assertions. These are restated next as separate propositions.

Proposition 2.1 If the contact 1-form a from $\mathcal{N}_{M}$ then the following is true: Any given $L \geq 1$ version of the set $\mathfrak{C} \mathcal{Z}^{L}$ is finite if $\mathfrak{C} \mathcal{Z}^{L *}=\mathfrak{C} \mathcal{Z}^{L}$.

This proposition is proved in Section 2.b.

Proposition 2.2 Fix a contact 1-form $a$ and almost complex structure as in Theorem 1.1. Suppose that $L \geq 1$ is such that there are no elements $\Theta \in \mathcal{Z}$ with $\sum_{(\gamma, m) \in \Theta} m \ell_{\gamma}=L$. Suppose also that $\mathfrak{C} \mathcal{Z}^{L *}=\mathfrak{C Z}^{L}$ and that these sets are finite. There exists $\kappa \geq 1$ with the following significance: Fix $\mu \in \Omega$ with $\mathcal{P}$-norm less than 1 and $r \geq \kappa$ so as to define the map $\Phi^{r}$ with domain $\mathfrak{C Z}^{L}$. Then $\Phi^{r}\left(\mathfrak{C} \mathcal{Z}^{L}\right)$ contains the whole of the $\mathrm{E}<2 \pi L$ part of $\mathcal{M}^{r}$.

This last proposition is proved in Section 2.a.

\section{2.a Proof of Proposition 2.2}

The proof uses much the same strategy and constructions as used in the article $\mathrm{SW}=\mathrm{Gr}$ from [6] to prove an analogous statement about a map that assigns a solution to a version of the Seiberg-Witten equations on a compact, symplectic 4-manifold to data associated to a collection of pseudoholomorphic curves. Even so, the discussion that follows is meant to be more or less self-contained. 
Assume, to the contrary, that the proposition is false so as to derive a conclusion that is incompatible with this assumption. To this end, suppose that there exists a sequence $\left\{r_{n}, \mathfrak{c}_{n}=\left(A_{n}, \psi_{n}\right)\right\}_{n=1,2, \ldots}$ such that

- $\mathfrak{c}_{n}$ obeys the $r=r_{n}$ version of (1-8).

- $\mathrm{E}\left(A_{n}\right)<2 \pi L$.

- The gauge orbit of $\mathfrak{c}_{n}$ is not in the image of $\Phi^{r}$.

The eight parts of the following argument explain why the assumptions in (2-1) are not mutually compatible.

Part 1 The proof that the assumptions in (2-1) are not mutually compatible refers to somewhat stronger versions of some a priori bounds that are asserted by [7, Lemmas 2.2 and 2.3]. These are summarized by the upcoming lemma. This lemma introduces a convention that is used ubiquitously throughout this paper and also in $[8 ; 9 ; 10]$ by writing a section of $\mathbb{S}$ in terms of the decomposition of $\mathbb{S}$ as $E \oplus E K^{-1}$. When this is done, the given section $\psi$ is written as $(\alpha, \beta)$ with $\alpha$ denoting the part in $E$ and $\beta$ the part in $E K^{-1}$. The lemma also introduces a second convention: What is written as $\nabla_{A}$ denotes the covariant derivative on the relevant bundle that is defined using $A$ and, if necessary, the metric's Levi-Civita connection.

Lemma 2.3 There exists a constant $\kappa>1$ with the following significance: Fix any Spin ${ }^{\mathbb{C}}$ structure for $M$. Then, fix $\mu \in \Omega$ with $\mathcal{P}$-norm less that 1 and $r \geq \kappa$. Let $(A, \psi=(\alpha, \beta))$ denote a solution to the corresponding version of (1-8). Then

- $|\alpha| \leq 1+\kappa r^{-1}$.

- $|\beta|^{2} \leq \kappa r^{-1}\left(1-|\alpha|^{2}\right)+\kappa^{2} r^{-2}$.

- $\left|\nabla_{A} \alpha\right|^{2} \leq \kappa r\left(1-|\alpha|^{2}\right)+\kappa^{2}$.

- $\left|\nabla_{A} \beta\right|^{2} \leq \kappa\left(1-|\alpha|^{2}\right)+\kappa^{2} r^{-1}$.

In addition, for each $q \geq 1$, there exists a constant $\kappa_{q} \in(0, \infty)$ which is independent of $(A, \psi), r$ and $\mu$, and is such that

- $\left|\nabla_{A}^{q} \alpha\right|+r^{1 / 2}\left|\nabla_{A}^{q} \beta\right| \leq \kappa_{q} r^{q / 2}$.

Note that this lemma makes no apriori assumptions about $\mathrm{E}(A)$.

Proof Lemma 2.3 [7, Lemmas 2.2-2.4] assert the first two items and the final item. To establish the third and fourth items, differentiate the equation $D_{A}^{2} \psi=0$ and copy 
the manipulations done in Section 2e of the article $\mathrm{SW} \Rightarrow \mathrm{Gr}$ from [6] to find $r$ and $(A, \psi)$ independent, positive constants $c_{1}, c_{2}$, and $c_{3}$ such that the function

$$
h=\left(\left|\nabla_{A} \alpha\right|^{2}+r|\nabla \beta|^{2}\right)+c_{1} r^{2}|\beta|^{2}-c_{2} r\left(1-|\alpha|^{2}\right)-c_{3}
$$

obeys

$$
\frac{1}{2} d^{\dagger} d h+c_{0}^{-1} r h \leq 0
$$

It follows as a consequence of the maximum principle that

$$
\left|\nabla_{A} \alpha\right|^{2}+r|\nabla \beta|^{2} \leq c_{0} r\left(1-|\alpha|^{2}\right)+c_{0}^{\prime},
$$

where $c_{0}^{\prime}$ is independent of both $r$ and $(A, \psi)$.

Part 2 It was argued in [7, Section 6.d] that there exists the following: First, a finite set $\Theta=\{(\gamma, m)\}$ of pairs such that $\gamma$ is a Reeb orbit and $m$ is a positive integer. Moreover, distinct pairs from $\Theta$ have distinct Reeb orbit components. Second, a subsequence of $\left\{\mathfrak{c}_{n}=\left(A_{n}, \psi_{n}=\left(\alpha_{n}, \beta_{n}\right)\right)\right\}_{n=1,2, \ldots}$ (hence renumbered consecutively) such that $\left\{\alpha_{n}^{-1}(0)\right\}_{n=1,2, \ldots}$ converges in a technical sense to the current represented by $\sum_{(\gamma, m)} m \gamma$.

To elaborate on this, fix $\delta>0$. It was argued in [7, Section 6.d] that if $n$ is large, then $\left|\alpha_{n}\right|>1-\delta$ at distances greater than $\delta$ from $\bigcup_{(\gamma, m) \in \Theta} \gamma$. Meanwhile, the behavior a solution near a Reeb orbit from $\Theta$ is as follows: Fix $(\gamma, m) \in \Theta$ and view a neighborhood of $\gamma$ using the coordinates $S^{1} \times D$ used in (1-1). If the index $n$ is large, then $\alpha_{n}$ vanishes on each disk $\{t\} \times D$ with multiplicity $m$ in the sense that $\alpha_{n} /|\alpha|$ has degree $m$ on the circles in $D$ about the origin with radius $\delta$ or greater. Lemmas 6.1 and 6.3 of [7] imply the following: Set $r=r_{n}$. There exist at most $m$ disjoint disks in $D$ of radius at most $c_{0} r^{-1 / 2}$ that contain the set in $\{t\} \times D$ where $\left|\alpha_{n}\right|<1-\delta$. Moreover, any such disk must contain at least one zero of $\alpha_{n}$, and $\alpha_{n} /\left|\alpha_{n}\right|$ has positive degree about each such disk. These lemmas imply somewhat more: There exists a set of at most $m$ disjoint disks in $D$ of radius $\delta r^{-1 / 2}$ such that each disk contains a zero of $\alpha_{n}$ in $\{t\} \times D$ and such that $\left|\alpha_{n}\right| \geq \delta / c_{0}$ on the complement of these disks. Moreover, $\alpha_{n} /\left|\alpha_{n}\right|$ has positive degree on each such disk.

Suppose that $\alpha_{n}$ vanishes at $\left(t, z_{t}\right) \in S^{1} \times D$ with positive degree. Parametrize the integral curve of $v$ through $\left(t, z_{t}\right)$ via $[0,2 \pi]$ as the map $x \rightarrow\left(t+x, z_{t}(x)\right)$. It follows from [7, Lemma 6.5] that $\alpha_{n}$ has a zero with distance at most $c_{0} r^{-1 / 2} x$ from $\left(t+x, z_{r}(x)\right)$ when $|x| \leq 1 / c_{0}$. It follows from this and the assumed nondegeneracy of the Reeb orbits that $\left|z_{t}(x)\right| \leq c_{0} r^{-1 / 2}$ when $n$ is large. To elaborate, suppose first that $m=1$. Then letting $x$ move from 0 to $2 \pi$ shows that the integral curve of $v$ through $\left(t, z_{t}\right)$ comes back to $t$ at a point with distance at most $c_{0} r^{-1 / 2}$ from $z_{t}$. Such 
a return is not possible unless $\left|z_{t}\right| \leq c_{0} r^{-1 / 2}$ given that $\gamma$ is nondegenerate. Note that $\gamma$ is nondegenerate because $a \in \mathcal{N}_{M}$.

In the case $m>1$, then $z_{t}(2 \pi)$ must have distance at most $c_{0} r^{-1 / 2}$ from either $z_{t}$ or from some other positive degree zero of $\alpha_{n}$ in $\{t\} \times D$. This is impossible if $\left|z_{t}\right|<c_{0} r^{-1 / 2}$ if $\gamma$ is hyperbolic, or if $\gamma$ is $m$-elliptic. One or the other of these describes $\gamma$ when $a \in \mathcal{N}_{M}$.

Part 3 Fix an identification over $S^{1} \times D$ between the bundle $E$ and the complex line bundle $\left(S^{1} \times D\right) \times \mathbb{C}$. Set $r=r_{n}$ and pull back $\left(A_{n},\left(\alpha_{n}, \beta_{n}\right)\right)$ by the rescaling map $\hat{r}_{\gamma}^{-1}$ that acts as $z \rightarrow r_{\gamma}^{-1 / 2} z$. Denote this pullback as $\left(A_{0 *} d t+A_{*},\left(\alpha_{*}, r_{\gamma}^{-1 / 2} \beta_{*}\right)\right)$ with $A_{*}$ at any given $t \in S^{1}$ a connection on the trivial bundle over the rescaled $D$, and where $\alpha_{*}$ and $\beta_{*}$ are sections of this bundle. Fix $R \geq 1$ and take $n$ large enough so as to guarantee that the disk of radius $R$ about the origin in $\mathbb{C}$ is in the rescaled version of $D$. It follows from (1-8) and from Lemma 2.3 that

- $* F_{A_{*}}+i\left(1-\left|\alpha_{*}\right|^{2}\right)=\mathfrak{e}_{0}$,

- $\bar{\partial}_{A_{*}} \alpha_{*}=\mathfrak{e}_{1}$,

on this $R$ disk in $\mathbb{C}$. Here, $F_{A_{*}}$ denotes the curvature of $A_{*}$ on $D$ at the given $t \in S^{1}$. Meanwhile, $\left|\mathfrak{e}_{0}\right| \leq c_{0} r^{-1 / 2}$ and $\left|\mathfrak{e}_{1}\right| \leq c_{0} r^{-1 / 2}$ when $n$ is large. As noted in [7, Lemma 6.5], what is written in (2-5) and what is said in Lemma 2.3 have the following consequence: Fix $\delta>0$ and $k \in \mathbb{Z}$. Then there exists $R_{\delta} \geq 1$ such that if $R \geq R_{\delta}$, and if $n$ is sufficiently large, there exits for each $t \in S^{1}$ a solution $\left.(A, \alpha)\right|_{t}$ of (1-4) on $\mathbb{C}$ whose restriction to where $|z| \leq R$ has $C^{k}$ distance less than $\delta$ from $\left.\left(A_{*}, \alpha_{*}\right)\right|_{t}$.

The middle item in (2-1), the fourth item in (1-4) and this last conclusion imply the following:

Lemma 2.4 The set $\Theta$ is such that $\sum_{(\gamma, m) \in \Theta} m \ell_{\gamma} \leq L$.

This lemma associates an element $\Theta \in \mathcal{Z}^{L}$ to a subsequence of $\left\{\left(A_{n}, \psi_{n}\right)\right\}_{n=1,2, \ldots}$.

Part 4 Fix $(\gamma, m) \in \Theta$. As noted just prior to Lemma 2.4, there exists for each $t \in S^{1}$, a solution $\left.(A, \alpha)\right|_{t}$ on $\mathbb{C}$ to the integer $m$ version of (1-4) that is obtained as a limit of rescalings of a subsequence of $\left\{\left(A_{n}, \alpha_{n}\right)\right\}_{n=1,2, \ldots}$. What follows says something about the $t$-dependence of $\left.(A, \alpha)\right|_{t}$.

Lemma 2.5 There exists a map $\mathfrak{c}=(A, \alpha): S^{1} \rightarrow \mathfrak{C}_{m}$ that obeys (1-6), and there exists a subsequence of $\left\{\left(A_{n}, \psi_{n}\right)\right\}_{n=1,2, \ldots}$ with the following property: Renumber 
the subsequence consecutively from 1 . Fix $\delta>0, T \geq 1$ and an integer $k$. If $n$ is sufficiently large, there exists, for each $t \in S^{1}$, a map from the $|z| \leq T$ part of $\mathbb{C}$ to $U(1)$ such that the $\left(A_{n}, \psi_{n}\right)$ version of $\left.\left(A_{*}-u_{*}^{-1} d u_{*}, u_{*} \alpha_{*}\right)\right|_{t}$ has $C^{k}$ distance $\delta$ or less from $\left.(A, \alpha)\right|_{t}$ on the disk in $\mathbb{C}$ where $|z| \leq T$.

This lemma assigns to each pair $(\gamma, m) \in \Theta$ a map $\mathfrak{c}_{\gamma}: S^{1} \rightarrow \mathfrak{C}_{m}$ that solves (1-6) and in doing so, it associates an element $x \in \mathfrak{C} \Theta^{L}$ to a subsequence of $\left\{\left(A_{n}, \psi_{n}\right)\right\}_{n=1,2, \ldots}$.

Proof of Lemma 2.5 Fix $0 \in \mathbb{R} / 2 \pi \mathbb{Z}=S^{1}$ and define an isomorphism between the bundle $E \rightarrow\{0\} \times D$ to the product bundle by using $A_{n}$ to parallel transport a given section over $\{0\} \times 0$ along the radial geodesics. Then use parallel transport by the connection $A_{n}$ along the constant $z \in D$ arcs to extend this isomorphism as one from $E$ over $(0-\pi, 0+\pi) \times D \subset S^{1} \times D$ to the product bundle. Let $\theta$ denote the corresponding product connection. Pull back $\left(A_{n},\left(\alpha_{n}, \beta_{n}\right)\right)$ and this product connection by the rescaling map. Given the choice of product structure, the pullback of $A_{n}$ has component $A_{0 *}=0$. Meanwhile, the connection $A_{*}$ can be written as $\theta+\frac{1}{2}\left(a_{*} d \bar{z}-\bar{a}_{*} d z\right)$ where $a_{*}$ is a $\mathbb{C}$-valued function given $t=0$ by the integral

$$
\left.a_{*}\right|_{z}=\left.i z \int_{0}^{1} * F_{A_{*}}\right|_{s z} s d s
$$

Likewise, $\alpha_{*}$ and $\beta_{*}$ are to be viewed as $\mathbb{C}$-valued functions. It follows from (1-8) that the derivatives of the large $n$ versions of $a_{*}$ and $\alpha_{*}$ at points where $|z| \leq T$ obey

$$
\begin{aligned}
& \text { - } 2^{-1 / 2} \frac{\partial}{\partial t} a_{*}=2^{1 / 2} \bar{\alpha}_{*} \beta_{*}-2 i(v z+\mu \bar{z}) 2^{-1 / 2}\left(1-\left|\alpha_{*}\right|^{2}\right)+\mathfrak{e}_{2}, \\
& \text { - } \frac{\partial}{\partial t} \alpha_{*}=-2 \partial_{A_{*}} \beta_{*}-2 i(v z+\mu \bar{z}) \partial_{A_{*}} \alpha_{*}+\mathfrak{e}_{3},
\end{aligned}
$$

with $\left|\mathfrak{e}_{2,3}\right| \leq c_{0} r^{-1 / 2}$. Note that $r^{1 / 2}\left|\beta_{*}\right|+\left|\nabla_{A_{*}} \beta_{*}\right| \leq c_{0}$; this a consequence of Lemma 2.3.

Each $\left(A_{n}, \psi_{n}\right)$ has its version of $\left(a_{*}, \alpha_{*}\right)$, this now denoted by $\left(a_{* n}, \alpha_{* n}\right)$. Given the last item in Lemma 2.3 and given what just said about the $\beta_{*}$ terms in (2-7), this equation implies the following: For any given positive integer $k$ and $T \geq 1$, the sequence $\left\{\left(a_{* n}, \alpha_{* n}\right)\right\}_{n=1,2, \ldots}$ is uniformly Lipschitz as a map from the interval $[-\pi, \pi]$ into the Banach space of $k$-times differentiable maps from the disc of radius $T$ in $\mathbb{C}$ to $\mathbb{C} \times \mathbb{C}$. There is, as a consequence, a convergent subsequence. Taking diagonal subsequences, finds a convergent subsequence of $\left\{\left(a_{n}, \alpha_{* n}\right)\right\}_{n=1,2, \ldots}$ whose limit is a Lipschitz map, $(a, \alpha)$, from the interval $[-\pi, \pi]$ into the Frechet space $C^{\infty}(\mathbb{C} ; \mathbb{C} \times \mathbb{C})$ with the three properties that follow. 
Property 1 The limit pair $c=\left(A=\theta+\frac{1}{2}(a d \bar{z}-\bar{a} d z), \alpha\right)$ obeys the vortex equations, (1-4), at each $t \in[-\pi, \pi]$, and so defines a point in the vortex moduli space $\mathfrak{C}_{m}$.

Property 2 The $t=\pi$ version of $(A, \alpha)$ is gauge equivalent to the $t=-\pi$ version. Thus, $\mathfrak{c}$ defines a Lipschitz map from $S^{1}$ into $\mathfrak{C}_{m}$.

To state the third property, recall that the $(1,0)$ part of the complexified tangent space to $\mathfrak{C}_{m}$ at any given element $\mathfrak{c}=(A, \alpha)$ is canonically isomorphic to the $L^{2}$ kernel of the operator $\vartheta_{\mathfrak{c}}$ on $C^{\infty}(\mathbb{C} ; \mathbb{C} \oplus \mathbb{C})$ that sends pair $(q, \eta)$ to

$$
\vartheta_{\mathfrak{c}}(q, \eta)=\left(\partial q+2^{-1 / 2} \bar{\alpha} \eta, \bar{\partial}_{A} \eta+2^{-1 / 2} \alpha q\right) .
$$

The third property refers to the function $\chi: \mathbb{R} \rightarrow[0,1]$ from [9]; it equals 1 on $\left(-\infty, \frac{5}{16}\right]$ and vanishes on $\left[\frac{7}{16}, \infty\right)$. Here is the third property:

Property 3 Fix $t \in[-\pi, \pi]$ and let $\left.\Pi\right|_{t}$ denote the $L^{2}(\mathbb{C} ; \mathbb{C} \oplus \mathbb{C})$ orthogonal projection onto the space of $L^{2}$ solutions to the $\left.(A, \alpha)\right|_{t}$ version of (2-8). For any given $T \geq 1$, use $\chi^{T}$ to denote the function $z \rightarrow \chi\left(T^{-1}|z|\right)$ on $\mathbb{C}$. Let $\lambda=$ $-2 i(v z+\mu \bar{z})\left(2^{-1 / 2}\left(1-|\alpha|^{2}\right), \partial_{A} \alpha\right)$. Then

$$
\lim _{\Delta \rightarrow 0} \frac{1}{\Delta} \Pi_{t}\left(\left.\chi^{T}(a, \alpha)\right|_{t+\Delta}-\left.\chi^{T}(a, \alpha)\right|_{t}\right)=\Pi_{t}\left(\left.\chi^{R} \lambda\right|_{t}\right)+\mathfrak{e},
$$

where $|\mathfrak{e}| \leq c_{0} T^{-1}$.

Note that (2-9) follows from the fact that the term $\left(2^{1 / 2} \bar{\alpha} \beta_{*},-2 \partial_{A_{*}} \beta_{*}\right)$ in (2-7) can be written as $\vartheta_{\mathfrak{c}_{*}}^{\dagger}\left(0,2 \beta_{*}\right)$ where $\vartheta_{\mathfrak{c}_{*}}$ is the $\mathfrak{c}_{*}=\left(A_{*}, \alpha_{*}\right)$ version of the operator in (2-8) and where $\vartheta_{\mathfrak{c}_{*}}^{\dagger}$ denotes the formal, $L^{2}$ adjoint of $\vartheta_{\mathfrak{c}_{*}}$.

What with the second bullet in (2-16), Property 3 implies that $\mathfrak{c}=(A, \alpha)$ defines a map from $S^{1}$ into $\mathfrak{C}_{m}$ that obeys (1-6).

Part 5 Parts 3 and 4 give an element in $\mathfrak{C Z}^{L}$ that characterizes a part of the limiting behavior of a subsequence of the original sequence $\left\{\left(A_{n}, \psi_{n}\right)\right\}_{n=1,2, \ldots}$. Relabel this subsequence consecutively from 1 . Meanwhile, this element in $\mathfrak{C Z}^{L}$ consists of the element $\Theta \in \mathcal{Z}^{L}$ and a corresponding set $\left\{\mathfrak{c}_{\gamma}: S^{1} \rightarrow \mathfrak{C}_{m}\right\}(\gamma, m) \in \Theta$ where each $\mathfrak{c}_{\gamma}$ solves the version of (1-6) defined by $\gamma$. Let $\left(A^{*}, \psi^{*}\right)$ denote the result of applying the constructions in $\left[9\right.$, Section 3.a] to the data $\left\{\left(\mathfrak{c}_{\gamma}, \zeta_{\gamma}=0\right)\right\}(\gamma, m) \in \Theta$. This is to say that $\left(A^{*}, \psi^{*}\right)$ is the $\mathfrak{J}=\left\{\left(\mathfrak{c}_{\gamma}, \zeta_{\gamma}=0\right)\right\}(\gamma, m) \in \Theta$ version of the pair that is defined in [9, Step 4 of Section 3.a].

What follows describes a gauge transformation of each sufficiently large $n$ version of $\left(A_{n}, \psi_{n}\right)$ that make the latter pair close on the whole of $M$ to the large $r$ versions of $\left(A^{*}, \psi^{*}\right)$. The following lemma summarizes: 
Lemma 2.6 There exists $\kappa>1$ with the following significance: Fix $\delta>0$ and take $n$ very large. There exists a smooth map $u: M \rightarrow U(1)$ such that $\left(A_{n}-u^{-1} d u, u \psi_{n}\right)$ can be written as $\left(A^{*}+2^{1 / 2} r^{1 / 2} b, \psi^{*}+\eta\right)$ where $(b, \eta)$ obey $|b|+|\eta| \leq \kappa \delta$ and $|\nabla b| \leq \kappa r^{1 / 2}$.

Proof of Lemma 2.6 The required gauge transformation is constructed in seven steps.

Step 1 Recall that $\left(A^{*}, \psi^{*}\right)$ is constructed using trivializations of $E$ over the sets $\left\{U_{\gamma}\right\}_{(\gamma, m) \in \Theta}$ and over $U_{0}=M-\bigcup_{(\gamma, m) \in \Theta} U_{\gamma}^{\prime}$. Here, $U_{\gamma}^{\prime}$ corresponds to the subset of points in $\gamma$ 's version of $S^{1} \times D$ with $|z| \leq \rho_{*}$. The pair $\left(A^{*}, \psi^{*}\right)$ over $U_{0}$ is defined by fixing a trivialization of $E$ over $U_{0}$ as $U_{0} \times \mathbb{C}$, and then taking $\psi^{*}=\left(1_{\mathbb{C}}, 0\right)$ and taking $A^{*}$ to be the product connection $A_{I}$. Here, $1_{\mathbb{C}}$ denotes the section of $U_{0} \times \mathbb{C}$ that assigns each point 1 in $\mathbb{C}$. Meanwhile, the large $n$ versions of $\left(A_{n}, \psi_{n}=\left(\alpha_{n}, \beta_{n}\right)\right)$ are such that $\left|\alpha_{n}\right| \geq 1-\delta$ over $U_{0}$. As a consequence, there exists a map $u_{0}: U_{0} \rightarrow S^{1}$ such that $u_{0} \alpha_{n}=\left|\alpha_{n}\right| 1_{\mathbb{C}}$ when $n$ is large. What with the second item in Lemma 2.3, this implies that $u_{0} \psi=\psi^{*}+\eta_{0}$ where $\left|\eta_{0}\right| \leq \delta$.

To continue, write $A_{n}-u_{0}^{-1} d u_{0}=A_{I}+\widehat{a}$. Then $\left|\nabla_{A} \alpha\right|^{2}=|d| \alpha \|^{2}+|\alpha|^{2}|\widehat{a}|^{2}$. It follows from the third item in Lemma 2.3 that $|\widehat{a}| \leq c_{0} r^{1 / 2} \delta$; and so $b_{0}=2^{-1 / 2} r^{-1 / 2} \widehat{a}$ obeys $\left|b_{0}\right| \leq c_{0} \delta$. Likewise, Lemma 2.3 implies that $\left|\nabla b_{0}\right| \leq \kappa r^{1 / 2}$.

Step 2 Focus attention on a pair $(\gamma, m) \in \Theta$. The pair $\left(A^{*}, \psi^{*}\right)$ is given on $U_{\gamma}$ by what is written in [9, (3-3)]. Fix $T \gg 1$ so that the both the large $n$ versions of $\left|\alpha_{n}\right|$ and $\left|\alpha^{\zeta=0, r}\right|$ are greater than $1-\delta$ at points with $|z| \geq \frac{1}{8} r^{-1 / 2} T$. The trivialization of $E$ over this part of $U_{\gamma}$ writes $\alpha^{0, r}=\left|\alpha^{0, r}\right| z^{m} /|z|^{m}$. Meanwhile, there is a map, $u_{\gamma}$, that is defined on this same part of $U_{\gamma}$ such that $u_{\gamma} \alpha_{n}=\left|\alpha_{n}\right| z^{m} /|z|^{m}$. This understood, the argument given in Step 1 can be repeated to prove that $\left(A_{n}-u_{\gamma}^{-1} d u_{\gamma}, u_{\gamma} \psi_{n}\right)=$ $\left(A^{*}+2^{1 / 2} r^{1 / 2} b_{\gamma}, \psi^{*}+\eta_{\gamma}\right)$ where $\left|b_{\gamma}\right|+\left|\eta_{\gamma}\right| \leq c_{0} \delta$ and where $\left|\nabla b_{\gamma}\right| \leq \kappa r^{1 / 2}$.

By virtue of the fact that $\alpha_{n}$ is a section of $E$, there exists an extension of $u_{\gamma}$ as a map from the whole of $U_{\gamma}$ to $U(1)$. Fix such an extension and denote it by $u_{\gamma 0}$.

Step 3 Suppose that there exists an extension of $u_{\gamma}$ from the $|z| \geq \frac{1}{2} r^{-1 / 2} T$ part of $U_{\gamma}$ to the whole of the $|z| \leq \frac{1}{2} r^{-1 / 2} T$ part of $U_{\gamma}$ such that $\left(A_{n}-u_{\gamma} d u_{\gamma}, u_{\gamma} \psi_{n}\right)$ can be written as $\left(A^{*}+A_{0 \gamma} d \tau+2^{1 / 2} r^{1 / 2} b_{\gamma}^{\prime}, \psi^{*}+\eta_{\gamma}\right)$ with $\left|b_{\gamma}^{\prime}\right|+\left|\eta_{\gamma}\right| \leq c_{0}\left(\delta+\left|\zeta_{\gamma}\right|\right)$ and $\left|\nabla b_{\gamma}^{\prime}\right| \leq c_{0} r^{1 / 2}$ when $n$ is large; and with $\left|A_{0 \gamma}\right| \leq c_{R}$ and $\left|\nabla A_{0 \gamma}\right| \leq r^{1 / 2} c_{T}$ where $c_{T}$ depends only on $T$ and not $n$. Given what is said in Steps 1 and 2, an extension of this sort implies the assertion of Lemma 2.6. The existence of such an extension is proved in the forthcoming Steps 4-7. An extension of this sort is deemed to be admissible. 
The notation used in Steps 4-7 comes from the proof of Lemma 2.5. In particular, the arguments use the $r=r_{n}$ versions of the rescaling map $\widehat{r}_{\gamma}$ from Part 3 so as to consider the various $n$-dependent entities on the product of $S^{1}$ with the disk in $\mathbb{C}$ where $|z| \leq T$.

Step 4 Introduce the Lipschitz map $(a, \alpha):[-\pi, \pi] \rightarrow C^{\infty}(\mathbb{C} ; \mathbb{C} \oplus \mathbb{C})$ that is constructed in the proof of Lemma 2.5. Let $\theta$ denote the product connection on the product bundle over $[-\pi, \pi] \times \mathbb{C}$. The pair $(a, \alpha)$ defines the pair $\left(\theta+\frac{1}{2}(a d \bar{z}-\bar{a} d z), \alpha\right)$ of connection on the trivial bundle over $[-\pi, \pi] \times \mathbb{C}$ and section of this bundle. This pair satisfies the vortex equations on $\mathbb{C}$ at each $t \in[-\pi, \pi]$ and the induced 1 -parameter family of connection along the $\mathbb{C}$ factor of $[-\pi, \pi]$ and section projects to $\mathfrak{C}_{m}$ as the map $\mathfrak{c}_{\gamma}$. Of particular interest here is the existence of a smooth gauge transformation, $u: \mathbb{C} \rightarrow U(1)$, such that $\left.a\right|_{t=\pi}=\left.a\right|_{t=-\pi}-2 u^{-1} \bar{\partial} u$ and $\left.\alpha\right|_{t=\pi}=\left.u \alpha\right|_{t=-\pi}$. As $\mathbb{C}$ is simply connected, the map $u$ can be written as $e^{p}$ where $p: \mathbb{C} \rightarrow i \mathbb{R}$ is a smooth map with ip $\left.\right|_{z=0} \in[0,2 \pi)$.

Introduce $\chi^{0}$ to denote the function $t \rightarrow \chi(t)$ on $[-\pi, \pi]$. Let $\widehat{u}=e^{\left(1-\chi^{0}\right) p}$. The pair

$$
\theta+\widehat{u}^{-1} \frac{\partial}{\partial t} \widehat{u} d t+\frac{1}{2}\left(\left(a+2 \widehat{u}^{-1} \bar{\partial} \widehat{u}\right) d \bar{z}-\left(\bar{a}-2 \widehat{u}^{-1} \partial \widehat{u}\right) d z\right) \quad \text { and } \quad \hat{u} \alpha
$$

defines a connection on the product bundle over $S^{1} \times \mathbb{C}$ and section of this bundle. Although smooth with respect to variations along the $\mathbb{C}$ factor of $S^{1} \times \mathbb{C}$, the coefficients of the connection and the section may only be Lipschitz with regards to their variation along the $S^{1}$ factor of $S^{1} \times \mathbb{C}$. In any event, the induced 1 -parameter family of connection along the $\mathbb{C}$ factor and the section project so as to give a Lipschitz map $\mathfrak{c}_{\gamma}: S^{1} \rightarrow \mathbb{C}$.

Step 5 This step defines an $\left(A_{n}, \psi_{n}\right)$ analog of (2-10). To start, recall from the proof of Lemma 3.5 that the $\left(A_{n}, \alpha_{n}\right)$ version of what is written in (2-5)-(2-7) as $\left(a_{*}, \alpha_{*}\right)$ obeys $\left.a_{*}\right|_{t=\pi}=\left.a_{*}\right|_{t=-\pi}-2 u_{*}^{-1} \bar{\partial} u_{*}$ and $\left.\alpha_{*}\right|_{t=\pi}=\left.u_{*} \alpha_{*}\right|_{t=-\pi}$ where the $\left(A_{n}, \psi_{n}\right)$ version of $u_{*}$ is a smooth map from the $|z| \leq R$ portion of $\mathbb{C}$ to $i \mathbb{R}$. As this disk is simply connected, the map $u_{*}$ can be written as $e^{p_{*}}$ where $p_{*}$ is a map from the $|z| \leq T$ part of $\mathbb{C}$ to $i \mathbb{R}$ with the property that $\left.i p_{*}\right|_{z=0} \in[0,2 \pi)$. Integrating the top equation in (2-7) finds that $\left|d p_{*}\right| \leq c_{0}$. As a consequence, $\left|p_{*}\right| \leq c_{0} T$. Define $\widehat{u}_{*}=e^{\left(1-\chi^{0}\right) p_{*}}$. Then the pair

$$
\text { 1) } \theta+\widehat{u}_{*}^{-1} \frac{\partial}{\partial t} \widehat{u}_{*} d t+\frac{1}{2}\left(\left(a_{*}+2 \widehat{u}_{*}^{-1} \partial \widehat{u}_{*}\right) d \bar{z}-\left(\bar{a}_{*}-2 \widehat{u}_{*}^{-1} \partial \widehat{u}_{*}\right) d z\right) \quad \text { and } \quad \widehat{u}_{*} \alpha_{*}
$$

is, respectively, a connection on the product bundle over the $|z| \leq T$ part of $S^{1} \times \mathbb{C}$ and section of this same bundle. 
By construction, the pair in the $\left(A_{n}, \alpha_{n}\right)$ version of (2-11) is gauge equivalent to the pullback of $\left(A_{n}, \alpha_{n}\right)$ via the $r=r_{n}$ version of the rescaling map $\hat{r}_{\gamma}^{-1}$ In particular, given the definition of $(a, \alpha)$ as a limit of the integer $n$ versions of $\left(a_{*}, \alpha_{*}\right)$, a comparison of (2-11) and (2-10) finds that these pairs differ where $|z| \leq T$ by less than $c_{0} \delta$ when $n$ is large.

Let $u_{1 *}$ denote the map from the $|z| \leq T$ part of $S^{1} \times \mathbb{C}$ to $U(1)$ that sends the pair in (2-11) to the $\hat{r}_{\gamma}^{-1}$ pullback of $\left(A_{n}, \alpha_{n}\right)$; thus $u_{1 *}\left(\hat{u}_{*} \alpha_{*}\right)$ is the rescaling of $\alpha_{n}$.

Step 6 Recall from [9, Section 2.c] that any given map c: $S^{1} \rightarrow \mathfrak{C}_{m}$ can be lifted as a pair of smooth connection on the product bundle over $S^{1} \times \mathbb{C}$ and section of this bundle. In particular, [9, Section 2.c] describes such a lift for $\mathfrak{c}_{\gamma}$, this written here as

$$
\left(\theta+A_{0} d t+\frac{1}{2}\left(a^{\prime} d \bar{z}-\bar{a}^{\prime} d z\right), \alpha^{\prime}\right)
$$

This understood, there exists a map $u_{1}: S^{1} \times \mathbb{C} \rightarrow C$ that is smooth along the $\mathbb{C}$ factor, Lipschitz along the $S^{1}$ factor, and such that

$$
a^{\prime}=a+2\left(u_{1} \widehat{u}\right)^{-1} \bar{\partial}\left(u_{1} \widehat{u}\right) \text { and } \quad \alpha^{\prime}=u_{1} \widehat{u} \alpha .
$$

Write $u_{1}=e^{x} u_{0}$ where $u_{0}: S^{1} \rightarrow U(1)$ is a smooth map and $x$ is a map from $S^{1} \times \mathbb{C}$ to $i \mathbb{R}$ that is smooth along the $\mathbb{C}$ factor and Lipschitz along the $S^{1}$ factor and obeys $|x| \leq c_{0} T$ where $|z| \leq T$.

By virtue of what is said in Step 2, the pullback via the $r=r_{n}$ rescaling map $\hat{r}_{\gamma}^{-1}$ of the pair $\left(A_{n}-u_{\gamma 0}^{-1} d u_{\gamma 0}, u_{\gamma 0} \alpha_{n}\right)$ to the $|z| \in\left[\frac{1}{8} T, T\right]$ part of $S^{1} \times \mathbb{C}$ can be written as

$$
\left(\theta+A_{0 *} d t+\frac{1}{2}\left(a_{*}^{\prime} d \bar{z}-\bar{a}_{*}^{\prime} d z\right), \alpha_{*}^{\prime}\right),
$$

where components in (2-14) and their analogs in (2-13) differ by very little when $n$ is large. This is to say that

$$
\left|A_{0 *}-A_{0}\right|+\left|a_{*}^{\prime}-a^{\prime}\right|+\left|\alpha_{*}^{\prime}-\alpha^{\prime}\right| \leq c_{0} \delta
$$

when $n$ is large.

Step 7 Reintroduce $u_{1 *}$ from Part 5 and write the effect of $u_{1}\left(u_{1 *}\right)^{-1}$ on the $\hat{r}_{\gamma}^{-1}$ rescaling of $\left(A_{n}, \alpha_{n}\right)$ as

$$
\left(\theta+A_{0 * *} d t+\frac{1}{2}\left(a_{* *}^{\prime} d \bar{z}-\bar{a}_{* *}^{\prime} d z\right), \alpha_{* *}^{\prime}\right)
$$


Given the less than $c_{0} \delta$ difference between (2-10) and (2-11), it follows that the difference between the respective components of (2-16) and (2-14) where $|z| \leq T$ obeys

$$
\left|A_{0 * *}-A_{0}\right| \leq c_{0} R \quad \text { and } \quad\left|a_{* *}^{\prime}-a^{\prime}\right|+\left|\alpha_{* *}^{\prime}-\alpha^{\prime}\right| \leq c_{0} \delta
$$

when $n$ is large.

To complete the construction, note that it follows from (2-15) and (2-17) that the map $u_{1}\left(u_{1 *}\right)^{-1}$ where $|z| \in\left[\frac{1}{8} T, T\right]$ can be written as $e^{y}$ where $y$ is an $i \mathbb{R}$ valued function on this part of $S^{1} \times \mathbb{C}$ that obeys $\left|x^{\prime}\right| \leq c_{0} \delta$. Keep this in mind and let $\chi^{T}$ denote the function $z \rightarrow \chi(|z| / T)$ on $\mathbb{C}$. Extend the identity map from the $|z| \in\left[\frac{1}{2} T, T\right]$ part of $S^{1} \times \mathbb{C}$ to $U(1)$ over the whole of the $|z|<T$ part as $e^{\chi^{T y}}$ where $|z| \in\left[\frac{1}{8} T, T\right]$ and as $u_{1}\left(u_{1 *}\right)^{-1}$ over the remainder. Use $u_{* *}$ to denote the resulting map. Rescale the latter by the $r=r_{n}$ version of $\widehat{r}_{\gamma}$ and multiply the result with the extension $u_{\gamma 0}$ of $u_{\gamma}$ that was chosen in Step 2. Denote the result by $u_{\gamma}$. It follows from (2-17) that this map from $U_{\gamma}$ to $U(1)$ is admissible in the sense used by Step 3 were it a smooth map. Note in this regard that the derivative bounds on Step 3's pair $b_{\gamma}^{\prime}$ and $A_{0 \gamma}$ follow directly from (2-6) and (2-7) using Lemma 2.3. A smooth map from $U_{\gamma}$ to $U(1)$ sufficiently close to that just constructed will also be admissible and serve for the purposes at hand.

Part 6 Fix $\delta>0$ but small enough to invoke Lemma 2.6 when $n$ is large. Change the notation now and use $\left(A_{n}, \psi_{n}\right)$ to denote the result of applying Lemma 2.6's gauge transformation to the $n$-th element in the subsequence from the sequence that appears in (2-1). Also, use $\left(b_{0}^{\prime}, \eta_{0}^{\prime}\right)$ now to denote the pair $(b, \eta)$ given by Lemma 2.6. In this new notation, $\left(A_{n}, \psi_{n}\right)=\left(A^{*}+2^{1 / 2} r^{1 / 2} b_{0}^{\prime}, \psi^{*}+\eta_{0}^{\prime}\right)$ where $\left|b_{0}^{\prime}\right|+\left|\eta_{0}^{\prime}\right| \leq c_{0} \delta$.

Choose a vector $\zeta \in \mathcal{B}$ with $L_{1}^{2}$ norm less than $\delta$; use $\mathfrak{J}=\left\{\left(\mathfrak{c}_{\gamma}, \zeta_{\gamma}\right)\right\}_{(\gamma, m) \in \Theta}$ to construct the pair $\left(A_{\mathfrak{J}}, \psi_{\mathfrak{J}}\right)$ as instructed in [9, Section 3.a]. The pair $\left(A_{n}, \psi_{n}\right)$ can be written as $\left(A_{\mathfrak{J}}+2^{1 / 2} r^{1 / 2} b_{\zeta}^{\prime}, \psi_{\mathfrak{J}}+\eta_{\zeta}^{\prime}\right)$ where $\left(b_{\zeta}^{\prime}, \eta_{\zeta}^{\prime}\right)=\left(b_{0}^{\prime}, \eta_{0}^{\prime}\right)-\mathfrak{t}_{\zeta}$ where $\mathfrak{t}_{\zeta}=\left(c_{\zeta}, \varsigma_{\zeta}\right)$ is defined by writing $\left(A_{\mathfrak{J}}, \psi_{\mathfrak{J}}\right)=\left(A^{*}+(2 r)^{1^{\prime} / 2} c_{\zeta}, \psi^{*}+\varsigma_{\zeta}\right)$. Note in particular that $\left|\mathfrak{t}_{\zeta}\right| \leq c_{0} \delta$. Thus $\left|b_{\zeta}^{\prime}\right|+\left|\eta_{\zeta}^{\prime}\right| \leq c_{0} \delta$ also. The next lemma supplies a second, $\zeta$-dependent gauge transformation for $\left(A_{n}, \psi_{n}\right)$.

Lemma 2.7 There exists $\kappa \geq 1$ with the following significance: Take $\delta<\kappa^{-1}$ and $\zeta \in \mathcal{B}$ with $L_{1}^{2}$ norm less than $\delta$ to define $\left(A_{\mathfrak{J}}, \psi_{\mathfrak{J}}\right)$. If $n$ is sufficiently large, there exists an $L_{2}^{2}$ map $s=s_{\zeta}: M \rightarrow i \mathbb{R}$ such that

- $|s| \leq c_{0} \delta$ and $|d s| \leq c_{0} \delta^{1 / 2} r^{1 / 2}$.

- The gauge transform of $\left(A_{n}, \psi_{n}\right)$ by $e^{s}$ can be written as $\left(A_{\mathfrak{J}}+2^{1 / 2} r^{1 / 2} b_{\zeta}\right.$, $\left.\psi_{\mathfrak{J}}+\eta_{\zeta}\right)$ where the pair $\left(b_{\zeta}, \eta_{\zeta}\right)$ is of Sobolev class $L_{1}^{2}$, obeys the bottom equation in [9, (3-5)], and also obeys $\left|b_{\zeta}\right|+\left|\eta_{\zeta}\right| \leq c_{0} \delta^{1 / 2}$. 
Proof of Lemma 2.7 If $s$ is of Sobolev class $L_{2}^{2}$, then $\left(b_{\zeta}, \eta_{\zeta}\right)$ are of Sobolev class $L_{1}^{2}$ automatically. Thus, the issue is that of satisfying both the bottom equation in [9, (3-5)] and the norm bound. The construction and analysis of the desired function $s$ has four steps. To simplify the notation, the proof uses $\left(b^{\prime}, \eta^{\prime}\right)$ in lieu of $\left(b_{\zeta}^{\prime}, \eta_{\zeta}^{\prime}\right)$.

Step 1 For the moment, let $s$ denote any given map from $M$ to $U(1)$. The gauge transform of $\left(A_{n}, \psi_{n}\right)$ by $e^{s}$ is

$$
\begin{aligned}
& \left(A_{n}-d s, e^{s} \psi_{n}\right) \\
& \quad=\left(A_{\mathfrak{J}}+2^{1 / 2} r^{1 / 2}\left(b^{\prime}-2^{-1 / 2} r^{-1 / 2} d s\right), \psi_{\mathfrak{J}}+e^{s} \eta^{\prime}+\left(e^{s}-1\right) \psi_{\mathfrak{J}}\right) .
\end{aligned}
$$

This has the form $\left(A_{\mathfrak{J}}+2^{1 / 2} r^{1 / 2} b, \psi_{\mathfrak{J}}+\eta\right)$ with

$$
b=b^{\prime}-2^{-1 / 2} r^{-1 / 2} d s \text { and } \eta=\eta^{\prime}+\left(e^{s}-1\right)\left(\psi_{\mathfrak{J}}+\eta^{\prime}\right) .
$$

Thus, $(b, \eta)$ obeys the bottom equation in $[9,(3-5)]$ if $s$ obeys the equation

$$
\begin{aligned}
d^{\dagger} d s-r\left(e^{-s}-e^{s}\right)\left|\psi_{\mathfrak{J}}\right|^{2}-r\left(\left(e^{-s}-1\right) \eta^{\prime \dagger} \psi_{\mathfrak{J}}-\left(e^{s}-1\right) \psi_{\mathfrak{J}}^{\dagger} \eta^{\prime}\right) & \\
& -2^{1 / 2} r^{1 / 2} d^{\dagger} b^{\prime}-r\left(\eta^{\prime \dagger} \psi_{\mathfrak{J}}-\psi_{\mathfrak{J}}^{\dagger} \eta^{\prime}\right)=0 .
\end{aligned}
$$

A perturbative approach is used in what follows to find a solution to (2-20). To this end, write $e^{-s}-e^{s}=-2 s+\mathfrak{z}(s)$ so as to write (2-20) as

$$
d^{\dagger} d s+2 r\left|\psi_{\mathfrak{J}_{0}}\right|^{2} s+\mathfrak{R}(s)-\mathfrak{P}_{b}-\mathfrak{P}_{\eta}=0,
$$

where

$$
\begin{gathered}
\text { - } \mathfrak{R}(s)=r \mathfrak{z}(s)\left|\psi_{\mathfrak{J}}\right|^{2}+r\left(e^{s}-e^{-s}\right)\left(\left|\psi_{\mathfrak{J}}\right|^{2}-\left|\psi_{\mathfrak{J} 0}\right|^{2}\right) \\
-r\left(\left(e^{-s}-1\right) \eta^{\prime \dagger} \psi_{\mathfrak{J}}-\left(e^{s}-1\right) \psi_{\mathfrak{J}}^{\dagger} \eta^{\prime}\right) \\
\text { - } \mathfrak{P}_{b}=2^{1 / 2} r^{1 / 2} d^{\dagger} b^{\prime} \text { and } \mathfrak{P}_{\eta}=r\left(\eta^{\prime \dagger} \psi_{\mathfrak{J}}-\psi_{\mathfrak{J}}^{\dagger} \eta^{\prime}\right) .
\end{gathered}
$$

A contraction mapping argument is used here to find a small normed solution to (2-21). To this end, note that the operator $d^{\dagger} d+2 r\left|\psi_{\mathfrak{J}_{0}}\right|^{2}$ acting on $C^{\infty}(M ; i \mathbb{R})$ is invertible. Given $p \in M$, let $x \rightarrow G(x, p)$ denote the corresponding Green's function with pole at the point $p$. Note that this function is symmetric in its two entries, smooth on $M-p$, and strictly positive. Moreover, it has a pole as $x \rightarrow p$ that has the form $G(x, p)=(1 /(4 \pi))(1 / \operatorname{dist}(x, p))+O(1)$. With $G$ understood, solution to (2-21) is a fixed point of a map, $\mathrm{T}(\cdot)$ that sends a function $s$ to

$$
\left.\mathrm{T}(s)\right|_{x}=\int_{M} G(x, \cdot)\left(-\mathfrak{R}(s)+\mathfrak{P}_{b}+\mathfrak{P}_{\eta}\right) .
$$

The map $\mathrm{T}$ will be viewed as a map from the Banach space $L^{\infty}(M ; i \mathbb{R})$ to itself. 
Step 2 This contraction mapping strategy requires some bounds for the integrals of the function $x \rightarrow G(x, p)$, the function $x \rightarrow|d G|(x, p)$, and the norm of the two sided derivative $x \rightarrow|d G \overleftarrow{d}|(x, p)$. The next lemma states the bounds that are needed to obtain a fixed point of T. Keep in mind that $G(\cdot, \cdot)$ is symmetric in its two entries and strictly positive.

Lemma 2.8 There exists $\kappa>0$ such that

- $\int_{M} G(x, \cdot) \leq \kappa r^{-1}$.

- $\int_{M}|d G|(x, \cdot) \leq \kappa r^{-1 / 2}$.

- Fix $\rho>0$. Then

(a) $\int_{\operatorname{dist}(x, \cdot)>\rho}|d G \overleftarrow{d}|(x, \cdot) \leq \kappa\left(1+\left|\ln \left(\rho r^{1 / 2}\right)\right|\right)$

(b) $\int_{\operatorname{dist}(x, \cdot)>\rho}|d G|(x, \cdot) \operatorname{dist}(x, \cdot)^{-1} \leq \kappa\left(1+\left|\ln \left(\rho r^{1 / 2}\right)\right|\right)$

at each $x \in M$.

Proof of Lemma 2.8 Fix $\varepsilon>0$ and let $g(\cdot, p)$ denote the Green's function for the operator $d^{\dagger} d+2 \varepsilon^{2} r$ with pole at $p \in M$. This Green function is strictly positive and it obeys

- $g(x, p) \leq c_{0} \frac{1}{\operatorname{dist}(x, p)} e^{-\varepsilon \sqrt{r} \operatorname{dist}(x, p) / c_{0}}$.

- $|d g|(x, p) \leq c_{0} \frac{1}{\operatorname{dist}(x, p)^{2}}\left(1+\varepsilon r^{1 / 2} \operatorname{dist}(x, p)\right) e^{-\varepsilon \sqrt{r} \operatorname{dist}(x, p) / c_{0}}$.

- $|d g \overleftarrow{d}|(x, p) \leq c_{0} \frac{1}{\operatorname{dist}(x, p)^{3}}\left(1+\varepsilon^{2} r \operatorname{dist}(x, p)^{2}\right) e^{-\varepsilon \sqrt{r} \operatorname{dist}(x, p) / c_{0}}$

In any event, use the Green's function $g$ to write $G$ as

$$
G(x, p)=g(x, p)-\int_{M} G(x, \cdot) r\left(\left|\psi_{\mathfrak{J}_{0}}\right|^{2}-\varepsilon^{2}\right) g(\cdot, p) .
$$

Note also that $G>0$. This is a consequence of the maximum principle.

To obtain the first item in the lemma, introduce for each $(\gamma, m) \in \Theta$ the set $U_{\gamma, \varepsilon} \subset U_{\gamma}$ where $\left|\psi_{J_{0}}\right| \leq \varepsilon$. Given that $G>0$, it follows from (2-25) that

$$
G(x, p) \leq g(x, p)+c_{0} \sum_{(\gamma, m) \in \Theta} r \int_{U_{\gamma, \varepsilon}} G(x, \cdot) g(\cdot, p) .
$$


Use $r^{-1} m$ to denote the maximum value of the function on $M$ whose value at $x$ is the integral over $M$ of $G(x, \cdot)$. Integrating (2-26) with respect to $p$ and using the fact that $G$ is positive gives

$$
m \leq c_{0} \varepsilon^{-2}+m c_{0} \varepsilon^{1 / c_{0}} .
$$

If $\varepsilon<1 / c_{0}$, then this last equation bounds $m$ by an $r$-independent constant. This proves the lemma's top most inequality.

To obtain the lemma's second item, differentiate (2-25) with respect to $p$, and then integrate result with respect to $x$. It follows from (2-24), and the lemma's top most inequality that the result is bounded by $c_{0} r^{-1 / 2}$. This and the fact that $G(\cdot, \cdot)$ is symmetric in its entries implies the lemma's middle inequality.

Part (a) of the lemma's third bullet follows by differentiating (2-25), once with respect to $x$, and once with respect to $p$. Integrate the result with respect to $p$, and then use the lemma's middle inequality with the second and third items in (2-24) to deduce the desired bound. Part (b) of the lemma's third bullet is obtained by first differentiating (2-25) with respect to $x$. Then take the norm of both sides, divide by $\operatorname{dist}(x, p)$ and integrate with respect to $p$. An upper bound by $\kappa\left|\ln \left(\rho r^{1 / 2}\right)\right|$ for the right hand side of the resulting expression follows using the second item of the lemma with the first item in (2-24).

Step 3 The top inequality in Lemma 2.8 implies that the norm of the contribution to $\mathrm{T}$ from the integral of $\left.G(x, \cdot) \Re(s)\right|_{(\cdot)}$ is bounded by $c_{0}|s|\left(|s|+\|\zeta\|_{L_{1}^{2}}+\delta\right)$ if $|s| \leq 1$. Meanwhile, this same top inequality bounds the contribution to $T$ from the integral of $\left.G(x, \cdot) \mathfrak{P}_{\eta}\right|_{(.)}$by $c_{0} \delta$. Integrate by parts and then appeal to the middle inequality of Lemma 2.8 to see that the contribution to T from the integral of $\left.G(x, \cdot) \mathfrak{P}_{b}\right|_{(\cdot)}$ is also bounded by $c_{0} \delta$. Granted such bounds, it then follows that $\mathrm{T}$ maps a ball in $L^{\infty}(M ; i \mathbb{R})$ of radius $c_{0}^{-1}$ to itself if $\delta<c_{0}^{-1}$. A similar analysis proves that $\mathrm{T}$ is a contraction mapping on a radius $c_{0}^{-1}$ ball if $\delta<c_{0}^{-1}$. Thus, $\mathrm{T}$ has a unique fixed point on such a ball, and this fixed point has sup norm bounded by $c_{0} \delta$.

Let $s$ now denote the fixed point of the map $\mathrm{T}$ in this radius $c_{0}^{-1}$ ball. The right hand side of (2-23) is a $C^{1}$ function (for any $s \in L^{\infty}$ ) and as a consequence, the fixed point of the map $s$ is $C^{1}$. Given that $\zeta$ and $\left(b^{\prime}, \eta^{\prime}\right)$ are smooth, the right hand side of (2-23) can be differentiated to see that T's fixed point $s$ is $C^{2}$. Thus, $s$ obeys (2-20). Taking limits of smooth elements shows that $s$ obeys (2-20) when $\zeta$ is not smooth but of Sobolev class $L_{2}^{2}$. This understood, and given the right hand side of (2-20) is in $L^{2}$, it follows that $s$ is an $L_{2}^{2}$ function on $M$ in the general case. Continuing in this "boot strapping" vein will prove that $s$ is smooth. 
Step 4 Given that $|s| \leq c_{0} \delta$, it follows that $\eta$ in (2-29) obeys $|\eta| \leq c_{0} \delta$. The desired bound on $|b|$ by $c_{0} \delta^{1 / 2}$ requires a supremum bound on $|d s|$ by $c_{0} r^{1 / 2} \delta^{1 / 2}$. To obtain such a bound, differentiate the right hand side of (2-23). Having done so, use the second item in Lemma 2.8 to bound the absolute value of the contribution to this derivative from $\mathfrak{R}(s)$ and $\mathfrak{P}_{\eta}$ by $c_{0} r^{1 / 2} \delta$.

Write $b^{\prime}=b_{0}^{\prime}+c_{\zeta}$. To bound the $d^{\dagger} b_{0}^{\prime}$ part of the contribution from $\mathfrak{P}_{b}$ to $|d s|$, fix $\rho \in\left(0, r^{-1 / 2}\right)$, and let $\chi^{x, \rho}(\cdot)$ denote the function on $M$ whose value at $p$ is $\chi(\operatorname{dist}(x, p) / \rho)$. Write the contribution to $d s=d \mathrm{~T}(s)$ at $x$ from $2^{1 / 2} r^{1 / 2} d^{\dagger} b^{\prime}$ as

$$
(2 r)^{1 / 2} \int_{M} d G(x, \cdot) \chi^{x, \rho} d^{\dagger} b_{0}^{\prime}+(2 r)^{1 / 2} \int_{M} d G(x, \cdot)\left(1-\chi^{x, \rho}\right) d^{\dagger} b_{0}^{\prime} .
$$

To bound the leftmost term in (2-28), integrate by parts so as to remove the derivative from $b^{\prime}$. Then use the second item and Part (a) of the third bullet of Lemma 2.8 to see that the result is bounded by $c_{0} \delta r^{1 / 2}\left(\left|\ln \left(\rho r^{1 / 2}\right)\right|+\left(\rho r^{1 / 2}\right)^{-1}\right)$. Use (2-25) and the fact that $\left|\nabla b_{0}^{\prime}\right| \leq c_{0} r^{1 / 2}$ to see that the right most term in (2-28) is bounded by $c_{0} r \rho\left(1+\rho r^{1 / 2}\right)$. Granted all of this, it follows that the integrals in (2-28) are bounded by

$$
c_{0} r^{1 / 2}\left(\delta\left(1+\left|\ln \left(\rho r^{1 / 2}\right)\right|\right)+\delta /\left(\rho r^{1 / 2}\right)+(\rho r)^{1 / 2}\right) .
$$

This understood, take $\rho=\delta^{1 / 2} r^{-1 / 2}$ to bound on the $d^{\dagger} b_{0}^{\prime}$ part of the contribution from $\mathfrak{P}_{b}$ to $|d s|$ by $c_{0} \delta^{1 / 2} r^{1 / 2}$.

A bound on the $d^{\dagger} c_{\xi}$ part of the contribution from $\mathfrak{P}_{b}$ to $|d s|$ is obtained as follows: The contribution can be written as in (2-28) but with $c_{\zeta}$ replacing $b_{0}^{\prime}$. Take $\rho$ in this case to equal $r^{-1 / 2}$. To bound the $c_{\zeta}$ version of the right most integral in (2-28), integrate by parts and use the second item and Part (a) of the third bullet of Lemma 2.8 to bound the result by $c_{0} r^{1 / 2} \delta$. As explained next, the $c_{\zeta}$ version of the leftmost integral in (2-28) is also bounded by $c_{0} r^{1 / 2} \delta$.

The asserted bound on the $c_{\xi}$ version of the leftmost integral in (2-28) exploits the fact that $L_{1}^{2}$ functions on $S^{1}$ are Holder continuous with exponent $1 / 2$. This has the following consequence: If $x$ and $x^{\prime}$ are given points in $M$, then $\left.c_{\xi}\right|_{x}-\left.c_{\xi}\right|_{x^{\prime}}$ has norm bounded by $c_{0} r^{1 / 4} \operatorname{dist}\left(x, x^{\prime}\right)^{1 / 2}\|\zeta\|_{L_{1}^{2}}$ at points where $\left|x-x^{\prime}\right| \leq r^{-1 / 2}$. Keep this fact in mind.

For each $k \in\{0,1,2, \ldots\}$, set $\rho_{k}=2^{-k} r^{-1 / 2}$ and remark that the function $\chi^{x, \rho}$ can be written as $\sum_{n=0,1, \ldots} \prod_{j=0, \ldots, k} \chi^{x, \rho_{j}}\left(1-\chi^{x, \rho_{k+1}}\right)$. Thus, the $c_{\xi}$ version of the leftmost integral in $(2-28)$ is

$$
\sum_{k=0,1, \ldots}(2 r)^{1 / 2} \int_{M} d G(x, \cdot) \prod_{j=0, \ldots, k} \chi^{x, \rho_{j}}\left(1-\chi^{x, \rho_{k+1}}\right) d^{\dagger} c_{\zeta} .
$$


Note that the $k$-th summand in (2-30) is supported where the distance $\operatorname{dist}(x, \cdot) \in$ $\left[2^{-k-r} r^{-1 / 2}, 2^{-k} r^{-1 / 2}\right]$. Note also that any given coordinate derivative of $c_{\zeta}$ on the ball of radius $r^{1 / 2}$ about $x$ is the same as that of $\left(c_{\zeta}-\left.c_{\zeta}\right|_{x}\right)$. This last point facilitates the use of the Holder bound for $c_{\zeta}$.

Granted the preceding, integrate by parts in the $k$-th summand and so bound the latter by the integral of $c_{0}\left(\left.|d G \overleftarrow{d}|(x, \cdot)|+| d G\right|_{(x, \cdot)} \mid \operatorname{dist}(x, \cdot)^{-1}\right)$ times $\left|c_{\zeta}-c_{\xi}\right|_{x}$ over the annulus where $\operatorname{dist}(x, \cdot) \in\left[2^{-k-2} r^{-1 / 2}, 2^{-k} r^{-1 / 2}\right]$. Granted what was said about Holder continuity, it follows using both Parts (a) and (b) of the third bullet of Lemma 2.8 that the latter integral is no greater than $c_{0}(1+k) 2^{-k / 2}\|\zeta\|_{L_{1}^{2} \text {. Since }}$ $\sum_{k=0,1, \ldots}(1+k) 2^{-k / 2}$ is convergent, it follows that (2-30) is bounded by $c_{0} r^{1 / 2} \delta$.

Part 7 Fix $\delta>0$ as in Lemma 2.7 take $n$ very large. Lemma 2.7 assigns to each element $\zeta \in \mathcal{B}$ with $L_{1}^{2}$ norm less than $\delta$ a gauge transformation of $\left(A_{n}, \psi_{n}\right)$ with the following properties: Let $\mathfrak{J}=\left\{\left(\mathfrak{c}_{\gamma}, \zeta_{\gamma}\right)\right\}_{(\gamma, m) \in \Theta}$. The gauge transform of $\left(A_{n}, \psi_{n}\right)$ can be written as $\left(A_{\mathfrak{J}}+2^{1 / 2} r^{1 / 2} b_{\zeta}, \psi_{\mathfrak{J}}+\eta_{\zeta}\right)$ where $\mathfrak{b}_{\zeta}=\left(b_{\zeta}, \eta_{\zeta}\right)$ obeys the third equation in [9, (3-5)] and is such that $\left|b_{\zeta}\right|+\left|\eta_{\zeta}\right| \leq c_{0} \delta^{1 / 2}$. Introduce the Hilbert space $\mathbb{H}$ as described in [9, Step 1 of Section 3.b]. This is the completion of $C^{\infty}\left(M ; i T^{*} M \oplus \mathbb{S} \oplus i \mathbb{R}\right)$ using the norm that is given by replacing the integration domain $\mathbb{R} \times M$ in (1-13) by $M$. Then $\mathfrak{b}_{\zeta} \in \mathbb{H}$ since the gauge transformation supplied by Lemma 2.7 is of Sobolev class $L_{2}^{2}$. Choose $\delta<1 / c_{0}$ so that the projection $\Pi_{\zeta}$ on $\mathbb{H}$ as described in [9, Section 3.b] is defined when $\zeta$ has $L_{1}^{2}$ norm less than $\delta$.

Lemma 2.9 There exists $\kappa>1$ with the following significance: If $\delta<\kappa$ and if $n$ is sufficiently large, then there exists a unique $\zeta$ in $\mathcal{B}$ with $L_{1}^{2}$ norm bounded by $\delta$ and such that $\Pi_{\zeta} \mathfrak{b}_{\zeta}=0$.

This lemma is proved in Part 8.

This lemma leads directly to a contradiction with the assumptions in (2-1) when $n$ is large. To see how this comes about, let $\zeta$ now denote the element in $\mathcal{B}$ given by Lemma 2.9. Then $\mathfrak{b}_{\zeta}$ obeys [9, (3-5)] and also [9, (3-16)]. As it is in $\mathbb{H}_{\zeta} \frac{\perp}{\text {, }}$, it follows using [9, (3-16)] and [9, Lemma 3.2] that the $\mathbb{H}$-norm of $\mathfrak{b}_{\zeta}$ obeys $\left\|\mathfrak{b}_{\zeta}\right\|_{\mathbb{H}} \leq$ $c_{0} r^{1 / 2}\left\|\mathfrak{b}_{\zeta} * \mathfrak{b}_{\zeta}\right\|_{2}+c_{0} r^{-1 / 2}$. Since $\left|\mathfrak{b}_{\zeta}\right| \leq \kappa \delta^{1 / 2}$, this implies that

$$
\left\|\mathfrak{b}_{\zeta}\right\|_{\mathbb{H}} \leq c_{0} \delta^{1 / 2}\left\|\mathfrak{b}_{\zeta}\right\|_{\mathbb{H}}+c_{0} r^{-1 / 2}
$$

As a consequence, $\left\|\mathfrak{b}_{\zeta}\right\|_{\mathbb{H}} \leq c_{0} r^{-1 / 2}$ if $\delta<c_{0}^{-1}$. This small $\mathbb{H}$-norm implies that $\mathfrak{b}_{\zeta}$ is the solution to [9, (3-16)] given in [9, Lemma 3.5]. Suppose that $\delta$ is also less than the constant $\varepsilon_{1}$ in [9, Lemma 3.8]. As [9, (3-5)] is obeyed, so is [9, (3-35)]. Thus, $\zeta$ must be the solution to this last equation given by [9, Lemma 3.8]. As a consequence, 
the equivalence class of $\left(A_{n}, \psi_{n}\right)$ is in the image of the map $\Phi^{r}$, which contradicts what is asserted in (2-1).

Part 8 This part contains the following.

Proof of Lemma 2.9 Let $\Pi$ denote the $\zeta=0$ version of the projection $\Pi_{\zeta}$. The first point to make here is that $\Pi_{\zeta} \mathfrak{b}_{\zeta}=0$ if and only if $\Pi\left(\Pi_{\zeta} \mathfrak{b}_{\zeta}\right)=0$ when $\delta<c_{0}^{-1}$ and $n$ is large. Indeed, this follows readily from what is said in [9, Part 8 of Section 2.a]. Now let $t: \mathcal{K} \rightarrow \mathbb{H}$ denote the $\zeta=0$ version of the map that is defined by [10, (2-5)]. This map $t$ identifies $\mathcal{K}$ with the image of $\Pi$. Thus, $\Pi \mathfrak{b}=0$ if and only if $t^{\dagger}(\mathfrak{b})=0$. Note in this regard the fact, implied by [10, (2-7)], that $t$ is nearly isometric with respect to the $L^{2}$ inner products on $\mathcal{K}$ and $\mathbb{H}$.

These first two points have the following consequence: If $\delta<c_{0}^{-1}$ and $n$ is large, then the condition $\Pi_{\zeta} \mathfrak{b}_{\zeta}=0$ holds if and only if $t^{\dagger}\left(\Pi_{\zeta} \mathfrak{b}_{\zeta}\right)=0$. Meanwhile, the assignment $\zeta \rightarrow t^{\dagger}\left(\Pi_{\zeta} \mathfrak{b}_{\zeta}\right)$ can be viewed as a map from the radius $\delta$ ball in $\mathcal{B}$ to the ambient Hilbert space $\mathcal{K}=\bigoplus_{(\gamma, m) \in \Theta} L_{1}^{2}\left(S^{1}, \mathfrak{c}_{\gamma}^{*} T_{1,0} \mathfrak{C}_{m}\right)$. Write any given $(\gamma, m) \in \Theta$ component of $t^{\dagger}(\cdot)$ as $t^{\dagger}(\cdot)_{\gamma}$. It is argued momentarily that the map

$$
\zeta=\left(\zeta_{\gamma}\right)_{(\gamma, m) \in \Theta} \rightarrow\left(\zeta_{\gamma}+r_{\gamma}^{1 / 2} t^{\dagger}\left(\Pi_{\zeta} \mathfrak{b}_{\zeta}\right)_{\gamma}\right)_{(\gamma, m) \in \Theta}
$$

sends a ball of radius $c_{0}^{-1}$ about the origin in $\mathcal{B}$ to itself as a contraction mapping if $\delta<c_{0}^{-1}$ and $n$ is large. Such being the case, the contraction mapping theorem implies that this map has a unique fixed point; and this fixed point is the desired point $\zeta$ with $\Pi_{\zeta} \mathfrak{b}_{\zeta}=0$.

The six steps that follow explain why the map in (2-32) is a contraction mapping on a ball of radius $c_{0}^{-1}$ if $\delta<c_{0}^{-1}$ and $n$ is large.

Step 1 Fix $\zeta=\left(\zeta_{\gamma}\right)_{(\gamma, m) \in \Theta} \in \mathcal{B}$ with $L_{1}^{2}$ norm less than $\delta$, take $\mathfrak{J}=\left\{\left(\mathfrak{c}_{\gamma}, \zeta_{\gamma}\right)\right\}_{(\gamma, m) \in \Theta}$ and write $\left(A_{\mathfrak{J}}, \psi_{\mathfrak{J}}\right)$ as $\left(A_{\mathfrak{J}_{0}}+\left(2 r^{1 / 2}\right) c_{\zeta}, \psi_{\mathfrak{J}_{0}}+\varsigma_{\zeta}\right)$. Set $\mathfrak{t}_{\zeta}=\left(c_{\zeta}, \varsigma_{\zeta}\right)$. As noted previously, $\left|\mathfrak{t}_{\zeta}\right| \leq c_{0} \delta$. In any event, it follows from [9, (2-11), (2-12)] and the formula in $[10,(2-5)]$ for $t$ that any given $(\gamma, m) \in \Theta$ component of $t^{\dagger}\left(t_{\zeta}\right)$ obeys

$$
r_{\gamma}^{1 / 2} t^{\dagger}\left(\mathfrak{t}_{\zeta}\right)=\zeta_{\gamma}+r(\zeta)_{\gamma}
$$

where the $L_{1}^{2}$ norm of $r(\zeta)_{\gamma}$ is bounded by $c_{0}\left(r^{-1 / 2}+\delta\right)\left\|\zeta_{\gamma}\right\|_{L_{1}^{2}}$. By the same token, if $\zeta$ and $\zeta^{\prime}$ are both vectors in $\mathcal{B}$ with $L_{1}^{2}$ norm bounded by $\delta$, then

$$
\left\|r(\zeta)_{\gamma}-r\left(\zeta^{\prime}\right)_{\gamma}\right\|_{L_{1}^{2}} \leq c_{0}\left(r^{-1 / 2}+\delta\right)\left\|\zeta-\zeta^{\prime}\right\|_{L_{1}^{2}}
$$


Step 2 Define the pair $\mathfrak{b}_{*}=\left(b_{*}, \eta_{*}\right)$ as in [10, Step 2 of Section 2.a]. Use this pair to define the operator $\mathfrak{L}_{*}$; this given here in [10, (1-14)] as (1-9). Introduce $\mathfrak{b}_{0}^{0}$ as in [9, Step 2 of the proof of Lemma 3.10]. Define the function $\chi_{0 *}=1-\sum_{(\gamma, m) \in \Theta} \chi_{\gamma}$ and set $\mathfrak{f}=\mathfrak{b}_{\zeta}+\mathfrak{t}_{\zeta}-\mathfrak{b}_{*}-\chi_{0 *} \mathfrak{b}_{0}^{0}$. This step and the next given bounds for the size of $\mathfrak{f}$. The relevance of $\mathfrak{f}$ to the task at hand is explained in Step 5 .

Given that $\mathfrak{b}_{\zeta}$ obeys

$$
\mathfrak{L}_{*} \mathfrak{b}_{\zeta}+r^{1 / 2}\left(\mathfrak{t}_{\zeta} * \mathfrak{b}_{\zeta}+\left(\mathfrak{b}_{\zeta}-\mathfrak{b}_{*}\right) * \mathfrak{b}_{\zeta}\right)=r^{-1 / 2} \mathfrak{v}_{\zeta},
$$

it follows that $\mathfrak{f}$ obeys

$$
\mathfrak{L}_{*} \mathfrak{f}+r^{1 / 2} \mathfrak{b}_{\zeta} * \mathfrak{f}=r^{-1 / 2} \mathfrak{v}_{\zeta}+\mathfrak{L}_{*} \mathfrak{t}_{\zeta}-\mathfrak{L}_{*}\left(\mathfrak{b}_{*}+\chi_{0 *} \mathfrak{b}_{0}^{0}\right) .
$$

Write $\mathfrak{f}=\mathfrak{f}^{\perp}+t(\theta)$ with $\Pi \mathfrak{f}^{\perp}=0$ as in [10, Step 6 of Section 2.a]. This last equation is used to obtain bounds on the size of $\mathfrak{f}^{\perp}$ and the size of $\theta$.

Keep in mind what is said in Steps 2 and 3 of the proof of [9, Lemma 3.10] about the size of $\mathfrak{b}_{*}$ and $\mathfrak{b}_{0}^{0}$ : The former is bounded by $c_{0} r^{-1 / 2} \sum_{(\gamma, m) \in \Theta} e^{-\sqrt{r} \operatorname{dist}(\cdot, \gamma) / c_{0}}$ and the latter by $c_{0} r^{-1}$. As $\left|\mathfrak{t}_{\zeta}\right| \leq c_{0} \delta$ and $\left|\mathfrak{b}_{\zeta}\right| \leq c_{0} \delta^{1 / 2}$, it follows that $|\mathfrak{f}| \leq c_{0}\left(r^{-1 / 2}+\delta^{1 / 2}\right)$. This and $[10,(2-8)]$ imply that

$$
|t(\theta)| \leq c_{0}\left(r^{-1 / 2}+\delta^{1 / 2}\right) \sum_{(\gamma, m) \in \Theta} e^{-\sqrt{r} \operatorname{dist}(\cdot, \gamma) / c_{0}} .
$$

This $L^{\infty}$ bound on $t(\theta)$ and the $L^{\infty}$ bound on $\mathfrak{f}$ imply that $\left|\mathfrak{f}^{\perp}\right| \leq c_{0} \delta^{1 / 2}$.

Consider first the $(1-\Pi)$ part of $(2-36)$. Write $\mathfrak{b}_{\zeta} * \mathfrak{f}$ in terms of $\mathfrak{f}^{\perp}$ and $t(\theta)$. Given the bound by $c_{0} \delta^{1 / 2}$ on the $L^{\infty}$ norm of $\mathfrak{b}_{\zeta}$ and given (2-37), what is written in the top line of [10, (2-19)] together with [10, (2-20)-(2-21)] imply that

$$
\begin{aligned}
\left\|\mathfrak{f}^{\perp}\right\|_{\mathbb{H}} \leq c_{0} \delta^{1 / 2}\left(r^{1 / 2}\left\|\mathfrak{f}^{\perp}\right\|_{2}+\|t(\theta)\|_{2}\right) \\
\quad+c_{0}\|\theta\|_{L_{1}^{2}}+c_{0}\left\|r^{-1 / 2} \mathfrak{v}_{\zeta}+\mathfrak{L}_{*} \mathfrak{t}_{\zeta}-\mathfrak{L}_{*}\left(\mathfrak{b}_{*}+\chi_{0 *} \mathfrak{b}_{0}^{0}\right)\right\|_{2} .
\end{aligned}
$$

The proof of [9, Lemma 3.10] finds the $L^{2}$ norm of $r^{-1 / 2} \mathfrak{v}_{\zeta}+\mathfrak{L}_{*} \mathfrak{t}_{\zeta}-\mathfrak{L}_{*}\left(\mathfrak{b}_{*}+\chi_{0 *} \mathfrak{b}_{0}^{0}\right)$ is bounded by $c_{0}\left(r^{-1}+r^{-1 / 2}\|\zeta\|_{L_{1}^{2}}\right)$. Meanwhile, from [9, (3-13), (3-24)] with (1-7) and [9, (2-11), (2-12)], the $L^{2}$ norm of $\mathfrak{L}_{*} \mathfrak{t}_{\zeta}$ is bounded by $c_{0}\left(r^{-1 / 2}+\|\zeta\|_{L_{1}^{2}}\right)\|\zeta\|_{L_{1}^{2}}$. Thus, (2-38) implies that

$$
\left\|\mathfrak{f}^{\perp}\right\|_{\mathbb{H}} \leq c_{0}\left(\|\theta\|_{L_{1}^{2}}+\|\zeta\|_{L_{1}^{2}}^{2}+r^{-1}\right)
$$

Step 3 This step considers the image of (2-35) via the adjoint, $t^{\dagger}$, of the map $t$ so as to obtain an $L_{1}^{2}$ bound on $\theta$. It follows from [10, Lemma 2.1] that this has the form

$$
\mathrm{D} \theta+r^{1 / 2} t^{\dagger}\left(\mathfrak{b}_{\zeta} * \mathfrak{f}\right)+\mathfrak{p}^{\dagger}\left(\mathfrak{f}^{\perp}\right)=t^{\dagger}\left(r^{-1 / 2} \mathfrak{v}_{\zeta}+\mathfrak{L}_{*} \mathfrak{t}_{\zeta}-\mathfrak{L}_{*}\left(\mathfrak{b}_{*}+\chi_{0 *} \mathfrak{b}_{0}^{0}\right)\right) .
$$


Given [9, (3-37)] and given the $L^{\infty}$ norm bounds on $\mathfrak{f}$ and $\mathfrak{t}_{\zeta}$, this equality implies

$$
\begin{aligned}
\|\mathrm{D} \theta\|_{L^{2}} \leq c_{0}(\delta & \left.+r^{-1 / 2}\right)\left(\|\theta\|_{L_{1}^{2}}+\|\zeta\|_{L_{1}^{2}}^{2}+r^{-1}\right) \\
& +c_{0}\left\|t^{\dagger}\left(r^{-1 / 2} \mathfrak{v}_{\zeta}+\mathfrak{L}_{*} \mathfrak{t}_{\zeta}\right)\right\|_{L^{2}}+\left\|t^{\dagger}\left(\mathfrak{L}_{*}\left(\mathfrak{b}_{*}+\chi_{0 *} \mathfrak{b}_{0}^{0}\right)\right)\right\|_{L^{2}}
\end{aligned}
$$

To make something of this last inequality, it is necessary to say something about the size of the right most two terms in (2-41). In this regard, $L^{2}$ norm of the far right term is bounded by $c_{0} r^{-1}$ This follows from [9, (3-41), (3-13), (3-14)].

To bound the $L^{2}$ norm of $t^{\dagger}\left(r^{-1 / 2} \mathfrak{v}_{\zeta}+\mathfrak{L}_{*} \mathfrak{t}_{\zeta}\right)$, first fix $(\gamma, m) \in \Theta$ and use [10, (2-19), (2-22)] to identify

$$
r_{\gamma}^{1 / 2} t^{\dagger}\left(\mathfrak{L}_{*} \mathfrak{t}_{\zeta}\right)_{\gamma}=(\mathrm{D} \zeta)_{\gamma}+\mathfrak{e}
$$

where $\|\mathfrak{e}\|_{L^{2}} \leq c_{0}\left(r^{-1 / 2}+\|\zeta\|_{L_{1}^{2}}\right)\left\|\zeta_{\gamma}\right\|_{L_{1}^{2}}$. Then use [10, Lemma 2.1] to write

$$
\left(\mathrm{D} \zeta_{\gamma}\right)=\frac{i}{2} \nabla_{t} \zeta_{\gamma}+\left.\left(\nabla_{\zeta_{\gamma}} \nabla^{1,0} \hbar\right)\right|_{\mathfrak{c}_{\gamma}}+\mathfrak{e}^{\prime}
$$

where $\left\|\mathfrak{e}^{\prime}\right\|_{L^{2}} \leq c_{0} r^{-1 / 2}\left\|\zeta_{\gamma}\right\|_{L_{1}^{2}}$. Meanwhile, use [9, (3-38)] to write

$$
r_{\gamma}^{1 / 2} t^{\dagger}\left(r^{-1 / 2} \mathfrak{v}_{\zeta}\right)_{\gamma}=-\left(\frac{i}{2} \nabla_{t} \zeta_{\gamma}+\left.\left(\nabla_{\zeta_{\gamma}} \nabla^{1,0} h\right)\right|_{\mathfrak{c}_{\gamma}}\right)+\mathfrak{e}^{\prime \prime}
$$

where $\left\|\mathfrak{e}^{\prime \prime}\right\|_{L^{2}} \leq c_{0}\left(r^{-1 / 2}+\|\zeta\|_{L_{1}^{2}}^{2}\right)$. Add (2-43) to (2-44) to obtain a bound on the $L^{2}$ norm of $t^{\dagger}\left(r^{-1 / 2} \mathfrak{v}_{\zeta}+\mathfrak{L}_{*} \mathfrak{t}_{\zeta}\right)_{\gamma}$ by $c_{0}\left(r^{-1}+r^{-1 / 2}\|\zeta\|_{L_{1}^{2}}^{2}\right)$.

Put this last bound into (2-41) and then use [10, Lemma 2.1] to see that

$$
\|\theta\|_{L_{1}^{2}} \leq c_{0}\left(r^{-1}+\delta\|\zeta\|_{L_{1}^{2}}^{2}\right)
$$

Step 4 Fix a pair $\zeta$ and $\zeta^{\prime} \in \mathcal{B}$ and any given $\mathfrak{b} \in \mathbb{H}$ with $|\mathfrak{b}|$ bounded on $M$. This step considers the difference between $t^{\dagger}\left(\Pi_{\zeta^{b}} \mathfrak{b}\right)$ and $t^{\dagger}\left(\Pi_{\zeta^{\prime}} \mathfrak{b}\right)$. It follows from [9, (2-11), (2-12)] that this difference obeys

$$
\left|t^{\dagger}\left(\Pi_{\zeta} \mathfrak{b}\right)_{\gamma}-t^{\dagger}\left(\Pi_{\zeta^{\prime}} \mathfrak{b}_{\gamma}\right)\right| \leq c_{0} r^{-1 / 2}\left|\zeta_{\gamma}-\zeta_{\gamma}^{\prime}\right|\|\mathfrak{b}\|_{L^{\infty}}
$$

$$
\begin{aligned}
& \left\|t^{\dagger}\left(\Pi_{\zeta} \mathfrak{b}\right)_{\gamma}-t^{\dagger}\left(\Pi_{\zeta^{\prime}} \mathfrak{b}\right)_{\gamma}\right\|_{L_{1}^{2}} \\
& \leq c_{0}\left\|\zeta_{\gamma}-\zeta_{\gamma}^{\prime}\right\|_{L_{1}^{2}}\left(r^{-1 / 2}\|\mathfrak{b}\|_{L^{\infty}}+\left(\int_{S^{1} \times \mathbb{C}} \chi\left(|z| / \rho_{*}\right)\left|\nabla_{t} \mathfrak{b}\right|^{2} e^{-\sqrt{r}|z| / c_{0}}\right)^{1 / 2}\right)
\end{aligned}
$$

where $\nabla_{t}$ is the covariant derivative along the $S^{1}$ factor of $S^{1} \times \mathbb{C}$ that is defined using the connection $A^{*}$. 
Step 5 Write $\mathfrak{f}$ as $\mathfrak{f}^{\perp}+t(\theta)$ as done in Steps 2 and 3. Fix a pair $(\gamma, m) \in \Theta$. Then write

$$
\begin{aligned}
& r_{\gamma}^{1 / 2} t^{\dagger}\left(\Pi_{\zeta} \mathfrak{b}_{\zeta}\right)_{\gamma} \\
& \quad=r_{\gamma}^{1 / 2} t^{\dagger}\left(\Pi_{\zeta} \mathfrak{f}\right)_{\gamma}-r_{\gamma}^{1 / 2} t^{\dagger}\left(\Pi_{\zeta} \mathfrak{t}_{\zeta}\right)_{\gamma}+r_{\gamma}^{1 / 2} t^{\dagger}\left(\Pi_{\zeta}\left(\mathfrak{b}_{*}+\chi_{0 *} \mathfrak{b}_{0}^{0}\right)\right)_{\gamma}
\end{aligned}
$$

Now write $\mathfrak{f}=\mathfrak{f}^{\perp}+t(\theta)$ as in Steps 2 and 3. Then (2-48) gives

$$
r_{\gamma}^{1 / 2} t^{\dagger}\left(\Pi_{\zeta} \mathfrak{b}_{\zeta}\right)_{\gamma}=r_{\gamma}^{1 / 2} t^{\dagger}(t(\theta))_{\gamma}-\zeta_{\gamma}+\mathfrak{e}+\mathfrak{e}^{\prime}
$$

where $\mathfrak{e}$ accounts for the difference between $\Pi_{\zeta}$ and $\Pi$, and where

$$
\left\|\mathfrak{e}^{\prime}\right\|_{L_{1}^{2}} \leq c_{0}\left(r^{-1 / 2}+\|\zeta\|_{L_{1}^{2}}^{2}\right) \text {. }
$$

With regards to $\mathfrak{e}$, it follows from (2-39), (2-45) and (2-47) that

$$
\|\mathfrak{e}\|_{L_{1}^{2}} \leq c_{0}\|\zeta\|_{L_{1}^{2}}\left(\delta+r^{1 / 2}\|\zeta\|_{L_{1}^{2}}^{2}+r^{-1 / 2}\right) \text {. }
$$

Given [10, (2-7)] and (2-49), what is written in (2-33), (2-50) and (2-51) implies that the $L_{1}^{2}$ norm of the map in (2-32) is bounded by

$$
c_{0}\|\zeta\|_{L_{1}^{2}}\left(\delta+r^{1 / 2}\|\zeta\|_{L_{1}^{2}}\right)+c_{0} r^{-1 / 2} .
$$

As a consequence, the map in (2-32) sends the ball of radius $c_{0}^{-1} r^{-1 / 4}$ in $\mathcal{B}$ to itself if $\delta<c_{0}^{-1}$ and $r \geq c_{0}$. Granted this, let $\mathcal{B}_{*}$ denote such a ball. Write its radius as $\varepsilon r^{-1 / 4}$.

Step 6 What follows explains how to prove the assertion that (2-32) is a contraction on $\mathcal{B}_{*}$ when $\varepsilon, \delta<c_{0}^{-1}$ and $r \geq c_{0}$. To start, fix a pair $\zeta, \zeta^{\prime} \in \mathcal{B}_{*}$. Define $\mathfrak{f}$ as in Step 2 using $\zeta$ and define $\mathfrak{f}^{\prime}$ to be the $\zeta^{\prime}$ analog. Note that $\mathfrak{u}=\mathfrak{f}-\mathfrak{f}^{\prime}=\mathfrak{b}_{\zeta}-\mathfrak{b}_{\zeta^{\prime}}+\mathfrak{t}_{\zeta}-\mathfrak{t}_{\zeta^{\prime}}$. Subtract the $\mathfrak{f}$ and $\mathfrak{f}^{\prime}$ versions of (2-36) and write what results as

$$
\mathfrak{L}_{*} \mathfrak{u}+r^{1 / 2}\left(\mathfrak{b}_{\zeta^{\prime}}+\mathfrak{f}\right) * u=r^{1 / 2}\left(\mathfrak{t}_{\zeta}-\mathfrak{t}_{\zeta^{\prime}}\right) * \mathfrak{f}+r^{-1 / 2}\left(\mathfrak{v}_{\zeta}-\mathfrak{v}_{\zeta^{\prime}}\right)+\mathfrak{L}_{*}\left(\mathfrak{t}_{\zeta}-\mathfrak{t}_{\zeta^{\prime}}\right)
$$

Now write $\mathfrak{u}=\mathfrak{u}^{\perp}+t(\Delta)$ and mimic what was done in Steps 2 and 3 to bound the $H$ norm of $\mathfrak{u}^{\perp}$ and the $L_{1}^{2}$ norm of $\Delta$. What with (2-36), (2-37) and (2-47), the manipulations done in Step 5 can be repeated with only notational changes to establish the contraction property when $\varepsilon, \delta<c_{0}^{-1}$ and $r \geq c_{0}$. The details of all of this are straightforward and omitted.

\section{2.b Proof of Proposition 2.1}

Fix a pair of functions $(\nu, \mu)$ on $S^{1}$ with $v$ real and $\mu$ having values in $\mathbb{C}$. Use the pair $(\nu, \mu)$ to define the function $f$ on the vortex moduli spaces $\left\{\mathfrak{C}_{m}\right\}_{m \geq 1}$. The upcoming 
Proposition 2.11 describes a compactness theorem for solutions to the corresponding versions of (1-6) and for some related equations. Proposition 2.1 is a special case of Proposition 2.11.

To set the stage for this proposition, suppose that $g: S^{1} \times \mathbb{C} \rightarrow \mathbb{R}$ is a smooth function whose norm grows slower as $|z| \rightarrow \infty$ on $\mathbb{C}$ than some power of $|z|$. The given function $g$ is used to define the smooth function $g: S^{1} \times \mathfrak{C}_{n} \rightarrow \mathbb{R}$ as follows: If $t \in S^{1}$ and $\mathfrak{c}=(A, \alpha)$, set

$$
g(t, \mathfrak{c})=\frac{1}{2 \pi} \int_{\mathbb{C}} g(t, \cdot)\left(1-|\alpha|^{2}\right)
$$

A function of this sort gives a perturbed version of (1-6), this the equation

$$
\frac{i}{2} \mathfrak{c}^{\prime}+\left.\nabla^{(1,0)}(\mathfrak{h}+g)\right|_{\mathfrak{c}}=0 .
$$

The statement of the next proposition uses $\nabla^{\mathbb{C}}$ to denote the vector of partial derivatives on $\mathbb{C}$.

Proposition 2.10 Fix a positive integer, $n$. If $n=1$, assume that $(\nu, \mu)$ is nondegenerate, and if $n>1$, assume that $(\nu, \mu)$ is either hyperbolic or $n$-elliptic. Given $K \geq 1$, there exist $\varepsilon>0$ and $T \geq 1$ with the following significance: Suppose that $g: S^{1} \times \mathbb{C} \rightarrow \mathbb{R}$ is a smooth function with

$$
\sup _{z \in C}\left((K+|z|)^{-2}|g|+(K+|z|)^{-1}\left|\nabla^{\mathbb{C}} g\right|+K^{-1}\left|\nabla^{\mathbb{C}} \nabla^{\mathbb{C}} g\right|\right)<\varepsilon .
$$

Suppose that $\mathrm{c}: S^{1} \rightarrow \mathfrak{C}_{n}$ is a solution to the version of (2-55) that is defined using the function $g$ in (2-54) and the function $f$ in (1-5). Write $\mathfrak{c}=(A, \alpha)$. Then all points in $\alpha^{-1}(0)$ have distance $T$ or less from the origin in $\mathbb{C}$.

The set of maps $\mathfrak{c}: S^{1} \rightarrow \mathfrak{C}_{n}$ that obey (2-55) is given the $C^{\infty}$ Frechet topology via its inclusion in the space $C^{\infty}\left(S^{1} ; \mathfrak{C}_{n}\right)$. The following proposition gives a condition that guarantees the compactness of this space.

Proposition 2.11 Let $n,(v, \mu)$ and $g$ be as described in Proposition 2.10. Define $g$ as in (2-54) and $h$ as in (1-5). Then the space of solutions of the resulting version of (2-55) is compact.

Proof of Proposition 2.11 Given Proposition 2.10, it then follows from what is said in [9, Part 7 of Section 2.a] that there is a compact subset of $\mathfrak{C}_{n}$ that contains the images of all maps from $S^{1}$ to $\mathfrak{C}_{n}$ that obey (2-55). As a consequence of (2-55), the derivatives of all such maps are uniformly bounded. This understood, differentiate (2-55) to see 
that the second derivatives of all such maps are uniformly bounded. Differentiate again and continue in this vein to see that the derivatives to any given order are uniformly bounded. Now apply the Arzela-Ascoli theorem to see that any sequence of maps from $S^{1}$ to $\mathfrak{C}_{n}$ that all solve (2-55) has a subsequence that converges in any given $k \geq 1$ version of the $C^{k}$ topology on the space of maps from $S^{1}$ to $\mathfrak{C}_{m}$. Note that convergence in the $C^{1}$ topology guarantees that the limit map obeys (2-55).

The remainder of this subsection contains the following.

Proof of Proposition 2.10 The proof is broken into seven steps.

Step 1 This step states and then proves a lemma about the clustering of $n$ points in $\mathbb{C}$. Here is the lemma.

Lemma 2.12 Fix a positive integer, $n$, and fix $\rho>1$. Let $\mathfrak{Z}=\left\{z_{1}, \ldots, z_{n}\right\} \in \operatorname{Sym}^{n}(\mathbb{C})$. Then there exists $R \in\left(\rho, \rho^{2^{n}}\right]$ and a partition of $\mathfrak{Z}$ into nonempty subsets that have the following property: The diameter of each partition subset is less than $R$ and the distance between any pair of distinct partition subsets is greater than $R^{2}$.

Proof of Lemma 2.12 Define an equivalence relation on $\mathfrak{Z}$ as follows: Points $z$ and $z^{\prime}$ from $\mathfrak{Z}$ are equivalent if $\left|z-z^{\prime}\right| \leq \rho$. Let $\mathfrak{Z}=\mathfrak{Z}_{11} \cup \cdots \cup \mathfrak{Z}_{1 p}$ denote the resulting partition. If the distance between any two of distinct partition subsets from this partition is greater than $\rho^{2}$, then there exists $R$ slightly greater than $\rho$ that makes the lemma true. If two distinct partition subsets have distance less than or equal to $\rho^{2}$, then define an equivalence relation on the set $\left\{\mathfrak{Z}_{11}, \ldots, \mathfrak{Z}_{1 p}\right\}$ as follows: Any two partition subsets are deemed to be equivalent if their distance is less than or equal to $\rho^{2}$. The resulting equivalence classes define a new partition of $\mathfrak{Z}$, this written as $\mathfrak{Z}_{21} \cup \cdots \cup \mathfrak{Z}_{2 p^{\prime}}$. Note that $p^{\prime}<p$. If the distance between any two distinct partition subsets from this new partition is greater than $\rho^{4}$, then stop because there is a choice for $R \sim \rho^{2}$ that makes the lemma true. Otherwise, rerun this repartitioning exercise using the partition $\left\{\mathfrak{Z}_{2 j}\right\}$ in lieu of $\left\{Z_{1 j}\right\}$ and using $\rho^{2}$ in lieu of $\rho$. Continuing in this vein finds a partition and value of $R \sim \rho^{q}$ for $1 \leq q \leq 2^{n}$ that makes the lemma true because the number partition subsets decreases at each iteration of this step.

Step 2 Fix $p \gg 1$ and let $t \rightarrow \mathfrak{c}(t)$ denote a solution to (2-55). Let $t \rightarrow \mathfrak{Z}(t)$ denote the corresponding map to $\operatorname{Sym}^{n}(\mathbb{C})$ given by writing $\mathfrak{c}(t)=(A, \alpha)$ and taking the zeros of $\alpha$. Fix $t_{0}$ and let $\mathfrak{Z}\left(t_{0}\right)=\mathfrak{Z}_{1} \cup \cdots \cup \mathfrak{Z}_{p}$ denote a partition that is supplied by Lemma 2.12. Thus, there exists $R \sim \rho^{q}$ for $q \in\left\{1, \ldots, 2^{n}\right\}$ such that the diameter of each partition subset is less than $R$ and the separation between any two distinct 
partition subsets is greater than $R^{2}$. Since the corresponding map $t \rightarrow \mathfrak{Z}(t) \in \operatorname{Sym}^{n}(\mathbb{C})$ is continuous, there exists an open neighborhood of $t_{0}$ with the property that if $t^{\prime}$ is in this neighborhood, then $\mathfrak{Z}\left(t^{\prime}\right)$ also has a partition into $p$ sets, each with diameter less than $\frac{5}{4} R$ and with pairwise separation greater than $\frac{3}{4} R^{2}$. These sets are in $1-1$ correspondence with those defined by $t_{0}$ and they vary continuously with $t$ when viewed as maps into the relevant version of $\operatorname{Sym}^{(\cdot)}(\mathbb{C})$. Let $I$ denote such an open neighborhood of $t_{0}$. Write $\mathfrak{Z}(t)=\mathfrak{Z}_{1}(t) \cup \cdots \cup \mathfrak{Z}_{p}(t)$ as $t$ varies $I$.

Step 3 This step derives a lower bound for the length of $I$ in terms of $R$. The following lemma summarizes the story.

Lemma 2.13 Fix $(\nu, \mu)$ as described in Proposition 2.10. Given $K \geq 1$ and $\varepsilon_{0}>0$, there exists a constant, $\kappa>1$, with the following significance: Suppose that $g: S^{1} \rightarrow \mathbb{C}$ is a smooth function that obeys the bound given by Proposition 2.10 with $\varepsilon<\varepsilon_{0}$. Let $\mathfrak{c}$ denote a solution to (2-55) as defined using $(\nu, \mu)$ and the function $h$ given by (1-5) and $g$ by (2-54). Let $\mathfrak{Z}$ denote the corresponding map from $S^{1}$ to $\mathfrak{C}_{n}$. Suppose that $R>\kappa$ is such that $\mathfrak{Z}\left(t_{0}\right)$ has a partition whereby each partition subset has diameter less than $R$, and whereby the distance between any two distinct partition subsets is greater than $R^{2}$. Then, an interval $I$ can be chosen as in Step 2 above so as to contain $\left[t_{0}-\kappa^{-1} R^{-2}, t_{0}+\kappa^{-1} R^{-2}\right]$.

Proof of Lemma 2.13 Take $I$ to be the largest interval that contains $t_{0}$ and has the desired properties. If $t^{\prime} \in \partial I$, then one or both of the following must occur:

- There exists $j \in\{1, \ldots, p\}$ such that $\mathfrak{Z}_{j}\left(t^{\prime}\right)$ has diameter $\frac{5}{4} R$.

- There exists $i \neq j \in\{1, \ldots, p\}$ such that $\operatorname{dist}\left(\mathfrak{Z}_{i}\left(t^{\prime}\right), \mathfrak{Z}_{j}\left(t^{\prime}\right)\right)=\frac{3}{4} R^{2}$.

As is explained next, it takes time $O\left(R^{-1}\right)$ for the first instance to occur. This can be argued as follows: For each $j$, use $\mathfrak{c}_{j}^{t}(t)=\left(A_{j}, \alpha_{j}\right)$ to denote the solution to (1-4) that is defined by $\mathfrak{Z}_{j}(t)$. Thus, $\mathfrak{c}_{j}^{t}(t)=\left(A_{j}^{t}, \alpha_{j}^{t}\right) \in \operatorname{Sym}^{m}(\mathbb{C})$ with $m=m_{j}$ depending on $j$. Each $\mathfrak{c}_{j}^{t}(\cdot)$ defines a smooth map from $I$ to the $m=m_{j}$ version of $\mathfrak{C}_{m}$. Introduce $z_{j}(t)$ to denote the center of $\mathfrak{Z}_{j}(t)$; this given by $\left(1 / m_{j}\right) \sum_{z \in \mathfrak{Z}_{j}(t)} z$. Define $\mathfrak{c}_{j}(t) \in \mathfrak{C}_{m_{j}}$ so that its translate by $z_{j}(t)$ is $\mathfrak{c}_{i}^{t}$.

With the preceding understood, now write $\mathfrak{c}(t)=(A, \alpha)$. It then follows from [9, (2-4)] that $\alpha=e^{u} \prod_{j} \alpha_{j}^{t}$ where $|\operatorname{re}(u)| \leq c_{0} \sum_{i \neq j} e^{-\sqrt{2}\left|z_{i}-z_{j}\right|}$. Moreover, it follows from $[9,(2-4),(2-5)]$ that the function $h+g$ that appears in (2-55) can be written as

$$
\mathfrak{h}+g=\frac{1}{2} \sum_{j} m_{j}\left(2 v\left|z_{j}\right|^{2}+\mu \bar{z}_{j}^{2}+\bar{\mu} z_{j}^{2}+g\left(z_{j}\right)\right)+\sum_{j} g_{j}\left(c_{j}\right)+\mathfrak{r},
$$


where $g_{j}$ is defined as in (2-54) using a function, $g_{j}$ that obeys $\left|g_{j}\right| \leq c_{K}|z|$. Meanwhile, $|\mathfrak{r}|+|d \mathfrak{r}| \leq c_{K} \sum_{i \neq j} e^{-\sqrt{2}\left|z_{i}-z_{j}\right|}$. Here, and in what follows, $c_{K}$ depends only on the constant $K$ and $(v, \mu)$. The precise value of this constant can increase with each appearance.

This last point with [9, (2-5), (2-16)] imply that

$$
\left|\frac{d}{d t} \mathfrak{c}_{j}(t)\right| \leq c_{K} R
$$

This inequality implies that the first item in (2-56) can arise only if $\left|t^{\prime}-t_{0}\right| \leq c_{K} R^{-1}$.

Turn now to the second item in (2-56). It follows from [9, (2-16) and (2-3)] with (2-57) that the point path $t \rightarrow z_{j}(t)$ moves while $t \in I$ in accord with an equation that has the schematic form

$$
\frac{i}{2} \frac{d}{d t} z_{j}+v z_{j}+\mu \bar{z}_{j}=\mathfrak{r}_{j},
$$

where $\left|\mathfrak{r}_{j}\right|<\varepsilon\left|z_{j}\right|+c_{K}\left(1+\sum_{i: i \neq j} e^{-\sqrt{2}\left|z_{i}-z_{j}\right|}\right)$. This last observation has the following consequence: If $\frac{1}{2} R^{2} \leq\left|z_{i}-z_{j}\right| \leq \frac{3}{2} R^{2}$, then

$$
\left|\frac{d}{d t}\left(z_{i}-z_{j}\right)\right| \leq c_{K} R^{2}
$$

This implies that the second item in (2-56) can occur only if $\left|t^{\prime}-t_{0}\right| \leq c_{K} R^{-2}$.

Step 4 Suppose now that Proposition 2.10 is false. If this is the case, there exist $K \geq 1$ plus, for each $v \in\{1,2, \ldots\}$, the following:

- A positive number $\varepsilon_{v}$.

- A function $g=g_{v}$ on $S^{1} \times \mathbb{C}$ that obeys the assumptions in Proposition 2.10 with constant $\varepsilon=\varepsilon_{v}$.

- A map $\mathfrak{c}^{v}: S^{1} \rightarrow \mathfrak{C}_{n}$ that solves the version of (2-55) that is defined using $(\nu, \mu)$ to define $h$ and $g_{v}$ to define $g$.

And, this data has two properties. Here is the first: The sequence $\left\{\varepsilon_{v}\right\}$ is decreasing with limit zero. To state the second, let $\mathfrak{Z}^{v}(\cdot)$ denote the map from $S^{1}$ to $\operatorname{Sym}^{n}(\mathbb{C})$ that corresponds to $\mathfrak{c}^{v}$. Let $d_{v}=\sup _{t \in S^{1}} \operatorname{dist}\left(\left.\mathfrak{Z}^{v}\right|_{t}, 0\right)$. Here, 0 denotes the point $(0, \ldots, 0) \in \operatorname{Sym}^{n}(\mathbb{C})$. Granted this terminology, here is the second property: The sequence $\left\{d_{v}\right\}$ is increasing and unbounded. 
What follows is a consequence of Lemma 2.13. There exists

- A positive integer $N$, a constant $c_{0}>0$, and a cover of $S^{1}$ by $N$ or less intervals of length at least $c_{0} R^{-2}$.

- There exists a subsequence of $\left\{c^{v}\right\}$ such that on each interval of the cover and for each index $v$ in the subsequence, the corresponding $\mathfrak{Z}^{v}(\cdot) \in$ $\operatorname{Sym}^{n}(\mathbb{C})$ has a partition as $\mathfrak{Z}^{v}=\left\{\mathfrak{Z}_{1}^{v}, \ldots, \mathfrak{Z}_{p}^{v}\right\}$ where

(a) $\mathfrak{Z}_{j}^{v}(\cdot) \in \operatorname{Sym}^{m_{j}}(C)$ has diameter less than $\frac{5}{4} R$.

(b) $\operatorname{dist}\left(\mathfrak{Z}_{i}^{v}, \mathfrak{Z}_{j}^{v}\right)>\frac{3}{4} R^{2}$ when $i \neq j$.

(c) The integers $p$ and $\left\{m_{j}\right\}_{1 \leq j \leq p}$ depend on $I$ but not on the index $v$.

Pass to the subsequence that is described by (2-62) and renumber the latter starting from one.

Step 5 Fix an index $v$ and an interval from the cover given in (2-62). Use $\mathfrak{c}^{v}$ in lieu of $\mathfrak{c}$ to define the collection of functions $\left\{z_{j}^{v}\right\}_{1 \leq j \leq p}$ on this interval. For each $j$, set $w_{j}^{v}=d_{v}^{-1} z_{j}^{v}$. By virtue of (2-61), the collection $\left\{w_{j}^{v}\right\}$ obeys the equation

$$
\frac{i}{2} \frac{d}{d t} w_{j}^{v}+v w_{j}^{v}+\mu \bar{w}_{j}^{v}=d_{v}^{-1} \mathfrak{r}_{j}^{v} .
$$

One consequence of this is that the collection $\left\{w_{j}^{v}\right\}$ vary on their interval of definition with first derivatives that are bounded by an index $v$ independent constant.

For each $t$ on the interval where $\left\{w_{j}^{v}\right\}$ is defined, set

$$
\wp_{v}(t ; z)=\prod_{1 \leq j \leq p}\left(z-w_{j}^{v}(t)\right)^{m_{j}} .
$$

The derivatives of the coefficients of $\wp_{v}(t, z)$ are uniformly bounded on their interval of definition. Moreover, if $\wp_{v}(\cdot, z)$ and $\wp_{v}^{\prime}(\cdot, z)$ come from intervals of the cover that overlap, then their coefficients for any given power of $z$ differ by no more than $c_{0} R / d_{v}$.

Step 6 It follows from what is said in the previous step that there is a subsequence of the index set with the property that the coefficients of the corresponding sequence of polynomials $\left\{\wp_{v}(\cdot, z)\right\}$ converge to give continuous and piecewise differentiable functions on $S^{1}$. Let $\wp(\cdot, z)$ denote the resulting degree $n$ polynomial. It follows now from the definition of $\left\{w_{j}^{v}\right\}$ that the roots of $\wp(t, \cdot)$ lie in the closed unit disk for each $t \in S^{1}$, and that there exists a nonempty, open set in $S^{1}$ where $\wp(t, \cdot)$ has at least one nonzero root. Let $p(\cdot)$ denote the function on $S^{1}$ whose value at $t$ is the number of distinct, nonzero roots of $\wp(t, \cdot)$. Fix $t_{0} \in S^{1}$ where $p(\cdot)$ has its maximum. Then $p(\cdot)=p\left(t_{0}\right)$ on some neighborhood of $t_{0}$. Let $I \subset S^{1}$ denote the maximal open 
interval in $S^{1}$ that is symmetric about $t_{0}$ and has this property. Write this interval as $\left(t_{0}-a, t_{0}+a\right)$ with $a \in(0, \pi]$. When $t \in I$, use $\left\{w_{1}(t), \ldots, w_{p\left(t_{0}\right)}(t)\right\}$ to denote the distinct, nonzero roots of $\wp(t, \cdot)$. Each is a differentiable functions of $t$. Moreover, by virtue of (2-61), each obeys

$$
\frac{i}{2} \frac{d}{d t} w_{j}+v w_{j}+\mu \bar{w}_{j}=0
$$

Step 7 Standard uniqueness properties for first order, linear equations of the sort that appear in (2-65) guarantee that none of the collection $\left\{w_{j}\right\}$ can vanish at $t_{0} \pm a$. Likewise, $w_{i}=w_{j}$ at $t_{0} \pm a$ only if $i=j$. This requires that $a=\pi$. Since $\wp(\cdot, z)$ varies as a continuous function of $S^{1}$, it then follows that the collection $\left\{w_{j}\left(t_{0}-\pi\right)\right\}$ is a permutation of the collection $\left\{w_{j}\left(t_{0}+\pi\right)\right\}$. This understood, there is a positive integer $p^{\prime} \leq n$ and a renumbering of the collection $\left\{w_{j}\right\}$ so that

$$
\begin{aligned}
w_{1}\left(t_{0}-\pi\right) & =w_{p^{\prime}}\left(t_{0}+\pi\right), \\
w_{2}\left(t_{0}-\pi\right) & =w_{1}\left(t_{0}+\pi\right), \\
& \vdots \\
w_{p^{\prime}}\left(t_{0}-\pi\right) & =w_{p^{\prime}-1}\left(t_{0}+\pi\right) .
\end{aligned}
$$

Define $w_{*}$ to be the $2 \pi p^{\prime}$-periodic function that is defined by concatenating the collection $\left\{w_{j}\right\}_{1 \leq j \leq p^{\prime}}$ at the points $\left\{t_{0}-\pi+2 j \pi\right\}_{j=0, \ldots, p^{\prime}}$. Then $w_{*}$ is a nontrivial, $2 \pi p^{\prime}$-periodic element in the kernel of the $(v, \mu)$ version of (1-2). However, no such solutions exist by assumption.

\section{Properties of instantons}

This section with Section 4 and Section 5 present some basic properties of instanton solutions to (1-11). They serve as a resource for the subsequent proof of Theorem 1.2.

The purpose of this section is to summarize some general features of the spinor and curvature of an instanton solution to any given $\mu \in \Omega$ and large $r$ versions of (1-11). The metric is defined by a given contact 1 -form $a$ and an almost complex structure $J$ on the kernel of $a$. Unless stated to the contrary, the only assumption is that $d a(\cdot, J(\cdot))$ is symmetric and positive definite. It is also assumed in what follows that the $\mathcal{P}$-norm of $\mu$ is bounded by 1 . With this bound implicit, all constants that appear in this and subsequent sections are independent of $\mu$. (These constants will depend on $\mu$ to the extent that they increase from some positive lower bound if the $\mathcal{P}$-norm of $\mu$ is allowed to increase.) 
Assume in what follows that $\mathfrak{d}=(A, \psi=(\alpha, \beta)): \mathbb{R} \rightarrow \operatorname{Conn}(E) \oplus C^{\infty}(M ; \mathbb{S})$ is an instanton solution to (1-11). Note that much of what is done here is very similar to what is done from Sections 2-4 in the article $\mathrm{SW} \Rightarrow \mathrm{Gr}$ from [6].

\section{3.a The size of $\alpha, \beta$ and the curvature: Part 1}

The lemma below gives the 4-dimensional version of what is said by the first two bullets of Lemma 2.3.

Lemma 3.1 There exists $\kappa \geq 1$ with the following significance: Suppose that $r \geq \kappa$ and that $(A, \psi=(\alpha, \beta))$ is an instanton solution to (1-11). Then

- $|\alpha| \leq 1+\kappa r^{-1}$.

- $|\beta|^{2} \leq \kappa r^{-1}\left(1-|\alpha|^{2}\right)+\kappa^{2} r^{-2}$.

Proof of Lemma 3.1 View $X=\mathbb{R} \times M$ as a four dimensional Riemannian manifold so as to view the instanton equations as the Seiberg-Witten equations on $X$. As in Chapters $2 \mathrm{a}-2 \mathrm{~b}$ of the article $\mathrm{SW} \Rightarrow \mathrm{Gr}$ from [6], the Bochner-Weitzenboch formula for the $\mathbb{R} \times M$ Dirac operator $\partial / \partial s+D_{A}$ with (1-11) lead to differential equalities for $w=\left(1-|\alpha|^{2}\right)$ and $|\beta|^{2}$ of the following sort:

- $\frac{1}{2} d^{\dagger} d w+r|\alpha|^{2} w-\left|\nabla_{A} \alpha\right|^{2}+\mathfrak{e}_{w}=0$,

- $\frac{1}{2} d^{\dagger} d|\beta|^{2}+r|\alpha|^{2}|\beta|^{2}+r\left(1+|\beta|^{2}\right)|\beta|^{2}+\left|\nabla_{A} \beta\right|^{2}+\mathfrak{e}_{\beta}=0$,

where $\left|\mathfrak{e}_{w}\right| \leq c_{0}\left(|\alpha|^{2}+\left|\nabla_{A} \beta\right|^{2}+|\beta|^{2}\right)$ and $\left|\mathfrak{e}_{\beta}\right| \leq c_{0}\left(|\beta|^{2}+\left|\beta\|\alpha|+| \beta\| \nabla_{A} \alpha\right|\right)$. Here, $\nabla_{A}$ denotes the covariant derivative on sections over $\mathbb{R} \times M$ of the pullbacks of $E$ and $E \otimes K^{-1}$; it is defined by viewing $A$ and the canonical connection on $K^{-1}$ as connections on the respective pullbacks. As in Chapters $2 \mathrm{a}-2 \mathrm{c}$ of the article $\mathrm{SW} \Rightarrow \mathrm{Gr}$ from [6], the maximum principle is used with these inequalities to derive the bounds asserted by the lemma. The use of the maximum principle in this noncompact setting requires Lemma 2.3 to guarantee the bounds given by the lemma hold as $s \rightarrow \pm \infty$.

The next lemma speaks to the size of $(\partial / \partial s) A$ and $B_{A}$. Note that given Lemma 3.1's bound on $|\beta|^{2}$, the top equation in (1-11) yields the bound $\left|(\partial / \partial s) A+B_{A}\right| \leq$ $r\left(1-|\alpha|^{2}\right)+c_{0}$. The lemma that follows asserts both $|(\partial / \partial s) A|$ and $\left|B_{A}\right|$ are bounded by $c_{0} r$ given an extra assumption. To state this assumption, suppose for the moment that $\mathfrak{c}=(A, \psi)$ is any given pair in $\operatorname{Conn}(E) \times \mathbb{C}^{\infty}(M ; \mathbb{S})$. Let $\mathfrak{L}_{\mathfrak{c}}$ denote the corresponding version of (1-14). With this notation understood, an instanton solution $s \rightarrow \mathfrak{d}(s) \in \operatorname{Conn}(E) \times \mathbb{C}^{\infty}(M ; \mathbb{S})$ to $(1-11)$ gives the continuous family of operators 
$\left\{\mathfrak{L}_{\mathfrak{d}(s)}\right\}_{s \in \mathbb{R}}$. This family of operators has a unique $s \rightarrow-\infty$ limit, and a unique $s \rightarrow \infty$ limit and so there is a well defined spectral flow for this family, this denoted by $f_{\mathfrak{d}}$.

Note in this regard, that the notion of the spectral flow $f_{\mathfrak{d}}$ is ambiguous if either of the $s \rightarrow \pm \infty$ limits has nontrivial kernel. As the results that follow demand only a lower bound on $f_{\mathfrak{d}}$, the phrase " $f_{\mathfrak{d}} \geq x$ " for any given $x \in R$ is defined in the following way. Let $\mathfrak{c}_{-}$and $\mathfrak{c}_{+}$denote the respective $s \rightarrow-\infty$ and $s \rightarrow \infty$ limits of $\mathfrak{d}$. Then $f_{\mathfrak{d}} \geq x$ if there exist sequences $\left\{\mathfrak{c}_{-i}\right\}_{i=1,2, \ldots}$ and $\left\{\mathfrak{c}_{+i}\right\}_{i=1,2, \ldots}$ in $\operatorname{Conn}(E) \times \mathbb{C}^{\infty}(M ; \mathbb{S})$ with the following three properties: First they converge respectively to $\mathfrak{c}_{-}$and $\mathfrak{c}_{+}$. Second, each element defines a version of (1-11) with trivial kernel. To set the stage for the third requirement, fix, for each index $i$, a path in $\operatorname{Conn}(E) \times \mathbb{C}^{\infty}(M ; \mathbb{S})$ of the following sort: The path starts at $\mathfrak{c}_{-i}$ proceed to $\mathfrak{c}_{-}$along a short path to $\mathfrak{c}_{-}$, it then follows the path defined by $\mathfrak{d}$ to $\mathfrak{c}_{+}$, and it then proceeds to $\mathfrak{c}_{+i}$ via a short path. The notion of "short" is such that the sequence of paths converges in the $C^{\infty}$ topology as $i \rightarrow \infty$ to the constant path. With such a path chosen, here is the third requirement: The spectral flow for (1-11) along this path is at least $x$.

Introduce $\mathrm{F}_{\mathfrak{d}}$ to denote the minimum of $f_{\mathfrak{d}}$ and 0 when $c_{1}(\operatorname{det}(\mathbb{S}))$ is not a torsion class, and to denote 0 otherwise.

The upcoming lemma reintroduces the function $\mathfrak{a}$ on $\operatorname{Conn}(E) \times \mathbb{C}^{\infty}(M ; \mathbb{S})$ as depicted in $[8,(3-19)]$. By way of a reminder, the latter is defined in terms of the function $\mathrm{E}$ in (1-9) and functions $\mathfrak{c s}$ and $\mathfrak{e}_{\mu}$ on $\operatorname{Conn}(E)$ by the formula

$$
\mathfrak{a}=\frac{1}{2}(\mathfrak{c s}-r \mathrm{E})+\mathfrak{e}_{\mu}+r \int_{M} \psi^{\dagger} D_{A} \psi
$$

In this regard, the value of $\mathfrak{c s}$ at a given connection $A$ is defined as follows: Fix once and for all a connection $A_{E}$ on $E$ with harmonic curvature 2-form and write $A=A_{E}+\widehat{a}_{A}$ with $\hat{a}_{A}$ a section of $i T^{*} M$. Then

$$
\mathfrak{c s}(A)=-\int_{M} \hat{a}_{A} \wedge * d \widehat{a}_{A}-2 \int_{M} \hat{a}_{A} \wedge *\left(B_{E}+\frac{1}{2} B_{A_{K}}\right) .
$$

Meanwhile, $\mathfrak{e}_{\mu}(A)=i \int_{M} \mu \wedge * B_{A}$.

Let $\mathfrak{d}: \mathbb{R} \rightarrow \operatorname{Conn}(E) \times \mathbb{C}^{\infty}(M ; \mathbb{S})$ denote an instanton solution to (1-11). Introduce as notation $A_{\mathfrak{d}}=\left(\lim _{s \rightarrow-\infty} \mathfrak{a}\left(\left.\mathfrak{d}\right|_{s}\right)-\lim _{s \rightarrow \infty} \mathfrak{a}\left(\left.\mathfrak{d}\right|_{s}\right)\right)$. Note that $A_{\mathfrak{d}}$ is gauge invariant. It is a positive number except in the case when $\mathfrak{d}$ is $\mathbb{R}$-invariant.

Lemma 3.2 Given $T>1$, there exists $\kappa>1$ with the following significance: Suppose that $r \geq \kappa$ and that $\mathfrak{d}=(A, \psi)$ is an instanton solution to (1-11) with either $\mathrm{A}_{\mathfrak{d}}<r^{2} T$ or else $\mathrm{F}_{\mathfrak{d}} \geq-r^{2} T$. Then both $|(\partial / \partial s) A|$ and $\left|B_{A}\right|$ are bounded by $\kappa r$. 
The proof of Lemma 3.2 is given momentarily.

Note with regards to the subsequent applications of this lemma that the precise value chosen for the constant $T$ is of no significance. This understood, the proof of Lemma 3.2 and the statements of all subsequent applications in the rest of this article take $T=1$. A different choice for $T$ changes various constants, but otherwise does not change the conclusions.

The proof of Lemma 3.2 exploits a preliminary lemma that gives a uniform bound for the $L^{2}$ norm of $B_{A}$ on length 1 subcylinders of $\mathbb{R} \times M$.

Lemma 3.3 There exists a constant, $\kappa>1$ with the following significance: Suppose that $r \geq \kappa$ and that $\mathfrak{d}=(A, \psi)$ is an instanton solution to (1-11) with either $\mathrm{A}_{\mathfrak{d}} \leq r^{2}$ or $\mathrm{F}_{\mathfrak{d}} \geq-r^{2}$. Let $s$ denote any point in $\mathbb{R}$. Then

$$
\int_{[s, s+1] \times M}\left(\left|\frac{\partial}{\partial s} A\right|^{2}+\left|B_{A}\right|^{2}+r\left|\frac{\partial}{\partial s} \psi\right|^{2}+r\left|D_{A} \psi\right|^{2}\right) \leq \kappa r^{2} .
$$

Proof of Lemma 3.3 The proof has five steps.

Step 1 Introduce the 1-form

$$
\mathfrak{B}_{(A, \psi)}=B_{A}-r\left(\psi^{\dagger} \tau^{k} \psi-i a\right)-i * d \mu+\frac{1}{2} B_{A_{K}} .
$$

The next lemma says something about the $L^{2}$ norms of $(\partial / \partial s) A, \mathfrak{B}_{(A, \psi)},(\partial / \partial s) \psi$ and $D_{A} \psi$. The statement of the lemma uses $\mathfrak{c}_{-}=\left(A_{-}, \psi_{-}\right)$and $\mathfrak{c}_{+}=\left(A_{+}, \psi_{+}\right)$ to denote the respective $s \rightarrow-\infty$ and $s \rightarrow+\infty$ limits in $\operatorname{Conn}(E) \times \mathbb{C}^{\infty}(M ; \mathbb{S})$ of $(A, \psi)$.

Lemma 3.4 Suppose that $(A, \psi)$ is an instanton solution to some $r \geq 1$ version of (1-11). Let $s^{\prime} \geq s \in \mathbb{R}$. Then

$$
\frac{1}{2} \int_{\left[s, s^{\prime}\right] \times M}\left(\left|\frac{\partial}{\partial s} A\right|^{2}+\left|\mathfrak{B}_{(A, \psi)}\right|^{2}+2 r\left(\left|\frac{\partial}{\partial s} \psi\right|^{2}+\left|D_{A} \psi\right|^{2}\right)\right)=\mathfrak{a}\left(\left.\mathfrak{d}\right|_{s}\right)-\mathfrak{a}\left(\left.d\right|_{s^{\prime}}\right) .
$$

Moreover,

$$
\frac{1}{2} \int_{\mathbb{R} \times M}\left(\left|\frac{\partial}{\partial s} A\right|^{2}+\left|\mathfrak{B}_{(A, \psi)}\right|^{2}+2 r\left(\left|\frac{\partial}{\partial s} \psi\right|^{2}+\left|D_{A} \psi\right|^{2}\right)\right)=\mathfrak{a}\left(\mathfrak{c}_{-}\right)-\mathfrak{a}\left(\mathfrak{c}_{+}\right) .
$$

Proof of Lemma 3.4 Integrate the sum of norm squared of the top equation in (1-11) with $2 r$ times the norm squared of the lower equation. Integration by parts yields the lemma's assertion.

The next lemma bounds $\mathfrak{a}\left(\mathfrak{c}_{-}\right)-\mathfrak{a}\left(\mathfrak{c}_{+}\right)$in terms of $F_{\mathfrak{d}}$ and the respective values on $\mathfrak{c}_{-}$ and $\mathfrak{c}_{+}$of the function $\mathrm{E}$ in (1-9). 
Lemma 3.5 There exists a constant $\kappa \geq 1$ with the following significance: Suppose that $r \geq \kappa$ and that $(A, \psi)$ is an instanton solution to (1-11). Then

$$
\begin{aligned}
\mathfrak{a}\left(\mathfrak{c}_{-}\right)-\mathfrak{a}\left(\mathfrak{c}_{+}\right) \leq-2 \pi^{2} \mathrm{~F}_{\mathfrak{d}}+\frac{1}{2} r \mathrm{E}\left(A_{+}\right) & -\frac{1}{2} r \mathrm{E}\left(A_{-}\right) \\
& +\kappa r^{2 / 3}(\ln )^{k}\left(\left|\mathrm{E}\left(A_{+}\right)\right|^{4 / 3}+\left|\mathrm{E}\left(A_{-}\right)\right|^{4 / 3}\right)+\kappa .
\end{aligned}
$$

However, in the case when $c_{1}(\operatorname{det}(\mathbb{S}))$ is torsion,

$$
\mathfrak{a}\left(\mathfrak{c}_{-}\right)-\mathfrak{a}\left(\mathfrak{c}_{+}\right) \leq \frac{1}{2} r \mathrm{E}\left(A_{+}\right)-\frac{1}{2} r \mathrm{E}\left(A_{-}\right)+\kappa r^{2 / 3}\left(\left|\mathrm{E}\left(A_{+}\right)\right|^{4 / 3}+r^{2 / 3}\left|\mathrm{E}\left(A_{-}\right)\right|^{4 / 3}\right)+\kappa .
$$

Proof of Lemma 3.5 This follows from [8, Proposition 4.10].

These last two lemmas imply the following:

$$
\begin{aligned}
\int_{\mathbb{R} \times M}\left(\left|\frac{\partial}{\partial s} A\right|^{2}+\left|\mathfrak{B}_{(A, \psi)}\right|^{2}+2 r\left(\left|\frac{\partial}{\partial s} \psi\right|^{2}+\left|D_{A} \psi\right|^{2}\right)\right) & \\
& \leq-2 \pi \mathrm{F}_{\mathfrak{d}}+c_{0} r^{2}(\ln r)^{c_{0}}
\end{aligned}
$$

Step 2 Assume that either $\mathrm{A}_{\mathfrak{d}}<r^{2}$ or $\mathrm{F}_{\mathfrak{d}}>-r^{2}$. When $k \in \mathbb{Z}$, let $I_{k}=[k, k+1]$. It is a consequence of Lemma 3.4 and (3-5) that there are at most $c_{0}(\operatorname{lnr})^{c_{0}}$ integers $k \in \mathbb{Z}$ where

$$
\int_{I_{k} \times M}\left(\left|\frac{\partial}{\partial s} A\right|^{2}+\left|\mathfrak{B}_{(A, \psi)}\right|^{2}+2 r\left(\left|\frac{\partial}{\partial s} \psi\right|^{2}+\left|D_{A} \psi\right|^{2}\right)\right) \geq r^{2} .
$$

In particular, given any $k \in \mathbb{Z}$, there exists $T \leq c_{0}(\operatorname{lnr})^{c_{0}}$ such that

$$
\int_{I_{k \pm T} \times M}\left(\left|\frac{\partial}{\partial s} A\right|^{2}+\left|\mathfrak{B}_{(A, \psi)}\right|^{2}+2 r\left(\left|\frac{\partial}{\partial s} \psi\right|^{2}+\left|D_{A} \psi\right|^{2}\right)\right) \leq r^{2} .
$$

This last inequality has the following consequence: There exist points $s \in I_{k-T}$ and points $s \in I_{k+T}$ such that

$$
\int_{\{s\} \times M}\left(\left|B_{A}\right|^{2}+\left|D_{A} \psi\right|^{2}\right) \leq r^{2}
$$

Step 3 Fix a connection $A_{E} \in \operatorname{Conn}(E)$ with harmonic curvature 2-form. Let $A$ denote for the moment any given element in $\operatorname{Conn}(E)$ and write $A=A_{E}+\widehat{a}_{A}$. Use $\mathfrak{p}\left(\hat{a}_{A}\right)$ in what follows to denote the value on the Poincare dual of $c_{1}(\operatorname{det}(\mathbb{S}))$ of the $L^{2}$-orthogonal projection of $\hat{a}$ into the space of $i \mathbb{R}$-valued harmonic 1 -forms on $M$. Introduce the Chern-Simon's function $\mathfrak{c s}$ as defined in (3-3). Then

$$
|\mathfrak{c s}(A)| \leq c_{0}\left|p\left(\widehat{a}_{A}\right)\right|+c_{0}\left\|B_{A}\right\|_{2}^{2} .
$$


Now suppose that $\psi \in C^{\infty}(M ; \mathbb{S})$. It follows as a consequence of (3-9) that

$$
|\mathfrak{a}(A, \psi)| \leq c_{0}\left(\left|\mathfrak{p}\left(\hat{a}_{A}\right)\right|+\left\|B_{A}\right\|_{2}^{2}+r\left\|B_{A}\right\|_{2}+r\|\psi\|_{2}\left\|D_{A} \psi\right\|_{2}\right) .
$$

Step 4 Let $k$ and $T$ be as in Step 2. Choose $s_{-} \in I_{k-T}$ and $s_{+} \in I_{k+T}$ so that (3-8) holds with $s=s_{-}$and with $s=s_{+}$. There exists a map $u: M \rightarrow S^{1}$ so that $\left.A\right|_{s_{-}}-u^{-1} d u$ can be written as $A_{E}+\widehat{a}$ where $\hat{a}$ is coclosed and such that $|\mathfrak{p}(\hat{a})| \leq c_{0}$. Extend $\hat{a}$ as a section of $i T^{*} M$ over the cylinder $\left[s_{-}, s_{+}\right] \times M$ by writing $A-u^{-1} d u$ as $A_{E}+\hat{a}$. With this extension understood, it follows from the top line in (1-11) that

$$
\left|\frac{d}{d s} \mathfrak{p}(\widehat{a})\right| \leq c_{0} r
$$

at each $s \in\left[s_{-}, s_{+}\right]$. This implies that

$$
\left|\mathfrak{p}\left(\left.\widehat{a}\right|_{s_{+}}\right)\right| \leq c_{0} r(\operatorname{lnr})^{c_{0}} .
$$

Step 5 Take $s=s_{-}$and $s^{\prime}=s_{+}$in the first equality of Lemma 3.4. Then use (3-9) at $s=s_{-}$and at $s=s_{+}$with (3-10) to see that

$$
\int_{\left[s_{-}, s_{+}\right] \times M}\left(\left|\frac{\partial}{\partial s} A\right|^{2}+\left|\mathfrak{B}_{(A, \psi)}\right|^{2}+2 r\left(\left|\frac{\partial}{\partial s} \psi\right|^{2}+\left|D_{A} \psi\right|^{2}\right)\right) \leq c_{0} r^{2} .
$$

As a consequence

$$
\int_{I_{k} \times M}\left(\left|\frac{\partial}{\partial s} A\right|^{2}+\left|\mathfrak{B}_{(A, \psi)}\right|^{2}+2 r\left(\left|\frac{\partial}{\partial s} \psi\right|^{2}+\left|D_{A} \psi\right|^{2}\right)\right) \leq c_{0} r^{2} .
$$

This implies the assertion of Lemma 3.3 because $\left|\mathfrak{B}_{(A, \psi)}\right|^{2} \geq \frac{1}{2}\left|B_{A}\right|^{2}-c_{0} r^{2}$.

Proof of Lemma 3.2 Use (3-1) with the arguments used to prove Lemma 2.5 in the article $\mathrm{SW} \Rightarrow$ Gr from [6] to derive constants $z_{1}, z_{2}, z_{3}$, and $z_{4}$ with two salient features. First, they are $(A, \psi)$ and $r$ independent. To state the second, introduce the function

$$
q_{0}=r\left(1+r^{-1} z_{1}\right)\left(1-|\alpha|^{2}\right)-z_{2} r|\beta|^{2}+z_{3} .
$$

Also, introduce $s$ to denote $\left|(\partial / \partial s) A-B_{A}\right|$ and set $q=\max \left(s-q_{0}, 0\right)$. Here is the second feature: The function $q$ obeys

$$
d^{\dagger} d q+2 r|\alpha|^{2} q \leq z_{4}\left(s+r\left(1-|\alpha|^{2}\right)\right) \text {. }
$$

Here and until said otherwise, $d$ denotes the exterior derivative for functions on $\mathbb{R} \times M$ and $d^{\dagger}$ denotes its formal $L^{2}$ adjoint. Thus, $d^{\dagger} d$ is minus the metric Laplacian on $\mathbb{R} \times M$. (The factor 2 that multiplies $r|\alpha|^{2}$ above and in subsequent equations differs 
from the factor multiplying $r|\alpha|^{2}$ in the article SW $\Rightarrow$ Gr from [6]. These differing factors have no bearing in what follows as they can be reconciled with a redefinition of $r$.) Equation (3-16) implies that

$$
d^{\dagger} d q-z_{4} q \leq c_{0} r\left(1-|\alpha|^{2}\right)+c_{0} .
$$

To make something of this, fix $\rho>0$ but less than one fourth the injectivity radius of $M$. Fix $x \in M$ and let $B \subset \mathbb{R} \times M$ denote the ball of radius $\rho$ with center at $B$. If $\rho \leq c_{0}^{-1}$, then the operator $d^{\dagger} d-z_{4}$ on $B$ with Dirichlet boundary conditions has purely positive spectrum with smallest eigenvalue greater than $c_{0}^{-1}$. Fix $\rho$ so that such is the case. Let $G_{0}(\cdot, x)$ denote the Green's function with pole at $x$. The maximum principle implies

$$
0 \leq G(\cdot, x) \leq c_{0} \frac{1}{\operatorname{dist}(\cdot, x)^{2}} \quad \text { and } \quad|d G(\cdot, x)| \leq c_{0} \frac{1}{\operatorname{dist}(\cdot, x)^{3}} .
$$

Multiply both sides of (3-17) by $\chi(\operatorname{dist}(\cdot, x) / \rho) G_{0}(\cdot, x)$ and integrate over $B$. Integrate by parts and invoke (3-18) to see that

$$
q(x) \leq c_{0} \rho^{-2}\left(\int_{B}\left(\left|\frac{\partial}{\partial s} A\right|^{2}+\left|B_{A}\right|^{2}\right)\right)^{1 / 2}+c_{0} r \rho^{2} .
$$

Lemma 3.3 and this inequality imply that $s \leq r\left(1+r^{-1} z_{1}\right)\left|\left(1-|\alpha|^{2}\right)\right|+c_{0} r$. In particular, $s \leq c_{0} r$; and this gives the claim made by Lemma 3.2.

\section{3.b The size of $\alpha, \beta$ and the curvature: Part 2}

This part refines the bounds given by Lemmas 3.1 and 3.2. The next lemma speaks to the size of the covariant derivatives of $\alpha$ and $\beta$. This lemma and, unless noted to the contrary, the subsequent discussions use $\nabla_{A}$ to denote the covariant derivative of a section of a bundle over $\mathbb{R} \times M$ as defined by viewing $A$ as a connection on the pullback of $E$ over $\mathbb{R} \times M$. In particular, $\nabla_{A}$ has a component that differentiates along the $\mathbb{R}$ factor of $\mathbb{R} \times M$.

Lemma 3.6 There exists $\kappa \geq 1$ with the following significance: Suppose that $r \geq \kappa$ and that $\mathfrak{d}=(A, \psi=(\alpha, \beta))$ is an instanton solution to (1-11) with $\mathrm{A}_{\mathfrak{d}}<r^{2}$ or $\mathrm{F}_{\mathfrak{d}} \geq-r^{2}$. Then

- $\left|\nabla_{A} \alpha\right|^{2} \leq \kappa r$.

- $\left|\nabla_{A} \beta\right|^{2} \leq \kappa$.

In addition, for each $q \geq 1$, there exists a constant $\kappa_{q}$ which is such that when $r \geq \kappa$ then

$$
\text { - }\left|\nabla_{A}^{q} \alpha\right|+r^{1 / 2}\left|\nabla_{A}^{q} \beta\right| \leq \kappa_{q} r^{q / 2} \text {. }
$$


Proof of Lemma 3.6 These claims are local in nature and are proved by rescaling the Seiberg-Witten equation as written in Gaussian normal coordinates about any given point. Here and elsewhere in this article, these are coordinates which make the metric look like the Euclidean metric up to an error that is proportional to the square of the distance from the point in question. The rescaling writes the Gaussian coordinate functions $\left\{x^{v}\right\}_{v=1,2,3,4}$ as $x^{v}=r^{-1 / 2} y^{v}$. Uniform bounds on the curvatures in the rescaled coordinates follow from Lemma 3.2. This understood, uniform bounds on the covariant derivatives of the rescaled sections can be obtained using standard elliptic regularity techniques. Undoing the rescaling gives the asserted bounds.

Lemma 3.7 There exists $\kappa>1$ with the following significance: Suppose that $r \geq \kappa$ and that $\mathfrak{d}=(A, \psi=(\alpha, \beta))$ is an instanton solution to (1-11) with $\mathrm{A}_{\mathfrak{d}}<r^{2}$ or $\mathrm{F}_{\mathfrak{d}} \geq-r^{2}$. Define the function $s \rightarrow \underline{\mathrm{M}}(S)=r \int_{[s-1, s+1] \times M}\left(1-|\alpha|^{2}\right)$ on $\mathbb{R}$. Suppose that $s_{0} \in \mathbb{R}$, that $R \geq 1$, and that $\mathcal{K} \geq 1$ are such that $\sup _{\left[s_{0}-R-2, s_{0}+R+2\right]} \underline{\mathrm{M}}(\cdot) \leq \mathcal{K}$. Then

$$
\left|\frac{\partial}{\partial s} A-B_{A}\right| \leq r\left(1+\kappa \mathcal{K}^{1 / 2} r^{-1 / 2}\right)\left(1-|\alpha|^{2}\right)+\kappa
$$

at all points where $s \in\left[s_{0}-R, s_{0}+R\right]$.

Proof of Lemma 3.7 The proof is has four steps.

Step 1 Reintroduce the function $q$ from the proof of Lemma 3.2. It follows from (3-16) and just stated bound on $s$ that $z_{5} \geq 1$ can be chosen independent of $(A, \psi)$ and $r$ so that $q_{1}=\max \left(q-z_{5}, 0\right)$ obeys

$$
d^{\dagger} d q_{1}+2 r|\alpha|^{2} q_{1} \leq c_{0} r\left(1-|\alpha|^{2}\right) .
$$

Write this equation as

$$
d^{\dagger} d q_{1}+2 r q_{1} \leq c_{0} r\left(1-|\alpha|^{2}\right)+2 r\left(1-|\alpha|^{2}\right) q_{1} .
$$

Let $y \in\left[s_{0}-R-\frac{3}{2}, s_{0}+R+\frac{3}{2}\right]$ and let $q_{y}$ denote the function $\chi(4|s(\cdot)-y|-1) q_{1}$. Note that $q_{y}$ has support only where $|s-y| \leq \frac{1}{2}$. Multiply both sides of (3-21) by the bump function $\chi(4|s(\cdot)-y|-1)$ to obtain a differential inequality for $q_{y}$. Integrate this inequality using the fact that both $\mathfrak{s}$ and $q$ are bounded by $c_{0} r$ to see that $\left\|q_{y}\right\|_{L^{1}} \leq c_{0}(1+\mathcal{K})$.

Step 2 Let $\rho=c_{0}^{-1}$ denote a fixed constant that is much less than $M$ 's injectivity radius. Given $y \in\left[s_{0}-R-\frac{3}{2}, s_{0}+R+\frac{3}{2}\right]$, use $q_{y}$ to denote now $\chi\left(\rho^{-1} \operatorname{dist}(\cdot, y)\right) q_{1}$. Multiply both sides (3-20) by the function $\chi\left(\rho^{-1} \operatorname{dist}(\cdot, y)\right)$ to obtain a differential inequality for $q_{y}$. For $x \in \mathbb{R} \times M$, let $G(\cdot, x)$ now denote the Dirichlet Green's 
function for the operator $d^{\dagger} d$ on the ball of radius $\rho$ centered at $x$. This Green's function is also described by (3-18). Use $G(\cdot, y)$ as in the proof of Lemma 3.2 with the aforementioned differential inequality for $q_{y}$ to bound the norm of $q_{y}(y)$ by

$$
\left|q_{y}(y)\right| \leq c_{0}\left(1+\mathcal{K}+(\mathcal{K} r)^{1 / 2}\right) .
$$

To elaborate, a factor $c_{0}(1+\mathcal{K})$ comes from the integration over the set of points where $d \chi\left(\rho^{-1} \operatorname{dist}(\cdot, y)\right) \neq 0$ of factors that are products of $\rho^{-2} G(\cdot, y) q_{y}$ and $\rho^{-1}|d G(y, \cdot)| q_{y}$. What with (3-18), these contribute at most $\rho^{-4} c_{0}\left\|q_{y}\right\|_{L^{1}}$. The factor $c_{0}(\mathcal{K} r)^{1 / 2}$ arises from the integral of $G(\cdot, y) r\left(1-|\alpha|^{2}\right)$. Given $d \in(0, \rho)$, the latter is bounded by breaking the integrand into the part where $\operatorname{dist}(\cdot, y) \geq d$ and where $\operatorname{dist}(\cdot, y) \leq d$. The former contributes at most $\mathcal{K} d^{-2}$ and the latter at most $r d^{2}$. Taking $d=\mathcal{K}^{1 / 2} r^{-1 / 2}$ gives the bound by $c_{0}(\mathcal{K} r)^{1 / 2}$ of these two contributions.

The various $y \in\left[s_{0}-R-\frac{3}{2}, s_{0}+R+\frac{3}{2}\right]$ versions of (3-22) together supply the bound

$$
q_{1} \leq c_{0}\left(1+\mathcal{K}+(\mathcal{K} r)^{1 / 2}\right)
$$

at all points in $\left[s_{0}-R-1, s_{0}+R+1\right] \times M$.

Step 3 Let $x=\cosh \left(\frac{1}{2} r^{1 / 2}\left(s-s_{0}\right)\right) / \cosh \left(\frac{1}{2} r^{1 / 2}(R+1)\right)$. This function obeys the equation

$$
-\frac{d^{2} x}{d s^{2}}+\frac{1}{4} r x=0
$$

on $\left[s_{0}-R-1, s_{0}+R+1\right]$ with value 1 at $s=s_{0} \pm R$. Note that $x \leq\left\|q_{1}\right\|_{\infty}$ on $\left[s_{0}-R-1, s_{0}+R+1\right]$; and $x \leq r^{-10}$ on $\left[s_{0}-R, s_{0}+R\right] \times M$ if $r \geq c_{0}$.

To continue, use (3-1) with what is done in the discussion surrounding Equation (2-28) in Section $2 \mathrm{~d}$ of the first article $\mathrm{SW} \Rightarrow \mathrm{Gr}$ in [6] to find constants $z_{5}, z_{6}$ that have the following properties: Both are positive, and $v_{1}=\left(1-|\alpha|^{2}\right)+z_{5} r^{-1}-z_{6}|\beta|^{2}$ obeys

- $v_{1} \geq c_{0} r^{-1}$.

- $v_{1} \geq\left(1-|\alpha|^{2}\right)$.

- $d^{\dagger} d v_{1}+\frac{1}{4} r v_{1} \geq 0$ where $|\alpha|^{2} \geq \frac{1}{2}$.

Set $\varepsilon=r^{-1 / 2}$ and note that $v_{2}=v_{1}^{1-\varepsilon}$ obeys $v_{2} \geq\left(1-|\alpha|^{2}\right)$, and it obeys the equation

$$
d^{\dagger} d v_{2}+\frac{1}{4} r v_{2} \geq \frac{1}{8} r^{1 / 2}\left(1-|\alpha|^{2}\right)
$$

where $|\alpha|^{2} \geq \frac{1}{2}$. 
Step 4 Step 3 implies that $q_{2}=q_{1}-c_{0}\left(1+r^{-1 / 2}\left\|q_{1}\right\|_{\infty}\right) r^{1 / 2}\left(v_{2}+x\right)$ obeys

$$
d^{\dagger} d q_{2}+\frac{1}{4} r q_{2} \leq 0
$$

on the subset $U \subset\left[s_{0}-R-1, s_{0}+R+1\right] \times M$ where $|\alpha|^{2} \geq \frac{1}{2}$ and it is nonpositive on the boundary of this subset. The maximum principle asserts that $q_{2}$ is negative on $U$.

The inequality asserted by the second bullet in Lemma 3.2 for points in $U$ follows using (3-23), the fact that $q_{2} \leq 0$ in $U$, and the bound by $r^{-10}$ on the values of $x$ at points in $\left[s_{0}-R, s_{0}+R\right]$. The inequality for points in the complement of $U$ follows from (3-23) and the fact that $\left(1-|\alpha|^{2}\right)$ is greater than $\frac{1}{2}$ on this complement.

\section{3.c The size of $\alpha, \beta$ and the curvature: Part 3}

The bounds given in Lemma 3.7 are used here to further refine the a priori bounds on $\alpha, \beta$ and the curvature. The first lemma below states the analog here of what is asserted by [6, Propositions 2.8 and 4.4 of the article $\mathrm{SW} \Rightarrow \mathrm{Gr}$ ].

Lemma 3.8 Given $\mathcal{K} \geq 1$, there exists $\kappa \geq 1$ with the following significance: Suppose that $r \geq \kappa$, and that $(A, \psi=(\alpha, \beta))$ is an instanton solution to (1-11) with either $\mathrm{A}_{\mathfrak{d}}<r^{2}$ or $\mathrm{F}_{\mathfrak{d}} \geq-r^{2}$. Fix a point $s_{0} \in \mathbb{R}$ and $R \geq 2$; and suppose that

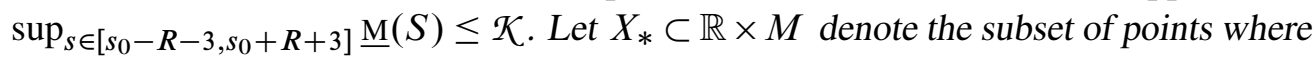
$1-|\alpha| \geq \kappa^{-1}$. The bounds stated below hold on the domain $\left[s_{0}-R, s_{0}+R\right] \times M$.

- $\left|\nabla_{A} \alpha\right|^{2}+r\left|\nabla_{A} \beta\right|^{2} \leq \kappa r\left(1-|\alpha|^{2}\right)+\kappa^{2}$.

- $r\left(1-|\alpha|^{2}\right)+\left|\nabla_{A} \alpha\right|^{2}+r\left|\nabla_{A} \beta\right|^{2} \leq \kappa\left(r^{-1}+r e^{-\sqrt{r} \operatorname{dist}\left(\cdot, X_{*}\right) / \kappa}\right)$.

- $|\beta|^{2} \leq \kappa\left(r^{-2}+r^{-1} e^{-\sqrt{r} \operatorname{dist}\left(\cdot, X_{*}\right) / \kappa}\right)$.

As in Lemma 3.6, what is written as $\nabla_{A}$ refers to the covariant derivative over $\mathbb{R} \times M$ as defined by viewing $A$ as a connection on the pullback of the bundle $E$ to $\mathbb{R} \times M$.

Proof of Lemma 3.8 What with Lemma 3.7, the manipulations done in Step 2 from the proof of Proposition 4.4 in the article SW $\Rightarrow$ Gr in [6] can be copied to obtain the following: There exists an $r$ and $(A, \psi)$ independent constant $z_{6} \geq 1$ such that the function $y=\left|\nabla_{A} \alpha\right|^{2}+r\left|\nabla_{A} \beta\right|^{2}-z_{6}$ obeys the differential inequality

$$
d^{\dagger} d y+2 r|\alpha|^{2} y \leq c_{0} r\left(1-|\alpha|^{2}\right) y+c_{0} y
$$

at points in $\left[s_{0}-R-1, s_{0}+R+1\right] \times M$ where $1-|\alpha|^{2} \leq c_{0}^{-1}$. Meanwhile, use (3-1) with Lemma 3.1 and Lemma 3.6 to obtain an $(A, \psi)$ and $r$ independent constant $z_{7}$ such that the function $w=\left(1-|\alpha|^{2}\right)-z_{7}|\beta|^{2}$ obeys

$$
-c_{0}+y \leq d^{\dagger} d w+2 r|\alpha|^{2} w \leq c_{0}(y+1) .
$$


Let $s \rightarrow x(s)$ again denote the function $\cosh \left(\frac{1}{2} r^{1 / 2}\left(s-s_{0}\right)\right) / \cosh \left(\frac{1}{2} r^{1 / 2}(R+1)\right)$. Fix $c_{*} \geq 1$ and define $u=\max \left(y-c_{0}\left(\|y\|_{\infty}+c_{0}\right)(w+x)-c_{0}, 0\right)$. If $c_{*} \geq c_{0}$, it follows from (3-27) with the leftmost inequality in (3-28) that $u$ obeys the differential inequality

$$
d^{\dagger} d u+\frac{1}{64} r u \leq 0
$$

on the domain $U \subset\left[s_{0}-R-1, s_{0}+R+1\right] \times M$ where $1-|\alpha|^{2} \leq c_{0}^{-1}$ and that $u$ is zero on the boundary of $U$. This understood, the maximum principle finds $u=0$ in the whole of $U$. What with Lemma 3.6, this proves the assertion of the first bullet of the lemma for points in $U$. Meanwhile, Lemma 3.6 and the fact that $w>c_{0}^{-1}$ on the complement of $U$ imply the assertion of the first bullet on the complement of $U$.

To obtain the assertion of the second bullet, use (3-27) with the left hand inequality in (3-28) to see that $u^{\prime}=\max \left(y+c_{0}^{-1} r w-c_{0}, 0\right)$ obeys $d^{\dagger} d u^{\prime}+\frac{1}{64} r u^{\prime} \leq 0$ in $U$. Keeping this in mind, let $c_{M}>0$ denote a constant that is much less than the injectivity radius of $M$. Let $x \in X_{*}$ denote a point with $s(x) \in\left[s_{0}-R, s_{0}+R\right]$, and let $B \subset X_{*}$ denote the ball with center $x$ and radius equal to half of the minimum of $c_{M}$ and $\operatorname{dist}\left(x, X_{*}\right)$. Use $\rho$ to denote the radius of the ball $B$. Let $G(\cdot, x)$ denote the Green's function for the operator $d^{\dagger} d+\frac{1}{64} r$ with pole at $x$. This operator obeys the bounds

$$
\begin{aligned}
0 \leq G(\cdot, x) & \leq c_{0} \frac{1}{\operatorname{dist}(\cdot, x)} e^{-\sqrt{r} \operatorname{dist}(\cdot, x) / c_{0}}, \\
|d G(\cdot, x)| & \leq c_{0} \frac{1}{\operatorname{dist}(\cdot, x)} e^{-\sqrt{r} \operatorname{dist}(\cdot, x) / c_{0}} .
\end{aligned}
$$

Multiply both sides of the inequality $d^{\dagger} d u^{\prime}+\frac{1}{64} r u^{\prime} \leq 0$ by $\chi(\operatorname{dist}(\cdot, x) / \rho) G(\cdot, x)$ and integrate by over $B$. Given that $\left|u^{\prime}\right| \leq c_{0} r$, integration by parts finds that $\left.u^{\prime}\right|_{x} \leq$ $c_{0} r e^{-\sqrt{r} \rho / c_{0}}$.

This implies what is asserted by the second bullet of the lemma. The third bullet follows from the second using Lemma 3.1.

The next lemma refines the bounds given by Lemma 3.7 for the curvature.

Lemma 3.9 Given or $\mathcal{K} \geq 1$, there exists $\kappa \geq 1$ with the following significance: Suppose that $r \geq \kappa$, and that $(A, \psi=(\alpha, \beta))$ is an instanton solution to (1-11) with either $\mathrm{A}_{\mathfrak{d}}<r^{2}$ or $\mathrm{F}_{\mathfrak{d}} \geq-r^{2}$. Fix a point $s_{0} \in \mathbb{R}$ and $R \geq 1$; and suppose that 
$\sup _{s \in\left[s_{0}-R-3, s_{0}+R+3\right]} \underline{\mathrm{M}}(s) \leq \mathcal{K}$. Then

$$
\begin{aligned}
&-\left|\frac{\partial}{\partial s} A+B_{A}\right| \leq r\left(1-|\alpha|^{2}\right)+\kappa \\
& \text { - }\left|\frac{\partial}{\partial s} A-B_{A}\right| \leq r\left(1-|\alpha|^{2}\right)+\kappa
\end{aligned}
$$

at all points in $\left[s_{0}-R, s_{0}+R\right] \times M$.

The proof of this lemma requires a second result, one of great importance in its own right. This is the analog of the monotonicity formula stated by Proposition 3.1 of the article $\mathrm{SW} \Rightarrow \mathrm{Gr}$ in [6].

Lemma 3.10 Given $\mathcal{K} \geq 1$, there exists $\kappa>1$ with the following significance: Take $r \geq \kappa$ and let $\mathfrak{d}=(A, \psi=(\alpha, \beta))$ be an instanton solution to (1-11) with either $\mathrm{A}_{\mathfrak{d}}<r^{2}$ or $\mathrm{F}_{\mathfrak{d}} \geq-r^{2}$. Fix $s_{0} \in \mathbb{R}$ and suppose that $\sup _{\left[s_{0}-4, s_{0}+4\right]} \underline{\mathrm{M}}(\cdot) \leq \mathcal{K}$. Given $x \in\left[s_{0}-R-1, s_{0}+R+1\right] \times M$ and given a number $\rho \in\left(r^{-1 / 2}, \kappa^{-1}\right)$, use $\mathrm{M}(x, \rho)$ to denote the integral of $r\left(1-|\alpha|^{2}\right)$ over the radius $\rho$ ball in $\mathbb{R} \times M$ centered at $x$. Then

- If $\rho_{1}>\rho_{0}$ are in $\left(r^{-1 / 2}, \kappa^{-1}\right)$, then $\mathrm{M}\left(x, \rho_{1}\right)>\kappa^{-1} \rho_{1}^{2} / \rho_{0}^{2} \mathrm{M}\left(x, \rho_{0}\right)$.

- $\mathrm{M}(x, \rho) \leq \kappa \mathcal{K} \rho^{2}$.

- Suppose that $|\alpha| \leq \frac{1}{2}$ at $x$. If $\rho \in\left(r^{-1 / 2}, \kappa^{-1}\right)$, then $\operatorname{M}(x, \rho) \geq \kappa^{-1} \rho^{2}$.

Proof of Lemma 3.10 Lemma 3.7 holds on the ball of radius $c_{0}^{-1}$ centered at $x$ by virtue of what is assumed about $\underline{\mathrm{M}}(\cdot)$. This being the case, the proof of Proposition 3.1 in the article $\mathrm{SW} \Rightarrow \mathrm{Gr}$ from [6] can be repeated here with only cosmetic changes to prove the first item. The second follows from the first given the bound on $\underline{M}$. The third follows from the first given that $\mathrm{M}\left(x, r^{-1 / 2}\right) \geq c_{0} r$. To prove the latter assertion, it is sufficient to note that if $|\alpha|(x) \leq \frac{1}{2}$, then it follows using the first item of Lemma 3.6 that $|\alpha| \leq \frac{3}{4}$ in the ball of radius $c_{0}^{-1} r^{-1 / 2}$ centered at $x$.

Proof of Lemma 3.9 The first assertion follows directly from (1-11) using Lemma 3.1. To obtain the second assertion, reintroduce the function $q_{1}$ from Step 1 of the proof of Lemma 3.7. What is asserted by Lemma 3.9 follows if $q_{1}$ can be bounded by an $(A, \psi)$ and $r$ independent constant at points in $\left[s_{0}-R, s_{0}+R\right] \times M$. To see that this is the case, fix a point $x$ in this domain and let $B$ denote a ball of radius $\rho=c_{0}^{-1}$ centered at $x$, with $\rho$ chosen to be much less than the injectivity radius of $M$. Let $z_{x}$ denote the function with compact support on $B$ given by $\chi\left(\rho^{-1} \operatorname{dist}(x, \cdot)\right)$. Let $\mathfrak{x}$ denote the function with compact support on $B$ that obeys

$$
d^{\dagger} d \mathfrak{x}+\frac{1}{64} r \mathfrak{x}=d^{\dagger} d z_{x} q_{1}-2\left\langle d z_{x}, d q_{1}\right\rangle .
$$


Here, $\langle$,$\rangle is used to denote the metric inner product. Given that the Dirichlet Green's$ function for $d^{\dagger} d+\frac{1}{64} r$ in $B$ obeys (3-30), and given that $\left|q_{1}\right| \leq c_{0} r^{1 / 2}\left(1-|\alpha|^{2}\right)+c_{0}$, it follows that $|x|(x) \leq c_{0}$. Note that these last three versions of the constant $c_{0}$ depend on $\mathcal{K}$, as do the subsequent appearances of $c_{0}$ in this proof. However, they are otherwise independent of $(A, \psi)$ and $r$. Granted this, it follows from (3-20) that $q_{x}=z_{x} q_{1}-\mathfrak{x}$ obeys

$$
d^{\dagger} d q_{x}+2 r|\alpha|^{2} q_{x} \leq c_{0} r \quad \text { in } B
$$

By construction, $q_{x}=0$ on $\partial B$.

Given Lemma 3.10, the proof of Lemma 3.5 of the article SW $\Rightarrow$ Gr in [6] can be copied almost verbatim to obtain a positive function $u$ on $B$ that obeys the following three conditions: First, $u \leq c_{0}$, a constant that depends on $\mathcal{K}$, but not $r$. Second, $d^{\dagger} d u \geq r$ at points where $|\alpha| \leq 1 / 2$. Third, $\left|d^{\dagger} d u\right| \leq c_{0} r$ on the whole of $B$. Granted these conditions, it follows that the function $v=q_{x}-c u-c_{0}$ is such that $d^{\dagger} d v+2 r|\alpha|^{2} v \leq 0$ in $B$. Since $v \leq 0$ on $\partial B$, the maximum principle demands that $v \leq 0$ in $B$. What with $u \leq c_{0}$ and $\mathfrak{x}(x) \leq c_{0}$ this means that $\left.q_{1}\right|_{x} \leq c_{0}$.

Together, Lemma 3.8 and Lemma 3.9 assert only that the curvature $B_{A}$ is $O(1)$ at points where $|\alpha|$ is nearly 1 . To say more about this, let $F_{A}=d s \wedge(\partial / \partial s) A+* B_{A}$ where $*$ here denotes the Hodge star along the $M$ factor in $\mathbb{R} \times M$. This 2-form $F_{A}$ is the associated curvature 2 -form that comes by viewing $E$ as a bundle over $\mathbb{R} \times M$ and $A$ as a connection on this $E \rightarrow \mathbb{R} \times M$. Suppose that $\wp:[0, \infty) \rightarrow[0, \infty)$ is a smooth, nondecreasing function which obeys $\wp(x)=x$ for $x$ near 0 and $\wp(1)=1$. With $\wp$ chosen, set

$$
\widehat{A}=A-\frac{1}{2} \wp\left(|\alpha|^{2}\right)|\alpha|^{-2}\left(\bar{\alpha} \nabla_{A} \alpha-\alpha \nabla_{A} \bar{\alpha}\right),
$$

this a connection on $E$ 's pullback over $\mathbb{R} \times M$. Here, and as previously, $\nabla_{A}$ denotes the covariant on $\mathbb{R} \times M$ as defined by $A$. The curvature, $F_{\hat{A}}$, of this connection is

$$
F_{\hat{A}}=(1-\wp) F_{A}-\wp^{\prime} \nabla_{A} \bar{\alpha} \wedge \nabla_{A} \alpha .
$$

If the assumptions of Lemma 3.6 hold, then

$$
\left|F_{\hat{A}}\right| \leq c_{0}\left(r^{-1}+r e^{-\sqrt{r} \operatorname{dist}\left(\cdot, X_{*}\right) / \kappa}\right)
$$

at all points with $s \in\left[s_{0}-R, s_{0}+R\right]$.

\section{3.d Behavior near $\mathbb{R} \times \gamma$ when $\gamma$ is elliptic}

Suppose that $\gamma \subset M$ is a Reeb orbit with a tubular neighborhood map of the sort described in (1-15). 
Lemma 3.11 There exists $\kappa \geq 1$ with the following significance: Suppose that $r \geq \kappa$, and that $\mathfrak{d}=(A, \psi=(\alpha, \beta))$ is an instanton solution to (1-11) with either $\mathrm{A}_{\mathfrak{d}}<r^{2}$ or $\mathrm{F}_{\mathfrak{d}} \geq-r^{2}$. Let $\gamma$ denote a Reeb orbit as just described. Then $|\beta| \leq \kappa r^{-1}$ and $\left|\nabla_{A} \beta\right| \leq \kappa r^{-1 / 2}$ at all points in $\mathbb{R} \times M$ with distance $\kappa^{-1}$ or less from $\mathbb{R} \times \gamma$.

By way of reminder, $\nabla_{A}$ here also refers to the covariant derivative defined on $\mathbb{R} \times M$ by viewing $A$ as a connection on the pullback of $E$ to $\mathbb{R} \times M$.

Proof of Lemma 3.11 The key point to note is that $J$ is integrable on a uniform radius tubular neighborhood of $\mathbb{R} \times \gamma$. Let $\mathbb{R} \times U$ denote this neighborhood.

With this last point in mind, note that the splitting $\mathbb{S}=E \oplus E K^{-1}$ identifies $\beta$ as an $E$-valued 2-form on $\mathbb{R} \times M$ which lies everywhere in the subbundle $T^{0,2}(\mathbb{R} \times M)$. Viewed in this way, the lower equation in (1-11) can be written as

$$
\bar{\partial}_{A} \alpha+\bar{\partial}_{A}^{\dagger} \beta=0 \text {. }
$$

Here, $\bar{\partial}_{A}^{\dagger}: C^{\infty}\left(\mathbb{R} \times M ; T^{0,2}(\mathbb{R} \times M) \otimes E\right) \rightarrow C^{\infty}\left(\mathbb{R} \times M ; T^{0,1}(\mathbb{R} \times M) \oplus E\right)$ is the formal $L^{2}$ adjoint of the composition of first exterior covariant differentiation and then projection to the $T^{0,2}$ summand. As $J$ is integrable, $\bar{\partial}^{2}=0$. As a consequence, (3-36) implies that

$$
\bar{\partial}_{A} \bar{\partial}_{A}^{\dagger} \beta+F_{A}^{0,2} \alpha=0
$$

on $\mathbb{R} \times U$. Here, $F_{A}^{0,2}$ denotes the $T^{0,2}(\mathbb{R} \times M)$ component of $A$ 's curvature 2-form. The Bochner-Weitzenboch formula for $\bar{\partial}_{A} \bar{\partial}_{A}^{\dagger}$ together with Lemma 3.1 and Lemma 3.6 can be used with (3-37) to see that $d^{\dagger} d|\beta|+2 r|\beta| \leq c_{0}$ on $\mathbb{R} \times U$. It follows as a consequence that $u=|\beta|-c_{0} r^{-1}$ obeys

$$
d^{\dagger} d u+2 r u \leq 0 \quad \text { on } \mathbb{R} \times U .
$$

Meanwhile, Lemma 3.1 finds $|u| \leq c_{0} r^{-1 / 2}$ on the boundary of $\mathbb{R} \times U$. This last observation, (3-38) and the fact that the Green's function for the operator $d^{\dagger} d+2 r$ obeys the bounds given by (3-30) implies that $|u| \leq c_{0} r^{-1 / 2} e^{-\sqrt{r} \operatorname{dist}(\cdot, R \times \partial U) / c_{0}}$. The bound $|\beta| \leq c_{0} r^{-1}$ follows from this.

To obtain the desired bound on $\left|\nabla_{A} \beta\right|$ at a given point $p$ in $\mathbb{R} \times U$, let $B$ denote the ball of radius $r^{-1 / 2}$ centered at $p$. Use parallel transport via $A$ and the Riemannian connection to view $\beta$ on $B$ as a section of the product bundle $B \times \mathbb{C}$. With this view understood, then (3-37) on $B$ can be written as

$$
d^{\dagger} d \beta+q_{0} \cdot d \beta=r q_{1} \beta+q_{2} \alpha,
$$


where the coefficients of $q_{0}$ and both $q_{1}$ and $q_{2}$ have absolute values bounded by $c_{0}$. Note also that $q_{0}$ is determined solely by the Riemannian metric, and its derivatives to a fixed order are bounded by $c_{0}$. Let $\chi^{p}$ denote the function with compact support on $B$ given by $\chi\left(r^{1 / 2} \operatorname{dist}(\cdot, p)\right)$. Let $g(\cdot, \cdot)$ denote the Green's function for the operator $\left(d^{\dagger} d+q_{0} \cdot d\right)$ on $B$ with Dirichlet boundary conditions. A standard asymptotic expansion finds

$$
|g|_{(p, x)} \mid \leq c_{0} \operatorname{dist}(p, x)^{-2} \text { and }|d g|_{(p, x)} \mid \leq c_{0} \operatorname{dist}(p, x)^{-3}
$$

at points with $\operatorname{dist}(p, x) \leq \frac{7}{8} r^{-1 / 2}$. Multiply both sides of (3-39) by $\chi^{p} g$, and then integrate the result. The result is an integral expression asserting

$$
\left.\beta\right|_{x}=\int_{B} g(x, \cdot) \mathfrak{q}
$$

where $q$ has support where $\operatorname{dist}(p, \cdot) \leq \frac{1}{2} r^{-1 / 2}$. Moreover, $|q|$ is bounded by $c_{0}$ because such is the case for both $r|\beta|$ and $|\alpha|$. Differentiate (3-41) now and use (3-40) with what was just said about $|q|$ to see that $\left|\nabla_{A} \beta\right| \leq c_{0} r^{-1 / 2}$.

\section{Instantons and pseudoholomorphic subvarieties: Part I}

This proposition below describes the fundamental relationship between instanton solutions to (1-11) and pseudoholomorphic subvarieties in $\mathbb{R} \times M$. Here as in Section 3, these equations are defined by a given pair $(a, J)$ and a given 1 -form $\mu \in \Omega$ with $\mathcal{P}$-norm bounded by 1 . The only unstated constraint is that $d a(\cdot, J(\cdot))$ is symmetric and positive definite.

Proposition 4.1 Given $\delta>0$ and $\mathcal{K} \geq l$, there exists $\kappa \geq 1$ with the following significance: Let $\mathbb{I}$ denote either $\mathbb{R}$ or a connected, open subset which can be bounded or not, but of total length at least $2 \delta^{-1}+16$. Suppose that $r \geq \kappa$, and that $\mathfrak{d}=(A, \psi=$ $(\alpha, \beta))$ is an instanton solution to (1-11) with either $\mathrm{A}_{\mathfrak{d}}<r^{2}$ or $\mathrm{F}_{\mathfrak{d}} \geq-r^{2}$, and with $\sup _{s \in I} \underline{\mathrm{M}}(s) \leq \mathcal{K}$. Let $I \subset \mathbb{I}$ denote a connected set of points with distance at least 7 from any boundary point of $\mathbb{I}$ and length $2 \delta^{-1}$.

- Each point in $I \times M$ where $|\alpha| \leq 1-\delta$ has distance $\kappa r^{-1 / 2}$ or less from a point where $\alpha$ is zero.

- There exists a finite set, $\vartheta$, whose components are pairs of the form $(C, m)$ where $C$ is a closed, irreducible pseudoholomorphic subvariety in a neighborhood of the closure of $I \times M$ and where $m$ is a positive integer. Moreover, no two pairs in $\vartheta$ share the same subvariety component. This set is such that 


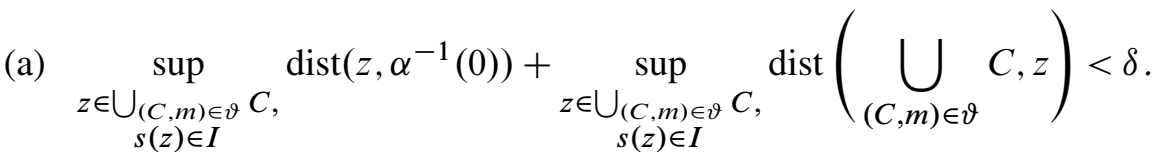

(b) Let $v$ denote the restriction to $I \times M$ of a 2 -form on $I \times M$ with $\|v\|_{\infty}=1$ and $\|\nabla v\|_{\infty} \leq \delta^{-1}$, and with support where $s \in I$. Define $\hat{A}$ from $A$ and $\alpha$ as in (3-33) using $\wp(x)=x$. Then

$$
\left|\frac{i}{2 \pi} \int_{I \times M} v \wedge F_{\hat{A}}-\sum_{(C, m) \in \vartheta} m \int_{C} v\right| \leq \delta .
$$

(c) $\sum_{(C, m) \in \vartheta} m \int_{C} d a \leq \kappa$.

Sections 4.a-4.c contain the proof of this proposition. The remaining subsections contain various related results. Note that the arguments in this section borrow much from Sections 4-6 from the article SW $\Rightarrow$ Gr in [6]. Note also that what is denoted in the rest of this section by $\widehat{A}$ is defined by (3-33) using $\wp(x)=x$.

\section{4.a Instantons at the length scale $r^{-1 / 2}$}

This subsection provides the instanton analog what is asserted in Proposition 4.2 of the article SW $\Rightarrow \mathrm{Gr}$ in [6]. This is the analog is stated as Lemma 4.3 in Part 2 of this subsection; Part 1 sets the stage.

Part 1 Introduce complex coordinates $\left(x_{1}, x_{2}\right)$ for $\mathbb{C}^{2}=\mathbb{R}^{4}$. Give $\mathbb{C}^{2}$ the standard metric with Kahler form $\omega_{0}=(i / 2)\left(d x_{1} \wedge d \bar{x}_{1}+d x_{2} \wedge d \bar{x}_{2}\right)$. Use $P^{+}: \wedge^{2} T^{*} \mathbb{C}^{2} \rightarrow$ $\wedge^{2} T^{*} \mathbb{C}^{2}$ to denote the projection onto the self dual subspace and $P^{-}$to denote the projection onto the anti-self dual subspace.

Of interest here are pairs $\left(A_{0}, \alpha_{0}\right)$ on $\mathbb{C}^{2}$ where $A_{0}$ is a unitary connection on the trivial bundle and $\alpha_{0}$ is a section of this bundle; and where:

- $\bar{\partial}_{A_{0}} \alpha_{0}=0$.

- $P^{+} F_{A_{0}}=-(i / 2)\left(1-\left|\alpha_{0}\right|^{2}\right) \omega_{0}$.

- $\left|\alpha_{0}\right| \leq 1$.

- $\left|P^{-} F_{A_{0}}\right| \leq\left|P^{+} F_{A_{0}}\right| \leq 2^{-1 / 2}\left(1-\left|\alpha_{0}\right|^{2}\right)$.

Proposition 4.1 in the article $\mathrm{SW} \Rightarrow$ Gr from [6] describes the pairs $\left(A_{0}, \alpha_{0}\right)$ that satisfy these conditions. The following proposition contains some of what is in Proposition 4.1 and some new things as well. 
Proposition 4.2 Suppose that $\left(A_{0}, \alpha_{0}\right)$ obeys (4-1).

- If $\left|\alpha_{0}\right|<1$ somewhere, then $\inf _{\mathbb{C}^{2}}\left|\alpha_{0}\right|=0$. If $\alpha_{0}^{-1}(0) \neq \varnothing$, then this locus is either all of $\mathbb{C}^{2}$ or a complex analytic subvariety of complex dimension 1.

- There exists $\kappa \geq 1$ that is independent of $\left(A_{0}, \alpha_{0}\right)$ and has the following significance: Let $X_{*} \subset \mathbb{C}^{2}$ denote the set of points where $1-\left|\alpha_{0}\right| \geq \kappa$. Then

$$
1-\left|\alpha_{0}\right|+\left|\nabla_{A_{0}} \alpha_{0}\right| \leq \kappa e^{-\operatorname{dist}\left(\cdot, X_{*}\right) / \kappa} .
$$

- If $\left|\alpha_{0}\right|<1$ somewhere, and if there exists $z \geq 1$ such that the integral of $\left(1-\left|\alpha_{0}\right|^{2}\right)$ over the ball of any given radius $R \geq 1$ centered at the origin is bounded by $z R^{2}$, then

(a) The locus $\alpha^{-1}(0)$ is a nonempty, complex algebraic subvariety with complex dimension 1. As such, this locus near any given point is the zero locus of a holomorphic polynomial.

(b) The order of the latter polynomial has an purely $z$-dependent upper bound.

(c) Given $z$, there exists $\kappa$ such that $1-\left|\alpha_{0}\right|+\left|\nabla_{A_{0}} \alpha_{0}\right| \leq \kappa e^{-\operatorname{dist}\left(\cdot, \alpha^{-1}(0)\right) / \kappa}$.

If, in addition, the integral over $\mathbb{C}^{2}$ of $\left|P^{+} F_{A_{0}}\right|^{2}-\left|P^{-} F_{A_{0}}\right|^{2}$ is finite, then

(d) This integral is a nonnegative integer multiple of $4 \pi^{2}$.

(e) If the latter integral is zero, then $\left(A_{0}, \alpha_{0}\right)$ is the pullback via a projection $\mathbb{C}^{2} \rightarrow \mathbb{C}$ of a solution on $\mathbb{C}$ to the vortex equations in (1-4) and $\alpha_{0}^{-1}(0)$ is a union of planes.

- The set of gauge equivalence classes of pairs $\left(A_{0}, \alpha_{0}\right)$ that obey (4-1) is sequentially compact with respect to convergence on compact subsets of $\mathbb{C}^{2}$ in the $C^{\infty}$ topology.

Proof of Proposition 4.2 The only assertions not contained in Proposition 4.1 of the article SW $\Rightarrow$ Gr from [6] are the assertion in the first item that the infimum of $\left|\alpha_{0}\right|$ is zero if $\left|\alpha_{0}\right|<1$ at any point and the bound given in the second item. To prove that $\inf _{\mathbb{C}^{2}}\left|\alpha_{0}\right|=0$, suppose that $\alpha_{0}$ is nowhere zero. If so, there is a gauge transformation that writes $\alpha_{0}=e^{u}$ with $u$ a real valued function. The first item in (4-1) is satisfied if and only if $A_{0}=-\bar{\partial} u+\partial u$. The second item is obeyed if and only if

$$
d^{\dagger} d u=\left(1-e^{2 u}\right)
$$

Here, $d$ denotes the exterior derivative on $\mathbb{R}^{4}$ and $d^{\dagger}$ denotes its formal $L^{2}$ adjoint. The condition in the third point of (4-1) requires that $u<0$ at some point, and so the maximum principle has $u<0$ everywhere. If $u$ is bounded from below, then there is a 
uniform bound on $|\nabla u|$. Indeed, if $x \in \mathbb{C}^{2}$, then (4-2) implies that

$$
\begin{array}{r}
u(x)=\frac{1}{2 \pi^{2}} \int_{\mathbb{C}^{2}}\left(\frac{1}{|x-(\cdot)|^{2}}\left(1-e^{2 u}\right)-u \frac{1}{|x-(\cdot)|^{2}} d^{\dagger} d \chi_{x}\right. \\
\left.+2 u\left(d \frac{1}{|x-(\cdot)|^{2}}, d \chi_{x}\right\rangle\right),
\end{array}
$$

where $\chi_{x}(\cdot)=\chi(|x-(\cdot)|)$ and where $\langle$,$\rangle denotes Euclidean inner product. Dif-$ ferentiate (4-3) to obtain the uniform bound on $\nabla u$. Meanwhile, differentiating (4-2) finds that

$$
d^{\dagger} d|\nabla u|+e^{2 u}|\nabla u| \leq 0 .
$$

Given that $u$ is bounded from below, there exists $c>0$ such that $d^{\dagger} d|\nabla u|+c|\nabla u| \leq 0$. This last inequality can not be satisfied with $|\nabla u|$ uniformly bounded unless it is everywhere zero. Indeed, if $R \geq 1$, then $|\nabla u|$ on the ball of radius $R$ must be less than the solution in this ball to the equation $d^{\dagger} d g_{R}+c g_{R}=0$ with boundary condition equal to the supremum of $|\nabla u|$. This solution at the origin is bounded $c_{0} R^{-2} e^{-\sqrt{c} R} \sup _{\mathbb{C}^{2}}|\nabla u|$. This last expression converges to zero as $\mathbb{R} \rightarrow \infty$.

The proof of the second item can be proved using arguments that are very much like those used to prove Lemma 3.8. In fact, the algebraic manipulations in this case are simple as there are no Riemannian curvature terms to deal with. The details are straightforward and so omitted.

Part 2 Suppose that $x \in \mathbb{R} \times M$ and that $(A, \psi=(\alpha, \beta))$ is an instanton solution to (1-11). Fix complex coordinates centered at $x$ of the following sort: First, the coordinates are Gaussian normal ones. In addition, there is an identification of $\mathbb{R}^{4}$ with $\mathbb{C}^{2}$ that writes the resulting complex coordinate functions as $\left(y_{1}, y_{2}\right)$; and these are such that $\left\{d y_{1}, d y_{2}\right\}$ span $\left.T^{1,0}(\mathbb{R} \times M)\right|_{x}$. Let $\rho>c_{0}^{-1}$ be such that these coordinates are defined on the ball of radius $\rho$ in $\mathbb{C}^{2}$. Use these coordinates to identify this ball with its image in $\mathbb{R} \times M$. Define the map $\hat{r}: \mathbb{C}^{2} \rightarrow \mathbb{C}^{2}$ using the rule $\hat{r}(y)=r^{-1 / 2} y$. The pullback of $(A, \alpha)$ via $\hat{r}$ to the ball of radius $r^{1 / 2} \rho$ in $\mathbb{C}^{2}$ is denoted in what follows by $\left(A_{x}, \alpha_{x}\right)$.

Lemma 4.3 Given $z \geq 1, R \geq 1, k \in\{0,1, \ldots\}$ and $\varepsilon>0$, there exists $\kappa>1$ with the following significance: Suppose that $r \geq \kappa$ and that $(A, \psi)$ is an instanton solution to (1-11). Let $x \in \mathbb{R} \times M$ and suppose that $\left|(\partial / \partial s) A-B_{A}\right| \leq r\left(1-|\alpha|^{2}\right)+z$ on the ball of radius 1 centered at $x$. Under these conditions, there exists a solution $\left(A_{0}, \alpha_{0}\right)$ to (4-1) on $\mathbb{C}^{2}$ such that $\left(A_{x}, \alpha_{x}\right)=\left(A_{0}+\hat{a}, \alpha_{0}+\eta\right)$ where $(\hat{a}, \eta)$ has $C^{k}$ norm less than $\varepsilon$ on the ball of radius $R$ in $\mathbb{C}^{2}$. 
Proof of Lemma 4.3 The proof is essentially the same as that of Proposition 4.2 in the article $\mathrm{SW} \Rightarrow \mathrm{Gr}$ from [6].

\section{4.b Proof of bullet 1 of Proposition 4.1}

Suppose that the assertion is false. If so, there exists $\delta>0$ and $\mathcal{K} \geq 1$, and for each $n \in\{1,2, \ldots\}$, a set $\left\{\left(r_{n},\left(A_{n}, \psi_{n}\right)\right), x_{n}\right\}$ such that the assumptions of Proposition 4.1 hold for the interval $\mathbb{I}=[-8,8]$, and such that $\left|\alpha_{n}\right| \leq 1-\delta$ at a point $x_{n} \in[-1,1] \times M$, and with $\operatorname{dist}\left(x_{n}, \alpha_{n}^{-1}(0)\right)>n r^{-1 / 2}$. No generality is lost by assuming that what is said by Lemma 4.3 applies to $\left(A_{n}, \psi_{n}\right)$ using for $x$ the point $x_{n}$ with the constant $\varepsilon<n^{-1} \delta$. Let $\left\{\left(A_{0 n}, \alpha_{0 n}\right)\right\}_{n=1,2, \ldots}$ denote the corresponding sequence of solutions to (4-1). Use the final item in Proposition 4.2 to find gauge transformations that yield a new sequence with a convergent subsequence. Let $\left(A_{0}, \alpha_{0}\right)$ denote the limit. Note in particular that $\left|\alpha_{0}\right|$ is no greater than $1-\delta$ at the origin. For each $R \geq 1$, write the integral of $\left(1-\left|\alpha_{0}\right|^{2}\right)$ over the radius $R$ ball in $\mathbb{C}^{2}$ as $\mathcal{K}_{R} R^{2}$.

Suppose that $\left\{\mathcal{K}_{R}\right\}_{R \geq 1}$ is bounded. Then Point (a) of the third item in Proposition 4.2 implies that there is nonconstant, holomorphic polynomial $h$ on $\mathbb{C}^{2}$ and a smooth function $u$ such that $\alpha_{0}=e^{u} h$. Thus, $\alpha_{0}^{-1}(0)=h^{-1}(0)$. Let $D \subset \mathbb{C}^{2}$ denote an embedded, closed holomorphic disk of radius 1 or less that intersects $\alpha_{0}^{-1}(0)$ at its center and has boundary disjoint from $\alpha^{-1}(0)$. The winding number of $\alpha_{0}(0) /\left|\alpha_{0}(0)\right|$ on the boundary of $D$ is thus positive. The form of convergence that is asserted by Proposition 4.2 and the final item of Proposition 4.2 implies that each large $n$ version of $\alpha_{n x_{n}}$ is nonzero on the boundary of $D$ and also has positive winding number. As a consequence, there is a zero of each such section in $D$. Let $d$ denote the distance between $d$ 's center and the origin in $\mathbb{C}^{2}$. Then $\alpha_{n}$ has a zero with distance less than $(d+1) r^{-1 / 2}$ from $x_{n}$ when $n$ is large. This contradicts the assumptions. Thus, the sequence $\left\{\mathcal{K}_{R}\right\}_{R \geq 1}$ must be unbounded.

Suppose that the sequence $\left\{\mathcal{K}_{R}\right\}_{R \geq 1}$ is unbounded. Given the second item in Proposition 4.2, the convergence asserted by Proposition 4.2 has the following additional consequence: Fix $R \geq 1$, and then the integral of any sufficiently large $n$ version of the function $r\left(1-\left|\alpha_{n}\right|^{2}\right)$ over the ball of radius $R r^{-1 / 2}$ centered at $x_{n}$ will be greater than $\frac{1}{2} \mathcal{K}_{R} R^{2} r^{-1}$. This runs afoul of the second bullet of Lemma 3.10 when $n$ is large.

Thus, there is no sequence of the sort that would arise were the first item of Proposition 4.1 false. 


\section{4.c Convergence}

This subsection addresses the second bullet of Proposition 4.1. The discussion that follows has four parts. The fourth part contains the proof of the second bullet of Proposition 4.1.

Part 1 Suppose that $\left\{\left(r_{n},\left(A_{n}, \psi_{n}\right)\right)\right\}_{n=1,2, \ldots}$ is a sequence of the following sort: First, the sequence $\left\{r_{n}\right\}_{n=1,2, \ldots} \subset[1, \infty)$ is increasing and unbounded. Second, any given $\left(A_{n}, \psi_{n}\right)$ is an instanton solution to the $r=r_{n}$ version of (1-11) that obeys the assumptions of Proposition 4.1 for $I=[-8,8]$ and for a given $\mathcal{K} \geq 1$. A verbatim repeat of what is said in Sections $5 \mathrm{~b}-5 \mathrm{c}$ of $\mathrm{SW} \Rightarrow$ Gr from [6] finds a subsequence of $\left\{\left(r_{n},\left(A_{n}, \psi_{n}\right)\right)\right\}_{n=1,2, \ldots}$ now renumbered consecutively from 1 , a closed set $\Sigma \subset[-3,3] \times M$ and a bounded linear functional, $m$ on the space $C^{0}\left([-3,3] \times M ; \bigwedge^{2} T^{*}([-3,3] \times M)\right)$ with the properties listed below:

Property 1 The set $\Sigma$ has finite, 2-dimensional Hausdorff measure. Let $\Sigma_{0}$ denote the part of $\Sigma$ in $[-2,2] \times M$. If $x \in \Sigma_{0}$, then the Hausdorff measure of $\Sigma$ 's intersection with the ball of radius $\rho$ centered at $x$ lies between $c_{0}^{-1} \rho^{-2}$ and $c_{0} \rho^{2}$.

Property 2 The distribution $m$ has support on $\Sigma$. Moreover, $m$ is also closed in the sense that $m(d \sigma)=0$ if $\sigma$ is a 1 -form with compact support in $[-2,2] \times M$. Finally, $m$ annihilates sections of $\bigwedge^{0,2} T^{*}([-2,2] \times M)$; and it is positive on the forms

$$
\left(d s \wedge a+\frac{1}{2} * a\right)+v
$$

if $v$ is anti-self dual with $|v| \leq 2^{1 / 2}$.

Property 3 Let $D \subset \mathbb{C}$ denote the open unit disk and $\underline{D}$ denote its closure. Suppose that $\phi$ is an orientation preserving embedding of $\underline{D} \times \underline{D} \rightarrow(-2,2) \times M$. Assume in addition that $\phi(\underline{D} \times \partial \underline{D})$ is disjoint from $\Sigma_{0}$. Let $v$ denote a smooth 2 -form on $\phi(\underline{D} \times \underline{D})$ that vanishes near $\phi(\partial \underline{D} \times \underline{D})$ and integrates to 1 on any disk of the form $\phi(\underline{D}, z)$ with $z \in \underline{D}$. Then $m(v)$ is an integer. Moreover, $m(v) \geq 1$ if $\phi(0, \cdot)$ intersects $\Sigma_{0}$ and maps $D$ in an orientation preserving fashion onto a pseudoholomorphic disk.

Property 4 The following sequences converge with limit zero: To describe the first sequence, let $\Sigma_{0}=\Sigma \cap([-2,2] \times M)$. Here is the $n$-th element of the first sequence:

$$
\sup _{z \in \Sigma_{0}} \operatorname{dist}\left(z, \alpha_{n}^{-1}(0)\right)+\sup _{z \in \alpha_{n}^{-1}(0)} \operatorname{dist}\left(\Sigma_{0}, z\right) .
$$


Each of the remaining sequences is labeled by a $2-$ form on $[-2,2] \times M$ with supremum norm equal to 1 and compact support. Let $v$ denote such a form. The sequence labeled by $v$ has $n$-th element

$$
\frac{i}{2 \pi} \int_{[-2,2] \times M} v \wedge F_{\hat{A}_{n}}-m(v) .
$$

To say something about the proof that these properties hold, remark that the assumptions on the sequence imply that Lemmas 3.1, 3.6, 3.8, 3.9 and 3.10 can be invoked on $[-4,4] \times M$. These lemma with Lemma 3.1 are the key inputs to the arguments used to prove Lemmas 5.1 and 5.2 in the article $\mathrm{SW} \Rightarrow$ Gr from [6]. Indeed, Lemma 3.9 implies the following: Let $\left\{\left(A_{n}, \psi_{n}\right)\right\}_{n=1,2, \ldots}$ denote the original sequence. Then the sequence of distributions

$$
\left\{v \rightarrow(i /(2 \pi)) \int_{[-2,2] \times M} v \wedge F_{A_{n}}\right\}_{n=1,2, \ldots}
$$

on $C^{0}\left([-4,4] \times M ; \bigwedge^{2} T^{*}([-4,4] \times M)\right)$ is bounded. Granted this, then this sequence of distributions has a weakly convergent subsequence. A convergent subsequence is chosen and the limit distribution is taken to be $m$. The conclusions of Lemma 3.10 are used to construct a refined subsequence whose version of the set $\Sigma_{0}$ is the support of $m$. The fact that $m$ is closed is a consequence of the fact that $(i /(2 \pi)) F_{\hat{A}_{n}}$ is a closed 2-form. The remaining assertions of Property 2 follow in a straightforward fashion using (3-34) and (3-35) and the bounds given by Lemmas 3.1, 3.6, 3.8 and 3.9. The assertion made in Property 3 about the integer value of $m$ follows using two facts: First, $(i /(2 \pi)) F_{\widehat{A}_{n}}$ represents the first Chern class of a complex line bundle $E$. Second, this 2 -form is nearly zero at any given point in the complement of $\Sigma_{0}$ when $n$ is large. The positivity of $m(v)$ when $\phi$ maps $0 \times D$ onto a pseudoholomorphic disk follows using Lemma 4.3.

Part 2 The notion of a positive cohomology assignment is introduced in Section 6a of the article SW $\Rightarrow$ Gr from [6]. The definition refers to the notion of an admissible map. The analogous definition in the present context requires first the specification of a closed subset $\Sigma_{0} \subset[-2,2] \times M$ with finite 2-dimensional Hausdorff measure. With the latter specified, a smooth map $\sigma: D \rightarrow(-1,1) \times M$ is said to be admissible if it extends as a continuous map to $\partial \underline{D}$ and maps the latter to the complement in $(-1,1) \times M$ of $\Sigma_{0}$ 's intersection with $(-1,1) \times M$. The set of admissible maps is open in $C^{\infty}(D ;(-1,1) \times M)$. A homotopy $h:[0,1] \times D \rightarrow(-1,1) \times M$ is admissible if it extends as a continuous map from $[0,1] \times \partial \underline{D}$ into $(-1,1) \times M$ and is such that $h(t, \cdot)$ is admissible for each $t \in[0,1]$. A positive cohomology assignment associates 
to each admissible map $\sigma$ and integer, $I(\sigma)$, subject to the following rules:

- $I(\sigma)=0$ if $\sigma(D)$ is disjoint from $\Sigma_{0}$.

- If $\sigma_{0}$ and $\sigma_{1}$ are admissable maps that are homotopic via an admissable homotopy, then $I\left(\sigma_{0}\right)=I\left(\sigma_{1}\right)$.

- If $\sigma$ is admissable and if $\phi: D \rightarrow D$ is a proper, degree $k$ map, then $I(\sigma \circ \phi)=k I(\sigma)$.

- Suppose that $\sigma$ is admissable and that $\sigma^{-1}\left(\Sigma_{0}\right)$ is contained in a disjoint union $\bigcup_{v} D_{v} \subset D$ where each $D_{v}$ is the image via an orientation preserving embedding, $\theta_{v}$, of $D$ into $D$. Then $I(\sigma)=\sum_{v} I\left(\sigma \circ \theta_{v}\right)$.

- If $\sigma$ is an orientation preserving, pseudoholomorphic embedding whose image intersects $\Sigma$, then $I(\sigma)>0$.

As is explained next, the distribution $\mathfrak{m}$ from Part 1 can be used to define a positive cohomology assignment for Part 1's set $\Sigma_{0}$. This is done as follows: Suppose first that $\sigma$ is an admissible embedding that extends as a smooth embedding of $\underline{D}$. Then $\sigma$ extends as an embedding $\phi: \underline{D} \times \underline{D} \rightarrow(-1,1) \times M$ such that $\phi(0, \cdot)=\sigma$. Fix a 2-form $a$ with compact support on $\underline{D}$ and with integral 1 and pull the latter back to $\underline{D} \times \underline{D}$ via the projection to the first factor. Push this form forward by $\phi$. Define $I(\sigma)=m(v)$. The fact that $m$ is closed implies that $I(\sigma)$ has no dependence on either the form $v$ or the extension $\phi$ of $\sigma$. The definition just given can be used to define $I(\sigma)$ in the case when $\sigma$ immerses $D$ and is such that $\sigma^{-1}\left(\Sigma_{0}\right)$ is contained in a disjoint union of subdisks on which $\sigma$ is $1-1$. Indeed, define $I(\cdot)$ as above for each such subdisk and take $I(\sigma)$ to be the sum of the resulting numbers. Granted the preceding, suppose now that $\sigma$ is any given admissible map. A very small perturbation, $\sigma_{0}$, of $\sigma$ will be an admissible immersion of the sort just described. Define $I(\sigma)$ to equal $I\left(\sigma_{0}\right)$. Since $m$ is closed, the value of $I(\sigma)$ does not depend on $\sigma_{0}$ if $\sup _{z \in D} \operatorname{dist}\left(\sigma(z), \sigma_{0}(z)\right)$ is sufficiently small. The first four points in (4-8) follow either directly from the definition, or from the fact that $m$ is closed. The fact that $I(\cdot)$ is an integer follows from Property 3 in Part 1 above, as does the fact that $I(\sigma)$ is positive when $\sigma^{-1}(\Sigma) \neq \varnothing$ and $\sigma$ maps $D$ onto a pseudoholomorphic disk.

Part 3 Let $\vartheta$ denote a finite set whose typical element is a pair $(C, m)$ of the following sort: First, $C$ is the restriction to $[-1,1] \times M$ of a closed, irreducible, oriented 2dimensional subvariety of some open neighborhood in $\mathbb{R} \times M$ of $[-1,1] \times M$ with nonempty intersection with $(-1,1) \times M$. Second, $m$ is a positive integer. Assume, in addition that no two pairs from $\vartheta$ share the same subvariety.

Suppose that $\bigcup_{(C, m) \in \vartheta} C=\Sigma_{0}$. Each admissible map has a well defined intersection number with each subvariety from $\vartheta$. If $\sigma$ is an admissible map, and $(C, m) \in \vartheta$, 
use $I_{C}(\sigma)$ to denote the intersection number of $\sigma$ with $C$. Define $I(\sigma)$ to equal $\sum_{(C, m) \in \Theta} m I_{C}(\sigma)$. The latter obeys the top four positive cohomology assignment axioms in (4-8). It obeys the final axiom if and only if each subvariety from $\vartheta$ is pseudoholomorphic. A positive cohomology assignment of this sort is said to be carried by $\vartheta$.

The next lemma asserts that a positive cohomology assignment for $\Sigma_{0}$ is necessarily of the sort just described. This is the analog here of Proposition 6.1 in the article $\mathrm{SW} \Rightarrow \mathrm{Gr}$ from [6].

Lemma 4.4 Let $I(\cdot)$ denote a positive cohomology assignment as described in Part 2. This positive cohomology assignment is carried by a finite set $\vartheta$ whose typical element is a pair $(C, m)$ where $C$ is a closed, irreducible, pseudoholomorphic subvariety in a neighborhood of $[-1,1] \times M$ and where $m$ is a positive integer.

Proof of Lemma 4.4 The proof of Proposition 6.1 in the article SW $\Rightarrow$ Gr from [6] is essentially local in nature, and so can be exported for use in the present context. To elaborate on this, agree first to extend the definition of an admissible map so as to allow maps from $D$ into $(-2,2) \times M$. Thus, a map in this context is admissible if it extends to $\partial \underline{D}$ so as to map the latter to the complement of $\Sigma_{0}$ in $(-2,2) \times M$. Mimic what is done in Part 2 above to define a positive cohomology assignment on the set of admissible maps from $D$ into $(-2,2) \times M$. This done, the arguments in Sections $6 \mathrm{~b}-6 \mathrm{e}$ of the article $\mathrm{SW} \Rightarrow \mathrm{Gr}$ from [6] can be applied to prove the lemma. Note that the proof establishes that the subvarieties from the pairs in $\vartheta$ live in $(-2,2) \times M$.

Part 4 This part contains the following.

Proof of bullet 2 of Proposition 4.1 Suppose $\mathbb{I}, \delta, \mathcal{K}$ and $I$ are such that the proposition were false. There would then be a sequence $\left\{\left(r_{n},\left(A_{n}, \psi_{n}=\left(\alpha_{n}, \beta_{n}\right)\right)\right)\right\}_{n=1,2, \ldots}$ where $\left\{r_{n}\right\}_{n=1,2, \ldots}$ is a sequence in $[1, \infty)$ that is increasing without bound and where any given $\left(A_{n}, \psi_{n}\right)$ is an instanton solution to (1-11) that obeys the assumptions of the proposition but not the conclusions.

What follows can be arranged by passing to a diagonal subsequence of the original sequence $\left\{\left(r_{n},\left(A_{n}, \psi_{n}\right)\right)\right\}_{n=1,2, \ldots}$ and then renumbering this subsequence consecutively from 1 . Fix $s_{0} \in \frac{1}{2} Z \cap I$. Then translate each $\left(A_{n}, \psi_{n}\right)$ along the $\mathbb{R}$ factor of $\mathbb{R} \times M$ by $-s_{0}$ and apply the arguments in Part 1 to the result. The result is a set $\Sigma=\Sigma_{s_{0}} \subset[-2,2] \times M$, its subset $\sum_{0 s_{0}} \subset[-1,1] \times M$ and a distribution $m_{s_{0}}$; these obeying Properties 1-4 of Part 1. As noted in Part 2 of Section 4.c, they define a positive cohomology assignment. According to Lemma 4.4, this positive cohomology assignment is carried by a set $\vartheta=\vartheta_{s_{0}}$ of the sort that is described at the outset of 
Part 3. Translate the subvariety components of this set along the $\mathbb{R}$ factor of $\mathbb{R} \times M$ so that the origin goes to $s_{0}$ and so that all subvariety components from the pairs that comprise $\vartheta_{s_{0}}$ sit in a neighborhood of $\left[s_{0}-1, s_{0}+1\right] \times M$.

Let $\vartheta_{s_{0}}^{+}$denote the subset of pairs in $\vartheta_{s_{0}}$ whose subvariety component has nonempty intersection with $[0,1] \times M$, and let $\vartheta_{s_{0}}^{-} \subset \vartheta_{s_{0}}$ denote the subset of pairs whose subvariety component has nonempty intersection with $[-1,0] \times M$. The convergence of the sequences that are described in Property 4 of Part 1 imply that the $s \rightarrow s_{0}$ translate of $\vartheta_{s_{0}}^{+}$must agree with the $s \rightarrow s_{0}+\frac{1}{2}$ translate of $\vartheta_{s_{0}+1 / 2}^{-}$. This is to say that the subvarieties and associated integers match up. As a consequence, there exists a set, $\vartheta$, of pairs whose typical element has the form $(C, m)$ with $C$ a closed, irreducible, pseudoholomorphic subvariety of a neighborhood $I \times M$ in $\mathbb{R} \times M$, and with $m$ a positive integer. Note that this set $\vartheta$ must be finite because Property 4 in Part 1 implies an $s_{0^{-}}$ independent bound on both the integer component of any pair from $\vartheta_{s_{0}}$, and the area of the intersection with $(-1,1) \times M$ of the subvariety component of any pair from $\vartheta_{s_{0}}$.

It follows from the manner of convergence that is described in Property 4 of Part 1 that

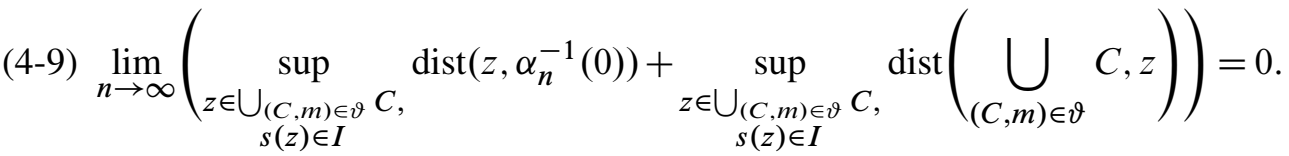

In particular, Assertion (a) of the second item of Proposition 4.1 holds if $n$ is sufficiently large. To obtain Assertion (b) of the second item, note that there exists a finite set, $\Omega_{\delta}$, of compactly supported 2-forms on $\mathbb{R} \times M$ such that any 2-form $v$ where $s \in I$ and with both $\|v\|_{\infty} \leq 1$ and $\|\nabla v\|_{\infty} \leq \delta^{-1}$ has distance $\frac{1}{8} \delta$ in the $C^{0}$ metric topology from some form in $\Omega_{\delta}$. Given that $\Omega_{\delta}$ is a finite set, (4-7) implies that

$$
\left|\frac{i}{2 \pi} \int_{I \times M} v \wedge F_{\hat{A}_{n}}-\sum_{(C, m) \in \vartheta} m \int_{\mathbb{C}} v\right| \leq \frac{1}{8} \delta
$$

for all $v \in \Omega_{\delta}$ when $n$ is large. Thus, Assertion (b) of the second item in Proposition 4.1 also holds when $n$ is large.

To obtain Assertion (c), note first that the form $d a$ is nonnegative on the tangent planes to any pseudoholomorphic subvariety. This understood, if $(C, m) \in \vartheta$, then the integral of $d a$ over $\mathbb{C}$ is finite if there is a $\delta$-independent upper bound to its integral over the $|s| \leq$ $\delta^{-1}$ part of $C$. Such a finite upper bound follows using the Assertion (b) of the second item with $v=\chi\left(|s|-\delta^{-1}+1\right) d a$. Note in particular that the integral of the wedge of this form with $F_{\hat{A}_{n}}$ can be bounded by an $n$-independent multiple of $\mathcal{K}$ by first integrating by parts and then applying (4-10) and Lemma 3.9 to the resulting expression. 


\section{4.d Multiple limits}

Fix $\delta>0, \mathcal{K} \geq 1$ and an unbounded set $\mathbb{I} \subset \mathbb{R}$ for use in Proposition 4.1. Fix $r \gg 1$ and an instanton solution, $\mathfrak{d}=(A, \psi=(\alpha, \beta))$, to (1-11) that obeys the hypothesis of Proposition 4.1. As will now be explained, the instanton $\mathfrak{d}$ can well determine via Proposition 4.1 a number of very distinct sets of the sort denoted in Proposition 4.1 by $\vartheta$. To say more, first translate $\mathfrak{d}$ along the $\mathbb{R}$ factor of $\mathbb{R} \times M$ so that the following is true: There exist an unbounded set of consecutive integers, $\Lambda$, such that $0 \in \Lambda$ and such that if $k \in \Lambda$, then $\mathbb{I}_{k}=\left[-\delta^{-1}-8+\frac{1}{2} k \delta^{-1}, \delta^{-1}+8+\frac{1}{2} k \delta^{-1}\right]$ is in $\mathbb{I}$ and the union, $\bigcup_{k \in \Lambda} \mathbb{I}_{k}$, is all of $I$. There exists such a translation by virtue of the fact that $\mathbb{I}$ is noncompact. Proposition 4.1 supplies a set $\vartheta=\vartheta_{k}$ for each interval $I_{k}$. Note that each subvariety from a pair in any given $\vartheta_{k}$ is defined on the whole of $\mathbb{R} \times M$. Even so, the points in $\alpha^{-1}(0)$ are guaranteed to have distance $\delta$ or less from $\Sigma_{k}=\bigcup_{(C, m) \in \vartheta_{k}} C$ only if $s \in I_{k}=\left[-\delta^{-1}+\frac{1}{2} k \delta^{-1}, \delta^{-1}+\frac{1}{2} k \delta^{-1}\right]$. Of course, the points in $\Sigma_{k}$ that sit where $s \in I_{k} \cap I_{k+1}$ have distance $\delta$ or less from those in $\Sigma_{k+1}$, and vice-versa; but there is no apriori guarantee that such is the case for all $s \in \mathbb{R}$. If such were the case for all $s \in \mathbb{R}$, then one could see about taking $\vartheta_{k}=\vartheta_{k+1}$. This then begs the following question:

Can the collection $\left\{\vartheta_{k}\right\}_{k \in \Lambda}$ from Proposition 4.1 be defined so as to have a finite number of elements? If so, can such a set be chosen whose size is apriori bounded in a $\delta$-independent fashion?

The next proposition asserts that the set $\left\{\vartheta_{k}\right\}_{k \in \Lambda}$ can be chosen so as to have a finite number of distinct elements; a number with bound determined solely by $\mathcal{K}$.

Proposition 4.5 Given $\mathcal{K} \geq 1$, suppose that each Reeb orbit with length at most $(1 /(2 \pi)) \mathcal{K}$ is nondegenerate. There exists $\kappa \geq 1$, and given $\delta>0$, there exists $\kappa_{\delta} \geq 1$ which, with $\kappa$, has the following significance: Suppose that $r \geq \kappa_{\delta}$, and that $\mathfrak{d}=(A, \psi=(\alpha, \beta))$ is an instanton solution to (1-11) with either $\mathrm{A}_{\mathfrak{d}}<r^{2}$ or $\mathrm{F}_{\mathfrak{d}} \geq-r^{2}$. Let $\mathbb{I} \subset \mathbb{R}$ denote a connected subset of length at least $2 \delta^{-1}+16$ such that $\sup _{s \in \mathbb{I}} \underline{\mathrm{M}}(s) \leq \mathcal{K}$. Let $I \subset \mathbb{I}$ denote the set of points with distance at least 7 from any boundary point of $\mathbb{I}$. Then

- Each point in $I \times M$ here $|\alpha| \leq 1-\delta$ has distance $\kappa r^{-1 / 2}$ or less from a point where $\alpha$ is zero.

- There exists

(a) A positive integer $N \leq \kappa$ and a cover of $I$ as $\bigcup_{1 \leq k \leq N} I_{k}$ by connected open sets of length at least $2 \delta^{-1}$. These are such that $I_{k} \cap I_{k^{\prime}}=\varnothing$ if $\left|k-k^{\prime}\right|>1$. In addition, if $\left|k-k^{\prime}\right|=1$, then $I_{k} \cap I_{k^{\prime}}$ has length between $\frac{1}{128} \delta^{-1}$ and $\frac{1}{64} \delta^{-1}$. 
(b) For each $k \in\{1,2, \ldots, N\}$, a set $\vartheta_{k}$ whose typical element is a pair $(C, m)$ where $m$ is a positive integer and where $C \subset \mathbb{I} \times M$ is a pseudoholomorphic subvariety defined on a neighborhood of $I_{k} \times M$. These elements of $\vartheta_{k}$ are constrained so that no two pair share the same subvariety component; and so that

$$
\sum_{(C, m) \in \vartheta_{k}} m \int_{C} d a<\kappa
$$

In addition, the collection $\left\{\vartheta_{k}\right\}_{k=1, \ldots, N}$ is such that

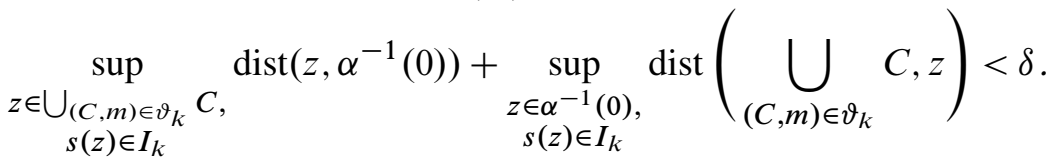

(2) Let $k \in\{1, \ldots, N\}$, let $I^{\prime} \subset I_{k}$ denote an interval of length 1 , and let $v$ denote the restriction to $I^{\prime} \times M$ of a $2-$ form on $\mathbb{I} \times M$ with $\|v\|_{\infty}=1$ and $\|\nabla v\|_{\infty} \leq \delta^{-1}$. Then

$$
\left|\frac{i}{2 \pi} \int_{I^{\prime} \times M} v \wedge F_{\hat{A}}-\sum_{(C, m) \in \vartheta} m \int_{C} v\right| \leq \delta .
$$

- Suppose that $\mathbb{I}$ is unbounded from above. Fix $\mathrm{E}_{+} \leq \mathcal{K}$ and assume with regards to the Reeb orbits only that all with length at most $(1 /(2 \pi)) \mathrm{E}_{+}$are nondegenerate. Assume in addition that $\lim _{s \rightarrow \infty} \mathrm{E}\left(\left.\mathfrak{d}\right|_{s}\right) \leq \mathrm{E}_{+}$. Then the preceding conclusions hold with the constant $\kappa$ depending on $\mathcal{K}$ and $\mathrm{E}_{+}$, and with $\kappa_{\delta}$ depending on the latter and on $\delta$. Moreover, if $\mathbb{I}=\mathbb{R}$ and all Reeb orbit of length at most $(1 /(2 \pi)) \mathrm{E}_{+}$are nondegenerate, then $\lim _{s \rightarrow-\infty} \mathrm{E}\left(\left.\mathfrak{d}\right|_{s}\right) \leq \mathrm{E}_{+}+\delta$.

Proof of Proposition 4.5 The argument for the first bullet is the same as that given for the analogous point of Proposition 4.1. The proof of second bullet has four parts. Part 5 of what follows proves the third bullet.

Part 1 This part and Part 2 of the proof supply conditions that force a pseudoholomorphic subvariety to lie everywhere close to a Reeb orbit. The following lemma refers to a constant, $\ell_{M}$, which is the smallest of the lengths of the Reeb orbits.

Lemma 4.6 Given $\mathcal{N} \geq 1$ and $\varepsilon>0$, there exists $\kappa_{\varepsilon}>1$ with the following significance: Suppose $C$ is a closed, irreducible, pseudoholomorphic subvariety in a neighborhood of $[-4,4] \times M$ such that both $\int_{\mathbb{C}} d a<\kappa_{\varepsilon}^{-1}$ and $\int_{C \cap([-3,3] \times M)} d s \wedge a \leq \mathcal{N}$. Then each point of $\left.C\right|_{s}$ for $|s| \leq 1$ has distance along $M$ no greater than $\varepsilon$ from some Reeb orbit, $\gamma$, of length less than $\mathcal{N}+\varepsilon$. Moreover, there is a positive integer $m \leq \ell_{M}^{-1} \mathcal{N}$ 
such that if $v$ is a smooth $2-$ form on $[-1,1] \times M$ with $\|v\|_{\infty}=1$ and $\|\nabla v\|_{\infty} \leq \varepsilon^{-1}$, then

$$
\left|\int_{C \cap([-1,1] \times M)} v-m \int_{[-1,1] \times \gamma} v\right| \leq \varepsilon .
$$

Proof of Lemma 4.6 This is proved by invoking Lemma 4.4 as follows: Suppose that there is no such $\kappa_{\varepsilon}$. There would then exist a sequence $\left\{C_{k}\right\}_{k=1,2, \ldots}$ that obeyed the hypothesis of the lemma with $\kappa_{\varepsilon}$ replaced by $k^{-1}$, but not the conclusions. Given the assumptions, it follows that the subvarieties that define this sequence have uniformly bounded area where they intersect $[-3,3] \times M$. As a consequence, there is a subsequence (hence renumbered consecutively from 1) whose intersection with some neighborhood of $[-2,2] \times M$ converges pointwise to a set, $\Sigma_{0}$, with finite 2-dimensional Hausdorff measure. The subsequence can, and should be chosen so that the sequence of distributions $\left\{v \rightarrow \int_{C_{k}} v\right\}_{k=1,2, \ldots}$ on the space $C^{0}\left([-2,2] \times M ; \bigwedge^{2}([-2,2] \times M)\right)$ has a weakly convergent subsequence to a distribution. Denote the latter by $m$. The pair consisting of $\Sigma_{0}$ and $m$ defines a positive cohomology assignment. Lemma 4.4 asserts that this cohomology assignment is carried by a finite set $\vartheta$ whose typical element is a pair $(C, m)$ where $C$ is a closed, irreducible, pseudoholomorphic subvariety that is defined in some neighborhood of $[-1,1] \times M$ and where $m$ is a positive integer. Because of the manner of convergence that defines $m$, the 2 -form $d a$ must integrate to zero on the subvariety component of each pair from $\vartheta$. Since $d a$ is nonnegative on the tangent space of each such component, it follows that $d a$ is zero on each component, and so each component is $[-2,2] \times \gamma$ with $\gamma$ a Reeb orbit. The manner of convergence that defines $m$ implies that the length of each Reeb orbit involved is bounded by $\mathcal{N}$. Use $\Theta$ to denote the set of pairs $(\gamma, m)$ that arise from $\vartheta$. Given the manner of convergence of $\left\{C_{n}\right\}_{n=1,2, \ldots}$ to $\Sigma_{0}$, all points of each large $n$ version of $C_{n}$ at any point $s \in[-1,1]$ distance less than $\varepsilon$ from a fixed Reeb orbit from $\theta$, and vice versa. Furthermore, the integral of a fixed 2-form $v$ over the $s \in[-1,1]$ part of each large $n$ version of $C_{n}$ will differ by less than $\varepsilon$ from $\sum_{(\gamma, m) \in \Theta} m$. As this conclusion conflicts with the assumptions about the sequence $\left\{C_{n}\right\}_{n=1,2, \ldots}$, the lemma must be true.

This last lemma has the following corollary:

Corollary 4.7 Suppose that $\mathcal{N} \geq 1$ has been given and that each Reeb orbit of length $\mathcal{N}$ or less is nondegenerate. Given $\varepsilon>0$, there exists $\kappa_{\varepsilon}>1$ with the following significance: Let $\mathbb{I} \subset \mathbb{R}$ denote an interval of length at least 4, and suppose that $C$ is a closed, irreducible, pseudoholomorphic subvariety in a neighborhood of $\mathbb{I} \times M$ with the property that $\int_{C \cap\left(I^{\prime} \times M\right)} d a<\kappa_{\varepsilon}^{-1}$ and $\int_{C \cap\left(I^{\prime} \times M\right)} d s \wedge a \leq \mathcal{N}$ for all intervals $I^{\prime} \subset \mathbb{I}$ of length 1 . Let $I \subset \mathbb{I}$ denote the subset with distance at least 3 from any boundary 
point of $\mathbb{I}$. Then there exists a finite set $\Theta$ consisting of pairs $(\gamma, m)$ with $\gamma$ a Reeb orbit and $m$ a positive integer. This set is such that no two pair share the same Reeb orbit. In addition,

- $\sum_{(\gamma, m) \in \Theta} m \ell_{\gamma} \leq \mathcal{N}$.

- Each point of $\left.C\right|_{s}$ for $|s| \leq 1$ has distance along $M$ no greater than $\varepsilon$ from $\bigcup_{(\gamma, m) \in \Theta} \gamma$. Conversely, each point in $\bigcup_{(\gamma, m) \in \Theta} \gamma$ has distance no greater than $\varepsilon$ from $\left.C\right|_{s}$.

- If $v$ is a smooth $2-$ form on $I \times M$ with $\|v\|_{\infty}=1$ and $\|\nabla v\|_{\infty} \leq \varepsilon^{-1}$. Then

$$
\left|\int_{C \cap(I \times M)} v-\sum_{(\gamma, m) \in \Theta} m \int_{I \times \gamma} v\right| \leq \varepsilon .
$$

Proof of Corollary 4.7 This follows directly from Lemma 4.6 given the nondegeneracy assumption. Indeed, the latter implies that the Reeb orbits with length less than $2 \pi \mathcal{N}$ in $M$ are finite in number and so any two are separated by some minimal distance. Take $\varepsilon$ much less than this distance and invoke Lemma 4.6.

Part 2 The next lemma supplies a somewhat different condition that forces a pseudoholomorphic subvariety to be everywhere close to a Reeb orbit.

Lemma 4.8 Suppose that $\mathcal{N} \geq 1$ has been given and that each Reeb orbit of length $\mathcal{N}$ or less is nondegenerate. There exists $\kappa>1$, and given $\varepsilon>0$, there exists $R_{\varepsilon}>16$ with the following significance: Let $\mathbb{I} \subset \mathbb{R}$ denote an interval of length at least $2 R_{\varepsilon}$, and suppose that $C$ is a closed, irreducible, pseudoholomorphic subvariety in a neighborhood of $\mathbb{I} \times M$ with the property that $\int_{C \cap\left(I^{\prime} \times M\right)} d a<\kappa^{-1}$ and $\int_{C \cap\left(I^{\prime} \times M\right)} d s \wedge a \leq \mathcal{N}$ for all intervals $I^{\prime} \subset \mathbb{I}$ of length 1 . Let $I \subset \mathbb{I}$ denote the subset with distance at least $R_{\varepsilon}$ from any boundary point of $\mathbb{I}$. There exists a finite set $\Theta$ consisting of pairs $(\gamma, m)$ with $\gamma$ a Reeb orbit and $m$ a positive integer. This set is such that no two pair share the same Reeb orbit. In addition,

- $\sum_{(\gamma, m) \in \Theta} m \ell_{\gamma} \leq \mathcal{N}$.

- Each point of $\left.C\right|_{s}$ for $s \in I$ has distance along $M$ no greater than $\varepsilon$ from $\bigcup_{(\gamma, m) \in \Theta} \gamma$. Conversely, each point in $\bigcup_{(\gamma, m) \in \Theta} \gamma$ has no greater than $\varepsilon$ from $\left.C\right|_{s}$.

- If $I^{\prime} \subset I$ is an interval of length 1 and $v$ is a smooth 2-form on $I^{\prime}$ with $\|v\|_{\infty}=1$ and $\|\nabla v\|_{\infty} \leq \varepsilon^{-1}$, then

$$
\left|\int_{C \cap\left(I^{\prime} \times M\right)} v-\sum_{(\gamma, m) \in \Theta} m \int_{I^{\prime} \times \gamma} v\right| \leq \varepsilon .
$$


Proof of Lemma 4.8 Fix $\delta>0$, but very much less than the distance between any two distinct Reeb orbits with length less than $\mathcal{N}$. Let $I^{*} \subset \mathbb{I}$ denote the set of points with distance at least 4 from each boundary point. Let $\kappa=\kappa_{\delta}$ denote the constant provided by Corollary 4.7. Suppose that $C$ denotes a subvariety that obeys the assumptions of Corollary 4.7 with $\delta$ used in lieu of $\varepsilon$, and let $\Theta_{C}$ denote the set of pairs of Reeb orbit and positive integer that Corollary 4.7 provides. Note that the collection of such sets is finite since the Reeb orbits with length at most $\mathcal{N}$ are nondegenerate. Let $\Lambda_{\mathcal{N}}$ denote this set.

Now suppose that $\varepsilon<\delta$ and that there is no $R_{\varepsilon}$ as claimed by the lemma. Given that $\Lambda_{\mathcal{N}}$ is finite, there exists $\Theta \in \Lambda_{\mathcal{N}}$, a sequence $\left\{\mathbb{I}_{k}\right\}_{k=1,2, \ldots}$ of intervals of length $32 k$, and a sequence $\left\{C_{k}\right\}_{k=1,2, \ldots}$ where any given $C_{k}$ is a closed, irreducible, pseudoholomorphic subvariety from a neighborhood of $\mathbb{I}_{k} \times M$ with the property that $\int_{C \cap\left(I^{\prime} \times M\right)} d a \leq \kappa_{\delta}^{-1}$ and with $\int_{C \cap\left(I^{\prime} \times M\right)} d s \wedge a \leq \mathcal{N}$ for each interval $I^{\prime} \subset \mathbb{I}_{k}$ of length 1 . Moreover, one or both of the following is true:

- There is a point in each $C_{k} \cap\left(\mathbb{I}_{k} \times M\right)$ with distance $k$ or more from a boundary point of $I_{k} \times M$ and with distance $\varepsilon$ or greater from $\bigcup_{(\gamma, m) \in \Theta} \mathbb{I}_{k} \times \gamma$. Or, there is a point in this union with distance $k$ or more from a boundary point, and with distance $\varepsilon$ or more from $C$.

- There is an interval $I_{k}^{\prime} \subset \mathbb{I}$ with length 1 and distance $k$ or more from a boundary point of $I_{k}$ and a smooth 2 -form $v_{k}$ on $I_{k}^{\prime}$ with $\left\|v_{k}\right\|_{\infty}=1$ and $\left\|\nabla v_{k}\right\|_{\infty} \leq \varepsilon^{-1}$ that violates the inequality asserted in the lemma.

Suppose first that the top item in (4-11) is obeyed. By translating $I_{k}$ and $C_{k}$ along the $\mathbb{R}$ factor of $\mathbb{R} \times M$, one can arrange that such a point sits where $s=0$. Assume that this is the case. Taking limits, as in the proof of Lemma 4.6, finds a subsequence (to be renumbered consecutively from 1) that converges pointwise on bounded domains inside $\mathbb{R} \times M$ to a pseudoholomorphic subvariety, $C_{*} \subset \mathbb{R} \times M$ with the following properties: Each point of $C_{*}$ has distance $\delta$ or less from $\bigcup_{(\gamma, m) \in \Theta} \mathbb{R} \times \gamma$. But, there exists a point on $C_{*}$ where $s=0$ with distance greater than $\varepsilon$ from $\bigcup_{(\gamma, m) \in \Theta} \mathbb{R} \times \gamma$ or vice-versa. Given that each Reeb orbit from $\Theta$ is isolated, it follows that $\lim _{|s| \rightarrow \infty}\left(\sup _{\left.z \in C_{*}\right|_{s}} \operatorname{dist}\left(z, \bigcup_{(\gamma, m) \in \Theta} \gamma\right)\right)=0$. But this is not possible since integration by parts finds the integral of $d a$ over $C_{*}$ to be zero under these assumptions.

Suppose next that the bottom item in (4-11) is obeyed but not the top. Translate each $I_{k}$ and $C_{k}$ along the $\mathbb{R}$ factor of $\mathbb{R} \times M$ so that $I_{k}^{\prime}=[0,1]$. Use $C_{k}$ to denote now the translated version of the original. By virtue of the Arzela-Ascoli theorem, there is a subsequence of $\left\{C_{k}\right\}_{k=1,2, \ldots}$ (hence renumbered consecutively from 1) with the following property: Let $\left\{v_{k}\right\}_{k=1,2, \ldots}$ now denote the corresponding sequence 
of translated 2-forms. This sequence has a subsequence that converges strongly in $C^{0}\left([0,1] \times M ; \bigwedge^{2}([0,1] \times M)\right.$ to a form $v_{\infty}$ with sup-norm 1 . The latter is Lipschitz with Lipschitz constant $\varepsilon^{-1}$. Meanwhile, the arguments given for Lemma 4.6 can be used to prove that the sequence of distributions $\left\{v \rightarrow \int_{C_{k} \cap([0,1] \times M)} v\right\}$ has a subsequence that converges weakly to the distribution given by $v \rightarrow \sum_{(\gamma, m)} m \int_{[0,1] \times \gamma} v$ where $m$ is a positive integer. But this sort of convergence is not possible given the properties of $v_{\infty}$.

Thus, neither of the possibilities in (4-11) are allowed, so the lemma must be true.

Part 3 Return to the context of Proposition 4.5 and its set $\mathbb{I}$. The first point to make is that if $r \geq c_{0}$ and $\left[s^{\prime}, s^{\prime}+1\right] \in \mathbb{I}$, then

$$
\left|\frac{i}{2 \pi} \int_{\left[s^{\prime}, s^{\prime}+1\right] \times M} d s \wedge a \wedge F_{\hat{A}}\right| \leq c_{0} \mathcal{K} .
$$

This follows from Lemmas 3.1, 3.8 and Lemma 3.9. Meanwhile, what with (3-34) and (3-35), it follows from an integration by parts that

$$
\frac{i}{2 \pi} \int_{I^{\prime} \times M} d a \wedge F \hat{A} \leq c_{0} \mathcal{K}
$$

if $I^{\prime} \subset \mathbb{I}$ is any given closed subset and $r \geq c_{0}$. Keep these inequalities in mind in what follows.

Lemma 4.9 Given $\mathcal{K} \geq 1$, suppose that each Reeb orbit with length $(1 /(2 \pi)) \mathcal{K}$ or less is nondegenerate. Given also $\varepsilon>0$, there exists $\kappa \geq 1$ with the following significance: Suppose that $r \geq \kappa$ and that $(A, \psi=(\alpha, \beta))$ is an instanton solution to (1-11) with either $\mathrm{A}_{\mathfrak{d}}<r^{2}$ or $\mathrm{F}_{\mathfrak{d}} \geq-r^{2}$. Let $\mathbb{I} \subset \mathbb{R}$ denote a connected subset of length at least 16 with $\underline{\mathrm{M}}(s) \leq \mathcal{K}$ on $\mathbb{I}$. Let $\mathcal{I}$ denote the set of $k \subset Z$ with $[k, k+1] \in \mathbb{I}$ and with $(i /(2 \pi)) \int_{[k, k+1] \times M} d a \wedge F_{\hat{A}} \geq \varepsilon$. Let $I^{\prime} \subset \mathbb{I}-\left(\bigcup_{k \in I}[k, k+1]\right)$ denote any given component. Then $-\varepsilon^{2} \leq(i /(2 \pi)) \int_{I^{\prime} \times M} d a \wedge F \hat{A}$.

Proof of Lemma 4.9 Suppose that $\delta>0$ has been given, and that $s \in I^{\prime}$ is a point with distance at least $\delta^{-1}+8$ from any boundary point of $I^{\prime}$. Invoke Proposition 4.1 using in lieu of $\mathbb{I}$ the interval $\mathbb{I}_{s}=\left[s-\delta^{-1}-8, s+\delta^{-1}+8\right]$. Given that $r$ is greater than some $\delta$-dependent constant, this proposition supplies an $\mathbb{I}_{s}$ version of its set $\vartheta$, this denoted by $\vartheta_{s}$. Thus, the typical element in $\vartheta_{s}$ is a pair $(C, m)$ with $m$ a positive integer and $C$ a closed, pseudoholomorphic subvariety that is defined in a neighborhood of $\left[s-\delta^{-1}, s+\delta^{-1}\right]$. Let $I^{\prime \prime} \subset\left[s-\delta^{-1}, s+\delta^{-1}\right]$ denote a length 1 subcylinder. Then the integral of $d a$ over $\mathbb{C}^{\prime}$ 's intersection with $I^{\prime \prime} \times M$ is less than $\varepsilon+\delta$. Let $\kappa \geq 1$ be as described in Lemma 4.8 and assume now that $\varepsilon+\delta$ is less than $\kappa^{-1}$. Fix $\varepsilon^{\prime}>0$ 
and let $R_{\mathcal{E}^{\prime}}$, denote the constant from Lemma 4.8. Suppose that $\delta^{-1}>2 R_{\mathcal{E}^{\prime}}$. Lemma 4.8 asserts that the each point of $C$ in the set $\left[s-\delta^{-1}+R_{\mathcal{\varepsilon}^{\prime}}, s+\delta^{-1}-R_{\mathcal{E}^{\prime}}\right] \times M$ has distance $\varepsilon^{\prime}$ or less from $\mathbb{R} \times \gamma$ where $\gamma$ is a Reeb orbit with length no greater than $\mathcal{K}$. As a consequence, each point of $\alpha^{-1}(0)$ with distance at least $R_{\mathcal{E}^{\prime}}+16$ from any boundary point of $I^{\prime}$ has distance $\varepsilon^{\prime}$ or less from some $\mathbb{R} \times \gamma$.

To continue fix $s \in I^{\prime}$ with distance at least $R_{\mathcal{\varepsilon}^{\prime}}+16$ from any boundary point of $I^{\prime}$. The integral of $(i /(2 \pi)) a \wedge F_{\hat{A}}$ over the portion of $\{s\} \times M$ with distance $2 \varepsilon^{\prime}$ or less from $\gamma$ can be written using the coordinates that are used in (1-15) as

$$
\frac{\ell_{\gamma}}{4 \pi^{2}} i \int_{t \in S^{1}}\left(\left.\int_{|z|<2 \varepsilon^{\prime}} F_{\hat{A}}\right|_{t}\right) d t+\mathfrak{e},
$$

where $|\mathfrak{e}| \leq c_{0} \mathcal{K} \varepsilon^{\prime}$. This bound on $\mathfrak{e}$ follows using the Lemmas 3.1, 3.6, 3.8 with Lemma 3.9. Meanwhile, it follows from Proposition 4.1 and (3-35) that the integral term in (4-14) is equal to $m \ell_{\gamma}+\mathfrak{e}^{\prime}$ where $\left|\mathfrak{e}^{\prime}\right| \leq c_{0} r^{-1 / 2}$.

Granted what just said, it follows using an integration by parts that the integral of $(i /(2 \pi)) d a \wedge F_{\hat{A}}$ over the portion of $I^{\prime} \times M$ with distance at least $R_{\mathcal{E}^{\prime}}+16$ from any boundary point of $I^{\prime}$ is no less than $-c_{0}\left(\mathcal{K} \varepsilon^{\prime}+r^{-1 / 2}\right)$.

A lower bound for the integral over the rest of $I^{\prime} \times M$ is obtained as follows: Note first that Lemmas 3.1, 3.6, 3.8 and 3.9 imply that

$$
-c_{0} r^{-1} \leq \frac{i}{2 \pi} \int_{\{s\} \times M} d a \wedge F_{\hat{A}}
$$

at each point $s \in \mathbb{R}$. Note as well, that the bottom equation in (1-11) implies that

$$
\frac{i}{2 \pi} d a \wedge \nabla_{A} \alpha \wedge \nabla_{A} \bar{\alpha} \geq-c_{0}\left|\nabla_{A} \beta\right|^{2} .
$$

Given the bound in (4-15), it follows that the integral of $(i /(2 \pi)) d a \wedge F_{\hat{A}}$ over the remaining portion of $I^{\prime} \times M$ is no less than $c_{0} R_{\mathcal{E}^{\prime}} r^{-1}$.

Thus, the integral over $I^{\prime} \times M$ is no less than $-c_{0}\left(\mathcal{K}_{\varepsilon^{\prime}}+R_{\mathcal{E}^{\prime}} r^{-1}+r^{-1 / 2}\right)$ if $r$ is greater than some $\varepsilon^{\prime}$ dependent constant. This understood, choose $\varepsilon^{\prime}<\frac{1}{2} c_{0}^{-1} \mathcal{K}^{-1} \varepsilon^{2}$. Granted what was said above, the desired bound for the integral of $(i /(2 \pi)) d a \wedge F_{\hat{A}}$ follows if $r$ is greater than some purely $\varepsilon$-dependent constant.

Part 4 With $\varepsilon>0$ fixed and much less than 1, take $r$ large enough so as to invoke Lemma 4.9. Let $\mathcal{I}=\mathcal{I}_{\varepsilon}$ be as described in Lemma 4.9. Fix $R \gg 1$ and consider the integral on the left hand side of (4-13) for $I^{\prime}=[-R, R] \times M$. Let $N_{R}$ denote the number of integers $k \in \mathcal{I}$ with $[k, k+1] \subset[-R, R]$. The contribution to the $[-R, R]$ version of the left hand side of (4-13) from the cylinders $[k, k+1] \subset[-R, R]$ with 
$k \in \mathcal{I}_{\varepsilon}$ sums to at least $N_{R} \varepsilon$. Meanwhile, Lemma 4.9 implies that the contribution from the rest of $[-R, R] \times M$ is no less than $-\left(N_{R}+1\right) \varepsilon^{2}$. This understood, it follows from (4-13) that $N_{R} \leq c_{0} \mathcal{K} \varepsilon^{-1}$. As this last bound is independent of $R$, there are at most this many integers in $\mathcal{I}_{\varepsilon}$. Let $N_{\varepsilon}$ denote the number of elements in $\mathcal{I}_{\varepsilon}$.

Fix $L>\delta^{-1}$ and for each $s \in \mathbb{I}$ with distance at least $L+7$ from any boundary point of $\mathbb{I}$, let $\mathbb{I}_{s}=[s-L, s+L]$. Given $L$, Proposition 4.1 can be invoked if $r \geq \kappa_{L} \geq 1$ where $\kappa_{L}$ depends only on $\mathcal{K}, f$ and $L$. In particular, Proposition 4.1 supplies its set $\vartheta=\vartheta_{s}$. Suppose that $s$ has distance at least $L+1$ from $\mathcal{I}_{\varepsilon}$. If $\varepsilon \leq c_{0}^{-1}$ and if $L=4 R_{\delta}$ where $R_{\delta}$ is defined from Lemma 4.9, then Lemma 4.9 can be used to replace any $s \in \mathbb{I}_{s}$ version of $\vartheta_{s}$ with a collection $\vartheta_{s}^{\prime}$ whose typical element is a pair $(\mathbb{R} \times \gamma, m)$ with $\gamma$ a Reeb orbit of length less than $\mathcal{K}$ and with $m$ a positive integer such $\sum_{(m, \gamma)} m \ell_{\gamma}$. This replacement of $\vartheta_{s}$ with $\vartheta_{s}^{\prime}$ has the following cost: The conclusions of Proposition 4.1 hold using $\vartheta_{s}$ only on $\left[s-3 R_{\delta}, s+3 R_{\delta}\right]$.

Let $\mathbb{I}^{\prime} \subset \mathbb{I}$ denote the set of points with distance less than $6 R_{\delta}$ from $\mathcal{I}_{\varepsilon}$. This set is a disjoint union of at most $N_{\varepsilon}$ intervals whose total length is at most $6 R_{\delta} N_{\varepsilon}$. Given that $\varepsilon$ is fixed already in a $\delta$-independent fashion, Proposition 4.1 can be applied to each such interval if $r \geq \kappa_{\delta}^{\prime}>1$ to supply for each a set $\vartheta$. This understood, the conclusions of Proposition 4.5 are satisfied using some $N^{\prime} \leq N_{\varepsilon}$ versions of $\vartheta$ to deal with the components of $\mathbb{I}^{\prime}$, while using for $\mathbb{I}-\mathbb{I}^{\prime}$ at most $N^{\prime}+1$ versions of sets $\vartheta$ whose constituent pairs have subvariety component $\mathbb{R} \times \gamma$.

Part 5 The assumption is that $\mathbb{I}$ has the form $\left[s_{0}, \infty\right)$ or $(-\infty, \infty)$. To prove the assertion made by the third bullet about $\mathbb{I}$, fix $\varepsilon>0$ and very small. Let $\mathcal{I}$ denote the subset of $k \in \mathbb{Z} \cap \mathbb{I}$ with $(i /(2 \pi)) \int_{[k, k+1] \times M} d a \wedge F_{\hat{A}}>\varepsilon$. Note that $\mathcal{I}$ is bounded. Let $\Lambda$ denote the set of components of $\mathbb{I}-\left(\bigcup_{k \in \mathcal{I}}[k, k+1]\right)$ with length at least 16 . This is a finite set because the $s \rightarrow \pm \infty$ limits of $\mathfrak{d}$ are solutions to (1-8). Let $N$ denote the number of elements in $\Lambda$. Order the set $\Lambda$ as $\left\{I_{0}^{\prime}, \ldots, I_{N}^{\prime}\right\}$ with the ordering given by the value of the supremum of $s$. Thus, $I_{N}^{\prime}$ is unbounded from above. With the preceding understood, the remainder of the proof is broken into four steps.

Step 1 Let $s_{N-}$ denote the minimum point in $I_{N}^{\prime}$ or $-\infty$ if $I_{N}^{\prime}=\mathbb{R}$. Fix $\delta_{0}>0$ but small. Apply Proposition 4.1 to the intervals in $I$ of length $2 \delta_{0}^{-1}+16$ with a point from $I_{N}^{\prime}$. Then apply Lemma 4.6 to all subintervals in $I_{N}^{\prime}$ of length 1 . Given the conclusions of Proposition 4.1, there exists $r_{0}$ that depends only on $\delta_{0}$ and is such that if $r>r_{0}$, then Lemma 4.6 assigns to any such subinterval a collection of pairs of Reeb orbit and positive integer weight. Given that the Reeb orbits of length $E_{+}$or less are nondegenerate, the conclusions of Lemma 4.6 applied to overlapping length 1 intervals starting at any given, sufficiently large value of $s$ and then working towards 
smaller values leads to the following conclusion when $\delta_{0} \leq c_{0}^{-1}$ and $\varepsilon<c_{0}^{-1}$, and $r$ is large: There exists a finite set $\Theta$ consisting of pairs $(\gamma, m)$ with $\gamma$ a Reeb orbit and $m$ a positive integer. This set is such that no two distinct pairs share the same Reeb orbit. Furthermore, $\sum_{(\gamma, m) \in \Theta} m \ell_{\gamma} \leq(1 /(2 \pi)) \mathrm{E}_{+}$and the assertions of the two bullets of Proposition 4.1 hold using the given value of $\delta_{0}$ for the whole of $I=I_{N}^{\prime}$ for large $r$ with the set $\vartheta=\{(\mathbb{R} \times \gamma, m)\}_{(\gamma, m) \in \Theta}$.

Step 2 If $N=1$ and so $I_{N}^{\prime}=I$, then nothing more need be said. Assume that this is not the case. Apply Proposition 4.1 to overlapping subintervals of length $2 \delta_{0}^{-1}+16$ in $I$ with a point from $I_{N-1}^{\prime}$. Then apply Lemma 4.6 to overlapping subintervals of length 1. These assign to each such subinterval a finite collection of pairs of Reeb orbit and positive integer if $\delta_{0}, \varepsilon<c_{0}^{-1}$ and $r>r_{0}$. To elaborate, let $\hat{I}$ denote a given such length 1 interval and let $\Theta$ denote the assigned collection. The conclusions of Proposition 4.1 hold for the $I=\hat{I}$, with constant $\delta$ equal to $\delta_{0}$ when $r \geq r_{0}$, and with $\vartheta=\{(\mathbb{R} \times \gamma, m)\}_{(\gamma, m) \in \Theta}$.

Let $S_{(N-1)+}$ denote the maximal point of $I_{N-1}^{\prime}$ and let $S_{N-}$ denote the minimal point of $I_{N}^{\prime}$. Let $\Theta^{\prime}$ denote the collection of Reeb orbits assigned to $\hat{I}_{-}=$ $\left[s_{(N-1)+}-1, s_{(N-1)+}\right]$. Let $S_{N-}$ denote the minimal point in $I_{N}^{\prime}$ and introduce $\widehat{I}_{+}$to denote $\left[S_{N_{-}}, S_{N_{-}}+1\right]$. Let $\sigma$ denote the piecewise differentiable function on $R$ that is zero for $s<s_{(N-1)+}-1$, equals $s-s_{(N-1)+}$ on $\hat{I}_{-}$is equal to 1 on $\left[s_{(N-1)+}, s_{N-}\right]$, equals $s_{N-}+1-s$ on $\hat{I}_{+}$and is zero for $s>s_{N-}+1$. Integrate the 4-form $(i /(2 \pi)) d \sigma \wedge a \wedge F_{A}$ on $\mathbb{R} \times M$ and integrate by parts using (4-15) to see that

$$
\frac{i}{2 \pi} \int_{\widehat{I}_{+} \times M} d s \wedge a \wedge F_{\hat{A}}-\frac{i}{2 \pi} \int_{\widehat{I}_{-} \times M} d s \wedge a \wedge F_{\hat{A}} \geq-c_{0}\left(\varepsilon+\delta_{0}\right)
$$

when $r \geq c_{0}$. Granted this, granted the conclusions of Step 1, and granted what is said by Proposition 4.1, it then follows that $\sum_{(\gamma, m) \in \Theta^{\prime}} m \ell_{\gamma} \leq(1 /(2 \pi)) \mathrm{E}_{+}$if $\delta_{0}$ and $\varepsilon$ are less than $c_{0}^{-1}$.

With the preceding understood, it then follows using the argument from Step 1 for $I_{N-1}^{\prime}$ that each subinterval of length 1 in $I_{N-1}^{\prime}$ is assigned this same collection $\Theta^{\prime}$ by Lemma 4.6. As a consequence, the two bullets of Proposition 4.1 hold for $r \geq r_{0}$ with $\delta=\delta_{0}$, with $I=I_{N-1}^{\prime}$, and with the set $\vartheta=\{(\mathbb{R} \times \gamma, m)\}_{(\gamma, m) \in \Theta^{\prime}}$.

Step 3 Repeat Step 2 first with the roles of the pair $\left(I_{N}^{\prime}, I_{N-1}^{\prime}\right)$ played by the pair $\left(I_{N-1}^{\prime}, I_{N-2}^{\prime}\right)$, and then by $\left(I_{N-2}^{\prime}, I_{N-3}^{\prime}\right)$, and so on to draw the following conclusion: For any $k \in\{1, \ldots, N\}$, there is a finite set $\Theta_{k}$ of pairs $(\gamma, m)$ of Reeb orbit and positive integer such that no two pair share the same Reeb orbit, 
such that $\sum_{(\gamma, m) \in \Theta_{k}} m \ell_{\gamma} \leq(1 /(2 \pi)) \mathrm{E}_{+}$, and such that the two bullets of Proposition 4.1 hold for $r \geq r_{0}$, with $\delta=\delta_{0}$, with $I$ being the whole of $I_{k}^{\prime}$ with the set $\vartheta=\vartheta_{k}^{\prime}=\{(\mathbb{R} \times \gamma, m)\}_{(\gamma, m) \in \Theta_{k}}$.

Granted the preceding, it follows that Lemma 4.9 can be invoked when $r \geq r_{0}$ with each $I_{k}^{\prime}$ used instead of $\mathbb{I}$ and with $\mathrm{E}_{+}$used instead of $\mathcal{K}$. Doing so leads to the conclusion that the integer $N$ is bounded apriori given $\mathcal{K}$.

Step 4 Given the conclusions of this last step, then the arguments in Part 4 can be used with only cosmetic changes to prove that the assertions of the first two bullets of Proposition 4.5 hold with the assumption that all Reeb orbits of length less than $(1 /(2 \pi)) \mathrm{E}_{+}$ are nondegenerate. Meanwhile, the assertion that $\lim _{s \rightarrow \infty} \mathrm{E}\left(\left.\mathfrak{d}\right|_{s}\right) \leq \mathrm{E}_{+}+\delta$ follows from the $I_{1}^{\prime}$ version of Step 3 using Item (2)(b) of the second bullet of Proposition 4.5.

\section{4.e Local structure}

This subsection says something about how the points in $\alpha^{-1}(0)$ vary when this locus is very near a pseudoholomorphic subvariety. The results are summarized in Lemma 4.10 below. The latter is the analog here of Lemma 5.4 in the article $\mathrm{SW}=\mathrm{Gr}$ from [6].

The lemma refers to a connected component, $C$, of the $|s| \leq 2$ part of a pseudoholomorphic submanifold that is defined on some neighborhood of $[-2,2] \times M$ in $\mathbb{R} \times M$. Assume in what follows that $\{ \pm 1\}$ are regular values of $s$ on $C$. To further set the stage for the lemma, let $\pi: N \rightarrow C$ denote $C$ 's normal bundle. There exists $\rho_{1}>0$ and an exponential map as described in $\left[9\right.$, Section 4.a] on the radius $\rho_{1}$ disk bundle in $N$ that embeds this disk bundle. Introduce $N_{1}$ to denote the radius $\rho_{1}$ disk bundle and use $e_{C}: N_{1} \rightarrow \mathbb{R} \times M$ to denote the exponential map. Note that $e_{C}$ embeds each fiber disk of $N_{1}$ as a pseudoholomorphic disk and that its differential is an isometry along $C$. Use $N_{1}^{\prime}$ and $N_{1}^{\prime \prime}$ to denote the respective radius $\frac{1}{2} \rho_{1}$ and radius $\frac{1}{4} \rho_{1}$ subbundle in $N_{1}$.

To continue with the stage setting, suppose that $p \subset C$ is any given point. If $D \subset \mathbb{C}$ is an embedded disk centered at $p$, use parallel transport by the Hermitian connection to identify $\left.N\right|_{D}$ as $D \times\left. N\right|_{p}$.

Here is one final piece of notation: If $(A, \psi)$ is an instanton, use $S$ in what follows to denote the $|s| \leq 1$ part of the intersection between $\alpha^{-1}(0)$ and $e_{C}\left(N_{1}\right)$.

Lemma 4.10 Fix $C$ is as described above. Given $\varepsilon>0$ and $\mathcal{K} \geq 1$, there exists $\kappa \geq 1$ such that the following is true: Suppose that $r \geq \kappa$, and that $\mathfrak{d}=(A, \psi=$ $(\alpha, \beta))$ is an instanton solution to (1-11) with either $\mathrm{A}_{\mathfrak{d}}<r^{2}$ or $\mathrm{F}_{\mathfrak{d}} \geq-r^{2}$, and with $\sup _{s \in[-8,8]} \underline{\mathrm{M}}(s) \leq \mathcal{K}$. Assume that the intersection of $\alpha^{-1}(0)$ with $e_{C}\left(N_{1}\right)$ lies 
$e_{C}\left(N_{1}^{\prime}\right)$. Let $m \geq 1$ denote the integral of $(i /(2 \pi)) F_{\hat{A}}$ on any fiber disk of $N_{1}^{\prime}$. Let $\delta$ denote the greater of the numbers $r^{-1 / 2}$ and $\sup _{z \in C \cap([-1,1] \times M)} \operatorname{dist}(z, S)$. Assume that $\delta<\kappa^{-1}$. Let $p \in C$ denote a point where $|s| \leq 1$ and let $D \subset \mathbb{C}$ denote the disk of radius $\delta$ centered at $p$. There exists a set of $m$ or fewer points $\left.\Lambda \subset N_{1}\right|_{p}$ such that if $z \in D$, then each point in $S \cap e_{C}\left(\left.N_{1}\right|_{z}\right)$ has distance at least $\varepsilon \delta$ from $e_{C}\left(\left.\Lambda\right|_{z}\right)$.

Proof of Lemma 4.10 The proof is essentially identical but for one small aspect to the proof of Lemma 5.4 in the article $\mathrm{SW}=\mathrm{Gr}$ from [6]. To explain the difference, start as in the proof of Lemma 5.4 in this same $\mathrm{SW}=\mathrm{Gr}$ article by assuming the lemma is false so as to derive nonsense. There then exist sequences $\left\{\left(r_{n},\left(A_{n}, \psi_{n}\right)\right)\right\}_{n=1,2, \ldots},\left\{\delta_{n}\right\}_{n=1,2, \ldots}$ and $\left\{p_{n}\right\} \subset C \cap([-1,1] \times M)$ with the following properties: First, the sequence $\left\{r_{n}\right\}_{n=1,2, \ldots} \subset[1, \infty)$ is increasing and unbounded and the sequence $\left\{\delta_{n}\right\}_{n=1,2, \ldots} \subset\left(0, \frac{1}{4} \rho_{0}\right)$ is decreasing with limit zero. Second, any given $\left(r_{n},\left(A_{n}, \psi_{n}=\left(\alpha_{n}, \beta_{n}\right)\right)\right.$ obeys the assumptions of the lemma with $\delta=\delta_{n}$, but is such that there is no set of $m$ or fewer points in $\left.N_{1}\right|_{p_{n}}$ that makes the conclusions of the lemma hold. Third, the sequence $\left\{p_{n}\right\}_{n=1,2, \ldots}$ converges.

To proceed from here, fix complex Gaussian coordinates centered at $p_{n}$ of the sort that is described in Part 2 of Section 4.a, but chosen so that the coordinates, $x=\left(x_{0}, x_{1}\right)$, are such that the plane $x_{1}=0$ is tangent to $C$ at $z_{n}$ and the plane $x_{0}=0$ is tangent to $e_{C}\left(N_{1}\right)$ at $p_{n}$. Let $\rho_{*}>0$ be such that the coordinates are defined for $|x| \leq \rho_{*}$. There are two cases to consider. In the first case is that where $\left\{\delta_{n} r_{n}^{1 / 2}\right\}_{n=1,2, \ldots}$ has a bounded and thus convergent subsequence. In this case, pull $\left(A_{n}, \psi_{n}\right)$ back to the radius $r_{n}^{1 / 2} \rho_{*}$ ball in $\mathbb{C}^{2}$ by the map $x \rightarrow r_{n}^{1 / 2} x$. If there is no bounded subsequence of $\left\{\delta_{n} r_{n}^{1 / 2}\right\}_{n=1,2, \ldots}$, pull $\left(A_{n}, \psi_{n}\right)$ back to the radius $\delta_{n}^{-1} \rho_{*}$ ball in $\mathbb{C}^{2}$ by the map $x \rightarrow \delta_{n} x$. In either case, view the result as a pair $\left(A_{n \delta}, \psi_{n \delta}\right)$ of connection on the trivial $\mathbb{C}$ bundle and spinor on a ball of radius $\rho_{n}$ in $\mathbb{C}^{2}$. In either case, the sequence $\left\{\rho_{n}\right\}_{n=1,2, \ldots}$ is unbounded.

As argued in the proof of Lemma 5.4 in the article $\mathrm{SW}=\mathrm{Gr}$ from [6], there is a complex algebraic subvariety $\Sigma \subset \mathbb{C}^{2}$ and a subsequence of $\left\{\left(A_{n}, \psi_{n}\right)\right\}_{n=1,2, \ldots}$ (hence renumbered consecutively from 1) with the following properties: First, $\Sigma$ has intersection number no greater than $m$ with any complex line that is not contained in $\Sigma$. Second, the sequence

$$
\lim _{R \rightarrow \infty} \lim _{n \rightarrow \infty}\left(\sup _{\substack{x \in \Sigma \\|x| \leq R}} \operatorname{dist}\left(x, \alpha_{n \delta}^{-1}(0)\right)+\sup _{\substack{z \in \alpha_{n \delta}^{-1}(0) \\|x| \leq R}}(\Sigma, x)\right)
$$

converges with limit zero. Third, every point in $\Sigma$ has distance 1 or less from the $x_{1}=0$ plane unless the sequence $\left\{p_{n}\right\}_{n=1,2, \ldots}$ converges to a point where $|s|=1$. In 
the latter case, there exists a unit length complex number $\sigma$, and every point in $\Sigma$ has distance 1 or less from the $x_{1}=0$ plane where real $\left(\sigma x_{0}\right) \geq 0$. (The latter case does not arise in the case studied in $\mathrm{SW}=\mathrm{Gr}$.)

The only algebraic subvarieties that obey the third property are disjoint unions of at most $m$ parallel planes with $x_{1}=$ constant. However, if $\Sigma$ is of the latter sort, then the manner of convergence implies that the conclusions of the lemma are not violated when $n$ is large.

\section{Instantons and pseudoholomorphic subvarieties: Part II}

Fix again a pair $(a, J)$ and then $\mu$ so as to define (1-11)). Here as before, $\mu$ has $\mathcal{P}$-norm bounded by 1 . The upcoming Proposition 5.1 states the first results from this section. The main result in this subsection is Proposition 5.5 in Section 5.e, this a stronger version of Proposition 5.1.

Proposition 5.1 says the same thing as the $\mathbb{I}=\mathbb{R}$ version of Proposition 4.5 but for one very important difference: Proposition 4.5 's requirement that $\sup _{s \in \mathbb{R}} \underline{\mathrm{M}}(S) \leq \mathcal{K}$ is replaced by a condition on the $s \rightarrow \infty$ limit of the instanton: $\mathrm{E}_{+}=\lim _{s \rightarrow \infty} \mathrm{E}\left(\left.A\right|_{s}\right) \leq \mathcal{K}$. Here, $\mathrm{E}$ is the function on $\operatorname{Conn}(E)$ that is depicted in (1-9). Recall that when $\mathfrak{d}$ is a given instanton, then $f_{\mathfrak{d}}$ denotes the spectral flow from its $s \rightarrow-\infty$ limit to its $s \rightarrow \infty$ limit.

Proposition 5.1 Fix $(A, J)$ as above. Given $\mathcal{K} \geq 1$ and $\mathrm{E}_{+} \leq \mathcal{K}$, there exists $\kappa \geq 1$, and given $\delta>0$, there exists $\kappa_{\delta} \geq 1$ which, with $\kappa$, has the following significance: Suppose that $r \geq \kappa_{\delta}$, and that $\mathfrak{d}=(A, \psi=(\alpha, \beta))$ is an instanton solution to (1-11). If $c_{1}(\operatorname{det}(\mathbb{S}))$ is a torsion class, assume $\mathrm{A}_{\mathfrak{d}} \leq \mathcal{K} r$ or $f_{\mathfrak{d}} \geq-\kappa^{-1} r^{2}$. If $c_{1}(\operatorname{det}(\mathbb{S}))$ is not a torsion class, assume that $\mathrm{A}_{\mathfrak{d}} \leq \mathcal{K} r$ or that $f_{\mathfrak{d}} \geq-\mathcal{K} r$. In either case, assume that $\lim _{s \rightarrow \infty} \mathrm{E}\left(\left.A\right|_{s}\right) \leq \mathrm{E}_{+}$and require that all Reeb orbits with length at most $(1 /(2 \pi)) \mathrm{E}_{+}$ are nondegenerate.

- Let $\mathfrak{c}_{-}=\left.\lim _{s \rightarrow-\infty}(A, \psi)\right|_{s}$. Then $\mathfrak{c}_{-}$is a solution to $(1-11)$ with $\mathrm{E}\left(\mathfrak{c}_{-}\right) \leq$ $\mathrm{E}_{+}+\delta$.

- Each point in $\mathbb{R} \times M$ where $|\alpha| \leq 1-\delta$ has distance at most $\kappa r^{-1 / 2}$ from where $\alpha=0$.

- Moreover, there exists

(a) A positive integer $N \leq \kappa$ and a cover of $\mathbb{R}$ as $\bigcup_{1 \leq k \leq N} I_{k}$ by connected open sets of length at least $2 \delta^{-1}$. These are such that $I_{k} \cap I_{k^{\prime}}=\varnothing$ if $\left|k-k^{\prime}\right|>1$. In addition, if $\left|k-k^{\prime}\right|=1$, then $I_{k} \cap I_{k^{\prime}}$ has length between $\frac{1}{128} \delta^{-1}$ and $\frac{1}{64} \delta^{-1}$. 
(b) For each $k \in\{1,2, \ldots, N\}$, a set $\vartheta_{k}$ whose typical element is a pair $(C, m)$ where $m$ is a positive integer and where $C \subset \mathbb{R} \times M$ is a pseudoholomorphic subvariety defined in a neighborhood of $I_{k} \times M$. These elements of $\vartheta_{k}$ are constrained so that

$$
\sum_{(C, m) \in \vartheta_{k}} m \int_{\mathbb{C}} d a<\kappa
$$

In addition, these sets $\left\{\vartheta_{k}\right\}_{k=1, \ldots, N}$ are such that

(1) $\sup _{\substack{z \in \bigcup_{(C, m) \in \vartheta_{k}} C, s(z) \in I_{k}}} \operatorname{dist}\left(z, \alpha^{-1}(0)\right)+\sup _{\substack{z \in \alpha^{-1}(0), s(z) \in I_{k}}} \operatorname{dist}\left(\bigcup_{(C, m) \in \vartheta_{k}} C, z\right)<\delta$.

(2) Let $k \in\{1, \ldots, N\}$, let $I^{\prime} \subset I_{k}$ denote an interval of length 1 , and let $v$ denote the restriction to $I^{\prime} \times M$ of a 2 -form on $\mathbb{R} \times M$ with $\|v\|_{\infty}=1$ and $\|\nabla v\|_{\infty} \leq \delta^{-1}$. Then

$$
\left|\frac{i}{2 \pi} \int_{I^{\prime} \times M} v \wedge F_{\hat{A}}-\sum_{(C, m) \in \vartheta} m \int_{C} v\right| \leq \delta
$$

Proof of Proposition 5.1 This follows from Proposition 4.5 given an $r$-independent, bound on the function $\underline{M}$. The Sections 5.a-5.d explain how the required bound is obtained.

\section{5.a The functions $\underline{M}$ and $\underline{E}$}

Let $\mathfrak{d}=(A, \psi): \mathbb{R} \rightarrow \operatorname{Conn}(E) \times \mathbb{C}^{\infty}(M ; \mathbb{S})$ denote an instanton solution to (1-11). Introduce $\mathrm{E}(s)$ to denote $\mathrm{E}\left(\left.A\right|_{s}\right)$ and introduce

$$
\underline{\mathrm{E}}(s)=\int_{s}^{s+1} \mathrm{E}(x) d x .
$$

This section explains how bounds on $\underline{\mathrm{E}}$ give bounds on $\underline{\mathrm{M}}$.

To start this task, use the top equation in (1-11) with an integration by parts to see that

$$
\frac{d}{d s} \mathrm{E}=-2 \mathrm{E}+2 \mathrm{~L}
$$

where

$$
\mathrm{L}(s)=r \int_{\{s\} \times M}\left(1-|\alpha|^{2}+|\beta|^{2}\right)+\int_{M} a \wedge *\left(\mu+\frac{1}{2} B_{A_{K}}\right) .
$$


Integrate (5-2) to see that

$$
\mathrm{E}(s)=e^{-2 s} \int_{-\infty}^{s} e^{2 x} \mathrm{~L}(x) d x
$$

It follows from Lemma 3.1 that $\mathrm{L}(s) \geq-c_{0}$; and thus (5-4) implies the bound

$$
c_{0} \leq \mathrm{E}(s) \leq e^{2 t}\left(\mathrm{E}(s+t)+c_{0}\right) .
$$

Now define $\underline{\mathrm{L}}(s)$ to be $\int_{s}^{s+1} \mathrm{~L}(x) d x$. It then follows from (5-4) and (5-5) that

$$
\mathrm{E}(s) \leq c_{0}+c_{0} \underline{\mathrm{E}}(s+1) \quad \text { and } \quad \underline{\mathrm{L}}(s) \leq c_{0} \underline{\mathrm{E}}(s)+c_{0} \underline{\mathrm{E}}(s+2)+c_{0} .
$$

Note in particular that a bound on $\underline{E}$ supplies a bound on $\underline{\mathrm{L}}$. Meanwhile, it is a consequence of Lemma 3.1 that $|\underline{\mathrm{M}}-\underline{\mathrm{L}}| \leq c_{0}$. Thus, a bound on $\underline{\mathrm{E}}$ gives a bound on $\underline{\mathrm{M}}$. Granted all of this, the next two lemmas supply a bound on $\underline{\mathrm{E}}$ and hence a bound on $\underline{\mathrm{M}}$. These lemmas again use $\mathrm{E}_{+}$to denote the $s \rightarrow \infty$ limit of $\mathrm{E}\left(\left.A\right|_{s}\right)$.

Lemma 5.2 Assume that $c_{1}(\operatorname{det}(\mathbb{S}))$ is torsion. Given $\mathcal{K} \geq 1$, there exists $\kappa \geq 1$ with the following significance: Suppose that $r \geq \kappa$ and that $\mathfrak{d}=(A, \psi)$ is an instanton solution to (1-11) with $\mathrm{A}_{\mathfrak{d}} \leq \mathcal{K} r$ or $f_{\mathfrak{d}}>-\kappa^{-1} r^{2}$. Assume that $\mathrm{E}_{+} \leq \mathcal{K}$. Then $\sup _{s \in \mathbb{R}} \underline{E}(s) \leq \kappa \mathcal{K}+\kappa^{2}$.

Lemma 5.3 Assume that $c_{1}(\operatorname{det}(\mathbb{S}))$ is not torsion. Given $\mathcal{K} \geq 1$, there is a constant $\kappa \geq 1$ with the following significance: Suppose that $r \geq \kappa$ and that $\mathfrak{d}=(A, \psi)$ is an instanton solution to (1-11) with $\mathrm{A}_{\mathfrak{d}} \leq \mathcal{K} r$ or $f_{\mathfrak{d}} \geq-\mathcal{K} r$ and $\mathrm{E}_{+} \leq \mathcal{K}$. Then $\sup _{s \in \mathbb{R}} \underline{\mathrm{E}}(s) \leq \kappa \mathcal{K}$.

The remaining subsections supply the proofs of these last two lemmas.

\section{5.b Preliminaries}

Given $s \in \mathbb{R}$, let $\mathfrak{a}(s)=\mathfrak{a}\left(\left.\mathfrak{d}\right|_{s}\right)$ denote the value of (3-2) on $\left.\mathfrak{d}\right|_{s}$. Bounds on $\mathfrak{a}(s)$ are obtained here in terms of $\mathrm{E}(s), \mathrm{L}(s)$ and

$$
\mathrm{O}(s)=\int_{\{s\} \times M}\left(\left|\mathfrak{B}_{(A, \psi)}\right|^{2}+r\left|D_{A} \psi\right|^{2}\right) .
$$

Here, $\mathfrak{B}$ is defined in (3-4). The bounds for the various terms in $\mathfrak{a}(s)$ are obtained sequentially moving from right (easy) to left (hard).

The right most term in (3-2) is bounded using Holder's inequality:

$$
r\left|\int_{M} \psi^{\dagger} D_{A} \psi\right| \leq c_{0} r^{1 / 2} \mathrm{O}^{1 / 2} .
$$


The next term in line is bounded by using (3-4) to write $B_{A}$ in terms of $\mathfrak{B}_{(A, \psi)}$ and a remainder. What with Lemma 3.1, an application of Holder's inequality to the resulting expression finds

$$
\left|\mathfrak{e}_{\mu}\right| \leq c_{0}+c_{0}\left(\mathrm{~L}+\mathrm{O}^{1 / 2}\right) .
$$

The term $-\frac{1}{2} r \mathrm{E}$ is left as is, and so all that remains is to bound $\frac{1}{2} \mathfrak{c s}$. Suppose that $u: M \rightarrow U(1)$ is a given smooth map, and write $A-u^{-1} d u=A_{E}+\widehat{a}$. Let $\mathfrak{c s}_{u}(s)=$ $\mathfrak{c s}\left(A_{u}\right)$. Introduce $\hat{a}^{\perp}$ to denote the $L^{2}$ orthogonal projection of $\hat{a}$ onto the subspace of $i \mathbb{R}$-valued coclosed 1 -forms on $M$ that are $L^{2}$ orthogonal to the space of $i \mathbb{R}$ valued harmonic 1 -forms on $M$. Then

$$
\int_{M} \hat{a} \wedge * d \hat{a}=\int_{M} \hat{a}^{\perp} \wedge * d \hat{a}^{\perp} .
$$

The operator $* d$ on $C^{\infty}\left(M ; i T^{*} M\right)$ is invertible on the coclosed 1 -forms that are $L^{2}$ orthogonal to the harmonic 1 -forms. Let $\Pi^{\perp}$ denote the $L^{2}$-orthogonal projection onto the latter space. Use $h_{\mathfrak{B}}$ and $h_{L}$ to denote the respective elements in $\Pi^{\perp} C^{\infty}\left(M ; i T^{*} M\right)$ that obey $* d h_{\mathfrak{B}}=\Pi^{\perp} \mathfrak{B}_{(A, \psi)}$ and $* d h_{L}=r \Pi^{\perp}\left(\psi^{\dagger} \tau \psi-i a\right)$. Thus,

$$
\hat{a}^{\perp}=h_{\mathfrak{B}}+h_{L}+i \mu \text {. }
$$

It follows from Lemma 3.1 that

$$
\left|h_{L}\right| \leq c_{0}\left(r^{1 / 3}|\mathrm{~L}|^{2 / 3}+1\right) .
$$

The proof of this is essentially identical to the proof of the third bullet of [8, Proposition 4.10]. Meanwhile, the $L^{2}$ norm of $h_{\mathfrak{B}}$ on $M$ is bounded by $c_{0} \mathrm{O}^{1 / 2}$. All of this being the case, it follows using (5-10) that

$$
\left|\int_{M} \hat{a} \wedge * d \hat{a}\right| \leq c_{0}\left(1+\mathrm{O}+r^{2 / 3}|\mathrm{~L}|^{4 / 3}\right) .
$$

The term $\hat{a}-\widehat{a}^{\perp}$ contributes to $\mathfrak{c s}$ only via the right most term in (3-3). The latter is proportional to the value on the Poincaré dual of $c_{1}(\operatorname{det}(\mathbb{S}))$ of the cohomology class defined by the $L^{2}$ orthogonal projection of $\hat{a}$ onto the space of harmonic 1 -forms, thus to $\mathfrak{p}(\hat{a})$.

Let $\mathfrak{a}_{\mathfrak{u}}(s)=\mathfrak{a}\left(A-u^{-1} d u, u \psi\right)$. Putting all that was just said together gives

$$
\mathfrak{a}_{\mathfrak{u}}(s) \leq c_{0}\left(1+\mathrm{L}+r^{1 / 2} \mathrm{O}^{1 / 2}+\mathrm{O}+r^{2 / 3}|\mathrm{~L}|^{4 / 3}\right)+i \pi \mathfrak{p}(\widehat{a})-\frac{1}{2} r \mathrm{E} .
$$


Since $\mathfrak{a}_{\mathfrak{u}}(s)-\mathfrak{a}_{\mathfrak{u}}\left(s^{\prime}\right)$ is independent of $u$, it follows from Lemma 3.4 that the function $s \rightarrow \mathfrak{a}_{\mathfrak{u}}(s)$ is a nonincreasing function on $\mathbb{R}$. As a consequence, (5-14) finds the bound

$$
r \mathrm{E} \leq-2 \mathfrak{a}\left(u \mathfrak{c}_{+}\right)+c_{0}\left(r+\mathrm{L}+\mathrm{O}+r^{2 / 3}|\mathrm{~L}|^{4 / 3}\right)+i \pi \mathfrak{p}(\widehat{a}) .
$$

Here, $u \mathfrak{c}_{+}$is used to denote the gauge transform by $u$ of the configuration $\mathfrak{c}_{+}$.

To exploit this, introduce $\underline{\mathrm{O}}(s)$ to denote $\int_{s}^{s+1} \mathrm{O}(x) d x$. The measure of the subset in $[s, s+1]$ where $\mathrm{O} \geq 8 \underline{\mathrm{O}}$ is no greater than $\frac{1}{8}$. By the same token, the measure of the subset in $[s, s+1]$ where $\mathrm{L} \geq 8 \underline{\mathrm{L}}$ is no greater than $\frac{1}{8}$. Thus, there exist points $s^{\prime} \in[s, s+1]$ where both $\mathrm{O} \leq 8 \underline{\mathrm{O}}$ and $\mathrm{L} \leq 8 \underline{\mathrm{L}}$. Take such a point in $(5-15)$ to see that

$$
r \mathrm{E}\left(s^{\prime}\right) \leq-2 \mathfrak{a}\left(u \mathfrak{c}_{+}\right)+c_{0}\left(r+\underline{\mathrm{L}}+\underline{\mathrm{O}}+r^{2 / 3}|\underline{\mathrm{L}}|^{4 / 3}\right)+i \pi \mathfrak{p}\left(\left.\hat{a}\right|_{s^{\prime}}\right) .
$$

Now use the right hand inequality in (5-4) to see that $\mathrm{E}(x) \leq c_{0} \mathrm{E}\left(s^{\prime}\right)+c_{0}$ for all $x \in[s-1, s]$. This understood, (5-6) and (5-16) give

$$
r \underline{\mathrm{E}}(s-1) \leq-c_{0} \mathfrak{a}\left(u \mathfrak{c}_{+}\right)+c_{0}(r+\underline{\mathrm{O}}(s))
$$

$$
+c_{0} r^{2 / 3} \sup _{x \geq s}|\underline{E}(x)|^{4 / 3}+c_{0} \sup _{[s, s+1]}|\mathfrak{p}(\widehat{a})| .
$$

Finally, use the second equality in Lemma 3.4 to bound $\underline{\mathrm{O}}(s)$ and so find that

$$
\begin{aligned}
& r \underline{\mathrm{E}}(s-1) \leq-c_{0} \mathfrak{a}\left(u \mathfrak{c}_{+}\right)+c_{0}\left(r+\mathfrak{a}\left(\mathfrak{c}_{-}\right)-\mathfrak{a}\left(\mathfrak{c}_{+}\right)\right) \\
& +c_{0} r^{2 / 3} \sup _{x \geq s}|\underline{\mathrm{E}}(x)|^{4 / 3}+c_{0} \sup _{[s, s+1]}|\mathfrak{p}(\widehat{a})| .
\end{aligned}
$$

\section{5.c Proof of Lemma 5.2}

Since $\mathrm{E} \leq c_{0}+c_{0} r$ in any event, it is sufficient to consider the case where $\mathrm{E}_{+} \leq \varepsilon r$ for a suitable $\varepsilon>c_{0}^{-1}$ This understood, suppose that $\varepsilon>0$ has been fixed and assume in what follows the bound $\mathrm{E}_{+} \leq \varepsilon r$.

Given that $c_{1}(\operatorname{det}(\mathbb{S}))$ is a torsion class, the term with $\mathfrak{p}(\widehat{a})$ is absent in (5-18) and $\mathfrak{a}\left(u \mathfrak{c}_{+}\right)=\mathfrak{a}\left(\mathfrak{c}_{+}\right)$. To bound $-\mathfrak{a}\left(\mathfrak{c}_{+}\right)$in $(5-18)$, use the fact that $\left|\mathfrak{a}\left(\mathfrak{c}_{+}\right)+\frac{1}{2} r \mathrm{E}_{+}\right|$is equal to $\left|\frac{1}{2} \mathfrak{c s}\left(\mathfrak{c}_{+}\right)+\mathfrak{e}_{\mu}\right|$, and so it follows from (5-19) that $\left|\mathfrak{a}\left(\mathfrak{c}_{+}\right)\right| \leq c_{0} r \mathrm{E}_{+}$.

To continue, consider the term $\mathfrak{a}\left(\mathfrak{c}_{-}\right)-\mathfrak{a}\left(\mathfrak{c}_{+}\right)$. This is $A_{\mathfrak{d}}$, and so this difference is bounded by default by $\mathcal{K} r$ if the apriori assumption on $A_{\mathfrak{d}}$ is satisfied. As explained next, a bound on this difference by $c_{0} \mathcal{K} r$ also follows if $f_{\mathfrak{d}}>-c_{0}^{-1} r^{2}$. To see why, use the bound on $\left|\mathfrak{a}\left(\mathfrak{c}_{+}\right)\right|$in the preceding paragraph. If $\mathfrak{a}\left(\mathfrak{c}_{-}\right)<0$, it can be discarded from (5-18). Suppose that $\mathfrak{a}\left(\mathfrak{c}_{-}\right) \geq 0$. This condition requires that $\mathfrak{c s}\left(\mathfrak{c}_{-}\right) \geq r \mathrm{E}\left(\mathfrak{c}_{-}\right)-c_{0}$. However, according to [8, Proposition 4.10], if $c_{1}(\operatorname{det}(\mathbb{S}))$ is torsion, then

$$
c_{0}\left(r^{2 / 3}|\mathrm{E}|^{4 / 3}+c_{0}\right) \geq|\mathfrak{c} \mathfrak{s}|,
$$


for any solution to (1-8). Applied to $\mathfrak{c}_{-}$, this inequality together with the condition that $\mathfrak{c s}\left(\mathfrak{c}_{-}\right) \geq r \mathrm{E}\left(\mathfrak{c}_{-}\right)-c_{0}$ leads to the following dichotomy: Fix $c>c_{0}$. Either $\mathrm{E}\left(\mathfrak{c}_{-}\right) \leq c r^{-1}$, in which case $\mathfrak{c s}\left(\mathfrak{c}_{-}\right) \leq c_{0}$ when $r$ is large, and thus $\mathfrak{a}\left(\mathfrak{c}_{-}\right) \leq c_{0}$; or else $\mathrm{E}\left(\mathfrak{c}_{-}\right) \geq c r^{-1}$. In the latter case, (5-19) together with the condition $\mathfrak{c s} \geq r \mathrm{E}-c_{0}$ requires that $\mathfrak{c s}\left(\mathfrak{c}_{-}\right) \geq c_{0}^{-1} r^{2}$ if $c \geq c_{0}$. However, this is not possible if $\varepsilon \leq c_{0}^{-1}$ given the bound $f_{\mathfrak{d}} \geq-c_{0}^{-1} r^{2}$. Indeed, with [7, Proposition 5.1], the condition $\mathfrak{c s}\left(\mathfrak{c}_{-}\right) \geq c_{0}^{-1} r^{2}$ requires that the spectral flow function on $\mathfrak{c}_{-}$be greater than $c_{0}^{-1} r^{2}$. Meanwhile, (5-19) applied to $\mathfrak{c}_{+}$implies that $\left|\mathfrak{c s}\left(\mathfrak{c}_{+}\right)\right| \leq c_{0} \varepsilon^{4 / 3} r^{2}$ because $\mathrm{E}_{+} \leq \varepsilon r$. This understood, [7, Proposition 5.1] bounds the absolute value of the spectral flow function on $\mathfrak{c}_{+}$from below by $-c_{0} \varepsilon^{4 / 3} r^{2}$. Thus, the spectral flow $f_{\delta}$ would be less than $-c_{0}^{-1} r^{2}$ were $\varepsilon \leq c_{0}^{-1}$. Hence, if $\varepsilon \leq c_{0}^{-1}$ and $\mathrm{E}_{+} \leq \varepsilon r$, then $\mathfrak{a}\left(\mathfrak{c}_{-}\right) \leq c_{0} r^{2 / 3}$.

Granted the preceding, (5-18) implies that

$$
\underline{\mathrm{E}}(s-1) \leq c_{0}(1+\mathcal{K})+c_{0} r^{-1 / 3} \sup _{x \geq s}|\underline{\mathrm{E}}(x)|^{4 / 3} .
$$

Given $T \geq 1$, use $s_{T}$ to denote the largest value of $s \in \mathbb{R}$ where $\underline{\mathrm{E}}(s-1) \geq \mathcal{K}+T$. Take $s=s_{T}$ in (5-20) to see that

$$
T \leq c_{0}+c_{0} \mathcal{K}+c_{0} r^{-1 / 3} T^{4 / 3} .
$$

Given that $\mathrm{E}_{+} \leq \varepsilon r$, it follows from (5-21) that there exist $(A, \psi)$ and $r$-independent constants $z_{0} \geq 1$ and $z_{1} \geq 1$ with the following property: If $\varepsilon \leq c_{0}^{-1}$, then

$$
T=z_{0}+z_{1} \mathcal{K}
$$

can not be a solution to (5-21). This last observation implies Lemma 5.2's claim.

\section{5.d Proof of Lemma 5.3}

The argument for this lemma has three parts.

Part 1 Consider the version of (5-18) that arises when the map $u: M \rightarrow S^{1}$ is chosen as follows: Write $\mathfrak{c}_{+}=\left(A_{+}, \psi_{+}\right)$. Take $u$ so that $A_{+}-u^{-1} d u=A_{E}+\widehat{a}_{+}$where $\hat{a}_{+}$is coclosed and where the $L^{2}$ norm of its projection into the space of harmonic 1 -forms is bounded by 100 . Such a choice for $u$ is always available. Granted this choice, it follows using [7, Lemma 2.4, (4-2)] that

$$
\left|\mathfrak{a}\left(u \mathfrak{c}_{+}\right)+\frac{1}{2} r \mathrm{E}_{+}\right|=\left|\frac{1}{2} \mathfrak{c s}+\mathfrak{e}_{\mu}\right| \leq c_{0}+c_{0} r \mathrm{E}_{+} .
$$

If the assumption on $A_{\mathfrak{d}}$ is obeyed, replace $\mathfrak{a}\left(\mathfrak{c}_{-}\right)-\mathfrak{a}\left(\mathfrak{c}_{+}\right)$in (5-18) by $\mathcal{K} r$. If this assumption is not obeyed, introduce the function $\mathfrak{a}^{f}=a-2 \pi^{2} f$ on $\operatorname{Conn}(E) \times \mathbb{C}^{\infty}(M ; \mathbb{S})$. This function is constant on the $C^{\infty}(M ; U(1))$ orbits in $\operatorname{Conn}(E) \times \mathbb{C}^{\infty}(M ; \mathbb{S})$, 
although only defined at those pairs where (1-14) has trivial kernel. Such is the case for $\mathfrak{c}_{-}$and $\mathfrak{c}_{+}$. Moreover,

$$
\mathfrak{a}^{f}\left(\mathfrak{c}_{-}\right)-\mathfrak{a}^{f}\left(\mathfrak{c}_{+}\right)=\mathfrak{a}\left(\mathfrak{c}_{-}\right)-\mathfrak{a}\left(\mathfrak{c}_{+}\right)+2 \pi^{2} f_{\mathfrak{d}}
$$

This is used in (5-18) to replace $\mathfrak{a}\left(\mathfrak{c}_{-}\right)-\mathfrak{a}\left(\mathfrak{c}_{+}\right)$by $\mathfrak{a}^{f}\left(\mathfrak{c}_{-}\right)-\mathfrak{a}^{f}\left(\mathfrak{c}_{+}\right)+c_{0} \mathcal{K} r$ if the assumption on $A_{\mathfrak{d}}$ is not obeyed. To continue in this case, discard $\mathfrak{a}^{f}\left(\mathfrak{c}_{-}\right)$if the latter is nonpositive. As argued next, it is always the case $\mathfrak{a}^{f}\left(\mathfrak{c}_{-}\right) \leq c_{0}$ when $f_{\mathfrak{d}} \geq-\mathcal{K} r$ and $r$ is large. To see why, suppose that $\mathfrak{a}^{f}\left(\mathfrak{c}_{-}\right) \geq 0$. This requires that $\mathfrak{c s}^{f}\left(\mathfrak{c}_{-}\right) \geq r \mathrm{E}\left(\mathfrak{c}_{-}\right)-c_{0}$ with $\mathfrak{c s}^{f}$ denoting $\mathfrak{c s}-4 \pi^{2} f$. Meanwhile [8, Proposition 4.10] asserts that

$$
\left|\mathfrak{c s}^{f}\right| \leq c_{0}\left(r^{2 / 3}|E|^{4 / 3}(\ln r)^{c_{0}}+1\right)
$$

for any solution to (1-8).

Fix $c \geq c_{0}$ and there is the following dichotomy: Either $\mathrm{E}\left(\mathfrak{c}_{-}\right) \leq c r^{-1}$, in which case (5-25) finds $\left|\mathfrak{c s}^{f}\left(\mathfrak{c}_{-}\right)\right| \leq c_{0}$ and thus $\mathfrak{a}^{f}\left(\mathfrak{c}_{-}\right) \leq c_{0}$; or else $\mathrm{E}\left(\mathfrak{c}_{-}\right) \geq c r^{-1}$. If $c \geq c_{0}$, this with (5-25) and the lower bound $\mathfrak{c s}^{f}\left(\mathfrak{c}_{-}\right) \geq r \mathrm{E}\left(\mathfrak{c}_{-}\right)-c_{0}$ requires $\mathrm{E}\left(\mathfrak{c}_{-}\right) \geq c_{0} r(\operatorname{lnr})^{-c_{0}}$; and therefore the upper bound $\left|\mathfrak{c s}^{f}\right| \geq c_{0} r^{2}(\operatorname{lnr})^{-c_{0}}$. But, this violates [7, Proposition 5.1] which finds the apriori bound $\left|\mathfrak{c s}^{f}\right| \leq c_{0} r^{31 / 16}$. To summarize, $\mathfrak{a}^{f}\left(\mathfrak{c}_{-}\right) \leq c_{0}$ in the case $f_{\mathfrak{d}} \geq-\mathcal{K} r$. Meanwhile, (5-25) applied to $\mathfrak{c}_{+}$bounds $\left|\mathfrak{c s}^{f}\left(\mathfrak{c}_{+}\right)\right|$and thus $\left|\mathfrak{a}^{f}\left(\mathfrak{c}_{+}\right)\right|$by $c_{0}+c_{0} \mathrm{E}_{+}$.

Granted all of the above, it follows directly from (5-18) that

$$
\underline{\mathrm{E}}(s-1) \leq c_{0}+c_{0} \mathcal{K}+c_{0} r^{-1 / 3} \sup _{x \geq s}|\underline{\mathrm{E}}(x)|^{4 / 3}+c_{0} r^{-1} \sup _{[s, s+1]}|\mathfrak{p}(\widehat{a})| .
$$

To exploit (5-26), fix $T \geq 1$, and introduce $s_{T}$ to denote the largest value of $s \in \mathbb{R}$ where $\underline{\mathrm{E}}(s-1)>\mathcal{K}+T$. Take $s=s_{T}$ in (5-26) to see that

$$
T \leq c_{0}+c_{0} \mathcal{K}+c_{0} r^{-1 / 3} T^{4 / 3}+c_{0} r^{-1} \sup _{\left[s_{T}, s_{T}+1\right]}|\mathfrak{p}(\widehat{a})| .
$$

Suppose that $|\mathfrak{p}(\hat{a})|$ for $s \in s_{T}+1$ is bounded by $\frac{1}{100} c_{0}^{-1} r(\mathcal{K}+T)$. If such is the case, then (5-27) implies that

$$
T \leq c_{0}\left(1+\mathcal{K}+r^{-1 / 3} T^{4 / 3}\right)
$$

this an equation with no solution with $T \geq 2 c_{0}(1+\mathcal{K})$ but less than $c_{0}^{-1} r$. Thus, a bound on $|\mathfrak{p}(\hat{a})|$ for $s \in\left[s_{T}, s_{T}+1\right]$ by a very small multiple of $r(\mathcal{K}+T)$ ) leads to the desired bound on $\sup _{s \in \mathbb{R}} \underline{E}(s)$. As explained in what follows, $|\mathfrak{p}(\widehat{a})|$ has, in fact, an $r$-independent upper bound. 
Part 2 Introduce for each $s \in \mathbb{R}$ the function

$$
\mathrm{M}(s)=r \int_{\{s\} \times M}\left(1-|\alpha|^{2}\right) .
$$

Thus, the integral of $\mathrm{M}(\cdot)$ on any given interval $[s, s+1]$ is the function $\underline{\mathrm{M}}(s)$. It is a consequence of what is written in the first item of Lemma 3.1 that $\mathrm{M}(\cdot) \geq-c_{0}$.

Write $A=A_{E}+\widehat{a}$ as before and introduce

$$
\widehat{a}^{F}=\widehat{a}-\frac{1}{2}\left(|\alpha|^{2}+\chi(|\alpha|)\right)^{-1}\left(\bar{\alpha} \nabla_{A} \alpha-\alpha \nabla_{A} \bar{\alpha}\right)
$$

The connection $A^{F}=A_{E}+\hat{a}^{F}$ is flat where $|\alpha| \geq \frac{1}{2}$ for here $\alpha /|\alpha|$ is $A^{F}$-covariantly constant. Suppose that $\sigma \subset M$ and $\sigma^{\prime}$ are embedded, oriented, homologous loops. Suppose in addition that $s>s^{\prime}$ are points in $\mathbb{R}$ and that $\Sigma \subset\left[s, s^{\prime}\right] \times M$ is a closed immersed surface with respective boundaries $\{s\} \times \sigma$ and $\left\{s^{\prime}\right\} \times \sigma^{\prime}$. Then

$$
\int_{\{s\} \times \sigma} \hat{a}^{F}-\int_{\{s\} \times \sigma^{\prime}} \hat{a}^{F}=0
$$

unless $\Sigma$ enters the region where $|\alpha| \leq \frac{1}{2}$.

In what follows, $c_{T} \geq 1$ denotes a constant that depends on $T$ and $\mathcal{K}$, but not on $r$ and not on $\mathfrak{d}=(A, \psi)$. This understood, fix $r \geq c_{T}$ where $c_{T} \geq 1$ is large enough to invoke Lemmas 3.1, 3.6, 3.8, 3.9 and 3.10 on the domain $\left[s+8, s^{\prime}-8\right] \times M$ using $100(\mathcal{K}+T)$ in lieu of $\mathcal{K}$. Assume $\underline{\mathrm{M}}(\cdot) \leq 100(\mathcal{K}+T)$ on $\left[s+8, s^{\prime}-8\right]$. Suppose that $d>c_{0} r^{-1 / 4}$ and that each point of $\Sigma$ has distance at least $d$ from where $|\alpha| \leq \frac{1}{2}$. This being the case, it follows using Lemma 3.8 and Lemma 3.9 that $\hat{a}^{F}$ can be written on both $\sigma$ and $\sigma^{\prime}$ as

$$
\widehat{a}^{F}=b+\mathfrak{q}
$$

where $|b| \leq\left(c_{T} M(s)+c_{T}\right) d^{-2}$ and where $\mathfrak{q}$ is the projection of $\hat{a}$ onto the space of harmonic 1-forms. Given the bound on $|b|$, what is written in (5-31) implies that

$$
\left|\int_{\{s\} \times \sigma} \mathfrak{q}-\int_{\{s\} \times \sigma^{\prime}} \mathfrak{q}\right| \leq c_{T}\left(\left(\mathrm{M}(s)+\mathrm{M}\left(s^{\prime}\right)\right)+c_{T}\right) \ell d^{-2},
$$

where $\ell=\max \left\{\right.$ length $(\sigma)$, length $\left.\left(\sigma^{\prime}\right)\right\}$. If the homology class of $\sigma$ and $\sigma^{\prime}$ is Poincaré dual to $c_{1}(\operatorname{det}(\mathbb{S}))$, then (5-33) implies that

$$
\left|\mathfrak{p}\left(\left.\widehat{a}\right|_{s}\right)-\mathfrak{p}\left(\left.\widehat{a}\right|_{s^{\prime}}\right)\right| \leq c_{T}\left(\left(\mathrm{M}(s)+\mathrm{M}\left(s^{\prime}\right)\right)+c_{T}\right) \ell d^{-2} .
$$

Given the top line in (1-11), use of Lemma 3.1 and Lemma 3.9 finds that

$$
\left|\frac{d}{d s} \mathfrak{p}(\widehat{a})\right| \leq c_{T}+c_{T} \mathrm{M}(s)
$$


Suppose that $s-s^{\prime} \geq 3$ and that $\Sigma$ intersects $\left[s^{\prime}, s^{\prime}+1\right] \times M$ as $\left[s^{\prime}, s^{\prime}+1\right] \times \sigma^{\prime}$ and has the analogous product structure in $[s-1, s] \times M$. Granted (5-35) and this product structure, then both appearances of $\mathrm{M}(\cdot)$ in (5-34) can be replaced by $\underline{\mathrm{M}}(\cdot)$. In particular, if $s^{\prime}$ is greater than $s_{T}$, then the fact that $|\underline{\mathrm{L}}-\underline{\mathrm{M}}| \leq c_{0}$ together with (5-6) and the $\underline{\mathrm{M}}$ version of (5-34) imply that

$$
\left|\mathfrak{p}\left(\left.\widehat{a}\right|_{s}\right)-\mathfrak{p}\left(\left.\widehat{a}\right|_{s^{\prime}}\right)\right| \leq c_{T}(1+\mathcal{K}+T) \ell d^{-2} .
$$

Keep in mind that this holds if there is an immersed surface $\Sigma \subset\left[s, s^{\prime}\right] \times M$ with distance $d \geq c_{0} r^{-1 / 4}$ from $\alpha^{-1}(0)$, and $\Sigma$ is $\left[s^{\prime}, s^{\prime}+1\right] \times \sigma^{\prime}$ and $[s-1, s] \times \sigma$ at distances 1 or less from the boundaries of $\left[s, s^{\prime}\right] \times M$.

Part 3 This part starts with a lemma that is well known to the pseudoholomorphic curve experts. It is a corollary of Lemma 4.8.

Lemma 5.4 Suppose that $\mathcal{N} \geq 1$ has been given and that each Reeb orbit of length at most $\mathcal{N}$ is nondegenerate. Given also $\delta>0$, there is a constant $\kappa \geq 1$ with the following significance: Suppose that $C \subset \mathbb{R} \times M$ is a pseudoholomorphic subvariety with both $\int_{\mathbb{C}} d a \leq \mathcal{N}$ and $\sup _{s \in \mathbb{R}} \int_{C \cap(I[s, s+1] \times M)} d s \wedge a \leq \mathcal{N}$. Then there is a set $\mathbb{I} \subset \mathbb{R}$ with at most $\kappa$ components and total length $\kappa^{-1}$ or less, and such that if $s \in \mathbb{R}-\mathbb{I}$, then each point of $\left.C\right|_{s}$ has distance $\delta$ or less from a Reeb orbit in $M$ with length at most $\mathcal{N}$.

Keep this lemma in mind.

Fix $\varepsilon>0$. With $T$ fixed, there exists $K_{T, \varepsilon} \geq 1$ such that if $r \geq \kappa_{T, \varepsilon}$, then Proposition 4.5 can be invoked using $\mathcal{K}+T$ in lieu of $\mathcal{K}$, using $\varepsilon$ in lieu of $\delta$, and using for $I$ the set $\left[s_{T}+16, \infty\right)$. The proposition supplies a constant $N_{T}$ and some $N \leq N_{T}$ intervals $\left\{I_{k}\right\}_{k=1, \ldots, N}$ and corresponding sets $\left\{\vartheta_{k}\right\}_{k=1, \ldots, N}$. Granted what is said about these sets in Proposition 4.5, and granted Lemma 5.4, there exist $N_{T \varepsilon}, L_{T, \varepsilon} \geq 1$ and there exists a closed set $\mathbb{I}^{\prime} \subset\left[s_{T}+16, \infty\right)$ composed of $N \leq N_{T, \varepsilon}$ disjoint intervals of length at most $L_{T, \varepsilon}$ such that the following is true: If $s \in\left[s_{T}+16, \infty\right)$ is not in $\mathbb{I}^{\prime}$, then each point of $\left.\alpha^{-1}(0)\right|_{s}$ has distance $\varepsilon$ or less from a Reeb orbit with length at most $(1 /(2 \pi)) \mathrm{E}_{+}$.

Given the preceding, fix a smooth embedded loop in $M$ whose points have distance at least $4 \varepsilon$ from each Reeb orbit with length at most $(1 /(2 \pi)) \mathrm{E}_{+}$. Choose this loop so that its homology class is Poincaré dual to $c_{1}(\operatorname{det}(\mathbb{S}))$. Knowing that the Reeb orbits are nondegenerate, there exists $\varepsilon_{T}>0$ with the following significance: If $\varepsilon<\varepsilon_{T}$, then there exists such a loop with length bounded by a $T$-dependent constant, $\ell_{T}$. This understood, take $\varepsilon=\varepsilon_{T}$ and fix such a loop, $\sigma$, as just described. Now consider (5-35) with $d=\varepsilon_{T}$, with $\left[s, s^{\prime}\right] \subset\left[s_{T}+16, \infty\right)-I^{\prime}$ and with $\Sigma=\left[s, s^{\prime}\right] \times \sigma$. This 
version of (5-35) bounds the total change in $\mathfrak{p}(\widehat{a})$ on any such $\left[s, s^{\prime}\right]$. In particular, it implies that the total change in $\mathfrak{p}(\widehat{a})$ over any component of $\left[s_{T}+16, \infty\right)-\mathbb{I}^{\prime}$ is bounded by $c_{T}(\mathcal{K}+T) \ell_{T} \varepsilon_{T}^{-2}$. Note that there are no more than $N_{T, \varepsilon}+1$ components of $\left[s_{T}+16, \infty\right)-\mathbb{I}^{\prime}$. Meanwhile, what with (5-34), the total change in $\mathfrak{p}(\widehat{a})$ over any component of $\mathbb{I}^{\prime}$ and over $\left[s_{T}, s_{T}+16\right]$ is at most $c_{T} L_{T, \varepsilon}(\mathcal{K}+T)$. As there are at most $N_{T, \varepsilon}+1$ such components, the total change in $\mathfrak{p}(\widehat{a})$ between any $s \in\left[s_{T}, \infty\right)$ and $\infty$ is at most

$$
c_{T}(\mathcal{K}+T) N_{T, \varepsilon} L_{T, \varepsilon} \ell_{T} \varepsilon_{T}^{-2} .
$$

Granted (5-37), then (5-27) leads to (5-28) if $r$ is sufficiently large, and thus a uniform bound on $T$ by $c_{0} \mathcal{K}$.

\section{5.e More about limits}

The proposition that follows strengthens Proposition 5.1 as it asserts that all curves in each $k \in\{1, \ldots, N\}$ version of $\vartheta_{k}$ are defined on the whole of $\mathbb{R} \times M$. As in Proposition 5.1, the pair $(a, J)$ consist of a contact 1 -form on $M$ and a compatible almost complex structure on the kernel of $a$. The latter with a given choice of $\mu$ with $\mathcal{P}$-norm less than 1 are used to define (1-11).

Proposition 5.5 Fix $(a, J)$ as above. Given $\mathcal{K} \geq 1$ and $\mathrm{E}_{+} \leq \mathcal{K}$, there exists $\kappa \geq 1$, and given $\delta>0$, there exists $\kappa_{\delta} \geq 1$ which, with $\kappa$, has the following significance: Suppose that $r \geq \kappa_{\delta}$, and that $\mathfrak{d}=(A, \psi=(\alpha, \beta))$ is an instanton solution to (1-11). If $c_{1}(\operatorname{det}(\mathbb{S}))$ is a torsion class, assume $\mathrm{A}_{\mathfrak{d}} \leq \mathcal{K} r$ or $f_{\mathfrak{d}} \geq-\kappa^{-1} r^{2}$. If $c_{1}(\operatorname{det}(\mathbb{S}))$ is not a torsion class, assume that $\mathrm{A}_{\mathfrak{d}} \leq \mathcal{K} r$ or that $f_{\mathfrak{d}} \geq-\mathcal{K} r$. In either case, assume that $\mathrm{E}_{+}=\lim _{s \rightarrow \infty} \mathrm{E}\left(\left.A\right|_{s}\right) \leq \mathcal{K}$ and that all Reeb orbits of length no greater than $(1 /(2 \pi)) \mathrm{E}_{+}$are nondegenerate.

- Let $\mathfrak{c}_{-}=\left.\lim _{s \rightarrow-\infty}(A, \psi)\right|_{s}$. Then $\mathfrak{c}_{-}$is a solution to $(1-11)$ with $\mathrm{E}\left(\mathfrak{c}_{-}\right) \leq$ $\mathrm{E}_{+}+\delta$.

- Each point in $\mathbb{R} \times M$ where $|\alpha| \leq 1-\delta$ has distance at most $\kappa r^{-1 / 2}$ from where $\alpha=0$.

- Moreover, there exists

(a) A positive integer $N \leq \kappa$ and a cover of $\mathbb{R}$ as $\bigcup_{1 \leq k \leq N} I_{k}$ by connected open sets of length at least $2 \delta^{-1}$. These are such that $I_{k} \cap I_{k^{\prime}}=\varnothing$ if $\left|k-k^{\prime}\right|>1$. In addition, if $\left|k-k^{\prime}\right|=1$, then $I_{k} \cap I_{k^{\prime}}$ has length between $\frac{1}{128} \delta^{-1}$ and $\frac{1}{64} \delta^{-1}$. 
(b) For each $k \in\{1,2, \ldots, N\}$, a set $\vartheta_{k}$ whose typical element is a pair $(C, m)$ where $m$ is a positive integer and where $C \subset \mathbb{R} \times M$ is a pseudoholomorphic subvariety. These elements of $\vartheta_{k}$ are constrained so that

$$
\sum_{(C, m) \in \vartheta_{k}} m \int_{C} d a<\kappa
$$

In addition, these sets $\left\{\vartheta_{k}\right\}_{k=1, \ldots, N}$ are such that

(1)

$$
\sup _{\substack{z \in \bigcup_{(C, m) \in \vartheta_{k}} C, s(z) \in I_{k}}} \operatorname{dist}\left(z, \alpha^{-1}(0)\right)+\sup _{\substack{z \in \alpha^{-1}(0), s(z) \in I_{k}}} \operatorname{dist}\left(\bigcup_{(C, m) \in \vartheta_{k}} C, z\right)<\delta .
$$

(2) Let $k \in\{1, \ldots, N\}$, let $I^{\prime} \subset I_{k}$ denote an interval of length 1 , and let $v$ denote the restriction to $I^{\prime} \times M$ of a $2-$ form on $\mathbb{R} \times M$ with $\|v\|_{\infty}=1$ and $\|\nabla v\|_{\infty} \leq \delta^{-1}$. Then

$$
\left|\frac{i}{2 \pi} \int_{I \times M} v \wedge F_{\hat{A}}-\sum_{(C, m) \in \vartheta} m \int_{C} v\right| \leq \delta .
$$

Proof of Proposition 5.5 Start with the assertion that each curve in each $k \in$ $\{1, \ldots, N\}$. If this is not true, there would exist an unbounded sequence $\left\{r_{n}\right\}_{n=1,2, \ldots}$ and a corresponding sequence of instantons $\left\{\mathfrak{d}_{n}\right\}_{n=1,2, \ldots}$ to the corresponding $r=r_{j}$ version of (1-11) that obeyed the assumptions with fixed $\mathcal{K}$ and $\delta$, but such that this particular assertion was false. In any event, the conclusions of Proposition 5.1 hold for $n$ sufficiently large. Since Proposition 5.1's integer $N$ is uniformly bounded, a subsequence (hence renumbered consecutively from 1) can be chosen so that all members have the same integer $N$. By passing to another subsequence, one can assume that there exists an integer $k \in\{1, \ldots, N\}$ and a sequence $\left\{\vartheta_{k, n}\right\}_{n=1,2, \ldots}$ where any given index $n$ element comes from some index $j$ version of one of Proposition 5.1's sets $\vartheta_{k}$. Moreover, each such $\vartheta_{k, n}$ contains a pair whose $J$-holomorphic curve is not the restriction to some neighborhood of $I_{k} \times M$ of a $J$-holomorphic subvariety that obeys the Proposition 5.5's requirements. Finally, there is no replacement of $\vartheta_{k, n}$ by a corresponding set that does obey Proposition 5.5's requirements. This last conclusion is seen below to generate nonsense.

The $n$-independent bound on the integral of $d a$ over the constituent curves in any given $\vartheta_{k, n}$ implies that the sequence $\left\{\left\{\vartheta_{k, n}\right\}_{1 \leq k \leq N}\right\}_{n=1,2, \ldots}$ has a subsequence hence renumbered consecutively that converges to what is often called a "broken trajectory" of $J$-holomorphic subvarieties. This broken trajectory consists of a finite, ordered collection $\left\{\vartheta_{k}\right\}_{k=1,2, \ldots, N^{\prime}}$, of sets with the following properties: The typical element in each set is a pair $(C, m)$ where $C \subset \mathbb{R} \times M$ is a pseudoholomorphic subvariety 
and where $m$ is a positive integer. As usual, no two pairs from any given $\vartheta_{k}$ share the same subvariety. Moreover, at least one pair from $\vartheta_{k}$ has a subvariety that is not $\mathbb{R}$-invariant. There are also constraints on the $s \rightarrow \pm \infty$ limits of the pairs from $\vartheta_{k}$.

To say more about these constraints, note that the $s \rightarrow-\infty$ limit of the pairs from $\vartheta_{k}$ defines a set, $\Theta_{k-}$, whose typical element is a pair $(\gamma, m)$ with $\gamma$ a Reeb orbit and $m$ a positive integer. In this regard, $(\gamma, m)$ appears if $\gamma$ is an $s \rightarrow-\infty$ limit of the constant $s$ slices of $\Sigma_{k}=\bigcup_{\left(C, m^{\prime}\right) \in \vartheta_{k}} C$. What follows describes how $m$ is obtained. Let $\mathcal{E}_{\gamma}$ - denote the ends of $\Sigma_{k}$ whose constant $s$ slices converge as $s \rightarrow-\infty$ to $\gamma$. Then $m$ is a sum of positive integers with the sum indexed by the ends in $\mathcal{E}_{\gamma-}$. In particular, an end $\mathcal{E}$ contributes to this sum the product $m_{\mathcal{E}} m^{\prime}$ where $m^{\prime}$ is the integer component of the pair from $\vartheta_{k}$ that contains $\mathcal{E}$ and where $m_{\mathcal{E}}$ is the degree of the projection from any constant $s \ll-1$ slice of $\mathcal{E}$ to $\gamma$. The $s \rightarrow \infty$ limit of the pairs from $\vartheta_{k}$ define an analogous set, this denoted by $\Theta_{k+}$. With this notation in hand, the pairs $\left\{\vartheta_{k}\right\}_{k=1, \ldots, N}$ are such that $\Theta_{k-}=\Theta_{k-1,+}$ for $k>1$.

The convergence of $\left\{\left\{\vartheta_{k, n}\right\}_{k=1, \ldots, N}\right\}_{n=1,2, \ldots}$ to $\left\{\vartheta_{k}\right\}_{k=1,2, \ldots, N^{\prime}}$ is such that the following is true: Let $I_{k, n}$ denote the index $n$ version of Proposition 5.5's interval $I_{k}$. The set of integers $\left\{1, \ldots, N^{\prime}\right\}$ is partitioned into $N$ subsets. These are denoted by $\left\{\Lambda_{k}\right\}_{1 \leq k \leq N}$. The sequence $\left\{\vartheta_{k, n}\right\}_{n=1,2, \ldots}$ determines the collection $\left\{\vartheta_{k^{\prime}}: k^{\prime} \in \Lambda_{k}\right\}$. For each $n$, the interval $I_{k, n}$ is written as a union of consecutive intervals that are indexed by the elements in $\Lambda_{k}$. Each subinterval can be assumed to have length greater than $N$. Let $k^{\prime} \in \Lambda_{k}$ and let $I_{k^{\prime}, n} \subset I_{k, n}$ denote the corresponding subinterval. Let $\phi_{k^{\prime}, n^{\prime}}: \mathbb{R} \times M \rightarrow \mathbb{R} \times M$ denote the constant translation along the $\mathbb{R}$ factor that puts the midpoint of $I_{k^{\prime}, n}$ at the origin.

$$
\begin{aligned}
& \sum_{(C, m) \in \vartheta_{k^{\prime}}} m \int_{C} d a=\lim _{n \rightarrow \infty} \sum_{(C, m) \in \vartheta_{k, n}} m \int_{C \cap I_{k^{\prime}, n}} d a . \\
& \text { - } \quad \sum_{(C, m) \in \vartheta_{k^{\prime}}} m \int_{\mathbb{C}} v=\lim _{n \rightarrow \infty} \sum_{(C, m) \in \vartheta_{k, j}} m \int_{C \cap I_{k^{\prime}, n}} \phi_{k^{\prime}, n} * v,
\end{aligned}
$$

where $v$ denotes a 2 -form on $\mathbb{R} \times M$ with compact support.

(5-38) - Let $U \subset \mathbb{R} \times M$ denote a compact set. Then
(a) $\lim _{n \rightarrow \infty} \sup _{z \in \bigcup_{(C, m) \in \vartheta_{k^{\prime}}}(C \cap U)}$
$\operatorname{dist}(z$
(b) $\lim _{n \rightarrow \infty} \sup _{z \in \bigcup_{(C, m) \in \vartheta_{k, n}}\left(\phi_{k^{\prime}, n}(C) \cap U\right)}$
$(C, m) \in \vartheta_{k, n}$
$\left.\phi_{k^{\prime}, n}(C)\right)=0$
$\operatorname{dist}\left(z, \bigcup_{(C, m) \in \vartheta_{k^{\prime}}} C\right)=0$. 
This convergence follows using the arguments in [6, Section 6], or by using the results about convergence of $J$-holomorphic curves on symplectizations as can be found in Bourgeois et al [1]. Granted (5-38), replace each $n \in\{1,2, \ldots\}$ version of $\vartheta_{k, n}$ by the version of $\left\{\vartheta_{k^{\prime}}: k^{\prime} \in \Lambda_{k}\right\}$ that is obtained by translating the subvarieties from any given $\vartheta_{k^{\prime}}$ by the translation along the $\mathbb{R}$ factor of $\mathbb{R} \times M$ that is given by $\phi_{k^{\prime}, n}^{-1}$. It follows from (5-38) that this replacement for $\vartheta_{k, n}$ obeys all of Proposition 5.5's requirements.

\section{Proof of Theorem 1.2}

The proof begins by assuming the theorem is false so as to generate some nonsense. In particular, were it false, then there exists a sequence $\left\{\left(r_{n},\left(A_{n}, \psi_{n}\right)\right)\right\}_{n=1,2, \ldots}$ such that

- The sequence $\left\{r_{n}\right\}_{n=1,2, \ldots} \subset[1, \infty)$ is increasing and unbounded.

- Any given $\mathfrak{d}_{n}=\left(A_{n}, \psi_{n}\right)$ is a solution to the $r=r_{n}$ version of (1-11) in $\mathcal{M}_{1}\left(\mathfrak{c}_{-}, \mathfrak{c}_{+}\right)$.

- $\mathfrak{d}_{n}$ is not in the image of $\Psi^{r}$.

The remaining subsections generate some nonsense from this assumption by proving that these conditions are not mutually consistent.

\section{6.a Finding $\vartheta \in \mathcal{M}_{1}\left(\Theta_{-}, \Theta_{+}\right)$}

An element, $\vartheta$, in $\mathcal{M}_{1}\left(\Theta_{-}, \Theta_{+}\right)$can be viewed as a finite set whose typical element has the form $(C, m)$ with $C \subset \mathbb{R} \times M$ an embedded, pseudoholomorphic subvariety and with $m$ a positive integer which is equal to 1 if $C$ is not $\mathbb{R}$-invariant. The subvariety components of different pairs from $\vartheta$ are distinct and disjoint. In addition, there is precisely one such component that is not $\mathbb{R}$-invariant.

Lemma 6.1 There an element $\vartheta \in \mathcal{M}_{1}\left(\Theta_{-}, \Theta_{+}\right)$, a subsequence of $\left\{\left(A_{n}, \psi_{n}\right)\right\}_{n=1,2, \ldots}$ (hence renumbered consecutively from 1 ) and a corresponding sequence of constant translations along the $\mathbb{R}$ factor of $\mathbb{R} \times M$, all with the following property: For each $n$, write the translated version of $\psi_{n}$ as a pair $\left(\alpha_{n}, \beta_{n}\right)$. The sequence with $n$-th element

$$
\sup _{z \in \cup_{(C, m) \in \vartheta} C} \operatorname{dist}\left(z, \alpha_{n}^{-1}(0)\right)+\sup _{z \in \alpha_{n}^{-1}(0)} \operatorname{dist}\left(\bigcup_{(C, m) \in \vartheta} C, z\right)
$$

converges with limit zero. In addition, if $\mathbb{I} \subset \mathbb{R}$ is an interval of length 1 and $v$ is a 2-form on $\mathbb{R} \times M$ with $\|v\|_{\infty}=1$ and support on $I \times M$, then the sequence whose $n$-th element is $(i /(2 \pi)) \int_{\mathbb{R} \times M} v \wedge F_{\hat{A}}-\sum_{(C, m) \in \vartheta} m \int_{C} v$ also converges with limit zero. 


\section{Proof of Lemma 6.1 The proof has two parts.}

Part 1 Proposition 5.5 can be brought to bear with if $n$ is sufficiently large. By passing to a subsequence and renumbering the latter consecutively from 1, the proposition can be applied using $r_{n}$ and $\left(A_{n}, \psi_{n}\right)$ with $\delta=1 / n$. Each sufficiently large $n$ application of this proposition produces a collection $\left\{\vartheta_{k, n}\right\}_{k=1, \ldots, N_{n}}$ of some $N_{n}$ pairs as described in (b) of the second item of the proposition. Note in this regard that $N_{n}$ has an $n-$ independent upper bound. By virtue of what is said in (b) of the second item of the proposition, the subsequence can be winnowed further so that $N_{n}=N$ is independent of $n$, and so that the sequence $\left\{\left\{\vartheta_{k, n}\right\}_{k=1, \ldots, N}\right\}_{n=1,2, \ldots}$ has a subsequence that converges to a broken trajectory between $\Theta_{-}$and $\Theta_{+}$where the convergence is as described in the proof of Proposition 5.5 and (5-38). The manner of convergence is of the sort that is described in the proof of Proposition 5.1 and (5-38). This broken trajectory consists of an ordered collection $\left\{\vartheta_{k}\right\}_{k=1,2, \ldots, N^{\prime}}$ as described in the proof of Proposition 5.5: First, the typical element in each set is a pair $(C, m)$ where $C \subset \mathbb{R} \times M$ is a pseudoholomorphic subvariety and where $m$ is a positive integer. As usual, no two pairs from any given $\vartheta_{k}$ share the same subvariety. Moreover, at least one pair from $\vartheta_{k}$ has a subvariety that is not $\mathbb{R}$-invariant. There are also the constraints on the $s \rightarrow \pm \infty$ limits of the pairs from $\vartheta_{k}$, using the notation from the proof of Proposition 5.5, these are

$$
\Theta_{1-}=\Theta_{-}, \quad \Theta_{k-}=\Theta_{k-1,+} \text { for } k>1 \text { and } \Theta_{N+}=\Theta_{+} .
$$

Part 2 Suppose that $c_{1}(\operatorname{det}(\mathbb{S}))$ is torsion: Given that the embedded contact homology index $I\left(\Theta_{-}, \Theta_{+}\right)=1$ and given that $J_{a}$ comes from $g_{a}$, what is said in Hutchings [2, Lemma 9.5] (see also Hutchings and Sullivan [4, Corollary 11.5] and Hutchings [3, Theorem 5.1]) prove that $N^{\prime}=1$ and that $\vartheta=\vartheta_{1} \in \mathcal{M}_{1}\left(\Theta_{-}, \Theta_{+}\right)$. To elaborate, any given $\vartheta_{k} \in\left\{\theta_{k^{\prime}}\right\}_{1 \leq k^{\prime} \leq N^{\prime}}$ defines a relative class in $H_{2}\left(M ; \Theta_{k-}, \Theta_{k+}\right)$ as described in [8, Section 2.c]. Let $\left[Z_{k}\right]$ denote this class. Hutchings uses $\left[Z_{k}\right]$ to define via [8, (2-9)] the integer $I\left(\Theta_{k-}, \Theta_{k+}, Z_{k}\right)$. This is the embedded contact homology index of $\vartheta_{k}$. By virtue of (6-2), $\sum_{1 \leq k \leq N^{\prime}} I\left(\Theta_{k^{\prime}}, \Theta_{k^{\prime}}, Z_{k}\right)$ is equal to 1 . If $N^{\prime}>1$, then there must exist at least on element $\left\{\vartheta_{k}\right\}_{1 \leq k \leq N^{\prime}}$ whose embedded-contact homology index is nonpositive. As such element must contain a pair with a non- $\mathbb{R}$-invariant subvariety, [2, Lemma 9.5] proves that this is not possible given that $J$ is in the set $g_{a}$. Given that $N^{\prime}=1$ and given that the one element is in $\mathcal{M}_{1}\left(\Theta_{-}, \Theta_{+}\right)$, the conclusions of the lemma follow from Proposition 5.5 using what is said in (5-38) about convergence.

Suppose that $c_{1}(\operatorname{det}(\mathbb{S}))$ is not torsion. Each $k \in\left\{1, \ldots, N^{\prime}\right\}$ has its associated embedded contact homology index $I\left(\Theta_{k-}, \Theta_{k+}, Z_{k}\right)$ as in the previous case. Given (6-2) the collection $\left\{Z_{k}\right\}_{1 \leq k \leq N^{\prime}}$ defines a class $[Z] \in H_{2}\left(M, \Theta_{-}, \Theta_{+}\right)$and thus the 
integer $I\left(\Theta_{-}, \Theta_{+}, Z\right)$. As argued momentarily, this integer is equal to 1 . Given that $I\left(\Theta_{-}, \Theta_{+}, Z\right)=1$, the arguments from the previous case can be applied to prove that $N=1$ and that $\vartheta=\vartheta_{1} \in \mathcal{M}_{1}\left(\Theta_{-}, \Theta_{+}\right)$. What follows explains why $I\left(\Theta_{-}, \Theta_{+}, Z\right)=1$.

To start the explanation, fix a surface $\Sigma \subset \mathbb{R} \times M$ as described in [10, Part 1 of Section 2.b] whose projection to $M$ defines the class [Z]. Let $\mathfrak{c}_{-}=\left(A_{-}, \psi_{-}=\left(\alpha_{-}, \beta_{-}\right)\right)$ and $\mathfrak{c}_{+}=\left(A_{+}, \psi_{+}=\left(\alpha_{+}, \beta_{+}\right)\right)$denote the respective $s \rightarrow-\infty$ and $s \rightarrow \infty$ limits of a given large $n$ version of $\left(A_{n}, \psi_{n}\right)$. The fact that $\Sigma$ defines the class $Z$ implies (via Alexander duality) that the normal bundle of $\Sigma$ extends over $\mathbb{R} \times M$ as the bundle $E$ and, given a tubular neighborhood of $\Sigma$, there is a pair, $(A, \alpha)$, of connection on $E$ and section of $E$ with the following properties: First, $\alpha$ vanishes transversally and its zero locus is $\Sigma$. Second, $\alpha$ is $A$-covariantly constant on the complement of the given tubular neighborhood of $\Sigma$.

Use $\Sigma$ as in [10, Section 2.c] to define the integer $k_{\Sigma}$ and the corresponding operator $\mathfrak{D}_{E}$. Granted what was said in the preceding paragraph, [10, Lemma 2.5] finds that the index of the $\mathfrak{d}=\left(A_{n}, \psi_{n}\right)$ version of (1-12) is equal to index $\left(\mathfrak{D}_{E}\right)+2 k_{\Sigma}$, and thus the latter is equal to 1 . Meanwhile, [10, Lemmas 2.7 and 2.8] with [10, (2-109)] assert that the index of $\mathfrak{D}_{E}$ is equal to $I\left(\Theta_{-}, \Theta_{+}, Z\right)-2 k_{\Sigma}$. These last two conclusions imply the desired result: $I\left(\Theta_{-}, \Theta_{+}, Z\right)=1$.

Lemma 6.1 has the refinement that follows.

Lemma 6.2 The sequence of translations for Lemma 6.2 can be chosen so that the following is also true: Fix $\delta_{*}>0$ and then there exists $n_{*}$ such that if $n>n_{*}$,

$$
\sup _{z \in \bigcup_{(C, m) \in \vartheta}} \operatorname{dist}\left(z, \alpha_{n}^{-1}(0)\right)+\sup _{z \in \alpha_{n}^{-1}(0)} \operatorname{dist}\left(\bigcup_{(C, m) \in \vartheta} C, z\right)<\delta_{*} r_{n}^{-1 / 2} .
$$

The proof of Lemma 6.2 is deferred to Section 7. Accept it for now.

\section{6.b The distance between $\left(A_{n}, \psi_{n}\right)$ and $\left(A^{*}, \psi^{*}\right)$}

For each $n$, let $\mathfrak{d}_{n}=\left(A_{n}, \psi_{n}=\left(\alpha_{n}, \beta_{n}\right)\right)$ now denote the translate along the $\mathbb{R}$ factor of $\mathbb{R} \times M$ of the original $n$-th sequence element using the $n$-th translation from the sequence of translations that is described by Lemma 6.1 and Lemma 6.2. With $n$ specified, use $\left(A^{*}, \psi^{*}\right)$ in what follows to denote the $r=r_{n}$ version of the pair that is constructed in [9, Section 5.a]. 
Lemma 6.3 There exists $\kappa>0$ and, given $\delta>0$, there exists $n_{\delta} \geq 1$; and these are such that the following is true: If $n \geq n_{\delta}$, then there exists a smooth map $u=u_{n}: \mathbb{R} \times M \rightarrow S^{1}$ such that $\left(A_{n}-u^{-1} d u, u \psi_{n}\right)=\left(A^{*}+(2 r)^{1 / 2} b^{\prime}, \psi^{*}+\eta^{\prime}\right)$ with $\left|b^{\prime}\right|+\left|\eta^{\prime}\right| \leq \delta$ and $\left|\nabla b^{\prime}\right| \leq \kappa r^{1 / 2}$.

The remainder of this subsection contains the following.

Proof of Lemma 6.3 The following simplified notation is used: When $n$ is given, then $(A, \psi)$ is used in what follows to denote any given version of $\left(A_{n}, \psi_{n}\right)$. Also, $\left\{U_{C}\right\}_{(C, m) \in \vartheta},\left\{U_{\gamma-}\right\}_{\gamma \in \Xi_{\Sigma-}},\left\{U_{\gamma+}\right\}_{\gamma \in \Xi_{\Sigma+}}$ and $U_{0}$ are used to denote the $r=r_{n}$ versions of the sets that are described in $[9,(5-3)]$.

The argument that follows has five parts.

Part 1 Fix $n$ and trivialize $E$ over $U_{0}$ as $U_{0} \times \mathbb{C}$ as done in Step 2 of Part 1 of [9, Section 6.a]. It is a consequence of Proposition 5.1, Lemma 3.8, the first bullet of Lemmas 3.7, 3.8 and 6.2 that $|\alpha| \geq 1-c_{0} r^{-1}$ on $U_{0}$ if $n \geq c_{0}$. Granted that such is the case, there is a gauge transformation $u_{0}: U_{0} \rightarrow S^{1}$ such that $u_{0} \alpha=|\alpha| 1_{\mathbb{C}}$ where $1_{\mathbb{C}}$ is the trivializing section of $E$. Thus,

$$
\left|u_{0} \alpha-1_{\mathbb{C}}\right| \leq c_{0} r^{-1} .
$$

Let $A_{I}$ denote the connection on $E \rightarrow U_{0}$ that makes $1_{\mathbb{C}}$ covariantly constant and write $A=A_{I}+(2 r)^{1 / 2} b_{0}$. Given that Lemma 3.8 finds $\left|\nabla_{A} \alpha\right| \leq c_{0} r^{-1 / 2}$ and Lemma 3.6 finds $\left|\nabla_{A}^{2} \alpha\right| \leq c_{0} r$, it follows that

$$
\left|b_{0}\right|<c_{0} r^{-1} \text { and } \quad\left|\nabla b_{0}\right| \leq c_{0} r^{1 / 2} .
$$

Part 2 Given $(C, m) \in \vartheta$, use $\pi: N \rightarrow C$ to denote the normal bundle to $C$, and let $N_{1} \subset N$ denote the disk bundle that is described in [9, Section 4.a]. Reintroduce $e_{C}: N_{1} \rightarrow \mathbb{R} \times M$, this the exponential map from [9, Section 4.a].

Fix $(C, m) \in \vartheta$ and a point $p \in C$. Then fix complex Gaussian coordinates centered at $p$ of the sort that is described in Part 2 of Section 4.a, but chosen so that the coordinates, $x=\left(x_{0}, x_{1}\right)$, are such that the plane $x_{1}=0$ is tangent to $C$ at $p$ and the plane $x_{0}=0$ is tangent to $e_{C}\left(\left.N_{0}\right|_{p}\right)$. Let $\rho_{*}>0$ be such that the coordinates are defined for $|x| \leq \rho_{*}$. Rescale by the map $x \rightarrow r^{-1 / 2} x$ and let $\left(A_{n p}, \psi_{n p}=\left(\alpha_{n p}, \beta_{n p}\right)\right)$ denote the resulting pair of connection and spinor defined now on the radius $r^{1 / 2} \rho_{*}$ ball in $\mathbb{C}^{2}$. The zeros of $\alpha_{n p}$ have distance $\delta$ or less from the $x_{1}=0$ plane. Let $\left(A_{0}, \alpha_{0}\right)$ denote what is given for large $n$ by Lemma 4.3. Since $\alpha_{0}^{-1}(0)$ is a complex algebraic subvariety of $\mathbb{C}^{2}$ and since its zeros all have distance $\delta$ or less from the 
$x_{1}=0$ plane, so $\alpha_{0}^{-1}(0)$ must be a disjoint union of some $m$ or less planes, all of the form $x_{1}=$ constant. This implies that $\left(A_{0}, \alpha_{0}\right)$ is the pullback via the projection $x \rightarrow x_{1}$ from $\mathbb{C}^{2}$ to $\mathbb{C}$ of a vortex in $\mathfrak{C}_{m}$.

Granted the preceding, Lemma 6.2 and Lemma 4.3 have the following consequence: Given a positive integer $k$ and real numbers $\varepsilon>0$ and $T \geq 1$, the restriction of all sufficiently large $n$ versions of $\left(A_{n p}, \alpha_{n p}\right)$ to the radius $T$ ball in $\mathbb{C}^{2}$ has $C^{k}$ distance $\varepsilon$ or less to some gauge transformation of the pullback via $x \rightarrow x_{1}$ of the symmetric vortex in $\mathfrak{C}_{m}$.

Given $k, \varepsilon$ and $T$, the preceding conclusions do not yet hold for all points in $\bigcup_{(C, m) \in \vartheta} C$ with a bound on $n$ that is independent of the chosen point. The next lemma makes such a statement. To set the stage, suppose that a positive integer $n$ and a real number $\kappa>1$ have been specified. Given $(C, m) \in \vartheta$ and a point $p \in C$, let $\Lambda$ denote the set of triples that have the form $\left(q,\left(C^{\prime}, m^{\prime}\right)\right)$ such that $\left(C^{\prime}, m^{\prime}\right) \in \vartheta$ and $q \in C^{\prime} \cap e_{C}\left(\left.N_{1}\right|_{p}\right)$ has distance $\kappa r_{n}^{-1 / 2}$ or less from $p$. Set $m_{n \kappa}(p)=\sum_{\left(q,\left(C^{\prime}, m^{\prime}\right) \in \Lambda\right)} m^{\prime}$.

Lemma 6.4 Given a positive integer $k$ and real numbers $\varepsilon>0$ and $R \geq 1$, there exists $\kappa \geq 1$ with the following significance: Suppose that $n \geq \kappa$ and that $p \in \bigcup_{(C, m) \in \vartheta} C$. Define the pair $\left(A_{n p}, \alpha_{n p}\right)$ as in described above. This pair has $C^{k}$ distance $\varepsilon$ on the ball of radius $R$ in $\mathbb{C}^{2}$ to a gauge transformation of the pull back of a vortex in $\mathfrak{C}_{m_{n \kappa}}(p)$ via the projection $x \rightarrow\left(x_{0}, x_{1}\right)$ of $\mathbb{C}^{2}$ to $\mathbb{C}$.

Proof of Lemma 6.4 Remark first that there is an upper bound to the size of $m_{n c}(p)$ that is independent of $c$ and $p$. Let $m_{*}$ denote the latter. Now, let $(A, \alpha)$ denote a pair of connection on the trivial bundle over the radius $T$ ball in $\mathbb{C}^{2}$ and section over this ball. Suppose that $m^{\prime} \leq m_{*}$ and that $(A, \alpha)$ has $C^{k}$ distance less than $\frac{1}{2} \varepsilon$ from the pullback of a vortex, $\left(A_{0}, \alpha_{0}\right) \in C_{m^{\prime}}$ via the projection $x \rightarrow x_{1}$ from $\mathbb{C}^{2}$ to $\mathbb{C}$. Suppose, in addition that $m \leq m^{\prime}$ and that $m^{\prime}-m$ zeros of $\alpha_{0}$ have distance $T^{\prime} \geq 1$ or more from the origin in $\mathbb{C}^{2}$. Use what is said [9, Part 4 in Section 2.a] to draw the following conclusion: Given $k, \varepsilon$ and $T$, there exists $T_{*}$ such that if $T^{\prime}>T_{*}$, then any such $(A, \alpha)$ will have $C^{k}$ distance less than $\varepsilon$ on the ball of radius $T$ from the pullback of vortex in $\mathfrak{C}_{m}$.

Granted this, assume that the lemma is false so as to obtain a contradiction. If the lemma is false, there are $k, \varepsilon$ and $T$ and a sequence $\left\{p_{n}\right\}_{n=1,2, \ldots} \subset \bigcup_{(C, m) \in \vartheta} C$ with the following property: There are infinitely many integers $n \in\{1,2, \ldots\}$ such that the $p=p_{n}$ version of $\left(A_{n p}, \alpha_{n p}\right)$ has $C^{k}$ distance greater than $\varepsilon$ on the ball of radius $T$ in $\mathbb{C}^{2}$ from any gauge transformation of the pull back of any vortex from $\mathfrak{C}_{m_{n c}\left(p_{n}\right)}$ with $c=T_{*}$. Pass to a subsequence with this property and renumber consecutively from 1 . 
Even so, there exists $m \in\left\{1, \ldots, m_{*}\right\}$ and a subsequence of $\left\{\left(A_{n p}, \alpha_{n p}\right)\right\}_{n=1,2, \ldots}$ such that $m_{n c}\left(p_{n}\right)=m$, and such that a gauge transformation of each $\left(A_{n p}, \alpha_{n p}\right)$ makes the resulting sequence converge on compact subsets of $\mathbb{C}^{2}$ to a solution, $\left(A_{0}, \alpha_{0}\right)$, to (4-1). Moreover, there exists $z \geq 1$ such that the integral of $\left(1-\left|\alpha_{0}\right|^{2}\right)$ over any given $T^{\prime}>1$ ball centered on the origin in $\mathbb{C}^{2}$ is bounded by $z T^{\prime 2}$. As a consequence, $\alpha_{0}^{-1}(0)$ is a complex algebraic subvariety in $\mathbb{C}^{2}$. Each component of this subvariety has bounded distance from the plane $x_{1}=0$, and so the locus $\alpha_{0}^{-1}(0)$ is a disjoint union of planes on which $x_{1}$ is constant. Thus, $\left(A_{0}, \alpha_{0}\right)$ is the pullback of a vortex in some $\mathfrak{C}_{m^{\prime}}$ for $m^{\prime} \in\left\{m, \ldots, m_{*}\right\}$ via the projection $x \rightarrow x_{1}$ from $\mathbb{C}^{2}$ to $\mathbb{C}$.

With the preceding understood, each sufficiently large $n$ version of $\left(A_{n p}, \alpha_{n p}\right)$ will have $C^{k}$ distance $\frac{1}{2} \varepsilon$ or less on the ball of radius $T$ in $\mathbb{C}^{2}$ from some gauge transform of $\left(A_{0}, \alpha_{0}\right)$. What with the conclusions of the first paragraph of the proof, this implies that $\left(A_{n p}, \alpha_{n p}\right)$ has distance $\varepsilon$ or less on the ball of radius $T$ in $\mathbb{C}^{2}$ from a gauge transform of the pullback via the map $x \rightarrow x_{1}$ of a vortex in $\mathfrak{C}_{m}$. This violates the assumptions and so proves the lemma.

Part 3 Consider a given element $(C, m) \in \vartheta$ so as to discuss $(A, \alpha)$ on $U_{C}$. To this end, use Lemma 6.4 to conclude that there exists a map, $\hat{u}_{C}$, from the part of $U_{C}$ with distance $\frac{1}{4} \operatorname{Tr}^{-1 / 2}$ or less from $C$ to $S^{1}$ with certain desirable properties. To state the latter, write $\left(A-\widehat{u}_{C} d \widehat{u}_{C}^{-1}, \widehat{u}_{C} \psi\right)=\left(A^{*}+(2 r)^{1 / 2} b_{C 1}, \psi^{*}+\eta_{C 1}\right)$ where $\left(A^{*}, \psi^{*}\right)$ is described on $U_{C}$ in [9, Step 2 of Part 2 in Section 5.a]. According to what is said in Lemma 6.4, the gauge transformation $u_{C 1}$ can be chosen so that

$$
\left|b_{C 1}\right|+\left|\eta_{C 1}\right|+r^{-1 / 2}\left|\nabla b_{C 1}\right| \leq c_{0}\left(\varepsilon+R^{-1}\right)
$$

at points in $U_{C}$ with distance $\frac{1}{4} \operatorname{Tr}^{-1 / 2}$ or less from $C$ if $n \geq c_{0}$. To construct $\widehat{u}_{C}$, cover $C-\left(\bigcup_{\mathcal{E} \in_{E_{C}}} \mathcal{E}_{4 R}\right)$ by disks of radius $\operatorname{Tr}^{-1 / 2}$ so that the concentric disks of radius $\frac{1}{8} \operatorname{Tr}^{-1 / 2}$ are disjoint. This being the case, at most $c_{0}$ such disks can overlap. As a consequence, there is a partition of unity for this cover such that if $D$ is any given disk in the cover, then the norm of the derivative of the partition function with value 1 at $D$ 's center is bounded by $c_{0} T^{-1} r^{1 / 2}$ and the norm of its second derivative is bounded by $c_{0} T^{-2} r$. According to Lemma 6.4, there is a map, $u_{C D}$, from the ball of radius $\operatorname{Tr}^{-1 / 2}$ centered at the origin in $D$ to $S^{1}$ such that $\left(A-u_{C D}^{-1} d u_{C D}, u_{C D} \psi\right)$ can be written as a pair $\left(A^{*}+(2 r)^{1 / 2} b, \psi^{*}+\eta\right)$ where $b$ and $\eta$ obey (6-5) if $n \geq c_{0}$. As a consequence, the partition of unity can be used to patch these gauge transformations together where disks overlap to give the map $\widehat{u}_{C}$.

Note that (6-5) implies that $\widehat{u}_{C} \alpha$ differs from $\mathfrak{s}^{m} /|\mathfrak{s}|^{m}$ by $c_{0}\left(\varepsilon+T^{-1}\right)$ at points on where $|\mathfrak{s}| \in\left[\frac{1}{8} \operatorname{Tr}^{-1 / 2}, \frac{1}{4} \operatorname{Tr}^{-1 / 2}\right]$. According to what is said in Lemma 3.8, $|\alpha| \geq$ $1-c_{0} e^{-T / c_{0}}$ on the rest of $U_{C}$. This then allows for an extension of $\hat{u}_{C}$ from the 
$|\mathfrak{s}| \leq \frac{1}{8} \operatorname{Tr}^{-1}$ part of $U_{C}$ to the whole of $U_{C}$ so as to give a map $u_{C}: U_{\mathbb{C}} \rightarrow S^{1}$ with the following properties: First, $u_{C} \alpha=|\alpha| \mathfrak{s}^{m} /|\mathfrak{s}|^{m}$ at points where $|\mathfrak{s}| \geq \frac{1}{4} \operatorname{Tr}^{-1 / 2}$. To state the second, write $u_{C} \psi=\psi^{*}+\eta_{C}$ and write and $A-u_{C} d u_{C}{ }^{-1}$ as $A^{*}+(2 r)^{1 / 2} b_{C}$. Then

$$
\left|\eta_{C}\right|+\left|b_{C}\right| \leq c_{0}\left(\varepsilon+T^{-1}\right) \quad \text { and } \quad\left|\nabla b_{C}\right| \leq c_{0} r^{1 / 2}
$$

if $n \geq c_{0}$. Note in this regard that the bound on $\left|\nabla b_{C}\right|$ at points where $|\mathfrak{s}| \geq \frac{1}{8} \operatorname{Tr}^{-1 / 2}$ follows from the $q=2$ version of the third bullet of Lemma 3.6.

Given that $\mathfrak{s}^{m} /|\mathfrak{s}|^{m}$ is the transition function between the respective trivializations of $E$ over $U_{C}$ and over $U_{0}$, and given that $u_{C} \alpha=|\alpha| \mathfrak{s}^{m} /|\mathfrak{s}|^{m}$ and $u_{0} \alpha=|\alpha| 1_{\mathbb{C}}$ on $U_{C} \cap U_{0}$, it follows that the pair $\left(u_{0}, u_{C}\right)$ define a smooth map from $U_{0} \cup U_{C}$ to $S^{1}$.

Part 4 Fix $\gamma \in \Xi_{\Sigma-}$ so as to discuss $(A, \alpha)$ on $U_{\gamma-}$. Write $U_{\gamma-}$ as $(-\infty,-R] \times$ $S^{1} \times \mathbb{C}$ with coordinates $(w, t, z)$ as described in [9, (4-1)]. Fix $(C, m) \in \vartheta$ with $C \cap U_{\gamma-} \neq \varnothing$; and let $p$ denote a point in $C \cap U_{\gamma-}$. The exponential map $e_{C}$ gives a diffeomorphism between the restriction of $N_{1}$ to a neighborhood of $p$ and a neighborhood of $p$ in $U_{\gamma}$ that has certain special properties. To elaborate, write the coordinates $(w, t) \in(-\infty,-R] \times S^{1}$ of the point $p$ as $\left(w_{p}, t_{p}\right)$. A neighborhood of $p$ in $C$ has coordinates $(\widehat{w}, \hat{t})$ that are defined on a neighborhood of the origin in $\mathbb{R}^{2}$ and such that $C$ near $p$ is parametrized by $(\widehat{w}, \widehat{t}) \rightarrow\left(w=w_{p}+\widehat{w}, t=t_{p}+\widehat{t}, z=\right.$ $\left.z_{\mathcal{E}}\left(w_{p}+\hat{w}, t_{p}+\hat{t}\right)\right)$ with $z_{\mathcal{E}}(\cdot)$ given by the version of the map in (4-2) for the end $\mathcal{E} \subset C$ that contains $p$. As noted in Property 4 and (4-4) of Section 4.a, there is a trivialization of $C$ 's normal bundle on a neighborhood of $z$ so that with $\eta$ denoting the fiber coordinate for the normal bundle, then $e_{C}$ sends the coordinates $(\widehat{w}, \hat{t}, \eta)$ to the point

$$
\begin{aligned}
\left(w=w_{p}+\widehat{w}+\mathfrak{r}_{s}, t=t_{p}+\right. & \widehat{t}+\mathfrak{r}_{t}, \\
z & \left.=z_{\mathcal{E}}\left(w_{p}+\widehat{w}, t_{p}+\hat{t}\right)+\left(1+\mathfrak{r}_{z}\right)\left(\frac{\ell_{\gamma}}{2 \pi}\right)^{-1 / 2} \eta\right)
\end{aligned}
$$

in $U_{\gamma-}$. Here, $\mathfrak{r}_{s}, \mathfrak{r}_{t}$ and $\mathfrak{r}_{z}$ are as described in [9, (4-4)]. It then follows that the inverse diffeomorphism pulls back $\eta$ and $d \eta$ as

$$
\text { - }\left(e_{C}^{-1}\right)^{*} \eta=\left(\frac{\ell_{\gamma}}{2 \pi}\right)^{1 / 2}\left(z-z_{\mathcal{E}}\right)+\mathfrak{e}
$$

$$
\text { - }\left(e_{C}^{-1}\right)^{*} d \eta=\left(\frac{\ell_{\gamma}}{2 \pi}\right)^{1 / 2}\left(d z-\left(\frac{\partial}{\partial s} z_{\mathcal{E}}\right) d w-\left(\frac{\partial}{\partial t} z_{\mathcal{E}}\right) d t\right)+\mathfrak{e}^{\prime},
$$

where $|\mathfrak{e}| \leq c_{0}|z|^{2}|\eta|$ and $\left|\mathfrak{e}^{\prime}\right| \leq c_{0}\left(|\eta||z|+|z|^{2}\right)$. 
Granted (6-7) and (6-8), it then follows from Lemma 6.4 together with $[9,(2-4)]$ that there is a map, $\widehat{u}$, from the ball of radius $\frac{3}{4} \operatorname{Tr}_{\gamma}^{-1 / 2}$ in $U_{\gamma}$ - centered at $p$ to $S^{1}$ with the following property: Introduce $\left(A^{*}, \psi^{*}\right)$ from $[9,(5-8)]$. Then $\left(A-\hat{u}^{-1} d \hat{u}, \widehat{u} \psi\right)$ can be written in this ball as $\left(A^{*}+(2 r)^{1 / 2} b, \psi^{*}+\eta\right)$ where

$$
|\eta|+|b|+r^{1 / 2}|\nabla b| \leq c_{0}\left(\varepsilon+r^{-3 \sigma}\right)
$$

if $n \geq c_{0}$.

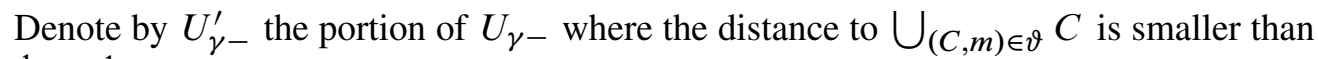
$\frac{1}{4} \operatorname{Tr}_{\gamma}^{-1}$. Granted (6-9), an argument like the one used in Part 3 invoking [9, (2-4)] gives a map $\hat{u}_{\gamma-}: U_{\gamma-}^{\prime} \rightarrow S^{1}$ such that $\left(A-\hat{u}_{\gamma-}^{-1} d \widehat{u}_{\gamma-}, \widehat{u}_{\gamma-\psi}\right)=\left(A^{*}+(2 r)^{1 / 2} b_{\gamma-1}\right.$, $\left.\psi^{*}+\eta_{\gamma-1}\right)$ where the pair $\left(b_{\gamma-}, \eta_{\gamma_{-}}\right)$also obeys (6-9) with the addition to the right hand side of $c_{0} T^{-1}$.

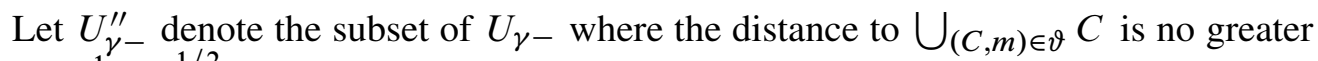
than $\frac{1}{8} \operatorname{Tr}_{\gamma}^{-1 / 2}$. Given [9, (2-4)] and given Lemma 3.8 and the final item of Lemma

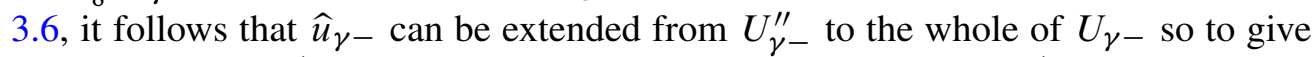
a map, $u_{\gamma-}$, to $S^{1}$ with the following properties: First, $\left(A-u_{\gamma}^{-1} d u_{\gamma-}, u_{\gamma-\psi}\right)=$ $\left(A^{*}+(2 r)^{1 / 2} b_{\gamma-}, \psi^{*}+\eta_{\gamma-}\right)$ where

$$
\left|\eta_{\gamma-}\right|+\left|b_{\gamma-}\right| \leq c_{0}\left(\varepsilon+T^{-1}\right) \quad \text { and } \quad\left|\nabla b_{\gamma-}\right| \leq c_{0} r^{1 / 2} \text {. }
$$

Second, the map $u_{\gamma-}$ and the map $u_{0}$ from Part 2 define a smooth map from $U_{0} \cup U_{\gamma-}$

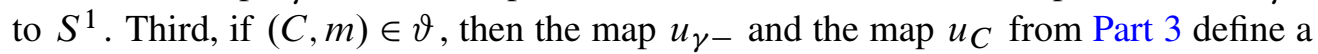
smooth map from $U_{C} \cup U_{\gamma-}$ to $S^{1}$. The construction of such an extension of $\hat{u}_{\gamma-}$ is straightforward and so the details are omitted except for the reminder to take into account the nontrivial transition functions on $U_{0} \cap U_{\gamma}$ and on $U_{\gamma} \cap \cap U_{C}$ that are used in [9, Section 5.a] for the description of $\left(A^{*}, \psi^{*}\right)$.

As might be imagined, there is a map $\hat{u}_{\gamma+}$ from any $\gamma \in \Xi_{\Sigma+}$ version of $U_{\gamma+}$ with the analogous properties.

Part 5 As noted, the maps $\left\{u_{0},\left\{u_{C}\right\}_{(C, m) \in \vartheta},\left\{u_{\gamma-}\right\}_{\gamma \in \Xi_{\Sigma-}},\left\{u_{\gamma+}\right\}_{\gamma \in \Xi_{\Sigma+}}\right\}$ patch together so as to define a single map smooth, $u: \mathbb{R} \times M \rightarrow S^{1}$. This being the case, write the pair $\left(A-u^{-1} d u, u \psi\right)$ as $\left(A^{*}+(2 r)^{1 / 2} b^{\prime}, \psi^{*}+\eta^{\prime}\right)$ and it follows from

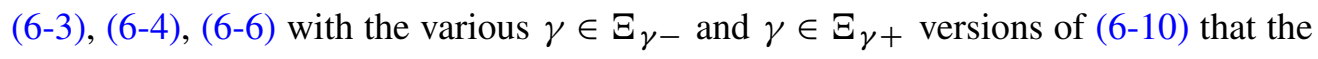
bound claimed by Lemma 6.3 holds if $n \geq c_{0}$.

\section{6.c Equation (5-20) of [9]}

Reintroduce the space $\mathcal{K}_{*}$ as defined at the end of [9, Step 4 in Section 5.a]. Section 5.a of [9] assigns to any given element $\xi \in \mathcal{K}_{*}$ a pair $\left(A^{\xi}, \psi^{\xi}\right)$ of connection on $E \rightarrow \mathbb{R} \times M$ 
and section over $\mathbb{R} \times M$ of $S$. A formula for this pair is given by [9, (5-15)]. Write this pair $\left(A^{\xi}, \psi^{\xi}\right)$ as $\left(A^{*}, \psi^{*}\right)+\mathfrak{t}^{\xi}$ where $\mathfrak{t}^{\xi}$ is viewed as a section over $\mathbb{R} \times M$ of $i T^{*}(\mathbb{R} \times M) \oplus \mathbb{S}$.

Fix some large $n$, and introduce from Lemma 6.3 the gauge transformation $u_{n}$ so as to write

$$
(A, \psi)=\left(A_{n}-u_{n}^{-1} d u_{n}, u_{n} \psi_{n}=\left(A^{*}+(2 r)^{1 / 2} b^{\prime}, \psi^{*}+\eta^{\prime}\right)\right.
$$

with $\left|b^{\prime}\right|+\left|\eta^{\prime}\right| \leq \delta$ and $\left|\nabla b^{\prime}\right| \leq c_{0} \kappa r^{1 / 2}$. With $\xi \in \mathcal{K}_{*}$ given, write

$$
(A, \psi)=\left(A^{\xi}, \psi^{\xi}\right)+\left((2 r)^{1 / 2}\left(\phi^{\xi} d s+b^{\xi}\right), \eta^{\xi}\right) .
$$

This is to say that $\left((2 r)^{1 / 2}\left(\phi^{\xi} d s+b^{\xi}\right), \eta^{\xi}\right)=\left((2 r)^{1 / 2} b^{\prime}, \eta^{\prime}\right)-\mathfrak{t}^{\xi}$. To make the connection with what is done in $\left[9\right.$, Sections 5.d and 6], note that $\left(b^{\xi}, \eta^{\xi}, \phi^{\xi}\right)$ obeys the top two equations in $[9,(5-20)]$. By way of reminder, the three equations in [9, (5-20)] assert that a section $\mathfrak{b}=(b, \eta, \phi)$ over $\mathbb{R} \times M$ of $i T^{*} M \oplus \mathbb{S} \oplus i \mathbb{R}$ obeys

$$
\begin{aligned}
& -\frac{\partial}{\partial s} b+* d_{M} b-d_{M} \phi-2^{-1 / 2} r^{1 / 2}\left(\psi^{\xi \dagger} \tau \eta+\eta^{\dagger} \tau \psi^{\xi}\right)-2^{-1 / 2} r^{1 / 2} \eta^{\dagger} \tau \eta \\
& =-2^{-1 / 2} r^{-1 / 2}\left(\frac{\partial}{\partial s} A^{\xi}+B_{A^{\xi}}-r\left(\psi^{\xi \dagger} \tau \psi^{\xi}-i a\right)-\left(i * d \mu+\frac{1}{2} B_{A_{K}}\right)\right) .
\end{aligned}
$$

$$
\begin{aligned}
- & \left(\nabla_{A^{\xi}}\right)_{S} \eta+D_{A^{\xi}} \eta+2^{1 / 2} r^{1 / 2}\left(\operatorname{cl}(b) \psi^{\xi}+\phi \psi^{\xi}\right)+2 r^{1 / 2}(\operatorname{cl}(b) \eta+\phi \eta) \\
& =-\nabla_{s} \psi^{\xi}-D_{A^{\xi}} \psi^{\xi} .
\end{aligned}
$$$$
\text { - } \frac{\partial}{\partial s} \phi+* d_{M} * b-2^{-1 / 2} r^{1 / 2}\left(\eta^{\dagger} \psi^{\xi}-\psi^{\xi \dagger} \eta\right)=0 \text {. }
$$

In this equation, $d_{M}$ refers to the exterior derivative along the $M$ factor in $\mathbb{R} \times M$. An additional gauge transformation must be done to $(A, \psi)$ so as to obtain a new version of $\left(b^{\xi}, \eta^{\xi}, \phi^{\xi}\right)$ that obeys all three equations in (6-11), and thus all of [9, Property 1 of Section 5.d].

Lemma 6.5 Given $\delta>0$, there exists $\kappa \geq 1$ with the following significance: Suppose that $n \geq \kappa$ and that $\xi \in \mathcal{K}_{*}$ with $\|\xi\|_{\infty} \leq \kappa^{-1}$. There exists a continuous, locally $L_{2}^{2}$ map $u=u_{n}: \mathbb{R} \times M \rightarrow S^{1}$ such that $\left(A_{n}-u^{-1} d u, u \psi_{n}\right)=\left(A^{\xi}+(2 r)^{1 / 2} b, \psi^{\xi}+\eta\right)$ where $\mathfrak{b}(\xi)=(b, \eta)$ is a locally $L_{1}^{2}$ section of $i T^{*}(\mathbb{R} \times M) \oplus \mathbb{S}$ whose components obeys (6-11)when viewed as a section of $i T^{*} M \oplus \mathbb{S} \oplus i \mathbb{R}$. Moreover, the spinor component, $\eta$, has norm less than $\delta$; and if $s \in \mathbb{R}$, then

$$
\int_{[s, s+1] \times M}|\mathfrak{b}(\xi)|^{2} \leq \kappa \delta .
$$

Finally, $u$ is a smooth map if $\xi$ is a smooth element in $\mathcal{K}_{*}$. 
A slightly stronger assumption is required so as to obtain a uniform bound on all components of $\mathfrak{b}^{\xi}$. This assumption is stated in the next lemma. This next lemma plays no role in the subsequent arguments.

Lemma 6.6 Suppose that $v_{*} \in(0,1)$ has been given. Then there is a constant, $\kappa_{*} \geq 1$, and, given $\delta$, a constant $\kappa$ for use in Lemma 6.5 such that the following is true: Let $\sigma$ denote the constant from [9, Section 4.c], and suppose that $\sigma<\kappa_{*}^{-1} v_{*}$. Assume in addition that $n$ and $\xi$ are as described in Lemma 6.5, and that $\xi$ is Holder continuous with exponent $v_{*}$ and corresponding Holder norm bounded by $\kappa_{*}^{-1} r^{1 / 2} \delta$. Then $\mathfrak{b}(\xi)$ from Lemma 6.5 obeys $|\mathfrak{b}| \leq \delta$. In particular, if $z \geq 1$, if $n$ is sufficiently large and if $\|\xi\|_{\mathcal{K}} \leq z$, then $|\mathfrak{b}(\xi)| \leq \delta$.

Proof of Lemma 6.5 and Lemma 6.6 Given that $(A, \psi)$ is an instanton, all three items of (6-11) hold if the gauge transformation is chosen so that $\mathfrak{b}=(b, \eta, \phi)$ obeys the third item of (6-11). Given what is said about $\left|b^{\prime}\right|+\left|\eta^{\prime}\right|$ by [9, Lemma 6.3 and Lemma 2.6], the desired bounds on $|\mathfrak{b}|$ are guaranteed if $n$ is large, and if $\|\xi\|_{\infty}$ and $|u-1|$ and $r^{-1 / 2}|d u|$ are all suitably small. The five steps that follow explain how to find such a gauge transformation.

Step 1 The approach is much like that used Part 6 of Section 2.a. To say more, write the map $u$ as $u=e^{s}$ with $s$ a map from $\mathbb{R} \times M$ to $i \mathbb{R}$. The desired triple $(b, \eta, \phi)$ is determined by $s$ and $\left(b^{\xi}, \eta^{\xi}, \phi^{\xi}\right)$ via

(6-12) $b=b^{\xi}-(2 r)^{-1 / 2} d_{M} s, \quad \phi=\phi^{\xi}-(2 r)^{-1 / 2} \frac{\partial}{\partial s} s, \quad \eta=e^{s} \psi^{\xi}-\psi^{\xi}+e^{s} \eta^{\xi}$.

Equation (6-11) is obeyed if $s$ obeys an equation having the schematic form

$$
d^{\dagger} d s+2 r\left|\psi^{*}\right|^{2} s+\mathfrak{R}(s)-\mathfrak{P}_{b}-\mathfrak{P}_{\eta}=0,
$$

where the notation is as follows: First $d^{\dagger}$ denotes the formal $L^{2}$ adjoint of the exterior derivative on $\mathbb{R} \times M$. Second,

$$
\begin{aligned}
\mathfrak{R}(s)=r\left(e^{-s}-e^{s}+2 s\right)\left|\psi^{\xi}\right|^{2}+ & r\left(e^{s}-e^{-s}\right)\left(\left|\psi^{\xi}\right|^{2}-\left|\psi^{*}\right|^{2}\right) \\
& -r\left(\left(e^{-s}-1\right) \eta^{\xi \dagger} \psi^{\xi}-\left(e^{s}-1\right) \psi^{\xi \dagger} \eta^{\xi}\right),
\end{aligned}
$$

$$
\text { - } \mathfrak{P}_{b}=(2 r)^{1 / 2}\left(-\frac{\partial}{\partial s} \phi^{\xi}+d_{M}^{\dagger} b^{\xi}\right) \quad \text { and } \quad \mathfrak{P}_{\eta}=r l\left(\eta^{\xi \dagger} \psi^{\xi}-\psi^{\xi \dagger} \eta^{\xi}\right) \text {. }
$$

Note in particular that $|\Re(s)| \leq c_{0} r|s|\left(\left|\eta \eta^{\xi}\right|+\|\xi\|_{\infty}+|s|\right)$.

With regards to the size of $\left(b^{\xi}, \eta^{\xi}, \phi^{\xi}\right)$, remark that

$$
\left|\left(b^{\xi}, \eta^{\xi}, \phi^{\xi}\right)\right| \leq c_{0}\left(\left|b^{\prime}\right|+\left|\eta^{\prime}\right|+\|\xi\|_{\infty}+r^{-1 / 2}\right) .
$$


As a consequence, if $\varepsilon>0$ is given apriori, then the norm of $\left(b^{\xi}, \eta^{\xi}, \phi^{\xi}\right)$ can assumed to be less than $\varepsilon$ if $\|\xi\|_{\infty} \leq c_{0}^{-1} \varepsilon$ and if $n$ is large. This understood, fix $\varepsilon>0$ and assume that $n$ and $\xi$ are such that $\left|\left(b^{\xi}, \eta^{\xi}, \phi^{\xi}\right)\right|<\varepsilon$.

A solution to (6-13) is found by writing the latter as the equation for the fixed point of a mapping from $C^{0}(\mathbb{R} \times M ; i \mathbb{R})$ to itself. The map has the form $s \rightarrow \mathrm{T}(s)$ where

$$
\left.\mathrm{T}(s)\right|_{x}=\int_{\mathbb{R} \times M} G(x, \cdot)\left(-\mathfrak{R}(s)+\mathfrak{P}_{\mathrm{b}}+\mathfrak{P}_{\mathrm{J}}\right),
$$

where $G(x, p)$ denotes the Green's function for the operator $d^{\dagger} d+2 r\left|\psi^{*}\right|^{2}$ on $\mathbb{R} \times M$ with pole at the point $p$. It follows from the second item of the upcoming Lemma 6.7 that a fixed point of $\mathrm{T}$ in $C^{0}(\mathbb{R} \times M ; i \mathbb{R})$ is, in fact, a smooth function when $\xi$ is smooth. In this case, the equation $\mathrm{T}(s)=s$ can be differentiated twice to see that (6-13) is obeyed. In the case, when $\xi$ is not smooth, a fixed point of (6-16) is none the less a locally $L_{2}^{2}$ function that obeys (6-13). This follows using standard properties of the Laplacian on $\mathbb{R} \times M$. Indeed, the fixed point $s$ is bounded and so it is a locally $L^{2}$, weak solution to the equation $d^{\dagger} d s=-r\left|\psi^{*}\right|^{2} s+\mathfrak{K}$, where $\mathfrak{K}=-\mathfrak{R}(s)+\mathfrak{P}_{b}+\mathfrak{P}_{\eta}$ is a locally $L^{2}$ function on $\mathbb{R} \times M$.

Step 2 This step constitutes a digression to consider integrals such as the one on the right hand side of (6-16). The following lemma says what is needed for now.

Lemma 6.7 There exists a constant $\kappa \geq 1$ with the following significance: Take $r \geq \kappa$ and let $G$ denote the Green's function for the operator $d^{\dagger} d+r$. Suppose that $f$ is a smooth, bounded section of $i T^{*}(\mathbb{R} \times M)$ and $m$ is a bounded, $i \mathbb{R}$ valued function. Let $x$ denote the $i \mathbb{R}$ valued function on $\mathbb{R} \times M$ that obeys

$$
\left.\chi\right|_{x}=\int_{\mathbb{R} \times M} G(x, \cdot)\left(r^{1 / 2} d^{\dagger} f+r m\right) .
$$

Then $|\chi| \leq \kappa\left(\|f\|_{\infty}+\|m\|_{\infty}\right)$.

The proof of this lemma requires the following analog of Lemma 2.8:

Lemma 6.8 There exists $\kappa>0$ such that

- $\int_{\mathbb{R} \times M} G(x, \cdot) \leq \kappa r^{-1}$.

- $\int_{\mathbb{R} \times M}|d G|(x, \cdot) \leq \kappa r^{-1 / 2}$.

- Fix $\rho>0$. Then

(a) $\int_{\operatorname{dist}(x, \cdot)>\rho}|d G \overleftarrow{d}|(x, \cdot) \leq \kappa\left(1+\left|\ln \left(\rho r^{1 / 2}\right)\right|\right)$,

(b) $\int_{\operatorname{dist}(x, \cdot)>\rho}|d G|(x, \cdot) \operatorname{dist}(x, \cdot)^{-1} \leq \kappa\left(1+\left|\ln \left(\rho r^{1 / 2}\right)\right|\right)$,

at each $x \in M$. 
Proof of Lemma 6.8 The proof is much like that of Lemma 2.8. To start, fix $\varepsilon>0$ and let $g(\cdot, p)$ denote the Green's function for $d^{\dagger} d+2 \varepsilon^{2} r$ with pole at $p \in \mathbb{R} \times M$. The latter is strictly positive and it obeys

$$
\text { - } g(x, p) \leq c_{0} \frac{1}{\operatorname{dist}(x, p)^{2}} e^{-\varepsilon \sqrt{r} \operatorname{dist}(x, p) / c_{0}} .
$$

- $|d g|(x, p) \leq c_{0} \frac{1}{\operatorname{dist}(x, p)^{3}}\left(1+\varepsilon r^{1 / 2} \operatorname{dist}(x, p)\right) e^{-\varepsilon \sqrt{r} \operatorname{dist}(x, p) / c_{0}}$.

- $|d g \overleftarrow{d}|(x, p) \leq c_{0} \frac{1}{\operatorname{dist}(x, p)^{4}}\left(1+\varepsilon^{2} r \operatorname{dist}(x, p)^{2}\right) e^{-\varepsilon \sqrt{r} \operatorname{dist}(x, p) / c_{0}}$

Use the Green's function $g$ to write

$$
G(x, p)=g(x, p)-\int_{\mathbb{R} \times M} G(x, \cdot) r\left(\left|\psi^{*}\right|^{2}-\varepsilon^{2}\right) g(\cdot, p) .
$$

The Green's function $G$ is also positive, this a consequence of the maximum principle. It is also the case that $G(x, p)=G(p, x)$.

Fix $T \gg 1$ and integrate $G(\cdot, p)$ over the region where $|s-s(p)| \leq T$. View the result as a function of $p \in \mathbb{R} \times M$ and write its supremum as $r^{-1} m$. Note that this function has constant limits as $|s(p)| \rightarrow \pm \infty$, so $m$ is well defined. Given that $G>0$, it follows from (6-18) using the first item in (6-17) that

$$
m \leq c_{0} \varepsilon^{-2}+m r \sup _{p \in \mathbb{R} \times M} \int_{\left|\psi^{\prime}\right| \leq \varepsilon} g(\cdot, p) .
$$

What with (6-17), this last equation implies that $m$ obeys (2-27). This understood, it follows that if $\varepsilon \leq c_{0}$, then $m \leq c_{0} \varepsilon^{-2}$ which is independent of $T$.

To obtain what is claimed by the second item in Lemma 6.8, again fix $T \gg 1$. Differentiate (6-18) with respect to $p$. Take the absolute value of both sides and integrate with respect to $x$. Use the middle inequality in (6-17), the first assertion of the lemma and the fact that $G(x, p)=G(p, x)$ to see that the $|s-s(p)| \leq T$ portion of the integral in the second assertion of the lemma is bounded by $c_{0} r^{-1 / 2}$. As this is independent of $T$, the result follows.

To obtain Part (a) of the third assertion, fix $T \gg 1$. Differentiate (6-18) with respect to both $x$ and $p$; then use the results of the lemma's second assertion with the second and third items of (6-17) to obtain a bound by $c_{0}\left|\ln \left(\rho r^{1 / 2}\right)\right|$ on the contribution to the integral in question from the $|s-s(p)| \leq T$ part of $\mathbb{R} \times M$. As this is also independent of $T$, the result then follows. To obtain Part (b), first differentiate (6-18) with respect to $x$. Then take the norm of both sides, divide by $\operatorname{dist}(x, p)$ and integrate with respect 
to $p$. An upper bound by $\kappa\left|\ln \left(\rho r^{1 / 2}\right)\right|$ for the right hand side of the resulting expression follows using the second item of the lemma with the first item in (6-17).

Proof of Lemma 6.7 The bound on $x$ follows by using the first and second items of Lemma 6.8 after an integration by parts to take the derivative off of $f$ and replace it with a derivative from the right on $G$.

Step 3 Given Lemma 6.7, minor notational modifications to the argument given in Step 3 of the proof of Lemma 2.7 proves the following: There exists $c_{0}>2$ with the following significance: Set $\left|\left(b^{\xi}, \eta^{\xi}, \phi^{\xi}\right)\right|=\varepsilon$. If $\varepsilon<c_{0}^{-1}$, then the map $\mathrm{T}$ obeys

$$
\text { - }|\mathrm{T}(s)| \leq c_{0}^{-1}|s|+c_{0} \varepsilon \quad \text { if }|s| \leq c_{0}^{-1} \text {. }
$$

$$
\text { - }\left|\mathrm{T}(s)-\mathrm{T}\left(s^{\prime}\right)\right| \leq c_{0}^{-1}\left|s-s^{\prime}\right| \text { if both }|s| \text { and }\left|s^{\prime}\right| \text { are less than } c_{0}^{-1} \text {. }
$$

This then implies that if $\varepsilon<c_{0}^{-1}$, then $\mathrm{T}$ has a unique fixed point on the ball of radius $c_{0}^{-1}$ in $C^{0}(\mathbb{R} \times M ; i \mathbb{R})$; and this fixed point has sup norm bounded by $\varepsilon$. Let $s$ denote this fixed point.

Step 4 Suppose now that $v_{*} \in(0,1)$ has been specified and that $\xi$ is Holder continuous with exponent $v_{*}$. A bound on $|d s|$ by can be obtained by copying almost verbatim the arguments used in Step 4 of the proof of Lemma 2.7. There are but two salient changes and one additional observation. Here is are the changes: First, any given appeal to Lemma 2.8 is replaced by the appeal to the corresponding part of Lemma 6.8. To state the second, recall that the proof of Lemma 2.7 used the fact that $L_{1}^{2}$ functions on the circle are Holder continuous with exponent $\frac{1}{2}$. Appeal to this fact is replaced by the assumption Holder continuity assumption on $\xi$.

What follows is the important new observation: What is written as $\mathfrak{t}_{\xi}$ comes very close to obeying the third equation in (6-11) in that the derivatives of $\xi$ that arise in the $\mathfrak{t}_{\xi}$ contribution to $\mathfrak{P}_{b}$ appear with a factor bounded by $c_{0} \rho_{*}$. This being the case, a close look at the analysis of the terms in the $\mathbb{R} \times M$ version of (2-30) finds that the $k$-th such term is bounded by $c_{0} r^{1 / 2} 2^{-k v_{*} / c_{0}} \rho_{*} r^{-v_{*}}\|\xi\|_{C^{0, v}}$. The sequence of such bounds is again summable, and its sum is bounded by $r^{1 / 2} c_{0} v_{*}^{-1} \rho_{*} r^{-v}\|\xi\|_{C} 0, v$. The claim made by the lemma follows from this bound.

Step 5 Given the constant $\delta$ in Lemma 6.5, it follows from Steps 2-3 that $s$ exists. What is said in these steps also imply the following: If the expression on the right hand side of (6-15) is bounded by $c_{0}^{-1} \delta^{-4}$, then $|s|$ is bounded by $\delta^{2}$ if $\delta \leq c_{0}^{-1}$. It then follows that the spinor component of $\mathfrak{b}^{\xi}$ is bounded by $\delta$ if $\delta \leq c_{0}^{-1}$ and $n$ is large. 
With $b$ as written in (6-12), a bound on its $L^{2}$ norm over $[s, s+1] \times M$ follows from a bound on the $L^{2}$ norm of $r^{-1 / 2} d s$ over this same cylinder. To obtain such a bound, set $m$ to denote the function on $\chi(|s(\cdot)-s|-1)$ on $\mathbb{R} \times M$. Multiply both sides of (6-13) by $r^{-1} m s$, and then integrate the result over $\mathbb{R} \times M$. Integrate by parts so as to equate the integral of $r^{-1} m s d^{\dagger} d s$ with the integral of the function $-r^{-1} m|d s|^{2}-$ $r^{-1} \frac{1}{2}\left(d^{\dagger} d m\right)|s|^{2}$. The resulting equation equates the integral of $r^{-1} m|d s|^{2}$ with an integral over $[s-1, s+2] \times M$ whose integrand is bounded by $c_{0} \delta$. Since $m$ is a nonnegative function and equal to 1 on $[s, s+1] \times M$, this last equality leads directly to the $L^{2}$ bound asserted by Lemma 6.5.

The assertions made by Lemma 6.6 follow directly from what is said in Step 4 .

\section{6.d Choosing $\xi$}

To summarize what has been done so far, suppose that $\delta>0$ has been chosen. As demonstrated in the preceding, there exists $\kappa>1$ such that if $\xi \in \mathcal{K}_{*}$ with $\|\xi\|_{\infty}<\kappa^{-1}$, then the following is true: Define $\mathfrak{h}(\xi)$ as instructed in [9, Section 6.d]; and then introduce $\mathfrak{q}=\mathfrak{b}(\xi)-\mathfrak{h}(\xi)$. This section $q$ obeys

- $\mathfrak{D} \mathfrak{q}=r^{-1 / 2}\left(\mathfrak{v}-\mathfrak{v}_{\mathfrak{h}}\right)-r^{1 / 2} \mathfrak{q} * \mathfrak{q}-2 r^{1 / 2} \mathfrak{h} * \mathfrak{q}$.

- The $L^{2}$ norm of $\mathfrak{q}$ on any given cylinder of the form $[s, s+1] \times M$ is bounded by $\delta$.

- The spinor component of $q$ is bounded by $\delta$.

Here, the notation is that used in [9, Section 6]. The bounds on the $L^{2}$ norm of $\mathfrak{q}$ and on its spinor component follow from Lemma 6.5 and what is said about $\mathfrak{h}(\xi)$ in $[9$, Section 6.d].

Reintroduce from [9, Part 6 in Section 7.a] the projection $\Pi_{\xi}$ in order to defined the subspace $\mathbb{H}_{\xi} \frac{\perp}{\xi}=\left(1-\Pi_{\xi}\right) \mathbb{H}$. Likewise introduce the homomorphism $t_{\xi}: \mathcal{L} \rightarrow \mathbb{L}$ as defined in $[9,(6-9)]$ and its adjoint, $t_{\xi}^{\dagger}$. Since $\mathbb{H}_{\xi}^{\perp}$ is the kernel in $\mathbb{H}$ of $t_{\xi}^{\dagger}$, any element $\mathfrak{f} \in \mathbb{H}$ can be written uniquely as $\mathfrak{f}^{\perp^{\xi}}+t_{\xi}(\theta)$. Of interest here is the decomposition of $\mathfrak{q}$ in this manner as $\mathfrak{q}^{\perp}+t_{\xi}(\theta)$.

Lemma 6.9 There exists $\kappa>1$ with the following significance: Suppose that $\delta$ is positive but less than $\kappa^{-1}$ and that $n \geq \kappa$. Then there exists $\xi \in \mathcal{K}_{*}$ with $\|\xi\|_{\infty} \leq \delta$ such that $\Pi_{\xi}(\mathfrak{b}(\xi)-\mathfrak{h}(\xi))=0$. 
The rest of this subsection is occupied with the following.

Proof of Lemma 6.9 The proof has nine steps.

Step 1 This part says something about the size of $t_{\xi}^{\dagger}(\cdot)$ for any given $\xi \in \mathcal{K}_{*}$. To this end, fix $(C, m) \in \vartheta$ and a point $p \in C-\left(\bigcup_{\mathcal{E} \in \mathcal{E}_{C}} \mathcal{E}_{2 R}\right)$ and suppose that $\mathfrak{f}$ is a section of $i T^{*}(\mathbb{R} \times M) \oplus \mathbb{S}$ that is defined near $p$. It then follows using [9, (6-57)] that

$$
\left|\left(t_{\xi}^{\dagger}(\mathfrak{f})\right)_{C}\right| \leq c_{0}\left(1+\|\xi\|_{\infty}\right) \int_{\left.N\right|_{p}} \chi_{C}|\mathfrak{f}| r^{1 / 2} e^{-\sqrt{r}|\mathfrak{s}| / c_{0}}
$$

Here, $\pi: N \rightarrow C$ denotes $C$ 's normal bundle and $\mathfrak{s}$ denotes the canonical section over $N$ of $\pi^{*} N$. Similar integrals bound the other components of $\mathfrak{t}_{\xi}^{\dagger}(\mathfrak{f})$.

Step 2 Suppose that $\xi \in \mathcal{K}_{*}$ is any given element. It then follows from the definition given in [9, Section 6.d] of $\mathfrak{h}(\xi)$ and from [9, Lemma 3.10] that $t_{\xi}^{\dagger}(\mathfrak{h}(\xi))$ has $\|\cdot\|_{\infty}$ norm bounded by $c_{0}\left(\|\xi\|_{\infty}+1\right) r^{-3 / 4}$. Indeed, such is the case because any given $\mathfrak{b}_{C}$ from $[9,(6-54)]$ has vanishing $\mathbb{V}_{C 0}$ component, and because any given $\mathfrak{b}_{\gamma \pm}$ from [9, (6-43)-(6-44)] has vanishing $\mathbb{V}_{0}$ component.

To analyze the $t_{\xi}^{\dagger}$ image of $\mathfrak{b}(\xi)$, write the pair $\left(A^{\xi}, \psi^{\xi}\right)$ as $\left(A^{*}, \psi^{*}\right)+\left((2 r)^{1 / 2} c_{\xi}, \varsigma_{\xi}\right)$ with $\left(c_{\xi}, \varsigma_{\xi}\right)$ here viewed as a section of $i T^{*}(\mathbb{R} \times M) \oplus \mathbb{S}$. Likewise, view the $i T^{*} M$ and $i \mathbb{R}$ components of $\mathfrak{b}(\xi)$ as giving a section of $i T^{*}(\mathbb{R} \times M)$ and use the pair $\left(b^{\prime}, \eta^{\prime}\right)$ of Lemma 6.3 and the $i \mathbb{R}$ valued function $s=s(\xi)$ from the proof of Lemma 6.5 to write $\mathfrak{b}(\xi)=(b, \eta)$ with

$$
b=b^{\prime}-c_{\xi}-r^{-1 / 2} d s \text { and } \eta=e^{s} \eta^{\prime}-e^{s} \varsigma \xi+\left(e^{s}-1\right) \psi^{*} .
$$

Given $\delta>0$, Lemma 6.3 asserts that $\left|b^{\prime}\right|+\left|\eta^{\prime}\right| \leq \delta$ when $n$ is large. Meanwhile, $\left|c_{\xi}\right|+\left|\varsigma_{\xi}\right| \leq c_{0}\|\xi\|_{\infty}$.

Step 3 Equation (6-8) finds that $|s| \leq c_{0}\left(\delta+\|\xi\|_{\infty}\right)$. Lemma 6.6 can be used to bound $|d s|$ given a suitable bound for $\|\xi\|_{\mathcal{K}}$. However less is needed in what follows; only a bound for the $\|\cdot\|_{\xi}$ norm of $t_{\xi}^{\dagger}\left(\left(-r^{-1 / 2} d s,\left(e^{s}-1\right) \psi^{*}\right)\right)$ when $\xi \in \mathcal{K}_{*}$. The following lemma is used to obtain what is needed. The lemma augments the assertions made by Lemma 6.7.

Lemma 6.10 The constant $\kappa$ that appears in Lemma 6.7 can be chosen so that the following additional conclusion holds: Let $\xi \in \mathcal{K}_{*}$. Set $f=\left(-r^{-1 / 2} d \chi, \chi \psi^{*}\right)$ and then

- $\left\|t_{\xi}^{\dagger}(\mathfrak{f})\right\|_{\infty} \leq \kappa r^{-1 / 2}\left(r^{-1 / 2+6 \sigma}+\|\xi\|_{\infty}\right)\left(\|f\|_{\infty}+\|m\|_{\infty}\right)$.

- If, in addition, $\xi^{\prime} \in \mathcal{K}_{*}$, then

$$
\left\|t_{\xi}^{\dagger}(\mathfrak{f})-t_{\xi^{\prime}}^{\dagger}(\mathfrak{f})\right\|_{\infty} \leq c_{0} r^{-1 / 2}\left\|\xi-\xi^{\prime}\right\|_{\infty}\left(\|f\|_{\infty}+\|m\|_{\infty}\right) .
$$


Proof of Lemma 6.10 A preliminary digression is needed for some relevant background. To start this digression, return to the context in [9, Section 2] of the vortex equation on $\mathbb{C}$. For the purposes at hand, let $x$ denote a bounded function on $\mathbb{C}$. Let $\mathfrak{c}=(A, \alpha)$ denote a solution to (1-4), and introduce the corresponding version, $\vartheta_{\mathfrak{c}}$, of the operator depicted in (2-8). Then $\lambda=\left(-2^{1 / 2} \bar{\partial} \chi, \alpha \chi\right)$ can be written as $\vartheta_{\mathfrak{c}}^{\dagger}\left(2^{1 / 2} \chi, 0\right)$. As a consequence, $\lambda$ is $L^{2}$ orthogonal to the kernel of $\vartheta_{\mathfrak{c}}$. Note that the integral of the inner product between $\lambda$ and an element in the kernel of $\vartheta_{\mathfrak{c}}$ converges uniformly by virtue of $[9,(2-2)]$ and the fact that $|\chi|$ is bounded.

To explore the consequences of this last observation, consider first $(C, m) \in \vartheta$ and a point $p \in C-\left(\bigcup_{\mathcal{E} \in \mathcal{E}_{C}} \mathcal{E}_{2 R}\right)$. Suppose that $C$ is either not $\mathbb{R}$-invariant, or that its associated integer is 1 . Then $C$ 's component of $t_{\xi}^{\dagger}(\mathfrak{f})$ at $p$ can be written as

$$
\left(t_{\xi}^{\dagger}(\mathfrak{f})\right)_{C}=\int_{\left.N\right|_{p}} \chi_{C} \theta_{1 \xi=0 \cdot r}^{\dagger} \mathfrak{k}_{\xi}
$$

where the notation is as follows: First, $\theta_{1 \xi, r}$ is as defined in [9, (6-57)] and $\left.N\right|_{p}$ is the fiber at $p$ of $C$ 's normal bundle. Second, $\mathfrak{k}_{\xi}$ has components $\left(r^{-1 / 2} q_{\xi 0}(d \chi), q_{\xi 1}(\chi)\right)$ where $q_{\xi 0}(d \chi)$ is a linear combination of the $\mathbb{R} \times M$ components of $d \chi$ whose coefficients are bounded by $\left(c_{0}|\mathfrak{s}|+\|\xi\|_{\infty}\right)$ and have derivatives with norm bounded by $c_{0}\left(1+r^{1 / 2}\|\xi\|_{\infty}\right)$. Meanwhile, $\left|q_{\xi 1}(\chi)\right| \leq c_{0}\left(|s|+\|\xi\|_{\infty}\right)|\chi|$. Finally, the coefficients of $\left(q_{\xi 0}-q_{\xi^{\prime}}\right)(d \chi)$ and $q_{\xi 1}(x)-q_{\xi^{\prime} 1}(\chi)$ enjoy similar bounds but for the replacement of $\|\xi\|_{\infty}$ with $\left\|\xi-\xi^{\prime}\right\|_{\infty}$.

Consider now the proof of the first assertion. Given the bound on $|\chi|$ already noted, it follows using [9, (2-2)] that the contribution to (6-24) from the $q_{\xi 1}(\chi)$ part of $\mathfrak{k}_{\xi}$ is bounded by $c_{0} r^{-1 / 2}\left(r^{-1 / 2}+\|\xi\|_{\infty}\right)\left(\|f\|_{\infty}+\|m\|_{\infty}\right)$.

To consider the contribution to the integral in (6-24) from $q_{\xi 0}(d x)$, it proves useful to write $G(x, \cdot)$ as in (6-18) for the case $\varepsilon=1$. This done, define $\chi_{g}$ by the rule

$$
\left.\chi_{g}\right|_{x}=\int_{\mathbb{R} \times M} g(x, \cdot)\left(r^{1 / 2} d^{\dagger} f+r m\right) .
$$

By the second items of (6-17) and Lemma 6.8, the contribution to $\mathfrak{k}$ from $q_{\xi 0}\left(d\left(\chi-\chi_{g}\right)\right)$ is bounded in absolute value by $c_{0} r^{-1 / 2}\left(r^{-1 / 2}+\|\xi\|_{\infty}\right)\left(\|f\|_{\infty}+\|m\|_{\infty}\right)$.

Split the contribution to $\mathfrak{k}$ from $q_{\xi 0}\left(d \chi_{g}\right)$ into two parts by splitting $\chi_{g}$ as $x_{g 1}+x_{g 2}$, where $x_{g 2}$ is defined by using $\chi\left(r^{-1 / 2} \operatorname{dist}(x, \cdot)\right) g(x, \cdot)$ in (6-25) rather than $g(x, \cdot)$. The contribution to (6-24) from $d \chi_{g 1}$ is analyzed with the help of the third item in (6-17). The result finds this contribution bounded by $c_{0} r^{-1 / 2}\left(r^{-1 / 2}+\|\xi\|_{\infty}\right)\left(\|f\|_{\infty}+\|m\|_{\infty}\right)$.

The integral that is used to define $C$ 's component at $p$ of $t_{\xi}^{\dagger}\left(\left(r^{-1 / 2} q_{\xi 0}\left(d \chi_{g 2}\right), 0\right)\right)$ is analyzed as follows: First, the derivatives of $\chi_{g 2}$ that appear in $q_{\xi 0}\left(d \chi_{g 2}\right)$ that are in 
directions along the fiber of $\left.N\right|_{p}$ are transferred to $\chi_{C} q_{\xi 0}^{\dagger}\left(\theta_{1 \xi=0, r}\right)$ using integration by parts. This done, then the second item in (6-17) can be used to see that the resulting expression is bounded by $c_{0} r^{-1 / 2}\left(r^{-1 / 2}+\|\xi\|_{\infty}\right)\left(\|f\|_{\infty}+\|m\|_{\infty}\right)$.

The derivatives of $x_{g 2}$ which appear in $q_{\xi 0}\left(d x_{g 2}\right)$ and are transversal to the fiber of $N$ at $p$ are analyzed by exploiting the fact that the integral that defines $x_{g 2}$ is confined to regions where $\operatorname{dist}(x, \cdot) \leq r^{-1 / 2}$. In particular, the standard small distance approximations for $g(x, \cdot)$ can be employed when $r$ is large to write such a derivative - a component of $\left(\nabla^{H} g\right)(x, \cdot)-$ as a sum $\left(\partial / \partial z_{1}\right) \mathfrak{g}+\mathfrak{g}^{\prime}$ where the notation is as follows: First, $z=z_{1}+i z_{2}$ is a complex Gaussian coordinate along the fiber of $\left.N\right|_{p}$. Meanwhile, both $\mathfrak{g}$ and $\mathfrak{g}^{\prime}$ have the following properties: They are bounded by $c_{0} \operatorname{dist}(x, \cdot)^{-2}$ and their derivatives from the right are bounded in absolute value by $c_{0}\left(\operatorname{dist}(x, \cdot)^{-3}+r^{-1 / 2} \operatorname{dist}(x, \cdot)^{-2}\right)$.

These bounds are used to evaluate the integral of $\chi_{C} \theta_{1 \xi=0, r} q_{\xi 0}\left(d \chi_{g_{2}}\right)$ along the fiber of $\left.N\right|_{p}$ as follows: First, integrate by parts along the fiber of $N_{p}$ so as to transfer the $\partial / \partial z_{1}$ derivative on $\mathfrak{g}$ to a derivative along the fiber of $\chi_{C} q_{\xi 0}^{\dagger}\left(\theta_{1 \xi=0, r}\right)$. Having done this, the just mentioned bounds on the derivatives of $\mathfrak{g}$ and $\mathfrak{g}^{\prime}$ can be used in a straightforward way to bound the $q \xi_{0}\left(d \chi_{g}\right)$ contribution by $c_{0} r^{-1 / 2}\left(r^{-1 / 2}+\|\xi\|_{\infty}\right)\left(\|f\|_{\infty}+\|m\|_{\infty}\right)$.

Except for cosmetic changes, the analysis given can be employed so as to bound the other components of $\mathfrak{t}_{\xi}^{\dagger}(\mathfrak{f})$ by what is claimed in the first item of the lemma. A very similar sort of argument proves the claim made by the second item of the lemma. These are left to the reader.

Step 4 The $\xi=0$ version of $\mathfrak{q}$ is smooth; this follows from (6-21) using standard elliptic regularity theorems. More to the point, because the $L^{2}$ norm of $\mathfrak{q}$ on cylinders of the form $[s, s+1] \times M$ has an $s$-independent upper bound, there exists an $r-$ dependent constant $z$ such that the following is true: If $p \in \mathbb{R} \times M$ and $\rho \in(0,1]$, then

$$
\int_{\operatorname{dist}(p, \cdot)<\rho}\left(|\nabla \mathfrak{q}|^{2}+|\mathfrak{q}|^{2}\right) \leq z \rho^{4}
$$

Now write the $\xi=0$ version of $\mathfrak{q}$ as $\mathfrak{q}^{\perp}+t_{0}(\theta)$. It follows from what was just said about $\mathfrak{q}$ that each component of $\theta$ is also smooth; and it follows from (6-26) that $\|\theta\|_{\mathcal{K} *}$ is finite. In addition, what is said in Step 2 and what is said by Lemma 6.10 imply that $\|\theta\|_{\infty} \leq c_{0} r^{-1 / 2} \delta$ when $n$ is large.

Write $\theta=r^{-1 / 2} \lambda^{(0)}$; thus $\lambda^{(0)}$ is an element in $\mathcal{K}_{*}$ with $\left\|\lambda^{(0)}\right\|_{\infty} \leq c_{0} \delta$ if $n$ is large. Assume that such is the case. This $\lambda^{(0)}$ is the zero'th element of a sequence $\left\{\lambda^{(k)}\right\}_{k=0,1, \ldots}$ whose limit is the desired element $\xi$. The sequence is constructed 
inductively along the following lines: Suppose that $\lambda^{(k)} \in \mathcal{K}_{*}$ for some $k \geq 0$ and that $\left\|\lambda^{(k)}\right\|_{\infty}$ is such as to invoke Lemma 6.5. Assume as well that $\lambda^{(k)}$ is smooth. Use $\xi=\lambda^{(k)}$ to define $\left(A^{\xi}, \psi^{\xi}\right)$. This done, introduce the $\xi=\lambda^{(k)}$ version of $\mathfrak{q}=\mathfrak{b}(\xi)-\mathfrak{h}(\xi)$. Then $\mathfrak{q}\left(\lambda^{k}\right)$ is smooth, and the same argument that led to (6-26) in the case $k=0$ gives the analogous inequality for $\mathfrak{q}\left(\lambda^{k}\right)$ with perhaps a different $z$. Keeping this in mind, decompose $\mathfrak{q}\left(\lambda^{k}\right)=\mathfrak{q}^{\perp}+t_{\xi=\lambda(k)}(\theta)$. Then $\theta$ is smooth, and what with the $\mathfrak{q}\left(\lambda^{k}\right)$ version of (6-26), it follows that $\|\theta\|_{\mathcal{K}_{*}}$ is finite. Write this new version of $\theta$ is written as $r^{-1 / 2}\left(\lambda^{(k+1)}-\lambda^{(k)}\right)$, thus giving the next element in the sequence; provided that enough can be said about the size of $\left\|\lambda^{(k+1)}-\lambda^{(k)}\right\|_{\infty}$.

Step 5 To set up what is needed to construct the whole sequence $\left\{\lambda^{(k)}\right\}_{k=0,1, \ldots}$ and say something about its properties, suppose that $\xi$ and $\xi^{\prime}$ are in $\mathcal{K}_{*}$. It follows directly from the definitions of $t_{\xi}$ and $\left(c_{\xi}, \varsigma_{\xi}\right)$ using [9, (2-9), (2-11), (2-12)] that

$$
t_{\xi}^{\dagger}\left(\left(c_{\xi^{\prime}}, \varsigma \xi^{\prime}\right)\right)=r^{-1 / 2}\left(\xi^{\prime}+\mathfrak{e}\right)
$$

where

$$
\|\mathfrak{e}\|_{\infty} \leq c_{0}\left(r^{-1 / 2+8 \sigma}+\|\xi\|_{\infty}\right)\left\|\xi^{\prime}\right\|_{\infty} .
$$

By the same token, if $\xi$, $\xi^{\prime}$ and $\xi+\xi^{\prime}$ are in $\mathcal{K}_{*}$, then

$$
t_{\xi+\xi^{\prime}}^{\dagger}\left(\left(c_{\xi}, \varsigma_{\xi}\right)\right)-t_{\xi}^{\dagger}\left(\left(c_{\xi}, \varsigma_{\xi}\right)\right)=r^{-1 / 2} \mathfrak{e}
$$

where this new version of $\mathfrak{e}$ also obeys (6-28).

To continue, fix $\xi$ and $\xi^{\prime}$ as above, and use $s$ and $s^{\prime}$ to denote the functions that appear in their respective versions of (6-23). It follows from Lemma 6.7 that

$$
\left|s-s^{\prime}\right| \leq c_{0}\left\|\xi-\xi^{\prime}\right\|_{\infty}
$$

when $n$ is large. In addition, if $f$ is set equal to $\left(-r^{-1 / 2} d s,\left(e^{s}-1\right) \psi^{*}\right)$ and $f^{\prime}$ is defined analogously using $s^{\prime}$, it follows from Lemmas 6.7 and 6.10 that

$$
\left\|t_{\xi}^{\dagger}(f)-t_{\xi^{\prime}}^{\dagger}\left(f^{\prime}\right)\right\|_{\infty} \leq c_{0} \delta\left\|\xi-\xi^{\prime}\right\|_{\infty}
$$

when $n$ is large.

Step 6 Suppose now that $\lambda^{(k)}$ is as described in Step 4. Then (6-23) with (6-27)-(6-31) imply that

$$
\left\|\lambda^{(k+1)}-\lambda^{(k)}\right\|_{\infty} \leq c_{0} \delta\left\|\lambda^{(k)}\right\|_{\infty}
$$

if it is the case that $\left\|\lambda^{(k)}\right\|_{\infty} \leq c_{0} \delta$ and $n$ is sufficiently large. This has the following consequence: If $\delta \leq c_{0}^{-1}$ and if $n$ is sufficiently large, then the sequence $\left\{\lambda^{(k)}\right\}_{k=0,1, \ldots}$ 
can be constructed as described in Step 4. Moreover, (6-32) implies that this sequence converges in the $C^{0}$ topology. This is the topology that is defined by replacing each version of $\|\cdot\|_{\mathcal{K}}$ in $[9,(5-13)]$ with the norm $\|\cdot\|_{\infty}$.

Let $\xi$ denote this limit. Let $\|\cdot\|_{\mathcal{K}_{*}}$ denote the norm on $\mathcal{K}_{*}$ from [9, Step 4 in Section 5.a]. If $\|\xi\|_{\mathcal{K}_{*}}$ is finite, it then follows from the construction that $\mathfrak{b}(\xi)-\mathfrak{h}(\xi)$ is in $\mathbb{H} \frac{\perp}{\xi}$. This understood, the claim made by Lemma 6.9 follows with a proof that $\|\xi\|_{\mathcal{K}_{*}}$ is finite.

Step 7 To prove that $\|\xi\|_{\mathcal{K}_{*}}<\infty$, it is useful to introduce an equivalent norm and prove that the latter is finite on $\xi$. To this end, return for the moment to the context and notation of $[9,(2-27)]$. Fix $\varepsilon \in(0,1)$ and then define a new norm, denoted $\|\cdot\|_{\mathcal{K}, \varepsilon}$, on $C^{\infty}\left(C ; c^{*} V_{1,0} \mathfrak{C}_{E m}\right)$ by declaring

$$
\|\zeta\|_{\mathcal{K}, \varepsilon}^{2}=\sup _{p \in C, \rho<\varepsilon} \rho^{-v} \int_{\operatorname{dist}(\cdot, p)<\rho}|\nabla \zeta|^{2} .
$$

Replace $\|\cdot\|_{\mathcal{K}}$ in $[9,(5-13)]$ with $\|\cdot\|_{\mathcal{K}, \varepsilon}$, to define the norm $\|\cdot\|_{\mathcal{K}, \varepsilon}$, on $\mathcal{K}_{\text {smooth }}$. Note that $\|\cdot\|_{\mathcal{K}, \varepsilon}$ gives a norm on $\mathcal{K}_{\text {smooth }}$ that is equivalent to $\|\cdot\|_{\mathcal{K}}$.

The proof of convergence of $\left\{\lambda^{(k)}\right\}_{k=0,1,2, \ldots}$ with respect to the norm $\|\cdot\|_{\mathcal{K}, \varepsilon}$, requires first some remarks about $t_{\xi}\left(c_{\xi}, \varsigma_{\xi}\right)$. What is needed concerns the respective terms in (6-27) and (6-29) that are denoted by $\mathfrak{e}$. Here is what is needed about $\mathfrak{e}$ :

$$
\|\mathfrak{e}\|_{\mathcal{K}, \varepsilon} \leq c_{0}\left(r^{-1 / 2+8 \sigma}+\|\xi\|_{\infty}\right)\left\|\xi^{\prime}\right\|_{\mathcal{K}, \varepsilon}+c_{0}\|\xi\|_{\mathcal{K}, \varepsilon}\left\|\xi^{\prime}\right\|_{\infty}
$$

where $c_{0}$ is independent of $\varepsilon$. As with (6-29), this inequality follow readily from the definitions of $t_{\xi}$ and $\left(c_{\xi}, \varsigma_{\xi}\right)$ using [9, (2-9), (2-11), (2-12)].

The convergence of $\left\{\lambda^{(k)}\right\}_{k=0,1,2, \ldots}$ with respect to the norm $\|\cdot\|_{\mathcal{K}, \varepsilon}$ would follow directly from (6-27), (6-29) and (6-34) but for the contribution of the $\xi=\lambda^{(k)}$ version of $t_{\xi}\left(-r^{-1 / 2} d s,\left(e^{s}-1\right)\left(\psi^{*}+\varsigma \xi\right)\right)$ to the definition of $\lambda^{(k+1)}$. The next lemma supplies a fundamental bound for the $L^{2}$ norms of $d s$ and $\nabla d s$ that is used to control the contribution from this term. Here is the motivation for this lemma: If $\xi \in \mathcal{K}_{*}$ and if $\chi$ is any given $i \mathbb{R}$ valued function on $\mathbb{R} \times M$, then bounds on the $K$-norm of $t_{\xi}\left(-r^{-1 / 2} d \chi,\left(e^{\chi}-1\right)\left(\psi^{*}+\varsigma_{\xi}\right)\right)$ follow immediately from bounds on the $L^{2}$ norms of $d \chi$ and $\nabla d \chi$ on balls in $\mathbb{R} \times M$ of varying radii.

Lemma 6.11 There exists a constant $\kappa>0$ with the following significance: Fix $n>\kappa$ and set $r=r_{n}$. Suppose that $\varepsilon \in\left(0, r^{-1}\right]$ and that $\rho \in(0, \varepsilon]$. Let $\xi$ and $\xi^{\prime}$ denote two elements in $\mathcal{K}_{*}$ and let $s$ and $s^{\prime}$ denote the respective solutions to (6-13) given by the small normed fixed point of (6-16). Then

$$
\int_{\operatorname{dist}(p, \cdot) \leq \rho}\left(\left|\nabla d\left(s-s^{\prime}\right)\right|^{2}+\left|d\left(s-s^{\prime}\right)\right|^{2}\right) \leq c_{0} r^{-1 / 4} \rho^{v}\left(\left\|\xi-\xi^{\prime}\right\|_{\infty}^{2}+\left\|\xi-\xi^{\prime}\right\|_{\mathcal{K}, \varepsilon}^{2}\right) .
$$


This lemma is proved in Step 9.

To use this lemma to prove that $\xi \in \mathcal{K}_{*}$ when $n$ is large, set $\varepsilon=r^{-1}$. Given Lemma 6.11, it follows from (6-23), (6-27)-(6-29), and (6-34) that

(6-35) $\left\|\lambda^{(k+1)}-\lambda^{(k)}\right\|_{\mathcal{K}, \varepsilon} \leq c_{0}\left(r^{-1 / 4}+\delta\right)\left(\left\|\lambda^{(k)}-\lambda^{(k-1)}\right\|_{\infty}+\left\|\lambda^{(k)}-\lambda^{(k-1)}\right\|_{\mathcal{K}, \varepsilon}\right)$.

If $n$ is large, iteration of this inequality shows that the sequence $\left\{\lambda^{(k)}\right\}_{k=0,1, \ldots}$ is a Cauchy sequence with respect to the norm $\|\cdot\|_{\mathcal{K}, \varepsilon}$. As such it converges, and so its limit, $\xi$, has $\|\xi\|_{\mathcal{K}, \varepsilon}<\infty$. Thus, $\xi \in \mathcal{K}_{*}$ when $n$ is large.

Step 8 The proof of Lemma 6.11 requires an auxiliary lemma whose statement and proof occupy this Step 8. Here is the required lemma.

Lemma 6.12 The constant $\kappa$ in Lemma 6.7 and Lemma 6.10 can be chosen so that the following additional conclusion holds: Let $p$ and $p^{\prime}$ be any two points in $\mathbb{R} \times M$ and let $\Delta$ denote $r^{1 / 2} \operatorname{dist}\left(p, p^{\prime}\right)$. Then $\left|\chi(p)-\chi\left(p^{\prime}\right)\right| \leq \kappa \Delta(1+|\ln (\Delta)|)\left(\|f\|_{\infty}+\|m\|_{\infty}\right)$.

Proof of Lemma 6.12 The contribution to $\chi$ from $\mathrm{rm}$ has bounded derivative as can be seen using the second item of Lemma 6.8. In particular, this item of Lemma 6.8 gives a bound of the norm of this derivative by $c_{0} r^{1 / 2}\|m\|_{\infty}$. Thus, this part contributes at most $c_{0} \Delta\|m\|_{\infty}$ to $\left|\chi(p)-\chi\left(p^{\prime}\right)\right|$. To see about the contribution from $d^{\dagger} f$, write $G$ as in (6-18) using $\varepsilon=1$. This done, write the contribution from $d^{\dagger} f$ as $\chi_{g}+\left(\chi-\chi_{g}\right)$ where $\chi_{g}$ is defined by (6-25). It then follows using the second item of Lemma 6.10 and the second item of (6-17) that the function $\chi-\chi_{g}$ has bounded derivative with norm bounded by $c_{0} r^{1 / 2}\|f\|_{\infty}$. Thus, $\chi-\chi_{g}$ contributes at most $c_{0} \Delta\|f\|_{\infty}$ to $\left|\chi(p)-\chi\left(p^{\prime}\right)\right|$. Consider now the contribution from $\chi_{g}$. It proves convenient to write $\chi_{g}$ once again as $\chi_{g 1}+\chi_{g 2}$ where $\chi_{g 2}$ is defined by using $\chi(\sqrt{r} \operatorname{dist}(x, \cdot)) g(x, \cdot)$ in (6-35) rather than $g(x, \cdot)$. Integrate by parts to remove the derivative from $f$ and then appeal to the third and fourth items of (6-17) to see that $\chi_{g 1}$ has bounded derivative with norm bounded by $c_{0} r^{1 / 2}\|f\|_{\infty}$. Thus, $\chi_{g 1}$ contributes at most $c_{0} \Delta\|f\|_{\infty}$ to $\left|\chi(p)-\chi\left(p^{\prime}\right)\right|$. As for $\chi_{g 2}$, integrate by parts to put the derivatives from $f$ onto $g$. If $\operatorname{dist}\left(p, p^{\prime}\right)>c_{0}^{-1} r^{-1 / 2}$, use the second item in (6-17) to bound $\left|\chi_{g_{2}}(p)\right|$ and $\left|\chi_{g 2}\left(p^{\prime}\right)\right|$ both by $c_{0}\|f\|_{\infty}$ so as to conclude that $\left|\chi_{g 2}(p)-\chi_{g 2}\left(p^{\prime}\right)\right| \leq c_{0}\|f\|_{\infty} \leq c_{0} \Delta\|f\|_{\infty}$.

To consider the case where $\operatorname{dist}\left(p, p^{\prime}\right) \leq c_{0}^{-1} r^{-1 / 2}$, introduce Gaussian normal coordinates centered on the point midway between $p$ and $p^{\prime}$ so as to identify $p, p^{\prime}$ and the domain of integration as subsets of $\mathbb{R}^{4}$. Granted this identification, use the third item in (6-17) to bound $\left|g(p, y)-g\left(p^{\prime}, y\right)\right|$ by $c_{0} \operatorname{dist}\left(p, p^{\prime}\right)|y|^{-4}$; and use this to bound the contribution to $\left|\chi(p)-\chi^{\prime}\left(p^{\prime}\right)\right|$ from the portion of the integration domain 
where $|y| \geq 4 \operatorname{dist}\left(p, p^{\prime}\right)$. Meanwhile, use the second item in (6-17) to bound the integration over the rest of the integration domain by $c_{0} \operatorname{dist}\left(p, p^{\prime}\right)$. The bound using $\operatorname{dist}\left(p, p^{\prime}\right)|y|^{-4}$ supplies a term bounded by $c_{0} \Delta(1+|\ln (\Delta)|)\|f\|_{\infty}$.

Step 9 This step contains the following.

Proof of Lemma 6.11 To start the derivation of the needed estimates, fix two elements $\xi$ and $\xi^{\prime}$ in $\mathcal{K}_{*}$. By virtue of (6-13), the difference $s-s^{\prime}$ obeys an equation that can be written schematically as

$$
d^{\dagger} d\left(s-s^{\prime}\right)+2 r\left|\psi^{*}\right|^{2}\left(s-s^{\prime}\right)=\mathfrak{K} .
$$

Here, $\mathfrak{K}$ is the difference between the respective $\xi$ and $\xi^{\prime}$ versions of what is denoted in (6-13) by $-\mathfrak{R}+\mathfrak{P}_{b}+\mathfrak{P}_{\eta}$.

Fix $\rho \in\left(0, r^{-1 / 2}\right)$ and $p \in \mathbb{R} \times M$. Let $\chi_{p}$ denote the function on $\mathbb{R} \times M$ given by $\chi\left(\rho^{-1} \operatorname{dist}(\cdot, p)\right)$. Multiply both sides of (6-36) by $-\chi_{p}\left(s-s^{\prime}\right)$ and then integrate the result. What with (6-14), a suitable integration by parts and Holder's inequality finds that

$$
\begin{aligned}
\int_{\operatorname{dist}(p, \cdot) \leq \rho / 4}\left|d\left(s-s^{\prime}\right)\right|^{2} \leq c_{0} r \rho^{4}( & \left.\left\|s-s^{\prime}\right\|_{\infty}^{2}+\left\|\xi-\xi^{\prime}\right\|_{\infty}^{2}\right) \\
& +c_{0} \rho^{-2} \int_{\operatorname{dist}(p, \cdot) \leq \rho}\left|\left(s-s^{\prime}\right)-\overline{\left(s-s^{\prime}\right)}\right|^{2},
\end{aligned}
$$

where $\overline{s-s^{\prime}}$ denotes the average value of $s-s^{\prime}$ over the radius $\rho$ ball centered at $p$. Use (6-30) to bound $\left\|s-s^{\prime}\right\|_{\infty}$ by $c_{0}\left\|\xi-\xi^{\prime}\right\|_{\infty}$ and use Lemma 6.12 to bound the integral on the right in (6-37) by $c_{0}\left\|\xi-\xi^{\prime}\right\|_{\infty}^{2} r \rho^{6}\left(1+\left|\ln \left(\rho r^{1 / 2}\right)\right|^{2}\right)$. Here is the result:

$$
\int_{\operatorname{dist}(p, \cdot) \leq \rho / 4}\left|d\left(s-s^{\prime}\right)\right|^{2} \leq c_{0} r \rho^{4}\left(1+\left|\ln \left(\rho r^{1 / 2}\right)\right|^{2}\right)\left\|\xi-\xi^{\prime}\right\|_{\infty}^{2} .
$$

To continue, set $\varepsilon \in\left(0, r^{1 / 2}\right)$ and suppose now that $\rho \in(0, \varepsilon]$. It then follows from (6-14) that

$$
\int_{\mathbb{R} \times M} \chi_{p}|\mathfrak{K}|^{2} \leq c_{0} r^{2} \rho^{4}\left\|\xi-\xi^{\prime}\right\|_{\infty}^{2}+c_{0} r^{6 \sigma} \rho^{v}\left(r^{-1} \rho^{2}\right)\left\|\xi-\xi^{\prime}\right\|_{\mathcal{K}, \varepsilon}^{2} .
$$

Granted (6-39), multiply both sides of (6-36) by $\chi_{p}$ and then integrate the squares of the results. An integration by parts finds that

$$
\begin{array}{r}
\int_{\mathbb{R} \times M} \chi_{p}\left|\nabla d\left(s-s^{\prime}\right)\right|^{2} \leq c_{0} r^{2} \rho^{4}\left\|\xi-\xi^{\prime}\right\|_{\infty}^{2}+c_{0} r^{6 \sigma} \rho^{v}\left(r^{-1} \rho^{2}\right)\left\|\xi-\xi^{\prime}\right\|_{\mathcal{K}, \varepsilon}^{2} \\
+c_{0} \rho^{-2} \int_{\operatorname{dist}(p, \cdot) \leq \rho / 4}\left|d\left(s-s^{\prime}\right)\right|^{2}
\end{array}
$$


What with (6-38), this gives

$$
\begin{aligned}
\int_{\operatorname{dist}(p, \cdot) \leq \rho / 4}\left|\nabla d\left(s-s^{\prime}\right)\right|^{2} \leq & c_{0}\left(r \rho^{2}\left(1+\left|\ln \left(\rho r^{1 / 2}\right)\right|^{2}\right)\right. \\
& \left.+r^{6 \sigma} \rho^{v}\left(r^{-1} \rho^{2}\right)\right)\left(\left\|\xi-\xi^{\prime}\right\|_{\infty}^{2}+\left\|\xi-\xi^{\prime}\right\|_{\mathcal{K}, \varepsilon}^{2}\right) .
\end{aligned}
$$

Take $\varepsilon=r^{-1}$ and take $\rho \in(0, \varepsilon]$. The resulting versions of (6-38) and (6-41) imply what is claimed by the lemma.

This completes the proof of Lemma 6.9.

\section{6.e A bound for $\|\xi\|_{\mathcal{K}}$}

The purpose of this section is to prove that $\|\xi\|_{\mathcal{K}}$ is finite and small. The results are summarized by the following.

Lemma 6.13 The constant $\kappa$ in Lemma 6.9 can be chosen so that the following additional conclusion holds: The norm $\|\cdot\|_{\mathcal{K}_{1}^{2}}$ is finite on $\xi$ and $\|\xi\|_{\mathcal{K}_{1}^{2}} \leq \kappa \delta$.Thus, $\xi \in \mathcal{K}$. Moreover, $\|\xi\|_{\mathcal{K}} \leq \kappa \delta$.

Recall that the norm $\|\cdot\|_{\mathcal{K}_{1}^{2}}$ is defined by replacing each occurrence of $\|\cdot\|_{\mathcal{K}}$ in $[9$, $(5-13)]$ by the norm given by the top line in $[9,(2-27)]$.

Granted Lemma 6.13, the proof of Theorem 1.2 is completed as follows.

Proof of Theorem 1.2 If $n$ is large and so $\delta$ small, it is a consequence of Lemma 6.13 that $\xi$ obeys the assumptions that are made by [9, Proposition 6.4]. This understood, (6-21) and Lemma 6.9 imply that $\mathfrak{q}=\mathfrak{q}(\xi)-\mathfrak{h}(\xi)$ as described above is precisely the element in $\mathbb{H}$ that is assigned to $\xi$ by [9, Proposition 6.4]. As a consequence, $\xi$ is given by [9, Proposition 7.1] if $n$ is large and so $\delta$ is small. Indeed, [9, Proposition 7.1] asserts that there is unique, small normed solution to [9, (7-1)] with any given small normed value for $q(\cdot)$. Let $\lambda=q(\xi)$. For each $x \in[0,1]$, [9, Proposition 7.1] finds a solution to $\xi_{x}$ to (7-1) with $q\left(\xi_{x}\right)=x \lambda$. This gives a continuous path in $\left(A_{n}, \psi_{n}\right)$ 's component of $\mathcal{M}_{1}\left(\mathfrak{c}_{-}, \mathfrak{c}_{+}\right)$that connects the pair $\left(A_{n}, \psi_{n}\right)$ to a point in the image of $\Psi^{r}$. Because $\Psi^{r}$ maps any given component of $\mathcal{M}_{1}\left(\Theta_{-}, \Theta_{+}\right)$onto a component of $\mathcal{M}_{1}\left(\mathfrak{c}_{-}, \mathfrak{c}_{+}\right)$, this implies that $\left(A_{n}, \psi_{n}\right)$ is in the image of $\Psi^{r}$. However, this conclusion contradicts the assumption made at the outset. This contradiction proves Theorem 1.2. 
Proof of Lemma 6.13 Reintroduce the constant $R_{*}$ given in [9, (4-8)]. Fix $T>$ $100 R_{*}$ so as to define the bump function, $m$, on $\mathbb{R} \times M$. This function is to equal 1 where $|s| \leq T$ and to vanish where $|s| \geq T+r^{2}$. Also, $|d m| \leq c_{0} r^{-2}$. To be precise, $m$ is defined to equal $\chi\left(r^{-2}(|s|-T)\right)$ where the $|s|>T$ and where the distance to any Reeb orbit from either $\Xi_{\Sigma_{-}}$or $\Xi_{\Sigma_{+}}$is greater than $100 \rho_{*}$. To define $m$ where $s<-T$ and where the distance to $\gamma \in \Xi_{\Sigma-}$ is less than $100 \rho_{*}$, introduce the coordinates $(w, t, z)$ as in [9, (4-1)]. This done, set $m$ on the domain in question to be $\chi\left(\left.r^{-2}\left(\ell_{\gamma} /(2 \pi)\right)|w+\chi(1 / 2)| z\right|^{2} \mid-T\right)$ where $\chi=\left(1-\chi\left(|z| /\left(16 \rho_{*}\right)\right)\right.$. Use the same formula for $m$ at points where both $s>T$ and the distance to any given $\gamma \in \Xi_{\Sigma+}$ less than $100 \rho_{*}$.

Introduce $\xi_{T}$ to denote the element in $K$ whose $C \in \mathcal{C}$ entries are those of $\xi$, and whose respective $\gamma \in \Xi_{\Sigma-}$ and $\gamma \in \Xi_{\Sigma+}$ entries are given in terms of the coordinates in [9, (4-1)] by $\chi\left(\left(\ell_{\gamma} /(2 \pi)\right) r^{-2}|w|-T\right) \xi_{\gamma \pm}$. The first four parts of what follows

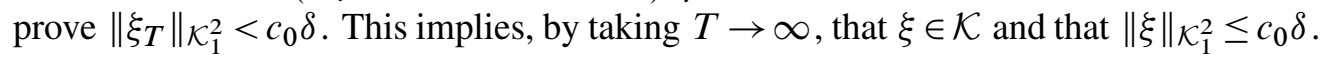
Part 5 of what follows uses this last bound to prove that $\|\xi\|_{\mathcal{K}} \leq c_{0} \delta$.

Part 1 Set $\mathfrak{q}_{T}=m \mathfrak{q}$. Note that $\mathfrak{q}_{T} \in \mathbb{H}_{\xi}^{\perp}$ because $T \gg R$. What with the top line of (6-21), this $\mathfrak{q}_{T}$ obeys the equation

$$
\mathfrak{D} \mathfrak{q}_{T}=-r^{-2} m^{\prime} \mathfrak{q}_{T} \mathfrak{q}-r^{1 / 2} \mathfrak{q}_{T} \mathfrak{q} * \mathfrak{q}_{T}-2 r^{1 / 2} \mathfrak{h} * \mathfrak{q}_{T}+r^{-1 / 2} m\left(\mathfrak{v}-\mathfrak{v}_{\mathfrak{h}}\right),
$$

where $m^{\prime}$ is the endomorphism of $i T^{*} R \oplus \mathbb{S} \oplus i \mathbb{R}$ that is defined by applying the principle symbol of $\mathfrak{D}$ to the 1 -form $d m$. This equation is used to derive the upper bound

$$
\left\|\mathfrak{q}_{T}\right\|_{\mathbb{H}} \leq c_{0} r^{-1 / 2}\left(r^{-1 / 2+8 \sigma}+\left\|\xi_{T}\right\|_{\mathcal{K}_{1}^{2}}\right)
$$

Such an upper bound follows from what is said in [9, Lemma 6.1] given suitable bounds for the $L^{2}$ norms of the various terms that appear on the right hand side of (6-42). The desired bounds are derived in the next paragraph.

Given what is said in the second item of (6-21), it follows that the $L^{2}$ norm of the term $r^{-2} m^{\prime} \mathfrak{q}$ is bounded by $c_{0} r^{-1} \delta$. To say something about the norm of $r^{1 / 2} \mathfrak{q} * \mathfrak{q}_{T}$, use the fact that bilinear operator $(\cdot) *(\cdot)$ involves the spinor component of at least one of its entries to see that the $L^{2}$ norm of this term is no greater than $c_{0} \delta\left\|_{T}\right\|_{\mathbb{H}}$. Given what is said about $\mathfrak{h}$ in [9, Lemma 6.3], it follows that the $L^{2}$ norm of $r^{1 / 2} \mathfrak{h} * \mathfrak{q}_{T}$ is less than $c_{0} \delta\left\|\mathfrak{q}_{T}\right\|_{\mathbb{H}}$ when $n$ is large. The arguments that are used to prove the third item in [9, Lemma 6.3] bound the $L^{2}$ norm of $r^{-1 / 2} m\left(\mathfrak{v}-\mathfrak{v}_{\mathfrak{h}}\right)$ by $c_{0} r^{-1 / 2}\left(r^{-1 / 2+8 \sigma}+r^{-1} \delta+\left\|\xi_{T}\right\|_{\mathcal{K}_{1}^{2}}\right)$. 
Part 2 Because $T \gg R$, the term $-r^{-1} m^{\prime} \mathfrak{q}$ that appears in (6-42) is in the subspace $\mathbb{L}_{\xi}^{\frac{1}{\xi}}=\left(1-\Pi_{\xi}\right) \mathbb{L}$. This understood, (6-42) requires that

$$
\Pi_{\xi}\left(m\left(\mathfrak{v}-\mathfrak{v}_{\mathfrak{h}}\right)\right)=r^{1 / 2} \Pi_{\xi}\left(\mathfrak{D} \mathfrak{q}_{T}+r^{1 / 2} \mathfrak{q} * \mathfrak{q}_{T}+2 r^{1 / 2} \mathfrak{h} * \mathfrak{q}_{T}\right) .
$$

A bound on the $\mathcal{K}_{1}^{2}$ norm of $\xi$ will be derived using (6-44). This task requires the derivation of bounds on the $L^{2}$ norms of the terms that appear on the right hand side of this equation.

The easiest of the terms to consider are $r \Pi_{\xi}\left(\mathfrak{q} * \mathfrak{q}_{T}\right)$ and $r \Pi_{\xi}\left(\mathfrak{h} * \mathfrak{q}_{T}\right)$. Indeed, because $(\cdot) *(\cdot)$ involves the spinor component of at least one of its entries, it follows that the $L^{2}$ norm of $r \Pi_{\xi}\left(\mathfrak{q} * \mathfrak{q}_{T}\right)$ is bounded by $c_{0} r^{1 / 2} \delta\left\|\mathfrak{q}_{T}\right\|_{\mathbb{H}}$; thus by $c_{0} \delta\left(r^{-1 / 2+8 \sigma}+\left\|\xi_{T}\right\|_{\mathcal{K}_{1}^{2}}\right)$. Meanwhile, it follows from the second item of [9, Lemma 6.3] that the $L^{2}$ norm of $r \Pi_{\xi}\left(\mathfrak{h} * \mathfrak{q}_{T}\right)$ is bounded by $c_{0} r^{-1 / 16}\left(r^{-1 / 2+8 \sigma}+\|\xi\|_{\mathcal{K}_{1}^{2}}\right)$.

It follows from [9, (6-6), (6-7)] that the $L^{2}$ norm of $r^{1 / 2} \Pi_{\xi} \mathfrak{D q} \mathfrak{q}_{T}$ obeys

$$
r^{1 / 2}\left\|\Pi_{\xi} \mathfrak{D q}_{T}\right\|_{2} \leq c_{0}\left(\left\|\xi_{T}\right\|_{\mathcal{K}_{1}^{2}}+r^{-1}\right)(r \mathcal{W})^{1 / 2}+c_{0} \rho_{*} r^{1 / 2}\left\|\mathfrak{q}_{T}\right\|_{\mathbb{H}},
$$

where the notation is as follows: What is denoted as $\mathcal{W}$ is given by

$$
\mathcal{W}=\sup _{(C, m) \in \vartheta} \sup _{p \in C}\left|\int_{\left.N\right|_{p}} \mathcal{k}\left(\mathfrak{q}_{T}\right)\right|^{2},
$$

with $K\left(\mathfrak{q}_{T}\right)$ being a linear function of the components of $\mathfrak{q}_{T}$ with support on the part of the fiber of $N$ at $p$ where the distance to $p$ is bounded by $\rho_{*}$. Moreover, this linear function $\mathcal{K}$ obeys $|\mathcal{K}|+r^{-1 / 2}\left|\nabla^{V} \mathcal{K}\right| \leq c_{0} r^{1 / 2} e^{-\sqrt{r} \operatorname{dist}(\cdot, \Sigma) / c_{0}}$ with $\Sigma$ denoting $\bigcup_{(C, m) \in \vartheta} C$ and $\nabla^{V}$ denotes differentiation along the fiber of $N$. By way of explanation, the term with $\mathcal{W}$ is the analog here of the contribution to [9, (6-66)] of what is called $\mathcal{A}_{1}$ in $[9,(6-67)]$. Meanwhile, the term $c_{0} \rho_{*} r^{1 / 2}\left\|\mathfrak{q}_{T}\right\|_{\mathbb{H}}^{2}$ in (6-45) corresponds to $r^{1 / 2}$ times what is denoted in [9, (6-67)] by $\mathcal{A}_{2}$. As is explained in Part 3, W $\leq c_{0} r^{-1} \delta^{2}$. Granted this, it then follows from (6-45) and what is said in the preceding paragraph that the $L^{2}$ norm of the right hand side of (6-44) is bounded by

$$
c_{0} \delta\left(r^{-1 / 2+8 \sigma}+\left\|\xi_{T}\right\|_{\mathcal{K}_{1}^{2}}\right)
$$

when $n$ is large.

Part 3 To see why $\mathcal{W} \leq c_{0} r^{-1} \delta^{2}$, consider first the contribution from $\mathfrak{h}(\xi)$. This section of $i T^{*} M \oplus \mathbb{S}$ has a part whose absolute value is less than $c_{0}\left(r^{-1}+\right.$ $\left.r^{-1 / 2} e^{-\sqrt{r} \operatorname{dist}(\cdot, \Sigma) / c_{0}}\right)$, and a second part that is the product of a function with support where $|s| \geq R_{*}$ and something invariant with respect to translations on the $\mathbb{R}$ factor of $\mathbb{R} \times M$ with $\|\cdot\|_{\mathbb{H}}$ norm bounded by $c_{0} r^{-1}$ on any given constant $s$ slice of $\mathbb{R} \times M$. 
The bounded part contributes at most $r^{-2}$ to $\mathcal{W}$. The other part has support far out on the ends of the curves in $\mathcal{C}$. This part comes from what is denoted in $[9,(6-30)]$ by $\mathfrak{b}_{-}$ and $\mathfrak{b}_{+} ;$it corresponds to what is denoted by $\mathfrak{e}_{\zeta}$ in [9, Lemma 3.10]. Given that each end of each $C$ from a pair in $\vartheta$ is transversal to the constant $s$ slices of $\mathbb{R} \times M$, it follows using the fact that $L_{1}^{2}$ functions restrict to hyperplanes as $L^{2}$ functions that the integral of either the $\mathfrak{b}_{-}$or $\mathfrak{b}_{+}$version of $\left|\mathfrak{e}_{\xi}\right|^{2}$ over what is the normal bundle fiber $\left.N\right|_{p}$ is bounded by a uniform multiple of $\left\|\mathfrak{e}_{\xi}\right\|_{\mathbb{H}}^{2}$. As this is bounded by $c_{0} r^{-2}$, it follows that the contribution to $\mathcal{W}$ from $\mathfrak{h}(\xi)$ is bounded by $c_{0} r^{-2}$.

Turn now to the contribution to $\mathcal{W}$ from $\mathfrak{b}(\xi)$. As can be seen in (6-12), all but the contribution of $r^{-1 / 2} d s$ to $\mathfrak{b}(\xi)$ is bounded by $c_{0} \delta$. As a consequence, all but $r^{-1 / 2} d s$ contribute at most a factor $c_{0} r^{-1} \delta^{2}$ to $\mathcal{W}$. A bound by $c_{0} r^{-1} \delta^{2}$ on the contribution to $\mathcal{W}$ from $r^{-1 / 2} d s$ follows as an immediate consequence of the upcoming Lemma 6.14.

Lemma 6.14 augments the assertions that are made by Lemmas 6.7, 6.10 and 6.12. To set the notation used by the lemma, suppose that $D \subset \mathbb{C}$ is a disk centered at the origin and that $\varphi: D \rightarrow \mathbb{R} \times M$ is a smooth embedding. Suppose that $w$ is a section of $\varphi^{*} T(\mathbb{R} \times M)$ and $a$ is a 1 -form on $\mathbb{R} \times M$. Then $\langle a, w\rangle$ is used to denote the function on $D$ that is obtained by viewing $w$ as a section along $\varphi(D)$ of $T(\mathbb{R} \times M)$ and then pairing the latter at any given point in $D$ with the 1 -form $a$.

Lemma 6.14 Fix a constant $c \geq 1$ and the constant $\kappa$ that appears in Lemmas 6.7, 6.10 and 6.12 can be chosen so that the following additional conclusion hold: Let $D \subset \mathbb{C}$ denote the disk of radius $\kappa^{-1}$ centered at the origin. Suppose that $\varphi: D \rightarrow \mathbb{R} \times M$ is an embedding that is isometric at the origin in $D$ and whose second derivatives are bounded by $c$. Let $o$ denote a smooth section of $\varphi^{*} T(\mathbb{R} \times M)$ with compact support in the concentric disk of with half $D$ 's radius. Then

$$
\left|\int_{D}\langle d \chi, o\rangle\right| \leq \kappa\left(\|f\|_{\infty}+\|m\|_{\infty}\right) \int_{D}\left(|\nabla o|+r^{1 / 2}|o|\right) .
$$

Proof of Lemma 6.14 The arguments are much the same as those used in the proof of the first item of Lemma 6.10. In short, write the Green's function $G$ as in (6-18) for the case $\varepsilon=1$ and define $\chi_{g}$ as in (6-25). Given the second items in (6-17) and Lemma 6.8, the contribution to the integral of $\langle d \chi, o\rangle$ from $d\left(\chi-\chi_{g}\right)$ is bounded by

$$
c_{0}\left(\|f\|_{\infty}+\|m\|_{\infty}\right) r^{1 / 2} \int_{D}|o| .
$$

Meanwhile, the contribution from $\left\langle d \chi_{g}, o\right\rangle$ is bounded by writing $\chi$ again as $\chi_{g 1}+\chi_{g 2}$. The contribution from $\left\langle d \chi_{g 1}, o\right\rangle$ is also bounded by (6-48) as can be seen using the second and third items in (6-17). The contribution of $\left\langle d \chi_{g 1}, o\right\rangle$ is bounded by 
$c_{0}\left(\|f\|_{\infty}+\|m\|_{\infty}\right)$ times the $L^{1}$ norm of $\nabla q$. To see this, integrate by parts to move derivatives of $x$ along $\varphi(D)$ onto $o$ and then use the second item in (6-17). To handle the term with a derivative of $\chi$ that is transverse to $D$, use the standard short distance expansion of $g(x, \cdot)$ to write any such derivative of $g$ as $\left(\partial / \partial z_{1}\right) \mathfrak{g}+\mathfrak{g}^{\prime}$, where $z=z_{1}+i z_{2}$ a complex Gaussian coordinate along $\varphi(D)$, and where $\mathfrak{g}$ and $\mathfrak{g}^{\prime}$ are both bounded by $c_{0} \operatorname{dist}(x, \cdot)^{-2}$ and have derivatives from the right bounded in absolute value by $c_{0}\left(\operatorname{dist}(x, \cdot)^{-3}+r^{-1 / 2} \operatorname{dist}(x, \cdot)^{-2}\right)$.

Part 4 This part of the proof begins with a description of the left hand side of (6-44). In this regard, much can be borrowed from what is done in [9, Section 7.d]. To this end, write $\Pi_{\xi}\left(\mathfrak{v}-\mathfrak{v}_{\mathfrak{h}}\right)$ as $\left.\theta=\left(\left(\theta_{C}\right)_{(C, m) \in \vartheta},\left(\theta_{\gamma-}\right)_{\gamma \in \Xi_{\Sigma-}}\right),\left(\theta_{\gamma+}\right)_{\gamma \in \Xi_{\Sigma+}}\right)$. The various components of $\theta$ are described by [9, (7-30), (7-31), (7-34)].

Let $\mathcal{T}_{1}$ denote the linear map from $\mathcal{K}$ to $\mathcal{L}$ that is defined in [9, Part 2 of Section 7.e]. By virtue of what was said above about $\Pi_{\xi}\left(\mathfrak{v}-\mathfrak{v}_{\mathfrak{h}}\right)$, it follows from (6-44) and (6-47) that $\xi_{T}$ obeys an equation of the form

$$
\mathcal{T}_{1}\left(\xi_{T}\right)+e_{1}\left(\xi_{T}\right)=e_{0 T},
$$

where $e_{1}$ obeys $\left\|e_{1}\left(\xi_{T}\right)\right\|_{L^{2}} \leq c_{0} \delta\left\|\xi_{T}\right\|_{\mathcal{K}_{1}^{2}}$ and $e_{0}$ obeys $\left\|e_{0}\right\|_{L^{2}} \leq c_{0} r^{-1 / 2+8 \sigma}$. To elaborate, the terms $\mathcal{T}_{1}\left(\xi_{T}\right)+e_{1}\left(\xi_{T}\right)$ account for the difference between the $\xi_{T}$ version of $\Pi_{(\cdot)}\left(\mathfrak{v}-\mathfrak{v}_{\mathfrak{h}}\right)$ and the version that is defined by replacing $\xi$ with $0 \in \mathcal{K}$.

Let $C \in \mathcal{C}$ denote the one element that is not $\mathbb{R}$-invariant, and use $V_{0}$ to denote the 1-dimensional vector $L^{2}$ kernel of $\mathcal{D}_{C}$ with $\mathcal{D}_{C}$ defined as in [9, (4-5)]. A linear map $q: \mathcal{K} \rightarrow V_{0}$ is described in [9, Section 7.f]. According to [9, Proposition 7.2], the operator $\mathcal{T}_{1}$ maps the kernel of $q$ surjectively onto $\mathcal{L}$. As is explained in the next paragraph, $\xi_{T}$ can be written as $\xi_{T *}+\lambda_{T}$ where $q\left(\xi_{T *}\right)=0$ and where

$$
\left\|\lambda_{T}\right\|_{\mathcal{K}} \leq c_{0} \delta \quad \text { and } \quad\left\|\mathcal{T}_{1}\left(\lambda_{T}\right)\right\|_{\mathcal{L}} \leq c_{0} \delta r^{-1 / c_{0}} .
$$

Granted this decomposition, it follows from (a) and (b) of the second item of [9, Proposition 7.2] that $\left\|\xi_{T *}\right\|_{\mathcal{K}_{1}^{2}} \leq c_{0}\left(r^{-1 / 2+8 \sigma}+\delta r^{-1 / c_{0}}\right)$. These bounds on $\xi_{T *}$ and $h_{T}$ imply what is claimed by Lemma 6.13 .

To obtain $\lambda_{T}$, reintroduce the linear map $q^{*}: V_{0} \rightarrow K$ that is defined at the end of [9, Section 7.f]. Let $C$ again denote the one surface from a pair in $\vartheta$ that is not $\mathbb{R}-$ invariant. Fix an element $o \in V_{0}=\operatorname{kernel}\left(\mathcal{D}_{C}\right)$ with $L^{2}$ norm 1 . Then $\lambda_{T}=r q^{*}(\mathrm{o})$ where $r=\left\langle q\left(\xi_{T}\right), \mathrm{o}\right\rangle_{2}\left\langle q q^{*}(\mathrm{o}), \mathrm{o}\right\rangle_{2}^{-1}$ Here, $\langle$,$\rangle denotes the L^{2}$ inner product on the space of square integrable sections of $C$ 's normal bundle. Note in this regard that $\left\langle q q^{*}(\mathrm{o}), \mathrm{o}\right\rangle_{2}$ differs from 1 by at most $c_{0} r^{-1 / c_{0}}$ The asserted bounds on the norms of $\lambda_{T}$ and $\mathcal{T}_{1}\left(\lambda_{T}\right)$ follow directly from what is said about $q^{*}(\mathrm{o})$ in the paragraph following [9, (7-49)]. 
Part 5 Granted that $\xi \in \mathcal{K}$, it satisfies the $T \rightarrow \infty$ version of what is written in (6-49), this the equation $\mathcal{T}_{1}(\xi)+e_{1}(\xi)=e_{0}$. It is a consequence of [9, (7-30), (7-31) and (7-34)] that $\left\|e_{1}(\xi)\right\|_{\mathcal{K}} \leq c_{0} \delta\|\xi\|_{\mathcal{K}}$. Given what is said in (6-49) about $\lambda_{T}$, it follows from (c) of the second item in [9, Proposition 7.2] that $\|\xi\|_{\mathcal{K}} \leq c_{0}\left(\delta+\left\|e_{0}\right\|_{\mathcal{L}}\right)$. Thus, the desired bound on $\|\xi\|_{\mathcal{K}}$ follows from a bound on $\left\|e_{0}\right\|_{\mathcal{L}}$ by $c_{0}\left(r^{-1 / 4}+\delta\|\xi\|_{\mathcal{K}}\right)$. Such a bound is derived in the three steps that follow.

Step 1 Define the norm $\|\cdot\|_{*}$ as in $[9,(6-51)]$. The derivation of the desired $\left\|e_{0}\right\|_{\mathcal{L}}$ bound requires the bound

$$
\|\mathfrak{q}\|_{*} \leq c_{0} r^{-1 / 2}\left(\delta+\|\xi\|_{\mathcal{K}}\right) .
$$

This bound follows from [9, Lemma 6.5] with the top equation in (6-21) playing the role played by [9, (6-55)]. Note that the assumptions for [9, Lemma 6.5] hold when $n$ is large by virtue of the following facts: First, (6-43) implies that $\mathfrak{q}$ is in $\mathbb{H}$ and that

$$
\|\mathfrak{q}\|_{\mathbb{H}} \leq c_{0} r^{-1 / 2} \delta
$$

Meanwhile, it follows from what is said in the preceding parts of the proof that the square of the $L^{2}$ norm of $\mathfrak{u}=r^{-1 / 2}\left(\mathfrak{v}-\mathfrak{v}_{\mathfrak{h}}\right)$ is bounded by $\mathfrak{m}_{\mathbb{L}}=c_{0} r^{-1} \delta^{2}$. In addition, what is said in $\left[9\right.$, Section 7.d] implies that the $\mathfrak{u}=r^{-1 / 2}\left(\mathfrak{v}-\mathfrak{v}_{\mathfrak{h}}\right)$ version of the integral on the right hand side of $[9,(6-56)]$ is bounded by $\mathfrak{m}_{*}=c_{0} r^{-1}\left(r^{-1 / 2+8 \sigma}+\|\xi\|_{\mathcal{K}}\right)^{2}$.

Step 2 Return to the notation used in [9, Section 7.b] and Part 2 of the digression that follows [9, Lemma 6.5]. In particular, fix $\rho \in(0,1)$ and let $D_{\rho}$ denote a disk of radius $\rho$ as described in Part 2 of this same digression. If $D_{\rho}$ is in some $(C, m) \in \vartheta$ version of $C-\left(\bigcup_{\mathcal{E} \in \mathcal{E}_{C}} \mathcal{E}_{2 R}\right)$, use $e_{0 *}$ to denote the component $e_{0 C}$. By the same token, if $D_{\rho}$ is in some $U_{\gamma-}$ version of $(-\infty,-R] \times S^{1}$, or in some $U_{\gamma+\text { version of }}$ $[R, \infty) \times S^{1}$, use $e_{0 *}$ to denote the corresponding component of $e_{0}$.

Step 3 The $L^{2}$ norm of $e_{0 *}$ over $D_{\rho}$ has a contribution from the $\xi=0$ version of $\Pi_{(.)}\left(\mathfrak{v}-\mathfrak{v}_{\mathfrak{h}}\right)$ and a contribution from each term in $r^{1 / 2} \Pi_{\xi}\left(\mathfrak{D q}+r^{1 / 2} \mathfrak{q} * \mathfrak{q}+2 r^{1 / 2} \mathfrak{h} * \mathfrak{q}\right)$. It is a direct consequence of [9, (7-30), (7-31) and (7-34)] that the contribution from the $\xi=0$ version of $\Pi_{(\cdot)}\left(\mathfrak{v}-\mathfrak{v}_{\mathfrak{h}}\right)$ is no greater than $c_{0} r^{-1 / 2+8 \sigma} \rho$.

Turn now to the contribution from $r^{1 / 2} \Pi_{\xi} \mathfrak{D q}$. As noted in [9, Step 2 of Section 7.b], the square of the $L^{2}$ norm of the relevant component of $\Pi_{\xi} \mathfrak{D} q$ over $D_{\rho}$ is at most $c_{0}\left(\mathcal{A}_{1}+\mathcal{A}_{2}\right)$ with the latter defined in [9, (6-67)]. [9, (6-68)] bounds $\mathcal{A}_{1}$ by $\mathfrak{m}_{\mathbb{L}}=r^{-1}\left(r^{-1 / 2+8 \sigma}+\|\xi\|_{\mathcal{K}_{1}^{2}}\right)^{2}$. As $\|\xi\|_{\mathcal{K}_{1}^{2}} \leq c_{0} \delta$, this finds $\mathcal{A}_{1} \leq c_{0} r^{-1} \delta^{2}\|\xi\|_{\mathcal{K}}^{2} \rho^{v}$.

A bound on $\mathcal{A}_{2}$ for the case $\rho>r^{-1 / 2-v}$ is given in [9, (6-69)] using this same $\mathfrak{m}_{\mathbb{L}}$. This bound finds the $\rho>r^{-1 / 2-v}$ version of $\mathcal{A}_{2}$ to be less than $c_{0} r^{-3 / 2} \delta^{2} \rho^{v}$. A bound 
on the $\rho<r^{-1 / 2}$ versions of $\mathcal{A}_{2}$ by $c_{0} r^{-3 / 2}\left(\delta+\|\xi\|_{\mathcal{K}}\right)^{2}$ is obtained by invoking [9, Lemma 7.4] using the version of [9, (6-55)] that is given by the top line in (6-21). Note in this regard that $\|\mathfrak{q}\|_{*}$ in this case is given in (6-51) and $\mathfrak{m}_{*}=c_{0} r^{-1}\left(r^{-1 / 2+8 \sigma}+\|\xi\|_{\mathcal{K}}\right)^{2}$.

Consider next the contributions from $r \Pi_{\xi}(\mathfrak{h} * \mathfrak{q})$ and $r \Pi_{\xi}(\mathfrak{q} * \mathfrak{q})$ to the square of the $L^{2}$ norm of $e_{0 *}$ over $D_{\rho}$. To this end, the arguments used in [9, Step 3 of Section 7.b] can be applied with but minor notational changes to bound these contributions. To elaborate, $[9,(7-6)]$ implies that $r \Pi_{\xi}(\mathfrak{h} * \mathfrak{q})$ contributes $c_{0} r^{-1 / 8}\left(r^{-1 / 2+8 \sigma}+\|\xi\|_{\mathcal{K}}\right) \rho^{v}$ or less to the $L^{2}$ norm of $e_{0 *}$ over $D_{\rho}$. Meanwhile, [9, (7-12)] with the bound $\|\xi\|_{\mathcal{K}_{1}^{2} \leq c_{0} \delta}$ implies that the contribution of $r \Pi_{\xi}(q * q)$ to the $L^{2}$ norm of $e_{0 *}$ over $D_{\rho}$ can be no greater than $c_{0} \delta\left(r^{-1 / 2+8 \sigma}+\|\xi\|_{\mathcal{K}}\right) \rho^{v}$.

Taken together, these bounds imply that $\left\|e_{0}\right\|_{\mathcal{L}} \leq c_{0}\left(r^{-1 / 4}+\delta\|\xi\|_{\mathcal{K}}\right)$ as claimed.

\section{Proof of Lemma 6.2}

The arguments for Lemma 6.2 are much like those in Sections $5 \mathrm{~b}-5 \mathrm{~d}$ of the article $\mathrm{SW}=\mathrm{Gr}$ in [6]. Note in this regard that the author found a gap in the proof of the latter's Lemma 5.5 while writing this subsection. What follows has a replacement lemma that is used with the subsequent arguments from the article $\mathrm{SW}=\mathrm{Gr}$ in [6] to prove Lemma 6.2. This replacement lemma can be used as well in the article $\mathrm{SW}=\mathrm{Gr}$ of [6] in lieu of the latter's Lemma 5.5.

The proof of Lemma 6.2 starts by assuming that the lemma is false and then proceeds to derive nonsense from this assumption. To begin this derivation, introduce

$$
\begin{aligned}
& \delta_{n}=r_{n}^{1 / 2} \sup _{z \in \alpha_{n}^{-1}(0)} \operatorname{dist}\left(\bigcup_{(C, m) \in \vartheta} C, z\right) \\
& \delta_{n}^{\prime}=r_{n}^{1 / 2} \sup _{z \in \cup_{(C, m) \in \vartheta} C} \operatorname{dist}\left(z, \alpha_{n}^{-1}(0)\right) .
\end{aligned}
$$

If the lemma is false, then there is a subsequence of $\left\{\left(r_{n},\left(A_{n}, \psi_{n}\right)\right)\right\}_{n=1,2, \ldots}$, hence relabeled consecutively from 1 , such that either $\left\{\delta_{n}\right\}_{n=1,2, \ldots}$ is bounded away from zero, or $\left\{\delta_{n}^{\prime}\right\}_{n=1,2, \ldots}$ is bounded away from zero. As is explained in Section 7.a, it is always the case the any sufficiently large $n$ version of $\delta_{n}^{\prime}$ is no greater than $c_{0} \delta_{n}$. Thus, the convergence of the former sequence implies the convergence of the latter. This understood, assume that there exists $\delta_{0}>0$ such that $\delta_{n}>\delta_{0}$ for all $n$. Sections 7.b-7.e derive the desired nonsense from this assumption. 


\section{7.a $\delta_{n}$ versus $\delta_{n}^{\prime}$}

What follows explains why $\delta_{n}^{\prime}<c_{0} \delta_{n}$ for $n$ large. If $(C, m) \in \vartheta$ and let $\pi: N \rightarrow C$ denote as usual $C$ 's normal bundle. Fix $\delta>0$ and let $N_{\delta} \rightarrow C$ denote the radius $\delta$ subdisk bundle. Lemma 6.1 guarantees that all sufficiently large $n$ versions of $\alpha_{n}^{-1}(0)$ intersect $e_{C}\left(\left.N_{\delta}\right|_{z}\right)$ for each $z \in C$. Furthermore, no point in $\alpha_{n}^{-1}(0)$ lies outside $\bigcup_{(C, m) \in \vartheta} e_{C}\left(N_{2 \delta}\right)$.

Fix $(C, m) \in \vartheta$ and $T \gg R$ so that the $|s|>T$ part of $C$ is far out on each end of $C$. If $n$ is large, and $z \in C$ lies where $|s|<T$, then $\operatorname{dist}\left(z, \alpha_{n}^{-1}(0)\right)<\delta_{n} r_{n}^{-1 / 2}$. Indeed, this is due to the following fact: If $T>1$ is given, then $\bigcup_{(C, m) \in \vartheta} e_{C}$ embeds the $|s| \leq T$ portion of any sufficiently small $\delta$ version of $\bigcup_{(C, m) \in \vartheta} N_{\delta}$. Now fix an end $\mathcal{E} \subset C$. Given $n$, use $\mathcal{E}_{n} \subset \mathcal{E}$ to denote the set of points where $z$ is the only point of $\left.N_{1}\right|_{z}$ with distance $16 \delta_{n} r_{n}^{-1 / 2}$ or less whose image via $e_{C}$ lies in $\cup_{\left(C^{\prime}, m^{\prime}\right) \in \vartheta} C^{\prime}$. It follows from $[9,(4-2)]$ that $\mathcal{E}_{n}$ is path connected and contains $|s|=T$ part of $\mathcal{E}$. This implies that $\operatorname{dist}\left(z, \alpha_{n}^{-1}(0)\right)<\delta_{n} r_{n}^{-1 / 2}$ also.

To continue, fix $\gamma \in \Xi_{\Sigma_{-}}$and reintroduce the notation used in [9, Section 5.c]. The simplest case to consider is that where $U_{\gamma-}$ contains just a single end, $\mathcal{E}$, from $\bigcup_{(C, m) \in \vartheta} C$. If this end comes from a pair $(C, 1)$, then what is said above implies that every point $z \in \mathcal{E}$ has distance $\delta_{n} r_{n}^{-1 / 2}$ or less from $\alpha_{n}^{-1}(0)$ when $n$ is large. Suppose that this end comes from $(C, m)$ with $m>1$, or that $U_{\gamma-}$ contains a second end. To treat this case reintroduce from $\left[9\right.$, Section 5.c] the partition $\left\{\mathcal{E}_{1}, \ldots, \mathcal{E}_{N}\right\}$ of the ends of $\bigcup_{(C, m) \in \vartheta} C$ that lie in $U_{\gamma-}$. The simplest case now to consider is that where there is but one partition subset, $\mathcal{E}_{1}$. In fact, this case gives the crux of the argument that works in general. To start the story for the $N=1$ case, let $\lambda<0$ denote the eigenvalue $\lambda_{q_{\mathcal{E}}}$ that appears in each $\mathcal{E} \in \mathcal{E}_{1}$ version of [9, (4-2)]. Note that there exists $c_{0} \geq 1$ with the following properties:

- If $\mathcal{E} \subset \mathcal{E}_{1}$, then the $w>\lambda^{-1} \ln \left(\delta_{n} r_{n}^{-1 / 2}\right)+c_{0}$ part of $\mathcal{E}$ contains $\mathcal{E}_{n}$.

- If $\mathcal{E} \subset \mathcal{E}_{1}$ and $z \in \mathcal{E}$ has $w(z) \leq \lambda^{-1} \ln \left(\delta_{n} r_{n}^{-1 / 2}\right)+c_{0}$, then all points in the constant $w=w(z)$ slice $U_{\gamma-} \cap\left(\bigcup_{(C, m) \in \vartheta} C\right)$ have distance at most $c_{0} 16 \delta_{n} r_{n}^{-1 / 2}$ or less from $z$.

Granted this, it follows that all points in $U_{\gamma^{-}} \cap\left(\bigcup_{(C, m) \in \vartheta} C\right)$ have distance no greater than $c_{0} \delta_{n} r_{n}^{-1 / 2}$ from $\alpha_{n}^{-1}(0)$.

In the case when there is more than one partition subset, the argument just given proves that all points in $\bigcup_{\mathcal{E} \in \mathcal{E}_{N}} \mathcal{E}$ have distance no greater than $c_{0} \delta_{n} r_{n}^{-1 / 2}$ from $\alpha_{n}^{-1}(0)$. Granted that the desired conclusion holds for the ends in $\mathcal{E}_{N}$, this argument can now 
be reapplied with only notational changes to prove that the desired conclusion holds also for the ends in $\mathcal{E}_{N-1}$. It then works for the ends in $\mathcal{E}_{N-2}$, and so on.

\section{7.b Integrals of the curvature}

This subsection begins the proof proper of Lemma 6.2. The starting point is a sequence $\left\{r_{n},\left(A_{n}, \psi_{n}\right)\right\}_{n=1,2, \ldots}$ for which $\left\{\delta_{n}\right\}_{n=1,2, \ldots}$ does not converge to zero. Thus, there exists $\delta_{0}>0$ such that $\delta_{n}>\delta_{0}$ for all $n$. The constant $c_{0}$ that appear below henceforth depends implicitly on $\min \left(1, \delta_{0}\right)$.

The substantive part of the proof is the upcoming Lemma 7.1 which concerns certain integrals of the curvature 2-form. To set the stage for the lemma, suppose that $C$ comes either from a pair in $\vartheta$, or that $C=\mathbb{R} \times \gamma$ with $\gamma$ in either $\Xi_{\Sigma_{-}}$or $\Xi_{\Sigma_{+}}$.

Recall that $N_{1} \subset N$ is a subbundle of radius $c_{0}^{-1}$ whose fibers disks are embedded by the exponential map $e_{C}$ from $[9$, Section 4.a]. Let $D \subset \mathbb{C}$ for the moment denote a given disk radius $c_{0}^{-1}$, then $e_{C}$ will embed $\left.N_{1}\right|_{D}$. Let $D^{\prime} \subset D$ denote the concentric disk with half $D$ 's radius and use $e_{C}$ to identify $\left.N_{1}\right|_{D^{\prime}}$ with its image in $\mathbb{R} \times M$. Granted this identification, then there exists an orthogonal basis for $T^{1,0}(\mathbb{R} \times M)$ of the following sort: Use $\mathfrak{s}$ to denote the tautological section over $N$ of $\pi^{*} N$ and let $\theta$ denote the Hermitian connection pulled up to $\pi^{*} N$. The basis is denoted by $\left\{\kappa_{0}, \zeta \kappa_{1}\right\}$ where $\kappa_{0}$ is a section of $\pi^{*} T^{*} C$ with norm $\sqrt{2}$ that restricts to $C$ as a section of $T^{1,0} C$. Meanwhile, $\kappa_{1}=\nabla_{\theta} \mathfrak{s}+\varsigma$ where $\varsigma$ vanishes along $\mathbb{C}$, and to order $|\mathfrak{s}|^{2}$, it is a section of $\pi^{*} T C$. In addition $|\zeta|$ differs from 1 by at most $c_{0}|\mathfrak{s}|^{2}$ and $\left|\nabla_{\theta} \zeta\right| \leq c_{0}|\mathfrak{s}|$. This constant is chosen so as to give $\kappa_{1}$ norm $\sqrt{2}$ also. Finally, the pullback of $d s \wedge a+\frac{1}{2} d a$ to each fiber disk in $\left.N_{1}\right|_{D^{\prime}}$ differs from that of $(i / 2) \kappa_{1} \wedge \bar{\kappa}_{1}$ by no more than $c_{0}|\mathfrak{s}|^{2}$.

Fix $n$ large and use $(A, \alpha)$ to denote $\left(A_{n}, \psi_{n}\right)$. Define the connection $\hat{A}$ as in (3-33) using an increasing function $x \rightarrow \wp(x)$ with $\wp=x$ near $x=0$ and with $\wp(1)=1$. Fix a disk $D \subset \mathbb{C}$ as above, write the curvature of $F_{\hat{A}}$ on $\left.N_{1}\right|_{D^{\prime}}$ using the basis $\left\{\kappa_{0}, \zeta \kappa_{1}\right\}$ as

$$
F_{\hat{A}}=f_{0} \kappa_{0} \wedge \bar{\kappa}_{0}+f_{1} \kappa_{1} \wedge \bar{\kappa}_{1}+f_{+} \kappa_{0} \wedge \kappa_{1}-\bar{f}_{+} \bar{\kappa}_{0} \wedge \bar{\kappa}_{1}+f_{-} \kappa_{0} \wedge \bar{\kappa}_{1}-\bar{f}_{-} \bar{\kappa}_{0} \wedge \kappa_{1}
$$

Lemmas 3.1, 3.6, 3.8 and 3.9 with (3-35) put constraints on the coefficients that appear here as they require that

$$
\text { - }|x|^{2} f_{1}+|y|^{2} f_{0}+\bar{x} y f_{-}+x \bar{y} \overline{f_{-}} \geq-c_{0}\left(r_{n}^{-1}+e^{-\sqrt{r} \operatorname{dist}\left(X_{*}, \cdot\right) / c_{0}}\right) \text { for any }
$$
unit vector $(x, y) \in \mathbb{C}^{2}$.

$$
\text { - }\left|f_{+}\right| \leq c_{0} r_{n}^{1 / 2}\left(r_{n}^{-1}+e^{-\sqrt{r} \operatorname{dist}\left(X_{*}, \cdot\right) / c_{0}}\right) \text {. }
$$

Given $p \in C$ and $\rho>0$, use $D_{\rho}$ in what follows to denote the disk of radius $\rho$ centered at $p$. Assume in all cases that $\rho$ is such that the exponential map $e_{C}$ embeds $N_{1} \mid D_{4 \rho}$ 
Now suppose that $\mathfrak{z}>0$ is less than the radius of the fiber disks in $N_{1}$. (Such an upper bound is also assumed implicitly below.) Use $N_{z} \subset N_{1}$ in what follows to denote the radius $z$ subbundle. With $z$ given, introduce $\chi_{z}$ to denote the function $\chi(|\mathfrak{s}| / z)$ on $N_{1}$. Define the set $X_{*} \subset \mathbb{R} \times M$ to be the region where $|\alpha| \leq 1-\kappa^{-1}$, with $\kappa$ as given by Lemma 3.8.

Lemma 7.1 Given $c>0$ and $k \in\{0,1, \ldots\}$, there exists a constant $\kappa>1$ with the following significance: Fix $C$ and $p \in C$. Take $n \geq \kappa$ and fix $\rho \in\left(\delta_{n} r_{n}^{-1 / 2}, \kappa^{-2}\right)$, then $z \in\left(\kappa \rho, \kappa^{-1}\right)$, and then $\rho_{\diamond} \in\left[8 \delta_{n} r_{n}^{-1 / 2}, z\right]$. Assume that $\left.\alpha^{-1}(0) \cap N\right|_{D_{2 \rho}}$ does not contain points with $|\mathfrak{s}| \in\left[\frac{1}{8} \rho \diamond, z\right]$. In what follows, $\Delta=0$ if $\wp\left(|\alpha|^{2}\right)=1$ at all points with $|\mathfrak{s}| \geq \frac{1}{4} z$; otherwise $\Delta=1$.

$$
\begin{aligned}
& \text { - } \int_{\pi^{-1}\left(D_{\rho}\right)} \chi_{z}|\mathfrak{s}|^{k} e^{-\sqrt{r_{n}} \operatorname{dist}\left(X_{*}, \cdot\right) / c} \leq \kappa \rho^{2}\left(\rho_{\diamond}\right)^{k} r_{n}^{-1} . \\
& \text { - } \int_{\pi^{-1}\left(D_{\rho}\right)} \chi_{z}|\mathfrak{s}|^{k}\left|F_{\hat{A}}\right| \leq \kappa \rho^{2} \rho_{\diamond}^{k}\left(1+\Delta\left(z / \rho_{\diamond}\right)^{k} r_{n}^{-1}\right) \\
& \cdot \int_{\pi^{-1}\left(D_{\rho}\right)} \chi_{z}|\mathfrak{s}|^{k}\left|f_{+}\right| \leq \kappa \rho^{2} r_{n}^{-1 / 2} \rho_{\diamond}^{k}\left(1+\Delta\left(z / \rho_{\diamond}\right)^{k} r_{n}^{-1}\right) . \\
& \text { - } \int_{\pi^{-1}\left(D_{\rho}\right)} \chi_{z}|\mathfrak{s}|^{k}\left(\left|f_{0}\right|+\left(\frac{\rho_{\diamond}}{\rho}\right)^{1 / 2}\left|f_{-}\right|\right) \\
& \quad \leq \kappa \rho^{2} \rho_{\diamond}^{k}\left(\rho_{\diamond} / \rho+\Delta e^{-\sqrt{r_{n}} z / \kappa}\right)\left(1+\Delta\left(z / \rho_{\diamond}\right)^{k} r_{n}^{-1}\right) .
\end{aligned}
$$

The following is a parenthetical remark with regards to the $\Delta=0$ case: Lemma 3.7 and Lemma 3.8 say that given $\delta>0$, there exists an $n$-independent constant $\kappa_{\delta}$ such that $|\alpha| \geq 1-\delta$ where $|\mathfrak{s}| \geq\left(\frac{1}{8} \delta_{n}+\kappa_{\delta}\right) r_{n}^{-1 / 2}$. This understood, there will be choices for $\wp$ and $z$ for which the condition on $\wp\left(|\alpha|^{2}\right)$ follows from the earlier constraint that $|\mathfrak{s}| \leq \frac{1}{8} \rho_{\diamond}$ on the zero locus of $\alpha$.

As noted in the introduction to this section, there is a gap in the proof of Lemma 5.5 from the article $\mathrm{SW}=\mathrm{Gr}$ in [6]. The argument that follows proving Lemma $7.1 \mathrm{can}$ be used to prove the following: In the context of what is assumed for Lemma 5.5 in the article $\mathrm{SW}=\mathrm{Gr}$ from [6], the assertions of Lemma 7.1 hold with $\Delta=0$ and $\wp(x)=x$. The latter result can be used in lieu of Lemma 5.5 for the parts in this $\mathrm{SW}=\mathrm{Gr}$ article that refer to its Lemma 5.5.

Proof of Lemma 7.1 The proof has two steps.

Step 1 This step proves the first item. To start, fix $\chi \in\left(r^{-1 / 2}, z\right)$ and then fix a maximal set, $\Lambda_{\chi}$, of points in the portion of $\alpha^{-1}(0)$ that lies in $\left.N_{z / 2}\right|_{D_{\rho}}$ with the 
following property: Any two distinct points from $\Lambda_{\chi}$ have distance at least $\chi$ apart. According to Lemma 3.10, the integral of $r\left(1-|\alpha|^{2}\right)$ over the ball of radius $4 \chi$ centered at any given point in $\Lambda_{\chi}$ can be no greater than $c_{0} \chi^{2}$. Note that $\left.\alpha^{-1}(0) \cap N_{z / 2}\right|_{D_{\rho}}$ is covered by the union of these balls of radius $4 \chi$ and center in $\Lambda_{\chi}$. If $\chi<c_{0} \rho$, then this set can have at most $c_{0} \rho^{2} \chi^{-2}$ elements when $n$ is large if $z \leq c_{0}^{-1}$. This follows from Lemma 6.1. To elaborate, note that if $z \leq c_{0}^{-1}$, then $\bigcup_{\left(C^{\prime}, m^{\prime}\right) \in \vartheta} C^{\prime}$ intersects $\left.N_{z / 2}\right|_{D_{2 \rho}}$ only in $D_{2 \rho}$ unless $D_{2 \rho}$ is far out on an end of $\bigcup_{\left(C^{\prime}, m^{\prime}\right) \in \vartheta} C^{\prime}$. In the latter case, each component of the intersection is the image of a nearly parallel section over $D_{2 \rho}$ of $N_{z}$. By the same token, if $\chi>\rho$, then there are at most $c_{0}$ elements in $\Lambda_{\chi}$. Now let $U_{\chi}$ denote the union of the intersections of the balls of radius $\chi$ centered at the points of $\Lambda_{\chi}$ with $\left.N_{z}\right|_{D_{\rho}}$.

Consider first the case when $k=0$. The integral is no greater than the sum, indexed by the integers $q \in\left[1, c_{0} r^{1 / 2} z\right]$, of the contributions from $U_{q / \sqrt{r}}-U_{(q-1) / \sqrt{r}}$. Here, $U_{0}=$ $\varnothing$. To bound this sum, remark that the factor $e^{-\sqrt{r} \operatorname{dist}\left(X_{*}, \cdot\right) / c}$ is less than $e^{-\sqrt{r} x / c c_{0}}$ on $\left.N_{z}\right|_{D_{\rho}}-U_{\chi}$. Granted this, any given radius $q r^{-1 / 2}$ ball with center in $\Lambda_{q / \sqrt{r}}$ contributes at most $e^{-q / c c_{0}}$ times the volume of its intersection with $\left.N_{z}\right|_{D_{\rho}}$. Given what was said about the maximum number of such balls, it follows that the left hand side of (7-3) can never be greater than $c_{0} \sum_{q=0,1,2, \ldots} e^{-q / c c_{0}}\left(\rho^{2} /\left(q r^{-1 / 2}\right)^{2}\left(q r^{-1 / 2}\right)^{4}\right.$, which is less than $c_{0} \rho^{2}-c r^{-1}$.

Consider next the case when $k>0$. If $q / \sqrt{r}<16 \rho_{\diamond}$, then the contribution from a ball with center in $\Lambda_{q / \sqrt{r}}$ is at most $c_{0} \rho_{\diamond}^{k} e^{-q / c c_{0}}$ times the volume of its intersection with $\left.N_{z}\right|_{D_{\rho}}$. If $q / \sqrt{r} \geq 16 \rho_{\diamond}$, then the contribution is at most $c_{0}(q / \sqrt{r})^{k} e^{-q / c c_{0}}$ times the volume of its intersection with $\left.N_{z}\right|_{D_{\rho}}$. These contributions sum to less than $c_{0}\left(\rho_{\diamond}^{k}+r^{-k / 2}\right) \rho^{2} r^{-1}$.

Step 2 What with (3-35) and (7-4), the second and third items of the lemma follow directly from the first item of the lemma.

To prove the fourth item, note first that $\chi_{z}=1$ on the support of $F_{\hat{A}}$ if $\wp\left(|\alpha|^{2}\right)=1$ where $|\mathfrak{s}| \geq \frac{1}{4} z$. Now, let $\chi^{D}$ denote the function on $C$ that assigns to $u \in C$ the value of $\chi\left(4 \operatorname{dist}_{C}(p, u) / \rho\right)$. Here, $\operatorname{dist}_{C}(\cdot, \cdot)$ denotes the distance as measured on $C$. Use pullback via the map $\pi$ to view $\chi^{D}$ as a function on $\pi^{-1}(C)$. The integral of $\chi_{z}\left|f_{0}\right|$ over $\pi^{-1}\left(D_{\rho}\right)$ is less than that of $\chi^{D} \chi_{z}\left|f_{0}\right|$ over $\pi^{-1}\left(D_{2 \rho}\right)$. To bound the latter integral, note that the $x=0$ version of (7-4) finds $f_{0} \geq\left|f_{0}\right|-$ $c_{0}\left(r_{n}^{-1}+e^{-\sqrt{r_{n}} \operatorname{dist}\left(X_{*}, \cdot\right) / c_{0}}\right)$. This understood, it follows from the first item of the lemma that up to an error that is at most $c_{0} \rho^{2} \rho_{\diamond}^{k}\left(1+\Delta\left(z / \rho_{\diamond}\right)^{k}\right) r_{n}^{-1}$, the integral over $\pi^{-1}\left(D_{2 \rho}\right)$ of $\chi^{D}|\mathfrak{s}|^{k}\left|f_{0}\right|$ is no greater than the integral of $\chi^{D} f_{0}$ over $\pi^{-1}\left(D_{2 \rho}\right)$. 
This is, up to a factor,

$$
\int_{\pi^{-1}\left(D_{2 \rho^{\prime}}\right)} \chi^{D} \chi_{z}|\mathfrak{s}|^{k}\left(F_{\hat{A}} \wedge \kappa_{1} \wedge \bar{\kappa}_{1}\right)
$$

To bound (7-5), note that $\kappa_{1} \wedge \bar{\kappa}_{1}$ can be written as $\operatorname{im}\left(d\left(\zeta \mathfrak{s}^{k+1} \bar{\kappa}_{1}\right)\right)+\mathfrak{o}$, where $|\mathfrak{o}| \leq$ $c_{0}|\mathfrak{s}|$ and where $\operatorname{im}(\cdot)$ designates the imaginary part of the indicated $\mathbb{C}$-valued form. It follows from (3-34), Lemma 3.8, and the second item of the lemma that the absolute value of the contribution of $\mathfrak{o}$ to (7-5) is less than $c_{0} \rho^{2} \rho_{\diamond}^{k+1}\left(1+\Delta\left(z / \rho_{\diamond}\right)^{k+1} r_{n}^{-1}\right)$. To analyze the $\operatorname{im}\left(d\left(\zeta \mathfrak{s}^{k+1} \bar{\kappa}_{1}\right)\right)$ contribution to the integral in (7-5), integrate by parts to see that this contribution is no greater than

$$
c_{0}\left|\int_{\pi^{-1}\left(D_{2 \rho}\right)}\left(d \chi^{D} \chi_{z}+\chi^{D} d \chi_{z}\right) \wedge F_{\hat{A}} \wedge \operatorname{im}\left(\zeta_{\mathfrak{s}}^{k+1} \bar{\kappa}_{1}\right)\right| .
$$

The contribution of the part of the integrand in (7-6) with $d \chi_{z}$ is no greater than

$$
c_{0} \rho^{2} \rho_{\diamond}^{k} \Delta\left(e^{-\sqrt{r_{n}} z / \kappa}+\left(z / \rho_{\diamond}\right)^{k} r_{n}^{-1}\right) .
$$

Indeed, this follows from (3-35), Lemma 3.8 and the second item of Lemma 7.1. Meanwhile, the contribution to (7-6) from $d \chi^{D}$ is no greater than

$$
\left.c_{0} \rho^{-1} \int_{\pi^{-1}\left(D_{2 \rho}\right)} \chi\right|_{z}|\mathfrak{s}|^{k+1}\left(\left|f_{-}\right|+\left|f_{+}\right|\right) .
$$

It follows from the second item in the lemma that what is written here is no greater than $c_{0} \rho^{2}\left(\rho_{\diamond} \rho^{-1}\right) \rho_{\diamond}^{k}\left(1+\Delta\left(z / \rho_{\diamond}\right)^{k} r_{n}^{-1}\right)$.

What was said in the preceding paragraphs directly implies the asserted bound for $\left|f_{0}\right|$ 's contribution to the integral in the fourth item of the lemma. To derive the bound asserted for the $\left|f_{-}\right|$contribution, use the first item in (7-4) with $x=-(\rho \diamond / \rho)^{1 / 2} f_{-} /\left|f_{-}\right|$and with $y=1$ to bound $(\rho \diamond / \rho)^{1 / 2}\left|f_{-}\right|$by

$$
c_{0}\left(\left|f_{0}\right|+\left(\rho_{\diamond} / \rho\right)\left|f_{1}\right|+r_{n}^{-1}+e^{-\sqrt{r_{n}} \operatorname{dist}\left(X_{*}, \cdot\right) / c_{0}}\right) .
$$

\section{7.c Special sections of $N$}

To set the stage for the lemma, suppose that $C$ comes either from a pair in $\vartheta$, or that $C=\mathbb{R} \times \gamma$ with $\gamma$ in either $\Xi_{\Sigma-}$ or $\Xi_{\Sigma+}$. The purpose of this subsection is to construct some special sections of powers of the normal bundle over parts of $C$.

To set the notation, first introduce $\omega_{C}$ to denote the induced volume form on $C$. The upcoming definition also requires a choice of $\rho \in\left(0, c_{0}^{-1}\right)$. With $\rho$ given, each $p \in C$ is assigned the function $\chi^{p}(\cdot)=\chi(\operatorname{dist}(p, \cdot) / \rho)$. The definition also requires the specification of numbers $z>0$ and $\rho_{\diamond}>0$. 
Fix an increasing function $\wp:[0, \infty) \rightarrow[0, \infty)$ such that $\wp(x)=x$ near 0 and such that $\wp(1)=1$. In what follows, it is sufficient to consider only two versions of $\wp$, the first being $\wp_{1}(x)=x$ and the second $\wp_{2}(x)=x \chi(x)+1-\chi(x)$. Given an index $n$, use $\hat{A}$ to denote the $\left(A_{n}, \psi_{n}\right)$ version of (3-33) as defined using the function $\wp$. Note that $\hat{A}$ is flat where $|\alpha|^{2} \geq \frac{1}{2}$ when $\wp=\wp_{2}$.

Assign to each integer $k \geq 0$ the section, $\mathfrak{o}_{k}=\mathfrak{o}_{(n) k}$ of $N^{\otimes k}$ using the rule

$$
\left.\mathfrak{o}_{k}\right|_{p}=\rho^{-2} \rho_{\diamond}^{-k} \int_{N_{z}} \chi^{p} \chi_{z^{\mathfrak{s}^{k}}}\left(\frac{i}{2 \pi} F_{\hat{A}}\right) \wedge \pi^{*} \omega_{C} .
$$

Note that the appearance of the function $\chi_{z}$ in (7-9) is superfluous in the case $\wp=\wp_{2}$ if $|\alpha|^{2} \geq \frac{1}{2}$ at the points where $|\mathfrak{s}| \geq \frac{1}{4} z$. This is because $F_{\hat{A}}=0$ where $\chi_{z} \leq 1$.

The next lemma says the first things about $\mathfrak{o}_{k}$. The statement of this lemma refers to the coefficients $\left(v_{C}, \mu_{C}\right)$ that appear in [9, (4-5)]. It also refers to an integer $m$. When $C$ comes from $\vartheta$, the latter is the integer that pairs with $C$. If $C=\mathbb{R} \times \gamma$ with $\gamma \in \Xi_{\Sigma-}$ or with $\gamma \in \Xi_{\Sigma+}$, then $m=m_{\gamma-}$ or $m=m_{\gamma+}$, respectively. Note $\mu_{C}=0$ in all cases when $m>1$.

Lemma 7.2 Given a positive integer $k$, there exists a constant $\kappa>1$ with the following significance: Suppose that $n \geq \kappa$. Fix $\rho \in\left[\delta_{n} r_{n}^{-1 / 2}, \kappa^{-2}\right), z \geq \kappa \rho$, and then $\rho_{\diamond} \in\left[8 \delta_{n} r_{n}^{-1 / 2}, z\right]$. Suppose that $D_{\rho} \subset C$ is an embedded disk such that $\left.N_{z}\right|_{D_{2 \rho}}$ contains no points in $\alpha^{-1}(0)$ with $|\mathfrak{s}| \in\left[\frac{1}{8} \rho_{\diamond}, z\right]$. Define $\mathfrak{o}_{k}=\mathfrak{o}_{(n) k}$ at points in $D_{\rho}$ using the formula in (7-9).

$$
\begin{aligned}
\left|\mathfrak{o}_{k}\right| & \leq \kappa\left(1+\left(z / \rho_{\diamond}\right)^{k} r_{n}^{-1}\right) \\
\left|\bar{\partial}_{\theta} \mathfrak{o}_{k}\right| & \leq \kappa\left(1+\left(z / \rho_{\diamond}\right)^{k}\left(r_{n}^{-1}+z^{-1} e^{-\sqrt{r_{n}} z / \kappa}\right)\right) .
\end{aligned}
$$

Moreover,

- In the case $k=1$, write $\bar{\partial}_{\theta} \mathfrak{o}_{1}+v_{C} \mathfrak{o}_{1}+\mu_{C} \overline{\mathfrak{o}}_{1}=\mathfrak{e}$.

(a) If $\rho_{\diamond}<\rho$, then

$$
|\mathfrak{e}| \leq \kappa\left(\left(\rho_{\diamond} / \rho\right)^{1 / 2}+\left(\rho / \rho_{\diamond}\right)^{1 / 2} z^{-1} e^{-\sqrt{r_{n}} z / \kappa}+\left(\rho_{\diamond}\right)^{-1} r_{n}^{1 / 2}\right)\left(1+\left(z / \rho_{\diamond}\right)^{2} r_{n}^{-1}\right) .
$$

(b) If $D_{2 \rho}$ lies in an end of $C$ and if $\hat{A}$ is defined using $\wp(\cdot)$ with $\wp\left(|\alpha|^{2}\right)=1$ where

$|\mathfrak{s}| \geq \frac{1}{4} z$ on $N_{z} \mid D_{2 \rho}$, then $\left|\mathfrak{o}_{1}\right| \leq \kappa$ and $|\mathfrak{e}| \leq \kappa\left(\rho+\left(\rho_{\diamond} / \rho\right)^{1 / 2}+\left(\rho_{\diamond}\right)^{-1} r_{n}^{-1}\right)$.

- Suppose that $m>1$ and that $\hat{A}$ using $\wp(\cdot)$ with $\wp\left(|\alpha|^{2}\right)=1$ where $|\mathfrak{s}| \geq \frac{1}{4} z$ on $\left.N_{z}\right|_{D_{2 \rho}}$. Take $k \in\{1, \ldots, m\}$. Then $\left|\mathfrak{o}_{k}\right| \leq \kappa$ and $\left|\bar{\partial}_{\theta} \mathfrak{o}_{k}+k v_{C} \mathfrak{o}_{k}\right| \leq$ $\kappa\left(\rho_{\diamond}^{2}+\left(\rho_{\diamond}\right)^{-1} r_{n}^{-1}\right)$. 


\section{Proof of Lemma 7.2 The proof has four steps.}

Step 1 The fact that $\left|\mathfrak{o}_{k}\right| \leq c_{0}\left(1+\left(z / \rho_{\diamond}\right)^{k} r_{n}^{-1}\right)$ follows from Lemma 7.1. To see about the asserted bound for $\left|\bar{\partial}_{\theta} \mathfrak{o}_{k}\right|$, use parallel transport by $\theta$ from the center point of $D_{\rho}$ to define a product structure for $N$ over the disk $D_{2 \rho}$. Writing $N$ here as $D_{2 \rho} \times \mathbb{C}$ identifies the section $\mathfrak{s}$ of $\pi^{*} N \rightarrow N$ with the complex coordinate $z \in \mathbb{C}$. This done, then $\mathfrak{o}_{k}$ can be viewed as the $\mathbb{C}$-valued function:

$$
\left.\mathfrak{o}_{k}\right|_{p}=\frac{i}{2 \pi} \rho^{-2} \rho_{\diamond}^{-k} \int_{\mathbb{C} \times D_{2 \rho}} \chi^{p} \chi_{z} \mathfrak{f}_{1} z^{k} d z \wedge d \bar{z} \wedge \omega_{C},
$$

where $\mathfrak{f}_{1}$ is the $d z \wedge d \bar{z}$ component of $F_{\hat{A}}$. Note that if $\wp(x)=x \chi(x)+1-\chi(x)$, then the assumption $|\alpha|^{2}>\frac{1}{2}$ at the points where $|\mathfrak{s}| \geq \frac{1}{4} z$ implies that the function $\chi_{z}$ in (7-9) is 1 on the support of $\left|F_{\hat{A}}\right|$.

Now fix a complex, Gaussian coordinate for $C$ centered at $p$; this a complex function $u$ such that $\left.u\right|_{p}=0$, and $d u$ spans $T^{1,0} C$ near $p$ with $|d u|=\sqrt{2}$ at $p$. Granted (7-10), act on $\mathfrak{o}_{k}$ by $\partial / \partial \bar{u}$. The result, after an integration by parts and an appeal to Lemma 7.1 can be seen to differ by at most $c_{0} \rho\left(1+\left(z / \rho_{\diamond}\right)^{k} r_{n}^{-1}\right)$ from

$$
-\frac{1}{4 \pi} \rho^{-2} \rho_{\diamond}^{-k} \int_{\mathbb{C} \times D_{2 \rho}} \chi^{p} \chi_{z}\left(\frac{\partial}{\partial \bar{u}} \mathfrak{f}_{1}\right)(1+g) z^{k} d z \wedge d \bar{z} \wedge d u \wedge d \bar{u} .
$$

Here, $g=g(u)$ is defined by writing $\omega_{C}=\frac{i}{2}(1+g) d u \wedge d \bar{u}$. To proceed from here, use the fact that $F_{\hat{A}}$ is closed to write

$$
\frac{\partial}{\partial \bar{u}} \mathfrak{f}_{1}=+\frac{\partial}{\partial \bar{z}} \overline{\mathfrak{f}}_{-}-\frac{\partial}{\partial z} \overline{\mathfrak{f}}_{+},
$$

where $\mathfrak{f}_{+}$is the $d u \wedge d z$ component of $F_{\hat{A}}$ and $\mathfrak{f}_{-}$is the $d u \wedge d \bar{z}$ component. Insert this into (7-11) and integrate by parts again. It follows from (3-34) and Lemma 7.1 that the contribution from $\overline{\mathfrak{f}}_{-}$to (7-11) is no greater than $c_{0} z^{-1} e^{-\sqrt{r} z / \kappa}(z / \rho \diamond)^{k}$. However, in the case where $m>1$ or $C=\mathbb{R} \times \gamma$ with $\gamma$ an elliptic Reeb orbit, the contribution from $\overline{\mathfrak{f}}_{-}$to $(7-11)$ is zero as can be seen after integrating by parts. Meanwhile, an integration by parts finds that the contribution from $\overline{\mathfrak{f}}_{+}$is

$$
-\frac{1}{4 \pi} k \rho^{-2} \rho_{\diamond}^{-k} \int_{C \times D_{2 \rho}} \chi^{p} \chi_{z} \overline{\mathfrak{f}}_{+}(1+g) z^{k-1} d z \wedge d \bar{z} \wedge d u \wedge d \bar{u} .
$$

To finish the story, note that $\mathfrak{f}_{+}$can be written in terms of the coefficients in (7-3) as

$$
\mathfrak{f}_{+}=f_{+}+g_{1} f_{1}+g_{-+}+f_{-}+g_{--} \bar{f}_{-}+g_{0} f_{0}+\widehat{g}\left(F_{\hat{A}}\right),
$$

where $|\hat{g}| \leq c_{0}|z|^{2}$ and $\left|g_{1}\right|+\left|g_{-+}\right|+\left|g_{--}\right|+\left|g_{0}\right| \leq c_{0}|z|$. This being the case, the claimed bound for $\left|\bar{\partial}_{\theta} \mathfrak{o}_{k}\right|$ follows from (7-14) using the second and third items Lemma 7.1. 
Step 2 To obtain Assertion (a) of the first bullet of the lemma, substitute the left hand side of (7-14) for $\mathfrak{f}_{+}$in (7-13). What with the first item of Lemma 7.1, the argument that leads from Equation (5.32) in the article SW = Gr from [6] to Equation (5.33) in this same article can be invoked to see that the $g_{1} f_{1}$ term gives the $v_{C}$ and $\mu_{C}$ terms with an error of size at most $c_{0} \rho \diamond\left(1+\left(z / \rho_{\diamond}\right)^{2} r_{n}^{-1}\right)$. Use the third item of Lemma 7.1 to bound the $f_{+}$contribution by $c_{0}\left(\rho \diamond r^{1 / 2}\right)^{-1}\left(1+(z / \rho \diamond) r_{n}^{-1}\right)$. Use the fourth item of Lemma 7.1 to bound the $f_{-}$and $f_{0}$ contributions by $c_{0}\left((\rho \diamond / \rho)^{1 / 2}+\right.$ $\left.(\rho / \rho \diamond)^{1 / 2} e^{-\sqrt{r} z / \kappa}\right)\left(1+(z / \rho \diamond) r_{n}^{-1}\right)$.

Step 3 To obtain Assertion (b) of the first bullet, note first that the bound on $\left|\mathfrak{o}_{1}\right|$ comes directly from Lemma 7.1. To bound $|\mathfrak{e}|$, note that the function $\chi_{z}$ that appears in (7-10) is equal to 1 on the support of $F_{\hat{A}}$. This understood, it follows using Lemma 7.1 that $(\partial / \partial \bar{u}) \mathfrak{o}_{1}$ differs from (7-11) by no more than $c_{0} \rho$. Substitute (7-12) into (7-11). Because $\chi_{z}=1$, there is no contribution from the $\overline{\mathfrak{f}}_{-}$term. Furthermore integration by parts finds (7-11) to be equal on the nose to (7-13). Use (7-14) with Lemma 7.1 to see that the $f_{1}$ term contributes the $v_{C}$ and $\mu_{C}$ terms to the expression for $\mathfrak{e}$ plus an error that is no greater than $c_{0} \rho \diamond$. Meanwhile, Lemma 7.1 finds that the contribution from the $f_{-}$and $f_{0}$ terms are bounded in absolute value by $c_{0}(\rho \diamond / \rho)$. Finally, the contribution from the $f_{+}$term is no greater than $c_{0} \rho_{\diamond}^{-1} r_{n}^{-1}$. To see why, use (3-34) to see that

$$
\bar{f}_{+}=r(1-\wp) \bar{\alpha} \beta-\wp^{\prime}\left(\bar{\partial}_{A 0} \bar{\alpha} \bar{\partial}_{A 1} \alpha-\bar{\partial}_{A 1} \bar{\alpha} \bar{\partial}_{A 0} \alpha\right)
$$

where $\left\{\bar{\partial}_{A 0}, \bar{\partial}_{A 1}\right\}$ denote the respective covariant derivatives for any given connection $A$ along the vector fields that are dual to the basis $\left\{\bar{\kappa}_{0}, \bar{\kappa}_{1}\right\}$. Given that the top equation in (1-11) equates the $\bar{\partial}_{A 0}$ and $\bar{\partial}_{A 1}$ derivatives of $\alpha$ with derivatives of $\beta$, the claimed bound on the integral of $\chi^{p}\left|f_{+}\right|$follows from (7-16) using Lemma 3.11 and the first item of Lemma 7.1 .

Step 4 This step derives the refined bounds for the case $m>1$ that is asserted by the lemma's second bullet. The bound on $\left|\mathfrak{o}_{k}\right|$ follows directly from Lemma 7.1. To bound $|\mathfrak{e}|$, first introduce $u_{p}=w_{p}+i t_{p}$ to denote the coordinates of the point $p \in \mathbb{R} \times \gamma$ as written using the coordinates from [9, Property 5 in Section 4.a]. The function $\chi^{p}(\cdot)$ in (7-10) is equal to $\chi\left(\left|u_{p}-(\cdot)\right| / \rho\right)$. The form $\omega_{C}$ in (7-10) is $(i / 2) d u \wedge d \bar{u}$. As a consequence, the function $g$ that appears in (7-11) is zero and the $g=0$ version of (7-11) is exactly $\bar{\partial} \mathfrak{o}_{k}$. Meanwhile, $\chi_{z}$ is equal to 1 on the support of $F \hat{A}$. This, understood, use (7-12) and then integrate by parts to see that $\bar{\partial} \mathfrak{o}_{k}$ is given by (7-13) with $g=0$ and $\chi_{z}=1$. 
To continue, remark that $\kappa_{0}$ in this case is equal to $\kappa_{0}=\left(\ell_{\gamma} /(2 \pi)\right)\left(1+|z|^{2}\right)^{-1 / 2} d u$. Meanwhile,

(7-16) $\kappa_{1}=\frac{\ell_{\gamma}}{2 \pi}\left(1+|z|^{2}\right)^{1 / 2}\left(1+2|z|^{2}\right)^{-1 / 2}\left(d z-\frac{1}{2} \mathrm{R} z(d u-d \bar{u})+z\left(1+|z|^{2}\right)^{-1} d u\right)$.

As a consequence, the term that is denoted by $g_{1}$ in (7-14) contributes the factor $k v_{C}=\frac{1}{2} k \mathrm{R}$ to the left hand side of what is written in the second bullet of the lemma with an error that is no greater than $c_{0} \rho_{\diamond}^{2}$. Meanwhile, both $g_{--}$and $g_{-+}$are zero in (7-14). Furthermore, what is written as $\widehat{g}$ can also be taken to be zero. This understood, it only remains to account for the $f_{+}$contribution to $e$. The latter is no greater than $c_{0} \rho_{\diamond}^{-1} r^{-1}$. This follows using [8, (3-18)], (7-15), Lemma 3.11 and the first item of Lemma 7.1.

Lemma 7.2 leads to the following corollary.

Lemma 7.3 Take $(C, m)$ as in Lemma 7.2. Given a positive integer $k$ and $v_{*} \in(0,1)$, there is a constant $\kappa>1$ with the following significance: Suppose that $I \in \mathbb{R}$ is an interval of length at least three and let $I_{1} \subset I$ denote the subset with distance at least 1 from any boundary point of $I$. Define $\mathfrak{o}_{k}$ at points in $C \cap\left(I_{1} \times M\right)$ by the formula in (7-9) using the data and under the assumptions in one of the next two bullets.

- Fix $n \geq \kappa$, fix $\rho \in\left[\delta_{n} r_{n}^{-1 / 2}, \kappa^{-2}\right)$ and $\rho \diamond \in\left[8 \delta_{n} r_{n}^{-1 / 2}, \kappa^{-2}\right]$, and set $z=$ $\max \left\{\rho_{\diamond}, r_{n}^{-1 / 2}\left(\operatorname{lnr}_{n}\right)^{2}\right\}$. Assume that $\left.N_{z}\right|_{C \cap(I \times M)}$ contains no points from $\alpha^{-1}(0)$ with $|\mathfrak{s}| \in\left[\frac{1}{8} \rho \diamond, z\right]$.

- If $C$ is accompanied by an integer $m>1$, fix $n \geq \kappa$, and fix $\rho \in\left[\delta_{n} r_{n}^{-1 / 2}, \kappa^{-2}\right)$, $\rho \diamond \in\left[8 \delta_{n} r_{n}^{-1 / 2}, \kappa^{-2}\right]$, and $z \geq \rho \diamond$. Assume that $\wp\left(|\alpha|^{2}\right)=1$ on $\left.N_{z}\right|_{C \cap(I \times M)}$ where $|\mathfrak{s}| \geq \frac{1}{4} z$.

In either case, $\mathfrak{o}_{k}$ is Holder continuous with exponent $v_{*}$ on $C \cap\left(I_{1} \times M\right)$ and it has Holder norm no greater than $\kappa$. Moreover, if $\chi \in(0,1)$ and $D_{\chi} \subset C \cap\left(I_{1} \times M\right)$ is an embedded disk of radius $\chi$, then the $L_{1}^{2}$ norm of $\mathfrak{o}_{k}$ on $D_{\chi}$ is bounded by $\kappa x(1+|\ln \chi|$.

Proof of Lemma 7.3 This follows from Lemma 7.2's bound on $\left|\mathfrak{o}_{k}\right|$ and $\left|\bar{\partial}_{\theta} \mathfrak{o}_{k}\right|$ using standard properties of the $d$-bar operator and its Green's function.

The final lemma in this subsection describes circumstances that guarantee a uniformly positive lower bound for the absolute value of at least one of Lemma 7.2's sections $\left\{\mathfrak{o}_{k}\right\}_{k=1,2, \ldots}$. To set the stage, suppose that $C$ is as described in Lemma 7.2. Given $p \in C$, suppose that a large index $n$ has been chosen; and suppose that $\rho$ and $z$ are 
as described in Lemma 7.2. Now assume that $\alpha$ has no zeros on $\left.N_{z}\right|_{D_{2 \rho}}$ at any point with $|\mathfrak{s}| \in\left[\frac{1}{4} z, z\right]$. If such is the case, then the connection

$$
A^{\prime}=A-\frac{1}{2}\left(\chi_{z}-\left(1-\chi_{z}\right)|\alpha|^{-2}\right)\left(\bar{\alpha} \nabla_{A} \alpha-\alpha \nabla_{A} \bar{\alpha}\right)
$$

is well defined on $E$ 's restriction to $\left.N_{z}\right|_{D_{2 \rho}}$. Note in particular that $A^{\prime}$ is flat and $\alpha|\alpha|^{-1}$ is $A^{\prime}$ covariantly constant where $|\mathfrak{s}| \geq \frac{3}{8} z$. As a consequence, the integral of $(i /(2 \pi)) F_{A^{\prime}}$ over any fiber disk of $\left.N_{z}\right|_{D_{2 \rho}}$ is a fixed integer. The latter is denoted by $\hat{m}$ in the upcoming lemma. Of course, if $C$ comes as the pair $(C, m) \in \vartheta$, then $\hat{m}=m$ when $D_{2 \rho}$ is the sole component of $\left.\left(\bigcup_{\left(C^{\prime}, m^{\prime}\right) \in \vartheta} C^{\prime}\right) \cap N_{z}\right|_{D_{2 \rho}}$.

Lemma 7.4 Given $\varepsilon_{*} \in(0,1)$ and $\chi \geq 1$, there is a constant $\kappa>1$ with the following significance: Suppose $(C, m)$ are as in Lemma 7.2. Take $n \geq \kappa$ and $\rho \in\left[\chi \delta_{n} r_{n}^{-1 / 2}, \kappa^{-2}\right]$. Given $p \in C$, construct $\mathfrak{o}_{k}$ on $D_{2 \rho}$ for each $k \in k \in\{1, \ldots, \hat{m}\}$ using the data and under the assumptions in one of the next two bullets.

- Take $\rho$ as above, $\rho_{\diamond} \in\left[8 \delta_{n} r_{n}^{-1 / 2}, \kappa^{-2}\right]$ and set $z=\max \left\{\rho_{\diamond}, r_{n}^{-1 / 2}\left(\operatorname{lnr}_{n}\right)^{2}\right\}$. Assume that $\left.N_{z}\right|_{C \cap(I \times M)}$ contains no points from $\alpha^{-1}(0)$ with $|\mathfrak{s}| \in\left[\frac{1}{8} \rho_{\diamond}, z\right]$.

- If $C$ is accompanied by an integer $m>1$, take $\rho$ as above, $\rho_{\diamond} \in\left[8 \delta_{n} r_{n}^{-1 / 2}, \kappa^{-2}\right]$, and then $z \geq \rho_{\diamond}$. Assume that $\wp\left(|\alpha|^{2}\right)=1$ on $\left.N_{z}\right|_{C \cap(I \times M)}$ where $|\mathfrak{s}| \geq \frac{1}{4} z$.

In either case, assume that there exists $z \in N_{z} \mid p$ with $\alpha(z)=0$ and $|\mathfrak{s}| \geq \varepsilon_{*} \rho_{\diamond}$. Then there exists $k \in\{1, \ldots, \hat{m}\}$ such that $\left|\mathfrak{o}_{k}\right|_{p} \mid \geq \kappa^{-1}$.

Proof of Lemma 7.4 The following fact is justified momentarily: With $\rho, z$ and $\rho \diamond$ given, there exists $\kappa \geq 1$ independent of $n$ such that if $n \geq \kappa$, then

$$
\rho^{-2} \int_{N_{z}} \chi^{p}\left(\frac{i}{2 \pi} F_{\hat{A}}\right) \wedge \pi^{*} \omega_{C}=n_{p} \hat{m}+\mathfrak{e},
$$

where $n_{p}$ is defined by the rule $n_{p}=\rho^{-2} \int_{\mathbb{C}} \chi^{p}$. Meanwhile, $\mathfrak{e}=0$ if the second bullet in the lemma holds, and $|\mathfrak{e}| \leq c_{0} \mathfrak{e}^{-\sqrt{r_{n}} z / c_{0}}$ otherwise. Hold on to (7-18).

To continue, use parallel transport from $p$ along the radial geodesics in $D_{2 \rho}$ to give a product structure to $\left.N\right|_{D_{2 \rho}}$. Fix $\varepsilon \in\left(0, \frac{1}{8}\right)$ but much less than $\varepsilon_{*}$. Suppose first that $\left\{\delta_{n}\right\}_{n=1,2, \ldots}$ diverges. Use Lemma 4.10 to find, for any sufficiently large $n$, a set $\left.\Lambda \subset N_{z}\right|_{p}$ of at most $\hat{m}$ points with the following properties: First, each point in $\left.\alpha^{-1}(0) \cap N_{z}\right|_{D_{2 \rho}}$ has distance $\varepsilon^{2} \delta_{n} r_{n}^{-1 / 2}$ or less from $D_{2 \rho} \times \Lambda$. Second, the distance between any two distinct points in $\Lambda$ is greater than $\varepsilon \delta_{n} r_{n}^{-1 / 2}$. Each point in any sufficiently large $n$ version of $\Lambda$ has an associated, positive integer multiplicity, this being the degree of $\alpha /|\alpha|$ on a circle of radius $\frac{1}{4} \varepsilon$ in $\left.N_{z}\right|_{p}$ about the given point. These 
integers sum to $\hat{m}$. When $u \in \Lambda$, use $m_{u}$ to denote its associated integer. It then follows using (3-35) and Lemmas 3.8 and 7.1 that

$$
\rho^{-2} \rho_{\diamond}^{-\widehat{m}}\left|\int_{N_{z}} \chi^{p} \Pi_{u \in \Lambda}(\mathfrak{s}-u)^{m_{u}} F_{\hat{A}} \wedge \kappa_{0} \wedge \bar{\kappa}_{0}\right| \leq c_{0} \varepsilon
$$

when $n$ is large. Given the assumptions of the lemma, there is at least one point in $\rho_{*}^{-1} \Lambda$ with norm greater $\varepsilon_{*}$. This fact with (7-18) and (7-19) implies what is asserted by the lemma. In the case when $\left\{\delta_{n}\right\}_{n=1,2, \ldots}$ converges, Lemma 4.10 and Proposition 4.2 give $\Lambda$ and also (7-19) when $n$ is large. As before, (7-18) and (7-19) imply the lemma's assertion.

The fact that (7-18) holds with $\mathfrak{e}=0$ when the second bullet of the lemma is assumed follows from two facts: First, $\hat{A}=A^{\prime}$ where $|\mathfrak{s}| \geq \frac{1}{2} z$ on $\left.N_{z}\right|_{D_{2 \rho} \rho}$. Moreover, both are flat here, and both are such that $\alpha /|\alpha|$ is covariantly constant. In the case where the first bullet of the lemma is assumed, then the $A^{\prime}$ version of (7-18) holds with $\mathfrak{e}=0$. Meanwhile, it follows from (3-35), Lemma 3.8 and the first item in Lemma 7.1 that the $\widehat{A}$ and $A^{\prime}$ versions of (7-18) differ by no more than $c_{0} e^{-\sqrt{r_{n}} z / \kappa}$.

\section{7.d Convergence on domains where $|s|$ is bounded}

The lemmas from the preceding section are used here to say something about the points in $\alpha_{n}^{-1}(0)$ where the distance to $\bigcup_{(C, m) \in \vartheta} C$ is a sizable fraction of $\delta_{n} r_{n}^{-1 / 2}$.

Lemma 7.5 Given $\varepsilon_{*}>0$ and $T \geq R$, there exists $\kappa>0$ such that if $n \geq \kappa$, then

$$
\sup _{\substack{x \in \alpha_{n}^{-1}(0),|s(z)| \leq T}} \operatorname{dist}\left(\bigcup_{(C, m) \in \vartheta} C, x\right) \leq \varepsilon_{*} \delta_{n} r_{n}^{-1 / 2} .
$$

The remainder of this subsection is occupied with the following.

Proof of Lemma 7.5 Suppose for the sake of argument that this lemma is false. If so, there exists $T \geq R$ and $\varepsilon_{*}>0$ and a subsequence (hence renumbered consecutively from 1) of $\left\{\left(r_{n},\left(A_{n}, \psi_{n}\right)\right)\right\}_{n=1,2, \ldots}$ such that the following is true: For each $n$, there is a point $x_{n}$ where $\alpha_{n}=0$ and with $\left|s\left(x_{n}\right)\right| \leq T$ such that $\operatorname{dist}\left(\bigcup_{(C, m) \in \vartheta} C, z_{n}\right)>$ $\varepsilon_{*} \delta_{n} r_{n}^{-1 / 2}$. Nonsense will now be derived from this assumption. To this end, distinguish now two cases. In the first case, $\left\{\delta_{n}\right\}_{n=1,2, \ldots}$ is bounded. In this case, no generality is lost by assuming that this sequence converges to some $\delta_{0}>0$ and that $\left|\delta_{n}-\delta_{0}\right| \leq \frac{7}{8} \delta_{0}$ for all $n$. This is called the convergent case in what follows. In the second case, $\left\{\delta_{n}\right\}_{n=1,2, \ldots}$ is unbounded. In this case no generality is lost by assuming that the sequence is increasing. This case is called the divergent case. The derivation of the 
desired nonsense has two parts, these depending on whether the sequence $\left\{\delta_{n}\right\}_{n=1,2, \ldots}$ is divergent or convergent.

Part 1 Suppose here that $\left\{\delta_{n}\right\}_{n=1,2, \ldots}$ is divergent. For each $n$, fix an increasing, unbounded sequence $\left\{x_{n}\right\}_{n=1,2, \ldots} \subset[8, \infty)$ but such that the sequence $\left\{x_{n}^{2} \delta_{n} r_{n}^{-1 / 2}\right\}$ converges to zero. One additional growth constraint on $\left\{x_{n}\right\}_{n=1,2, \ldots}$ is given momentarily. For any given such sequence $\left\{\chi_{n}\right\}_{n=1,2, \ldots}$, there exists an increasing, unbounded sequence $\left\{T_{n}\right\}_{n=1,2, \ldots} \subset\{R, \infty\}$ such that the following is true: For each $n$, let $z=z_{n}$ denote the maximum of the numbers $\chi_{n}^{2} \delta_{n} r_{n}^{-1 / 2}$ and $\left(\operatorname{lnr}_{n}\right)^{2} r_{n}^{-1 / 2}$. Then the map $\times_{(C, m) \in \vartheta} e_{C}$ embeds the $|s| \leq T_{n}$ part of $\times_{(C, m) \in \vartheta} N_{z}$.

Given $n$, set $\rho=\rho_{n}=\chi_{n} \delta_{n} r_{n}^{-1 / 2}$, set $z=z_{n}=\max \left\{\chi_{n}^{2} \delta_{n} r_{n}^{-1 / 2},\left(\operatorname{lnr}_{n}\right)^{2} r_{n}^{-1 / 2}\right\}$ and set $\rho_{\diamond}=\rho_{\diamond n}=8 \delta_{n} r_{n}^{-1 / 2}$. Fix $(C, m) \in \vartheta$. If $C$ is not $\mathbb{R}$-invariant (and so $m=1$ ), use (7-10) to define the section $\mathfrak{o}_{1}=\mathfrak{o}_{(n) 1}$ using the function $x \rightarrow \wp(x)=x$ to define $\widehat{A}$ from $\left(A_{n}, \psi_{n}\right)$. If $C$ is $\mathbb{R}$-invariant and $k \in\{1, \ldots, m\}$ use (7-10) to define $\mathfrak{o}_{k}=\mathfrak{o}_{(n) k}$ using the function $x \rightarrow \wp(x)=x \chi(x)+1-\chi(x)$ to define $\widehat{A}$ from $\left(A_{n}, \psi_{n}\right)$. The following is a consequence of Lemma 7.2 and Lemma 7.3: Given $T^{\prime}>R$, there exists $n^{\prime}$ such that the sequence $\left\{\mathfrak{o}_{(n) k}\right\}_{n \geq n^{\prime}}$, is uniformly Holder continuous for any given exponent $v \in(0,1)$ on the $|s| \leq T^{\prime}$ portion of $C$. Moreover, it has bounded $L_{1}^{2}$ norm on this part of $C$. Furthermore, for each $n$ and $k$, the section $\mathfrak{o}_{k}=\mathfrak{o}_{(n) k}$ obeys

- if $m=1$, then $\bar{\partial}_{\theta} \mathfrak{o}_{1}+v_{C} \mathfrak{o}_{1}+\mu_{C} \overline{\mathfrak{o}}_{1}=\mathfrak{e}$,

- if $m>1$, then $\bar{\partial}_{\theta} \mathfrak{o}_{k}+k v_{C} \mathfrak{o}_{k}=\mathfrak{e}$,

where, in each case, $\mathfrak{e}=\mathfrak{e}_{(n)}$ obeys $|\mathfrak{e}| \leq 1 / T^{\prime}$.

Granted this last fact, it follows that the sequence $\left\{\mathfrak{o}_{(n) k}\right\}_{n=1,2, \ldots}$ has a subsequence that converges strongly on compact subsets of $C$ in the $L_{1}^{2}$ topology and in any given $v \in(0,1)$ Holder topology to a bounded section of $N^{\otimes k}$ that obeys the $\mathfrak{e}=0$ version of (7-20). Use $\mathfrak{o}_{k}$ to denote this section.

Now comes the promised extra constraint on the sequence $\left\{\chi_{n}\right\}_{1,2}, \ldots$. By assumption, there exists $\varepsilon_{*}>0$, a subsequence of $\left\{\left(r_{n},\left(A_{n}, \psi_{n}\right)\right)\right\}_{n=1,2, \ldots}$ (hence renumbered consecutively from 1$)$ and $(C, m) \in \vartheta$ with the following property: For each $n$, there is a point, $y_{n}$, in the $|s|<T_{n}-1$ part of the $z=z_{n}$ version of $N_{z}$ where $\alpha_{n}=0$ and with distance at least $\varepsilon_{*} \delta_{n} r_{n}^{-1 / 2}$ from $C$. This understood, it follows from Lemma 7.4 that there exists an increasing, unbounded sequence $\left\{\chi_{0}\right\}_{n=1,2, \ldots}$ such that if $\chi_{n} \leq \chi_{0 n}$, then there is an integer $k \in\{1, \ldots, m\}$ such that $\left|\mathfrak{o}_{(n) k}\right| \geq c>0$ at the point $\pi\left(y_{n}\right) \in C$. Here $c$ depends on $\varepsilon_{*}$ but neither on $n$ nor $y_{n}$. Assume that this growth constraint holds. 
Suppose that there exists, for each $n$, such a point $y_{n} \in C$ as above and that these are such that $\left|s\left(y_{n}\right)\right|$ is bounded. Then there is a subsequence of $\left\{\pi\left(y_{n}\right)\right\}_{n=1,2, \ldots}$ that converges in $C$ to a point $p \in C$, and this point is such that $\left.\mathfrak{o}_{k}\right|_{p} \neq 0$. Thus, $\mathfrak{o}_{k}$ is a nontrivial, bounded solution to the $\mathfrak{e}=0$ version of (7-20) on the whole of $C$. This conclusion is nonsense because the assumptions in [9, Section 4.b] prohibit such solutions.

Part 2 This part argues the case for Lemma 7.5 when $\left\{\delta_{n}\right\}_{n=1,2, \ldots}$ converges. Let $\delta_{0}>0$ denote the limit in what follows. Choose an increasing, unbounded sequence $\left\{\chi_{n}\right\}$ as in Part 1. Use $\rho=\rho_{n}=\chi_{n} \delta_{n} r_{n}^{-1 / 2}$ with $z=z_{n}=r_{n}^{-1 / 2}\left(\operatorname{lnr}_{n}\right)^{2}$ and $\rho_{\diamond}=$ $\rho_{\diamond n}=8 \delta_{n} r_{n}^{-1 / 2}$ to construct for each $(C, m) \in \vartheta$, for each $k \in\{1, \ldots, m\}$, and a section $\mathfrak{o}_{(n) k}$ of $N^{\otimes k}$. Here again, use for $\wp$ the function $x \rightarrow \wp(x)=x$ when $C$ is not $\mathbb{R}$-invariant, but use the function $\wp(x)=x \chi(x)+1-\chi(x)$ otherwise. It is a consequence of Lemmas 7.2, 7.3 and 7.4 that the sequence $\left\{\mathfrak{o}_{(n) k}\right\}_{k=1,2, \ldots}$ converges on compact subsets of $C$ to a section $\mathfrak{o}_{k}$ of $N^{\otimes k}$. This section is a priori bounded and uniformly Holder continuous on $C$ for any given Holder exponent in $(0,1)$. Moreover, if $\left\{x_{n}\right\}_{n=1,2, \ldots}$ does not grow too fast, then there is a point $p \in C$ where $\mathfrak{o}_{k} \neq 0$. As is explained momentarily, the section $\mathfrak{o}_{k}$ also obeys the $\mathfrak{e}=0$ version of (7-20). Given that it is bounded on $C$, this conclusion is also nonsense.

If $C$ is $\mathbb{R}$-invariant, then the fact that $\mathfrak{e}=0$ follows from the bound given in the final assertion of Lemma 7.2 because the latter finds the integer $n$ version of the right hand side of (7-20) bounded by $\kappa\left(\rho_{\diamond n}^{2}+\delta_{0} r_{n}^{-1 / 2}\right)$.

Granted the preceding, it remains only to discuss the case that $C$ is not $\mathbb{R}$-invariant. Here, $m=1$ so $k=1$. It follows from what is said in Steps 1 and 2 of the proof of Lemma 7.1 that the section $\mathfrak{o}_{1}$ will obey the $\mathfrak{e}=0$ version of (7-20) if the following is true: Let $\mathfrak{e}_{(n)}$ denote the integer $n$ version of

$$
\rho^{-2} \rho_{\diamond}^{-1} \int_{\mathbb{C} \times D_{2 \rho}} \chi^{p} \bar{f}_{+} d z \wedge d \bar{z} \wedge d u \wedge d \bar{u}
$$

Then $\lim _{n \rightarrow \infty} \sup _{|s| \leq T_{n}-1}\left|e_{(n)}\right|=0$. The proof that this happens is given momentarily. What follows directly a digression for two observations The digression makes no assumptions about the integer $m$ or $k \in\{1, \ldots, m\}$.

Observation 1 The observation here is summarized in the upcoming Lemma 7.6. To set the stage, note that the sections $\left\{\mathfrak{o}_{1}, \ldots, \mathfrak{o}_{m}\right\}$ that are obtained as limits of the $m$ sequences $\left.\left\{\mathfrak{o}_{(n) k}\right\}_{n=1,2, \ldots}\right\}_{1 \leq k \leq m}$ define a section over $\mathbb{C}$ of the vortex bundle $\mathfrak{C}_{N, m}$. Let $\mathfrak{c}$ denote this section. This section $\mathfrak{c}$ is uniformly Holder continuous on $C$ for any exponent $v \in(0,1)$. It also has finite $L_{1}^{2}$ norm on any compact subset of $C$. 
Moreover, given $\varepsilon>0$ and $T^{\prime}>R$, there is a smooth section $\mathfrak{c}_{\varepsilon, T^{\prime}}$ of $\mathfrak{C}_{N, m}$ and a section, $\xi_{\varepsilon, T^{\prime}}$, of $\left(\mathfrak{c}_{\varepsilon, T^{\prime}}\right)^{*} V_{1,0} \mathfrak{C}_{N, m}$ with the following properties: First, $\left\|\xi_{\varepsilon, T^{\prime}}\right\|_{\infty}<\varepsilon$ and $\varepsilon$ bounds both the $L_{1}^{2}$ norm of $\xi_{\varepsilon^{\prime}, T^{\prime}}$ over the $|s| \leq T^{\prime}$ part of $C$ and the $\mathcal{K}_{*}$ norm of $\xi_{\varepsilon, T^{\prime}}$ as defined in [9, (2-27)] with $C$ replaced by its $|s| \leq T^{\prime}$ part. Finally, $\mathfrak{c}=\exp _{\mathfrak{c}_{\varepsilon^{\prime}, T^{\prime}}}\left(\xi_{\mathcal{E}^{\prime}, T^{\prime}}\right)$.

Fix $n$ large, and let $\rho=x_{n} \delta_{n} r_{n}^{-1 / 2}$ as before. Let $\rho \in C$ and let $D_{\rho} \subset D$ denote the embedded disk of radius $\rho$ centered at $p$. Assume that $n$ is large and so the $z=r_{n}^{-1 / 2}\left(\operatorname{lnr}_{n}\right)^{2}$ version of $\left.N_{z}\right|_{D_{2 \rho}}$ has no intersections with $\alpha_{n}^{-1}(0)$ where $|\mathfrak{s}| \in\left[\frac{1}{8} z, 2 z\right]$. Use parallel transport via the connection $\theta$ along the radial geodesics from $p$ to write the bundle $N$ over $D_{2 \rho}$ as $D_{2 \rho} \times \mathbb{C}$. This done, lift $\mathfrak{c}$ as a pair $\left(\theta+\frac{1}{2}\left(a^{1} d \bar{z}-\bar{a}^{1} d z\right), \alpha^{1}\right)$ where $a^{1}$ and $\alpha^{1}$ are complex valued functions on $D_{2 \rho} \times \mathbb{C}$ such that the pair $\left(A=\frac{1}{2}\left(a^{1} d \bar{z}-\bar{a}^{1} d z\right), \alpha^{1}\right)$ obeys the vortex equations (1-4) on each fiber $\mathbb{C}$. Use $\left(a^{r}, \alpha^{r}\right)$ to denote the pullback of $\left(a^{1}, \alpha^{1}\right)$ by the map from $D_{2 \rho} \times \mathbb{C}$ to itself that sends $(u, z)$ to $\left(u, r_{n}^{1 / 2} z\right)$.

The lemma that follows is an analog of sorts to Lemma 6.3. This lemma reintroduces $\left\{\bar{\partial}_{A 0}, \bar{\partial}_{A 1}\right\}$; these being the respective covariant derivatives for any given connection $A$ along the vector fields that are dual to the basis $\left\{\bar{\kappa}_{0}, \bar{\kappa}_{1}\right\}$ for $\left.T^{0,1}(\mathbb{R} \times M)\right|_{N}$.

Lemma 7.6 There exists an increasing, unbounded sequence $\left\{\chi_{0 n}\right\}_{n=1,2, \ldots}$, such that if each $\chi_{n} \leq \chi_{0 n}$ for each $n$, then the following is true: Fix $\varepsilon>0, T^{\prime} \geq R$ and then $n$ sufficiently large. Fix $p \in C$ where $|s| \leq T^{\prime}$. There is a smooth map $u_{n}: D_{2 \rho} \times \mathbb{C} \rightarrow$ $U(1)$ such that $\left(A_{n}-u_{n}^{-1} d u_{n}, u_{n} \alpha_{n}\right)$ can be written on the $|z| \leq \varepsilon^{-1} r_{n}^{-1 / 2}$ portion of $D_{2 \rho} \times \mathbb{C}$ as

$$
A_{n}-u_{n}^{-1} d u_{n}=\theta+\frac{1}{2} r_{n}^{1 / 2}\left(a^{r} d \bar{u}-\bar{a}^{r} d u\right)+c_{n} \quad \text { and } \quad u_{n} \alpha_{n}=\alpha^{r}+\varsigma_{n},
$$

where $\left|\varsigma_{n}\right|+r_{n}^{-1 / 2}\left(\left|\nabla_{\theta} \varsigma_{n}\right|+\left|c_{n}\right|\right) \leq \varepsilon$.

Proof of Lemma 7.6 This follows as a direct consequence of Lemmas 4.3 and 4.10.

Observation 2 Fix $\varepsilon>0$ and use $r$ in what follows to denote $r_{n}$; and use $(A,(\alpha, \beta))$ to denote $\left(A_{n}-u_{n}^{-1} d u_{n},\left(u_{n} \alpha_{n}, u_{n} \beta_{n}\right)\right)$ where $u_{n}$ is given by Lemma 7.6. The integer $N$ version of the function $\bar{f}_{+}$that appears in (7-21) has the form given in (7-16).

To say something about the size of the contributions to (7-21) from the three terms in (7-16), note first that

- $\left|\bar{\partial}_{A_{n} 0} \alpha_{n}\right| \leq c_{0} \varepsilon r^{1 / 2}\left(e^{-\sqrt{r}|z| / c_{0}}+r^{-1}\right)$

- $\left|\bar{\partial}_{A} \alpha\right| \leq c_{0}\left(e^{-\sqrt{r}|z| / c_{0}}+r^{-1}\right)$

- $|\beta| \leq c_{0} r^{-1 / 2}\left(e^{-\sqrt{r}|z| / c_{0}}+r^{-1}\right)$ 
on the $|z| \leq \frac{3}{4} z$ part of $D_{2 \rho} \times \mathbb{C}$. Indeed, the first item follows from Lemma 7.6 and Lemma 3.8. The second item follows from Lemma 3.8 because the Dirac equation writes the $d$-bar derivatives of $\alpha$ in terms of the derivatives of $\beta$. The third item also quotes from Lemma 3.8.

With the digression now over, return to the proof that the $k=1$ version of $\mathfrak{e}$ is zero. To start, note that (7-15), (7-22) and Lemma 7.6 imply the following: The integral in (7-21) can be written as

$$
\begin{array}{r}
\rho^{-2} \rho_{\diamond}^{-1} \int_{\mathbb{C} \times D_{2 \rho}} \chi^{p} \chi_{z}\left(r\left(1-\left|\alpha^{r}\right|^{2}\right) \bar{\alpha}_{r} \beta+\bar{\partial}_{A^{r} 1^{1}} \bar{\alpha}^{r} \partial_{A^{r} 1} \beta\right) \\
\times d z \wedge d \bar{z} \wedge d u \wedge d \bar{u}+\mathfrak{r},
\end{array}
$$

where $|\mathfrak{r}| \leq c_{0} \varepsilon$. Note that the derivation of (7-23) invokes the Dirac equation to write $\bar{\partial}_{A 0} \alpha$ as $-\bar{\partial}_{A 1} \beta$. To say something about the integral in (7-23), integrate by parts now to take the derivative $\bar{\partial}_{A^{r} 1}$ off of $\bar{\alpha}^{r}$ and put it on $\partial_{A^{r}{ }_{1}} \beta$. Doing so adds a term with a derivative of $\chi_{z}$, thus a term with norm bounded by $r^{-1}$ when $n$ is large. Now commute these two derivatives on $\beta$ and integrate by parts to leave just one derivative, $\bar{\partial}_{A^{r} 1}$, on $\beta$. The result of all of these manipulations is an integral whose integrand has a factor with a derivative on $\chi_{z}$. As a consequence, the resulting integral has norm bounded by $r^{-1}$ when $n$ is large. This then proves that $\left|\mathfrak{e}_{(n) 1}\right| \leq c_{0} \varepsilon$ when $n$ is large.

\section{7.e On the ends of $C$}

The next lemma gives the large $|s|$ analog of what is asserted by Lemma 7.5. These lemmas together imply the following: Fix $\varepsilon_{*}>0$ and all sufficiently large $n$ versions of $\delta_{n}$ are less than $\varepsilon_{*} \delta_{n}$. This is, of course, impossible. This nonsense proves Lemma 6.2.

Lemma 7.7 Given $\varepsilon_{*}>0$ and $T \geq R$, there exists $\kappa>0$ such that if $n \geq \kappa$, then

$$
\sup _{\substack{z \in \alpha_{n}^{-1}(0),|s(z)| \geq T}} \operatorname{dist}\left(\bigcup_{(C, m) \in \vartheta} C, z\right) \leq \varepsilon_{*} \delta_{n} r_{n}^{-1 / 2} .
$$

Proof of Lemma 7.7 Each Reeb orbit in either $\Xi_{\Sigma-}$ or $\Xi_{\Sigma+}$ has a tubular neighborhood map as described in [9, (4-1)] with coordinates $(w, t, z)$ for $\mathbb{R} \times S^{1} \times D$. The latter map is used implicitly in what follows.

If Lemma 7.7 is false, then there exists $\varepsilon_{*}>0$, a Reeb orbit $\gamma \in \Xi_{\Sigma-}$ or $\Xi_{\Sigma+}$ and a subsequence of $\left\{\left(r_{n},\left(A_{n}, \psi_{n}\right)\right)\right\}_{n=1,2, \ldots}$ (hence renumbered consecutively) with the following property: Fix $T \geq R$. Suppose that $\gamma \in \Xi_{\Sigma-}$. If $n$ is sufficiently large, there is a point in $\gamma$ 's version of $(-\infty,-2 T] \times S^{1} \times D$ where $\alpha_{n}=0$ and with distance 
at least $\varepsilon_{*} \delta_{n} r_{n}^{-1 / 2}$ from $\bigcup_{(C, m) \in \vartheta} C$. If $\gamma \in \Xi_{\Sigma+}$, then the same conclusion holds except that the point in question lies in $\gamma$ 's version of $[2 T, \infty) \times S^{1} \times D$. Assume in what follows that $\gamma \in \Xi_{\Sigma-}$ so as to derive some nonsense. But for some cosmetic changes, the same argument will derive nonsense in the case where $\gamma \in \Xi_{\Sigma+}$. This understood, the latter case is not discussed further. The derivation of nonsense for the case $\gamma \in \Xi_{\Sigma-}$ presented in next in three parts.

Part 1 This part considers the case when $\gamma$ is such that $m_{\gamma-}=1$. In particular, this part deals with the case when $\gamma$ is hyperbolic. Given $T \gg R$ and then $n$ sufficiently large, Lemma 7.5 say that there are no points in $\alpha_{n}^{-1}(0) \cap\left([-2 T,-T] \times S^{1} \times D\right)$ with distance greater than $\varepsilon_{*} \delta_{n} r_{n}^{-1 / 2}$ from $C$. Even so, there is a point $y_{n} \in \alpha_{n}^{-1}(0) \cap$ $\left([-\infty,-2 T) \times S^{1} \times D\right)$ such that $\operatorname{dist}\left(y_{n}, C\right) \geq \varepsilon_{*} \delta_{n} r_{n}^{-1 / 2}$. It follows from Lemma 7.5 that the sequence $\left\{s\left(y_{n}\right)\right\}_{n=1,2, \ldots}$ has no convergent subsequence.

Construct from $\left(A_{n}, \psi_{n}\right)$ the section $\mathfrak{o}_{(n) 1}$ as described in Lemma 7.2. Note that $\mathfrak{o}_{(n) 1}$ is defined on the whole of the end $\mathcal{E}$ of $C$ in $(-\infty,-T] \times S^{1} \times D$ Trivialize the normal bundle to $C$ on $\mathcal{E}$ by the vector field $\partial / \partial z$ to view $\mathfrak{o}_{(n) 1}$ as a map from $(-\infty,-T] \times S^{1}$ to $\mathbb{C}$. Let $\mathfrak{q}_{(n) 1}:\left(-\infty,-T-w\left(y_{n}\right)\right] \times S^{1} \rightarrow \mathbb{C}$ denote the map sending $u=w+i t$ to $\left.\mathfrak{o}_{(n) 1}\right|_{u+w_{n}}$.

The resulting sequence $\left\{\mathfrak{q}_{(n) 1}\right\}_{n=1,2, \ldots}$ has a subsequence that converges on compact subsets of $\mathbb{R} \times S^{1}$ in the Holder topology with any given exponent in $(0,1)$ to a map $\mathfrak{q}_{1}$ from $\mathbb{R} \times S^{1}$ to $\mathbb{C}$ that has uniformly bounded $L_{1}^{2}$ norm on the cylinders $\left\{[n, n+2] \times S^{1}\right\}_{n \in \mathbb{Z}}$, is uniformly Holder continuous for any given exponent in $(0,1)$, and is nonzero at some point on $\{0\} \times S^{1}$. Moreover, the argument given in Parts 1 and 2 of the proof of Lemma 7.5 can be employed with no changes to see that $\mathfrak{q}_{1}$ obeys $\bar{\partial}_{\theta} \mathfrak{q}_{1}+v \mathfrak{q}_{1}+\mu \overline{\mathfrak{q}}_{1}=0$ on the whole of $\mathbb{R} \times S^{1}$. However, this is impossible as there are no nontrivial, uniformly bounded solutions to this equation.

Part 2 Suppose now that $m_{\gamma-}>1$. To keep notation to a minimum, assume in what follows that $\ell_{\gamma}=2 \pi$. This assumption has no bearing on the arguments and can be eliminated at the cost of inserting a factor of $\ell_{\gamma} /(2 \pi)$ or its inverse in many of the subsequent equations.

Introduce from $\left[9\right.$, Section 5.c] the partition $\left\{\mathcal{E}_{1}, \ldots, \mathcal{E}_{N}\right\}$ of the ends of $\bigcup_{(C, m) \in \vartheta} C$ that lie in $U_{\gamma-}$. The argument that follows is simplest in the case when there is but one of these sets, thus $N=1$. In this regard, the algebra is somewhat simpler when there is also just one end in $\mathcal{E}_{1}$. The reader might find the going easier by assuming that such is the case at first reading. In any event, the rest of this Part 2 considers the case when $N=1$. The argument for this case has three steps.

Note that $m$ is used as shorthand for $m_{\gamma-}$ in these steps. 
Step 1 Suppose for the moment that the ends in $\mathcal{E}_{1}$ are not part of $\mathbb{R} \times \gamma$. (Note that $\mathcal{E}_{1}$ has but a single end if it has any from $\mathbb{R} \times \gamma$.) Each $\mathcal{E} \in \mathcal{E}_{1}$ has an associated multiplicity, $q_{\mathcal{E}} \in \mathbb{Z}$. These are the same for all ends in $\mathcal{E}_{1}$. Introduce, as in [9, (4-1)], the local coordinate $u=w+i t$ with $(w, t)$ the coordinates of $\mathbb{R} \times S^{1}$. It follows from $[9,(4-2)]$ that any given end $\mathcal{E}$ from $\mathcal{E}_{1}$ appears in the $w \leq w_{0} \ll$ -1 portion of $\mathbb{R} \times S^{1} \times D$ as a $q$-sheeted graph over $\left(-\infty, w_{0}\right] \times S^{1}$ with sheets parameterized (locally) as the graph of a multivalued function $u \rightarrow z_{\mathcal{E}}(u)$. Here, $z_{\mathcal{E}}(u)=\varsigma_{\mathcal{E}} e^{-\mathrm{R} w+k_{\mathcal{E}} u / q_{\mathfrak{E}}}\left(1+\mathfrak{r}_{\mathfrak{E}}\right)$ where $\varsigma_{\mathcal{E}} \in \mathbb{C}-0$ and $k_{\mathcal{E}} \in \mathbb{Z}$ is relatively prime to $q_{\mathcal{E}}$ and obeys $\left(k_{\mathcal{E}}-1\right) / q_{\mathcal{E}}<\mathrm{R}<k_{\mathcal{E}} / q_{\mathcal{E}}$. Meanwhile $\left|\mathfrak{r}_{\mathfrak{E}}\right| \leq \frac{1}{100} e^{-|w| / c_{0}}$. This function $z_{\mathcal{E}}(\cdot)$ also obeys the equation $(\partial / \partial \bar{u}) z_{\mathcal{E}}+\mathrm{R} z_{\mathcal{E}}=0$. Let $\mathfrak{Z}_{\mathcal{E}}(u) \subset \mathbb{C}$ denote the set of $q_{\mathcal{E}}$ values of $z_{\mathcal{E}}(u)$. As a final remark, note that the collection $\left\{\zeta_{\mathcal{E}}\right\}_{\mathcal{E} \in \mathcal{E}_{1}}$ is such that $\varsigma_{\mathcal{E}} / \varsigma_{\mathcal{E}^{\prime}}$ can not be a $q_{\mathcal{E}}$-th root of unity unless $\mathcal{E}=\mathcal{E}^{\prime}$.

If a counterexample to the assertion made by Lemma 6.2 is to appear here, then there is an infinite subsequence of $\left\{\left(r_{n},\left(A_{n}, \psi_{n}\right)\right)\right\}_{n=1,2, \ldots}$ with the following property: There exists $\left(u_{n}=w_{n}+i t_{n}, z_{n}\right) \in\left(-\infty,-w_{0}\right] \times S^{1} \times D$ where $\alpha_{n}=0$ and such that

$$
\left|z_{n}-z^{\prime}\right| \geq \varepsilon_{*} \delta_{n} r_{n}^{-1 / 2} \quad \text { for all } z^{\prime} \in \mathfrak{Z}_{\mathcal{E}}\left(u_{n}\right) \text { and all } \mathcal{E} \in \mathcal{E}_{1} \text {. }
$$

Here are two cases to consider with regards to (7-24):

- There exists $\varepsilon>0$ and a subsequence of $\left\{\left(r_{n},\left(A_{n}, \psi_{n}\right)\right\}_{n=1,2, \ldots}\right.$ with a corresponding $\left(u_{n}, z_{n}\right) \in \alpha_{n}^{-1}(0)$ such that (7-24) holds with $\varepsilon e^{\left(-\mathrm{R}+k_{\mathcal{E}} / q_{\mathcal{E}}\right) w_{n}}<\delta_{n} r_{n}^{-1 / 2}$.

- Fix any $\varepsilon>0$. If $n$ is sufficiently large, then any point $\left(u_{n}, z_{n}\right) \in \alpha_{n}^{-1}(0)$ that obeys (7-24) is such that $\varepsilon e^{\left(-\mathrm{R}+k_{\mathcal{E}} / q_{\mathfrak{E}}\right) w_{n}}>\delta_{n} r_{n}^{-1 / 2}$.

If $\mathcal{E}$ is part of an $\mathbb{R}$-invariant cylinder, and if $\mathcal{E}$ is to provide a counterexample to the assertion made by Lemma 6.2, then there is a subsequence of $\left\{\left(r_{n},\left(A_{n}, \psi_{n}\right)\right)\right\}_{n=1,2, \ldots}$ where (7-24) holds with $z_{\mathcal{E}}(\cdot)=0$.

Step 2 Assume that either the first item in (7-25) holds, or else $\mathcal{E}$ is part of an $\mathbb{R}$-invariant cylinder and so (7-24) holds with $z_{\mathcal{E}}=0$. Pass to a refined subsequence for which the corresponding sequence $\left\{\delta_{n}\right\}_{n=1,2, \ldots}$ either converges, or is increasing and unbounded. Relabel the subsequence in question consecutively from 1. Such a subsequence can and should be chosen so that after this relabeling, both $e^{\left(-\mathrm{R}+k_{\mathcal{E}} / q_{\mathcal{E}}\right) w_{n}} \leq n^{-4}$ and $w_{n}<-n$. Require in addition that the associated sequence $\left\{\left(\delta_{n} r_{n}^{-1 / 2}\right)^{-1} e^{\left(-\mathrm{R}+k_{\mathcal{E}} / q_{\mathcal{E}}\right) w_{n}}\right\}_{n=1,2, \ldots}$ converges. Note that the first item in (7-25) guarantees that this is a bounded sequence.

For each $n$, construct sections $\left\{\mathfrak{o}_{(n) k}\right\}_{1 \leq k \leq m}$ as described by Lemma 7.2 and (7-9) using for $C$ the $w \in\left[w_{n}-\left(1 /\left(50 q^{2}\right)\right) \ln (n), w_{n}+\left(1 /\left(50 q^{2}\right)\right) \ln (n)\right]$ portion of the 
cylinder $\mathbb{R} \times \gamma$. To elaborate, the construction is done using the following data: The connection $\hat{A}$ is defined from $\left(A_{n}, \psi_{n}\right)$ using the function $\wp$ given by the rule $\wp(x)=x \chi(x)+1-\chi(x)$. Take $\rho=\rho_{n}=100 \delta_{n} r_{n}^{-1 / 2}$ and take $z=z_{n}=$ $100 n \delta_{n} r_{n}^{-1 / 2}$. Finally, take $\rho_{\diamond}=\rho{ }=16 \delta_{n} r_{n}^{-1 / 2}$. The vector field $\partial / \partial z$ trivializes the normal bundle to $C$ here, and this understood, view each $\mathfrak{o}_{(n) k}$ as a $\mathbb{C}$-valued function. For each $n$ and $k \in\{1, \ldots, m\}$, define the function $\mathfrak{q}_{(n) k}$ with domain $\left[-\left(1 /\left(50 q^{2}\right)\right) \ln (n),\left(1 /\left(50 q^{2}\right)\right) \ln (n)\right] \times S^{1} \subset R \times S^{1}$ by setting $\left.\mathfrak{q}_{(n) k}\right|_{u}=\left.\mathfrak{o}_{(n) k}\right|_{u+w_{n}}$.

As is explained next, there exists $\kappa_{*} \geq 50 \mathrm{~m}^{2}$ which guarantees the following when $n$ is large:

(7-26) $\left|\alpha_{n}\right|^{2} \geq \frac{1}{2}$ at all $(u, z) \in\left[w_{n}-\frac{1}{\kappa_{*}} \ln (n), w_{n}+\frac{1}{\kappa_{*}} \ln (n)\right] \times S^{1} \times D,|z| \geq \frac{1}{4} z_{n}$.

To see why this is true, note first that $z$ must have distance $2 \delta_{n} r_{n}^{-1 / 2}$ or less from a point $z^{\prime} \in \mathfrak{Z}_{E}(u)$. Such a point $z^{\prime}$ obeys $\left|z^{\prime}\right| \leq c_{0} e^{\left(-\mathrm{R}+k_{\mathcal{E}} / q_{\mathcal{E}}\right) w}$, and this is less than $c_{0} n^{1 / 2} e^{\left(-\mathrm{R}+k_{\mathcal{E}} / q\right) w_{n}}$ if $u$ is such that $w \leq c_{0}^{-1} \ln (n)$ for a suitable choice of $c_{0}$. By virtue of what is assumed by the first item in (7-25), the latter is less than $c_{0} \varepsilon^{-1} n^{1 / 2} \delta_{n} r_{n}^{-1 / 2}$, and so $|z| \leq n^{-1 / 4} z_{n}$ when $n$ is large.

What with (7-26), the final bullet of Lemma 7.2 can be invoked to see that

$$
\left|\mathfrak{q}_{(n) k}\right| \leq c_{0}\left(1+e^{-\left(-\mathrm{R}+k_{\mathcal{E}} / q_{\mathcal{E}}\right) w}\right)^{k} \quad \text { and } \quad \bar{\partial} \mathfrak{q}_{(n) k}+\frac{1}{2} k \mathrm{R} \mathfrak{q}_{(n) k}=\mathfrak{e}_{n},
$$

where $\left\{\left|\mathfrak{e}_{(n)}\right|\right\}_{n=1,2, \ldots}$ has limit zero. As in the proof of Lemma 7.3, these bounds imply that each $k \in\{1, \ldots, m\}$ version of $\left\{\mathfrak{q}_{(n) k}\right\}_{n=1,2, \ldots}$ has a subsequence that converges on compact subsets of $\mathbb{R} \times \gamma$ in any given Holder norm to a function, $\mathfrak{q}_{k}$, that obeys

$$
\left|\mathfrak{q}_{k}\right| \leq c_{0}\left(1+e^{-\left(-\mathrm{R}+k_{\mathcal{E}} / q_{\mathcal{E}}\right) w}\right)^{k} \quad \text { and } \quad \bar{\partial} \mathfrak{q}_{k}+\frac{1}{2} k \mathrm{R} \mathfrak{q}_{k}=0 .
$$

These subsequences can and should be chosen so as to guarantee that $\left\{\mathfrak{q}_{k}\right\}_{1 \leq k \leq m}$ enjoys one additional property. To

elaborate, let $h$ denote the number of ends in $\mathcal{E}_{1}$. Thus, $m=h q_{\mathcal{E}}$. Also, let $c$ denote the limit of the $\left\{\left(16 \delta_{n} r_{n}^{-1 / 2}\right)^{-1} e^{\left(-\mathrm{R}+k_{\mathcal{E}} / q_{\mathcal{E}}\right) w_{n}}\right\}_{n=1,2, \ldots}$. Here is the extra property:

$$
\text { Either } \mathfrak{q}_{k} \neq 0 \text { for some } k \neq 0 \bmod \left(q_{\mathcal{E}}\right) \text {, }
$$

or there exists $x \in\{1, \ldots, h\}$

$$
\text { such that }\left.\mathfrak{q}_{k=x q_{\mathcal{E}}}\right|_{\mathfrak{u}} \neq \mathfrak{c}^{x q_{\mathbb{E}}}\left(\sum_{\mathcal{E} \in \mathcal{E}_{1}} \varsigma_{\mathcal{E}}^{x q_{\mathcal{E}}}\right) e^{-x\left(q_{\mathfrak{E}} \mathrm{R} w+k_{\mathfrak{E}} u\right)}
$$

To see why this is true, recall $z_{\mathcal{E}}(u)=\varsigma_{\mathcal{E}} e^{-\mathrm{R} w+k_{\mathfrak{E}} u / q_{\mathcal{E}}}\left(1+\mathfrak{r}_{\mathfrak{E}}\right)$ with $\left|\mathfrak{r}_{\mathfrak{E}}\right| \leq \frac{1}{100} e^{-|w| / c_{0}}$. With this in mind, note that the first item in (7-25) finds $e^{-\left|w_{n}\right| / c_{0}} e^{\left(-\mathrm{R}+k_{\mathcal{E}} / q_{\mathcal{E}}\right) w_{n}} \leq$ 
$n^{-4} \delta_{n} r_{n}^{-1 / 2}$ when $n$ is large. This and (7-24) imply that $\left|z_{n}-z^{\prime}\right| \geq \frac{1}{2} \varepsilon_{*} \delta_{n} r_{n}^{-1 / 2}$ for any $z^{\prime} \in D$ that has the form $\eta \varsigma_{\mathcal{E}} e^{-\mathrm{R} w_{n}+k_{\mathcal{E}} u_{n} / q_{\mathcal{E}}}$ with $\mathcal{E} \in E_{1}$ and with $\eta^{q}=1$. This guarantees the existence of subsequences whose limits obey (7-29).

To see what (7-28) and (7-29) imply, remark that a solution to the right hand equation in (7-28) is a linear combination of functions that have the form $e^{-k \mathrm{R} w+j u}$ for some $j \in \mathbb{Z}$. A function of this sort obeys the left hand inequality in (7-28) if and only if $\mathrm{R}<j / k \leq k_{\mathcal{E}} q_{\mathcal{E}}$. (It follows from [2, Lemma 6.11] that no such $j$ exists unless $k$ is divisible by $q_{\mathcal{E}}$, but this fact is not needed for what follows.) The other possibility has $k=x q_{\mathcal{E}}$ and $j=x k_{\mathcal{E}}$ for some $x \in\{1, \ldots, h\}$. Thus, $\mathfrak{q}_{x q_{\mathcal{E}}}=\varsigma_{x} e^{-x\left(q_{\mathcal{E}} \mathrm{R} w+k_{\mathcal{E}} u\right)}$ for some $\varsigma_{x} \in \mathbb{C}$. Thus, in each case, $\mathfrak{q}_{k}$ is a finite polynomial.

Introduce the two $u$-dependent polynomials

- $\left.\mathcal{P}(\lambda)\right|_{u}=\lambda^{m}+\sum_{1 \leq k \leq m} \mathfrak{q}_{k}(u) \lambda^{m-k}$.

- $\mathcal{P}_{0}(\lambda)=\lambda^{m}+\sum_{1 \leq x \leq h} \mathfrak{c}^{x q_{\mathcal{E}}}\left(\sum_{\mathcal{E} \in E_{1}} \varsigma_{\mathcal{E}}^{x q_{\mathcal{E}}}\right) e^{-x\left(q_{\mathbb{E}} \mathrm{R} w+k_{\mathbb{E}} u\right)} \lambda^{m-x q_{\mathcal{E}}}$.

These two polynomials differ. In particular, the following is a consequence of what was said in the preceding paragraph about the possibilities for $\left\{\mathfrak{q}_{k}(u)\right\}_{1 \leq k \leq m}$ :

Given $\Delta>0$, there exists $w_{\Delta}$ such that if $u$ has real part greater than $w_{\Delta}$, then $\left.\mathcal{P}(\lambda)\right|_{u}$ has a root with distance greater than $\Delta$ from each root of $\mathcal{P}_{0}(\lambda)$.

Indeed, (7-31) follows from what [10, Part 6 of Section 3.a] says for the proof of [10, Lemma 3.2], and from what is said in [9, Section 7.i] for the proof of [9, Lemma 7.5].

Given that each sequence $\left\{\mathfrak{q}_{(n) k}\right\}_{n=1,2, \ldots}$ converges pointwise on bounded domains to $\mathfrak{q}_{k}$, what is said by (7-31) requires the following for all large $n$ : There exists points $(u, z) \in \alpha_{n}^{-1}(0)$ such that $\left|z-z^{\prime}\right|>16 \Delta \delta_{n} r_{n}^{-1 / 2}$ for all $z^{\prime} \in \mathfrak{Z}_{\mathcal{E}}(u)$ and all $\mathcal{E} \in \mathcal{E}_{1}$ when $\Delta>c_{0}$. Of course, this is nonsense because any such $(u, z)$ must have distance $\delta_{n} r_{n}^{-1 / 2}$ or less from some point in some end $\mathcal{E} \in \mathcal{E}_{1}$.

Step 3 This step considers the case where the second item in (7-25) holds. To start, fix a subsequence of $\left\{\left(r_{n},\left(A_{n}, \psi_{n}\right)\right)\right\}_{n=1,2, \ldots}$ and renumber consecutively from 1 so that $\left\{\delta_{n}\right\}_{n=1,2, \ldots}$ is either unbounded or converges. This sequence can and should be chosen so that the following is also true: If (7-24) holds for any given $\left(u_{n}, z_{n}\right) \in \alpha_{n}^{-1}(0)$, then $e^{\left(-\mathrm{R}+k_{\mathcal{E}} / q_{\mathbb{E}}\right) w_{n}}>e^{n} \delta_{n} r_{n}^{-1 / 2}$.

Fix an increasing, unbounded sequence $\left\{\chi_{n}\right\}_{n=1,2, \ldots} \subset[8, \infty)$ but such that the sequence $\left\{\chi_{n}^{2} \delta_{n} r_{n}^{-1 / 2}\right\}$ converges to zero. Choose $x_{n} \leq n$. One additional upper bound on the growth of the sequence $\left\{\chi_{n}\right\}_{n=1,2, \ldots}$ arises below. Set $\rho_{n}=\chi_{n} \delta_{n} r_{n}^{-1 / 2}$ and set 
$z_{n}=n^{2} \delta_{n} r_{n}^{-1 / 2}$. This choice for $\left\{z_{n}\right\}_{n=1,2, \ldots}$ guarantees the existence of a constant $c \geq 1$ with the following property:

Let $\Sigma_{n}$ denote the $w \in\left[w_{n}-(1 / c) \ln (n), w_{n}+(1 / c) \ln (n)\right]$ part of $\bigcup_{\mathcal{E} \in \mathcal{E}_{1}} \mathcal{E}$. If $n$ is large, then the exponential map embeds the radius $2 z_{n}$ disk bundle in $\left.N_{1}\right|_{\Sigma_{n}}$.

With $\rho_{n}$ and $z_{n}$ understood, set $\rho_{\diamond n}=16 \delta_{n} r_{n}^{-1 / 2}$.

Fix $n$ large, set $\rho=\rho_{n}, z=z_{n}$ and $\rho_{\diamond}=\rho_{\diamond n}$, and then mimic what is done when $k=1$ in Parts 1 and 2 of the proof of Lemma 7.5 to construct over $\Sigma_{n}$ a section, $\mathfrak{o}_{(n) 1}$, of the normal bundle $N$. However, use now the function $\wp$ given by $\wp(x)=x \chi(x)+1-\chi(x)$ to define $\hat{A}$.

Use the product structure on $\left.N\right|_{\Sigma_{n}}$ given by the vector field $\partial / \partial z$ to view $\mathfrak{o}_{(n) 1}$ as a complex function on each component of $\Sigma_{n}$. Fix such a component, and use the local parameterization of any given $\mathcal{E} \in \mathcal{E}_{1}$ as the graph of $z_{\mathcal{E}}(\cdot)$ to view $\mathfrak{o}_{(n) 1}$ on the given component as a $\mathbb{C}$-valued function on $\left[w_{n}-(1 / c) \ln (n), w_{n}+(1 / c) \ln (n)\right] \times\left(\mathbb{R} /\left(2 \pi q_{\mathcal{E}} \mathbb{Z}\right)\right)$. With this view understood, define $\mathfrak{q}_{(n) 1}$ by the rule $\left.\mathfrak{q}_{(n) 1}\right|_{u}=\left.\mathfrak{o}_{(n) 1}\right|_{u+w_{n}}$. Thus, $\mathfrak{q}_{(n) k}$ is defined on the domain $[-(1 / c) \ln (n),(1 / c) \ln (n)] \times\left(\mathbb{R} /\left(2 \pi q_{\mathcal{E}} \mathbb{Z}\right)\right)$.

It follows from Assertion (b) of the first bullet in Lemma 7.2 that the sequence $\left\{\mathfrak{q}_{(n) 1}\right\}$ is uniformly bounded. Moreover, the sequence $\left\{\bar{\partial} \mathfrak{q}_{(n) 1}+\frac{1}{2} \mathfrak{R} \mathfrak{o}_{(n) 1}\right\}_{n=1,2, \ldots}$ converges uniformly to zero uniformly also. Lemma 7.4 implies that the sequence $\left\{\mathfrak{q}_{(n) 1}\right\}_{n=1,2, \ldots}$ has uniformly bounded Holder norm for any given exponent in $(0,1)$. As a consequence of all of this, there is a subsequence of $\left\{\mathfrak{q}_{(n) 1}\right\}_{n=1,2, \ldots}$ that converges in any such Holder topology on compact subsets of $\mathbb{R} \times\left(\mathbb{R} /\left(2 \pi q_{\mathcal{E}} \mathbb{Z}\right)\right)$ to a bounded function, $\mathfrak{q}_{1}$, that obeys the equation $\bar{\partial} \mathfrak{q}_{1}+\frac{1}{2} \mathfrak{R q}_{1}=0$. Moreover, Lemma 7.4 implies that the subsequence can be chosen so that $q_{1} \neq 0$ at some point on the $w=0$ circle. However, this is nonsense because the operator $\bar{\partial}+\frac{1}{2} \mathrm{R}$ has trivial bounded kernel.

Part 3 This part considers the case when there are two or more sets in the partition $\left\{\mathcal{E}_{1}, \ldots, \mathcal{E}_{n}\right\}$. To set the stage, suppose that $\mathcal{E} \in \mathcal{E}_{1} \cup \cdots \cup \mathcal{E}_{N}$ is not $\mathbb{R}$-invariant. Reintroduce the notation $\mathfrak{Z}_{\mathcal{E}}(u)$ for the set of $q_{\mathcal{E}}$ points in $\mathcal{E}$ 's intersection with $\{u\} \times D$. As before, each point is one of the $q_{\mathcal{E}}$ values of a multivalued function, $z_{\mathcal{E}}(u)$, that has the form $z_{\mathcal{E}}(u)=\varsigma_{\mathcal{E}} e^{-\mathrm{R} w+k_{\mathcal{E}} u / q_{\mathfrak{E}}}\left(1+\mathfrak{r}_{\mathfrak{E}}\right)$ where $\varsigma_{\mathcal{E}} \in \mathbb{C}-\{0\}$ and $\left|\mathfrak{r}_{\mathfrak{E}}\right| \leq c_{0} e^{-|w| / c_{0}}$. All ends in any given $\mathcal{E}_{b}$ have equal versions of $k_{\mathcal{E}}$ and $q_{\mathcal{E}}$. These are denoted in what follows by $k_{b}$ and $q_{b}$. The following is also true: If $\mathcal{E}$ and $\mathcal{E}^{\prime}$ are distinct elements of any given $\mathcal{E}_{b}$ then $\varsigma_{\mathcal{E}} / \varsigma_{\mathcal{E}^{\prime}}$ is not a $q_{b}$-th root of unity. If $\mathcal{E}$ is part of $\mathbb{R} \times \gamma$, then $\mathcal{E}_{N}=\{\mathcal{E}\}$. In this case set $\mathfrak{Z}_{\mathcal{E}}(u)=\{0\}$. Take $q_{\mathcal{E}}$ for such an end to be the integer that accompanies $\mathbb{R} \times \gamma$ as an element in $\vartheta$, and take $k_{\mathcal{E}}=\infty$. 
The argument now proceeds in an inductive fashion. To set up the induction, first set $\mathcal{E}_{0}=\varnothing$. Given $b \in\{1, \ldots, N\}$, say that Property $b$ is satisfied when the following is true:

Given $\varepsilon_{*}>0$, there exists $n_{*}$ such that if $n>n_{*}$ and if $(u, z) \in\left(-\infty,-w_{0}\right] \times$ $S^{1} \times D$ lies where $\alpha_{n}=0$, then one of the following is true:

- There exists $z^{\prime} \in \bigcup_{\mathcal{E} \in \mathcal{E}_{0} \cup \cdots \cup \mathcal{E}_{b}} \mathfrak{Z}_{\mathcal{E}}(u)$ with $\left|z^{\prime}-z\right|<\varepsilon_{*} \delta_{n} r_{n}^{-1 / 2}$.

- $\left|z-z^{\prime}\right|>\delta_{n} r_{n}^{-1 / 2}$ for all $z^{\prime} \in \bigcup_{\mathcal{E} \in \mathcal{E}_{1} \cup \cdots \cup \mathcal{E}_{b}} \mathfrak{Z}_{\mathcal{E}}(u)$ and $|z| \leq \varepsilon_{*} e^{\left(-\mathrm{R}+k_{b} / q_{b}\right) w}$.

The $b=0$ version of Property $b$ makes no constraints and so is tautologically satisfied. The plan for what follows is to assume Property $b-1$ for a given $b \in\{1, \ldots, N\}$ and then prove that Property $b$ holds as well. The proof that Property $b-1$ implies Property $b$ is given in the three steps that follow. Note that if Property $N$ holds, then no counterexample to the claims in Lemma 6.2 can occur on an end $\mathcal{E} \subset \bigcup_{(C, m) \in \vartheta} C$ whose constant $s$ slices converge pointwise to $\gamma$ as $s \rightarrow-\infty$.

Step 1 Assume, to the contrary, that Property $b-1$ holds but not Property $b$. This requires the existence of some $\varepsilon_{*}>0$ and a subsequence of $\left\{\left(r_{n},\left(A_{n}, \psi_{n}\right)\right)\right\}_{n=1,2, \ldots}$ with the following: For each subsequence index $n$, there is a point $\left(u_{n}=w_{n}+i t_{n}, z_{n}\right)$ where $\alpha_{n}$ is zero, and which obeys the following:

- There is no point $z^{\prime} \in \bigcup_{\mathcal{E} \in \mathcal{E}_{0} \cup \cdots \cup \mathcal{E}_{b}} \mathfrak{Z}_{\mathcal{E}}\left(u_{n}\right)$ with $\left|z^{\prime}-z_{n}\right|<\varepsilon_{*} \delta_{n} r_{n}^{-1 / 2}$.

- Either $\left|z_{n}\right|>\varepsilon_{*} e^{\left(-\mathrm{R}+k_{b} / q_{b}\right) w_{n}}$, or there exists $z^{\prime} \in \bigcup_{\mathcal{E} \in \mathcal{E}_{b}} \mathfrak{Z}_{\mathcal{E}}\left(u_{n}\right)$ with $\left|z_{n}-z^{\prime}\right| \leq \delta_{n} r_{n}^{-1 / 2}$.

Note that if $b>1$, then Property $b-1$ requires, in addition:

- $\left|z_{n}-z^{\prime}\right|>\delta_{n} r_{n}^{-1 / 2}$ for all $z^{\prime} \in \bigcup_{\mathcal{E} \in E_{1} \cup \cdots \cup E_{b-1}} \mathfrak{Z}_{\mathcal{E}}\left(u_{n}\right)$.

- Given $\varepsilon_{b}>0$, then $\left|z_{n}\right| \leq \varepsilon_{b} e^{\left(-\mathrm{R}+k_{b-1} / q_{b-1}\right) w_{n}}$ for all $n$ sufficiently large.

Various scales are involved here, these being $\left|z_{n}\right|, \delta_{n} r_{n}^{-1 / 2}$ and then the various $j \in\{1, \ldots, N\}$ versions of $e^{\left(-\mathrm{R}+k_{j} / q_{j}\right) w_{n}}$. What follows describes three consequences of (7-34) and (7-35) that say something about the relative sizes of these scales. Here is the first consequence: If $b>1$, the first item in (7-34) and the second item in (7-35) have the following consequence:

Given $\varepsilon_{b}>0$, then $\delta_{n} r_{n}^{-1 / 2} \leq \varepsilon_{b} e^{\left(-\mathrm{R}+k_{b-1} / q_{b-1}\right) w_{n}}$ for all $n$ sufficiently large. 
Indeed, the first point in (7-34) requires that $\left|z_{n}\right|+c_{0} e^{\left(-\mathrm{R}+k_{b} / q_{b}\right) w_{n}}$ be larger than $\varepsilon_{*} \delta_{n} r_{n}^{-1 / 2}$, and so the second point in (7-35) gives (7-36).

Here is the second consequence: If $b>2$, the inequality in (7-30) is consistent with the assumptions about $\left\{\delta_{n}\right\}_{n=1,2, \ldots}$ only if

$$
r_{n}^{-1 / 2+1 / c_{0}}<e^{\left(-\mathrm{R}+k_{b-2} / q_{b-2}\right) w_{n}}
$$

when $n$ is large. Indeed, were the opposite inequality to hold for a large $c_{0}$, then $e^{\left(-\mathrm{R}+k_{b-1} / q_{b-1}\right) w_{n}}$ and hence $\delta_{n} r_{n}^{-1 / 2}$ would be less than $r_{n}^{-1 / 2-1 / c_{0}}$.

Here is the final consequence: What with the definition of $\delta_{n}$, the first item in (7-35) implies the following:

$$
\text { There exists } z^{\prime} \in \bigcup_{\mathcal{E} \in \mathcal{E}_{b} \cup \cdots \cup \mathcal{E}_{N}} \text { such that }\left|z_{n}-z^{\prime}\right| \leq \delta_{n} r_{n}^{-1 / 2} .
$$

This is true whether $b=1$ or $b>1$.

The subsequent discussion considers separately two possibilities that correspond to the dichotomy that is depicted in (7-25). These are as follows:

- There exists $\varepsilon>0$ and a subsequence of $\left\{\left(r_{n},\left(A_{n}, \psi_{n}\right)\right)\right\}_{n=1,2, \ldots}$ with a corresponding $\left(u_{n}, z_{n}\right) \in \alpha_{n}^{-1}(0)$ such that (7-34) holds with $\varepsilon e^{\left(-\mathrm{R}+k_{b} / q_{b}\right) w_{n}}<\delta_{n} r_{n}^{-1 / 2}$.

- Fix any $\varepsilon>0$. If $n$ is sufficiently large, then any point $\left(u_{n}, z_{n}\right) \in \alpha_{n}^{-1}(0)$ that obeys (7-34) is such that $\varepsilon e^{\left(-\mathrm{R}+k_{b} / q_{b}\right) w_{n}}>\delta_{n} r_{n}^{-1 / 2}$.

To derive nonsense in either case, pass to a subsequence of $\left\{\left(r_{n},\left(A_{n}, \psi_{n}\right)\right)\right\}_{n=1,2, \ldots}$ (hence renumbered consecutively from 1) where (7-32) holds and where $\left\{\delta_{n}\right\}_{n=1,2, \ldots}$ either converges or else increases with no upper bound. What with (7-33), the subsequence can and should be chosen when $b>1$ so that $\delta_{n} r_{n}^{-1 / 2} \leq n^{-4} e^{\left(-\mathrm{R}+k_{b-1} / q_{b-1}\right) w_{n}}$. Additional conditions on this subsequence will be imposed below.

Step 2 This step derives nonsense when the top item in (7-39) is relevant. In this case, no generality is lost by assuming that the top item holds for a given $\varepsilon$ and for all indices $n$, and that the sequence $\left\{\left(16 \delta_{n} r_{n}^{-1 / 2}\right)^{-1} e^{\left(-\mathrm{R}+k_{b} / q_{b}\right) w_{n}}\right\}_{n=1,2, \ldots}$ converges.

Let $m=\sum_{\mathcal{E} \in \mathcal{E}_{b} \cup \cdots \cup \mathcal{E}_{N}} q_{\mathcal{E}}$ and construct for each $n$ and for each $k \in\{1, \ldots, m\}$, a $\mathbb{C}-$ valued function $\mathfrak{o}_{(n) k}$ on $\left[w_{n}-\left(1 /\left(50 \mathrm{~m}^{2}\right)\right) \ln (n), w_{n}+\left(1 /\left(50 \mathrm{~m}^{2}\right)\right) \ln (n)\right] \times S^{1}$ as done in Lemma 7.2 using the following data: Take $C$ to be the $\left[w_{n}-\left(1 /\left(50 \mathrm{~m}^{2}\right)\right) \ln (n)\right.$, $\left.w_{n}+\left(1 /\left(50 \mathrm{~m}^{2}\right)\right) \ln (n)\right] \times S^{1}$ portion of $\mathbb{R} \times \gamma$. Meanwhile, define $\hat{A}$ using the function $\wp$ given by $\wp(x)=x \chi(x)+1-\chi(x)$. Finally, take $\rho=\rho_{n}=100 \delta_{n} r_{n}^{-1 / 2}$, 
take $z=z_{n}=n \delta_{n} r_{n}^{-1 / 2}$, and take $\rho_{\diamond n}$ to be $16 \delta_{n} r_{n}^{-1 / 2}$. Define the function $\mathfrak{q}_{(n) k}$ on $\left[w_{n}-\left(1 /\left(50 \mathrm{~m}^{2}\right)\right) \ln (n), w_{n}+\left(1 /\left(50 \mathrm{~m}^{2}\right)\right) \ln (n)\right] \times S^{1}$ by $\left.\mathfrak{q}_{(n) k}\right|_{u}=\left.\mathfrak{o}_{(n) k}\right|_{u+w_{n}}$.

The next lemma plays a principal role in what follows.

Lemma 7.8 There exists $\kappa_{*} \geq 50 \mathrm{~m}^{2}$ with the following significance: Take $n \geq \kappa$. Then all points $(u, z) \in \alpha_{n}^{-1}(0)$ with $u \in\left[w_{n}-\left(1 / \kappa_{*}\right) \ln (n), w_{n}+\left(1 / \kappa_{*}\right) \ln (n)\right] \times S^{1}$ and with $|z| \leq z_{n}$ obey

$$
|z| \leq \kappa_{*}\left(\delta_{n} r_{n}^{-1 / 2}+e^{\left(-\mathrm{R}+k_{b} / q_{b}\right) w}\right) .
$$

Moreover, $\left|\alpha_{n}\right|^{2} \geq \frac{1}{2}$ at all points $(u, z)$ with $u$ as above and with $z$ such that $|z| \epsilon$ $\left[\frac{1}{4} z_{n}, z_{n}\right]$.

Proof of Lemma 7.8 Suppose that $c>1$ and that $u \in\left[w_{n}-\ln (n), w_{n}+(1 / c) \ln (n)\right] \times$ $S^{1}$ and that $z \in D$ are such that $\alpha(u, z)=0$ and $|z| \leq 100 z=100 n \delta_{n} r_{n}^{-1 / 2}$. Given that $c>c_{0}$, and given the fact that $\delta_{n} r_{n}^{-1 / 2} \leq n^{-4} e^{\left(-\mathrm{R}+k_{b-1} / q_{b-1}\right) w_{n}}$, this implies that

$$
\left|z-z^{\prime}\right| \geq 2 \delta_{n} r_{n}^{-1 / 2} \quad \text { for all } z^{\prime} \in \bigcup_{\mathcal{E} \in \mathcal{E}_{1} \cup \cdots \cup \mathcal{E}_{b-1}} \mathfrak{Z}_{\mathcal{E}}(u)
$$

when $n$ is large. Thus, there exists $z^{\prime} \in \bigcup_{\mathcal{E} \in \mathcal{E}_{b} \cup \ldots \cup \mathcal{E}_{N}} \mathfrak{Z}_{\mathcal{E}}(u)$ such that $\left|z-z^{\prime}\right| \leq$ $\delta_{n} r_{n}^{-1 / 2}$. This implies that

$$
|z| \leq \delta_{n} r_{n}^{-1 / 2}+c_{0} e^{\left(-\mathrm{R}+k_{b} / q_{b}\right) w} .
$$

To obtain the final assertion of the lemma, remark that this last bound is less than $\delta_{n} r_{n}^{-1 / 2}+n^{1 / 2} e^{\left(-\mathrm{R}+k_{b} / q_{b}\right) w_{n}}$ if $c>c_{0}$. If $n$ is large, this upper bound requires that $|z| \leq n^{-1 / 4} z_{n}$.

Let $\kappa_{*}$ be as given in Lemma 7.8. Here is a first consequence of Lemma 7.8. If $u \in\left[-\left(1 / \kappa_{*}\right) \ln (n),\left(1 / \kappa_{*}\right) \ln (n)\right] \times S^{1}$, then

$$
\left|\mathfrak{q}_{(n) k}\right| \leq c_{0}\left(1+e^{\left(-\mathrm{R}+k_{b} / q_{b}\right) w}\right)^{k} .
$$

Indeed, this follows from the second bullet of Lemma 7.1. What follows is a second consequence of Lemma 7.8. This one follows with the help of the second bullet in Lemma 7.2. If $u \in\left[-\left(1 / \kappa_{*}\right) \ln (n),\left(1 / \kappa_{*}\right) \ln (n)\right] \times S^{1}$, then

$$
\bar{\partial} \mathfrak{q}_{(n) k}+\frac{1}{2} k \operatorname{Rq}_{(n) k}=\mathfrak{e}_{(n)},
$$

where $\left\{\left|e_{(n)}\right|\right\}_{n=1,2, \ldots}$ is a sequence with limit zero.

It follows from (7-41) and (7-42), just as in the proof of Lemma 7.3, that each $k \in$ $\{1, \ldots, q\}$ version of the sequence $\left\{\mathfrak{q}_{(n) k}\right\}_{n=1,2, \ldots}$ has a subsequence that converges 
uniformly on compact subsets of $\mathbb{R} \times S^{1}$ in the Holder topology for any given exponent in $(0,1)$. Denote the limit by $\mathfrak{q}_{k}$. This limit obeys

$$
\left|\mathfrak{q}_{k}\right| \leq c_{0}\left(1+e^{-\left(-\mathrm{R}+k_{\mathfrak{E}} / q\right) w}\right)^{k} \quad \text { and } \quad \bar{\partial} \mathfrak{q}_{(n) k}+\frac{1}{2} k \mathrm{R} \mathfrak{q}_{(n) k}=0 .
$$

Write $q=h q_{b}+m_{b}$ where $m_{b} \in\left\{0, \ldots, q_{b}-1\right\}$, and let $c$ denote the limit of the sequence

$$
\left\{\left(16 \delta_{n} r_{n}^{-1 / 2}\right)^{-1} e^{\left(-\mathrm{R}+k_{\mathcal{E}} / q_{\mathcal{E}}\right) w_{n}}\right\}_{n=1,2, \ldots} .
$$

As explained next, these $q$ different subsequences can be chosen so as to ensure that (7-29) holds. To see why this must be true, note first that the assumption in the first item of (7-39) requires that

$$
\left(\delta_{n} r_{n}^{-1 / 2}\right)^{-1} e^{\left(-\mathrm{R}+k_{b^{\prime}} / q_{b^{\prime}}\right) w_{n}} \leq c_{0}\left(\frac{1}{n}\right)^{1 / c_{0}}
$$

if $b^{\prime}>b$. These assumptions have the following additional implication: Recall that any given end $\mathcal{E}$ is the graph of a multivalued function

$$
u \rightarrow z_{\mathcal{E}}(u)=\varsigma_{\mathcal{E}} e^{-\mathrm{R} w+k_{\mathfrak{E}} u / q_{\mathcal{E}}}\left(1+\mathfrak{r}_{\mathfrak{E}}\right) .
$$

Then $\left|\mathfrak{r}_{\mathcal{E}}\right|$ is also bounded above by $c_{0}\left(\frac{1}{n}\right)^{1 / c_{0}}$. These bounds with (7-34) guarantee the existence of subsequences with limits $\left\{\mathfrak{q}_{k}\right\}_{1 \leq k \leq q}$ that obey (7-29).

Just as in Step 2 of Part 2, the fact that (7-29) holds leads to nonsense. Indeed, define the $u$-dependent polynomials, monic degree $m$ polynomials $\mathcal{P}(\lambda)$ and $\mathcal{P}_{0}(\lambda)$ using the following analog of (7-30):

$$
\begin{array}{ll}
\text { - } & \left.\mathcal{P}(\lambda)\right|_{u}=\lambda^{m}+\sum_{1 \leq k \leq m} \mathfrak{q}_{k}(u) \lambda^{m-k}, \\
\text { - } & \mathcal{P}_{0}(\lambda)=\lambda^{m}+\sum_{1 \leq x \leq h} \mathfrak{c}^{x q_{\mathcal{E}}}\left(\sum_{\mathcal{E} \in \mathcal{E}_{b}} \varsigma_{\mathcal{E}}^{x q_{\mathcal{E}}}\right) e^{-x\left(q_{\mathcal{E}} \mathrm{R} w+k_{\mathcal{E}} u\right)} \lambda^{m-x q_{\mathcal{E}}},
\end{array}
$$

where $h$ here is the number of ends that comprise $\mathcal{E}_{b}$. What is asserted by (7-31) holds here for the same reason that it holds in Step 2 of Part 2. As in Step 2 of Part 2, the assertion in (7-31) and the fact that the sequences $\left\{\left\{\mathfrak{q}_{(n) k}\right\}_{n=1,2, \ldots}\right\}_{1 \leq k \leq m}$ converge pointwise on bounded domains to $\left\{\mathfrak{q}_{k}\right\}_{1 \leq k \leq m}$ demands, for all large $n$, a point $(u, z) \in \alpha_{n}^{-1}(0)$ such that

$$
\left|z-z^{\prime}\right|>16 \Delta \delta_{n} r_{n}^{-1 / 2} \text { for all } z^{\prime} \in \bigcup_{\mathcal{E} \in \mathcal{E}_{b} \cup \cdots \cup \mathcal{E}_{N}} \mathfrak{Z}_{\mathcal{E}}(u)
$$

when $\Delta>c_{0}$. Of course, this is nonsense because any $(u, z) \in \alpha_{n}^{-1}(0)$ must have distance $\delta_{n} r_{n}^{-1 / 2}$ or less from $\bigcup_{\mathcal{E} \in \mathcal{E}_{1} \cup \cdots \cup \mathcal{E}_{N}} \mathcal{E} \in \mathcal{E}_{1}$.

Step 3 This step derives nonsense when the second item in (7-39) is assumed. The derivation of nonsense for this case is very nearly the same as that done in Step 3 of Part 2. To say a bit more, remark first that no generality is lost by choosing the 
subsequence of $\left\{\left(r_{n},\left(A_{n}, \psi_{n}\right)\right)\right\}_{n=1,2, \ldots}$ and then renumbering consecutively from 1 so that if (7-34) holds for any given $\left(u_{n}, z_{n}\right) \in \alpha_{n}^{-1}(0)$, then

$$
e^{\left(-\mathrm{R}+k_{b} / q_{b}\right) w_{n}} \geq e^{n} \delta_{n} r_{n}^{-1 / 2} .
$$

Granted this last condition, the second bullet in (7-34) and (7-38) demand a point $z^{\prime} \in \bigcup_{\mathcal{E} \in \mathcal{E}_{b}} \mathfrak{Z}_{\mathcal{E}}\left(u_{n}\right)$ such that $\left|z_{n}-z^{\prime}\right| \leq \delta_{n} r_{n}^{-1 / 2}$.

Fix an increasing, unbounded sequence $\left\{\chi_{n}\right\}_{n=1,2}, \ldots \subset[8, \infty)$ but such that the sequence $\left\{\chi_{n}^{2} \delta_{n} r_{n}^{-1 / 2}\right\}$ converges to zero. Choose $x_{n} \leq n$. One additional upper bound on the growth of the sequence $\left\{\chi_{n}\right\}_{n=1,2, \ldots}$ arises below. Set $\rho_{n}=\chi_{n} \delta_{n} r_{n}^{-1 / 2}$ and set $z_{n}=n^{2} \delta_{n} r_{n}^{-1 / 2}$. This choice for $\left\{z_{n}\right\}_{n=1,2, \ldots}$ guarantees the existence of a constant $c \geq 1$ with the following property:

Let $\Sigma_{n}$ denote the $w \in\left[w_{n}-(1 / c) \ln (n), w_{n}+(1 / c) \ln (n)\right]$ part of $\bigcup_{\mathcal{E} \in \mathcal{E}_{b}} \mathcal{E}$. If $n$ is large, then the exponential map embeds the radius $2 z_{n}$ disk bundle in $N_{1} \mid \Sigma_{n}$.

With $\rho_{n}$ and $z_{n}$ understood, set $\rho_{\diamond n}=16 \delta_{n} r_{n}^{-1 / 2}$. Now repeat verbatim what is said in the final three paragraphs of Step 3 in Part 2 to obtain nonsense.

\section{Perturbations of (1-11)}

This last section proves the assertions that are made by Item (iii) from the first bullet of [8, Theorem 4.3]. The first subsection below reviews some of the notation and restates the claim as Proposition 8.1. The second subsection gives a proof.

\section{8.a Perturbations}

As noted in [8, Sections 1.c, 1.d], it is often necessary to modify (1-11) so as to guarantee that all instanton solutions with nondegenerate $s \rightarrow \pm \infty$ limits are suitably nondegenerate in their own right. An allowed perturbation is defined by the choice of a gauge invariant function on $\operatorname{Conn}(E) \times \mathbb{C}^{\infty}(M ; \mathbb{S})$. Such a function must come from a certain Banach space of smooth functions. This space is described in the aforementioned subsections of [8]. It is denoted in [8] and here by $\mathcal{P}$. Let $\mathfrak{p}$ denote a given element in $\mathcal{P}$. The resulting version of (1-8) can be viewed as the equations that define the critical points of the function $\mathfrak{a}+\mathfrak{p}$ on $\operatorname{Conn}(E) \times \mathbb{C}^{\infty}(M ; \mathbb{S})$ with a given by (3-2). The resulting perturbed version of (1-8) is written schematically as

$$
\begin{array}{ll}
\text { - } & B_{A}-r\left(\psi^{\dagger} \tau \psi-i a\right)-i * d \mu+\left.\mathfrak{T}\right|_{(A, \psi)}+\frac{1}{2} B_{A_{K}}=0 . \\
\text { - } & D_{A} \psi-\left.\mathfrak{S}\right|_{(A, \psi)}=0 .
\end{array}
$$


The corresponding version of (1-11) has the form

$$
\begin{aligned}
& \text { - } \frac{\partial}{\partial s} A+B_{A}-r\left(\psi^{\dagger} \tau \psi-i a\right)-i * d \mu-\left.\mathfrak{T}\right|_{(A, \psi)}+\frac{1}{2} B_{A_{K}}=0 . \\
& \text { - } \frac{\partial}{\partial s} \psi+D_{A} \psi-\left.\mathfrak{S}\right|_{(A, \psi)}=0 .
\end{aligned}
$$

A solution to (8-2) is said to be an instanton if its $s \rightarrow \pm \infty$ limits are solutions to (8-1).

There is a corresponding version of the symmetric operator that is depicted in (1-14), this given in $[8,(3-7)]$. There is, likewise, a corresponding version of the operator in (1-12), this depicted by [8, (3-9)]. A solution to (8-1) is said to be nondegenerate when the latter's version of $[8,(3-7)]$. Let $\mathfrak{d}$ denote an instanton solution to (8-2) with nondegenerate $s \rightarrow \pm \infty$ limits. Then $\mathfrak{d}$ 's version of [8, (3-9)] defines a Fredholm operator in a suitable sense. The instanton $\mathfrak{d}$ is said to be nondegenerate when the latter operator has trivial cokernel.

Section 3.b introduced the notion of the spectral flow, $f_{\mathfrak{d}}$, along an instanton solution to (1-11) with nondegenerate $s \rightarrow \pm \infty$ limits. This notion is well defined for the instanton solutions to (8-2) with nondegenerate $s \rightarrow \pm \infty$ limits if it is understood that the term spectral flow in this context refers to the following family of operators: Let $\mathfrak{d}$ denote the given instanton. The corresponding family of operators is parametrized by $\mathbb{R}$. The $s \in \mathbb{R}$ member is the version of the operator that is defined in [8, (3-7)] using the connection and spinor from $\left.\mathfrak{d}\right|_{s}$.

Given a pair of nondegenerate solutions $\mathfrak{c}_{-}, \mathfrak{c}_{+}$to $(8-1)$, use $\mathcal{M}_{1, p}\left(\mathfrak{c}_{-}, \mathfrak{c}_{+}\right)$to denote the space of instanton solutions to (8-2) with $s \rightarrow-\infty$ equal to $\mathfrak{c}_{-}$, with $s \rightarrow \infty$ limit gauge equivalent to $\mathfrak{c}_{+}$, and with spectral flow function $f_{\mathfrak{d}}=1$. If all instantons in $\mathcal{M}_{1, p}\left(\mathfrak{c}_{-}, \mathfrak{c}_{+}\right)$are nondegenerate, then the latter space is a smooth manifold of dimension 1 with a free $\mathbb{R}$ action that is induced by the constant translations along the $\mathbb{R}$ factor of $\mathbb{R} \times M$.

The upcoming Proposition 8.1 restates Item (iii) from the first bullet of [8, Theorem 4.2]. This proposition refers to a pair $(a, J)$, of contact form in $\mathcal{N}_{M}$ and almost complex structure from $g_{a}$. Given $L \geq 1$, this pair is assumed to obey (1-15). The set $Z_{\text {ech }}^{L}$ is defined by $(a, J)$. The proposition specifies a constant $\kappa$; it is implicit that Theorem 1.1 and Theorem 1.2 can be invoked using $L$ and $(a, J)$ when $r>\kappa$.

Proposition 8.1 Fix $L \geq 1$ and a pair $(a, J)$ just described. There exists $\kappa \geq 1$ with the following significance: Define the space $\mathcal{M}^{r}$ using the pair $(a, J)$, a $1-$ form $\mu \in \Omega$ with $\mathcal{P}$-norm bounded by 1 , and $r \geq \kappa$. Let $\Theta_{-}$and $\Theta_{+}$denote any two elements in $Z_{\text {ech. }}^{L}$. Use $\mathfrak{c}_{-}$and $\mathfrak{c}_{+}$to denote solutions to (1-8) whose gauge equivalences classes are the respective images in $\mathcal{M}^{r}$ of $\Theta_{-}$and $\Theta_{+}$via Theorem 4.2's map $\Phi^{r}$. Let $\mathfrak{p} \in \mathcal{P}$ 
denote a sufficiently small element that vanishes to second order on the image of $\Phi^{r}$. Define $\mathcal{M}_{1, \mathfrak{p}}\left(\mathfrak{c}_{-}, \mathfrak{c}_{+}\right)$as above. There exists an $\mathbb{R}$-equivariant diffeomorphism from $\mathcal{M}_{1}\left(\Theta_{-}, \Theta_{+}\right)$to $\mathcal{M}_{1, \mathfrak{p}}\left(\mathfrak{c}_{-}, \mathfrak{c}_{+}\right)$.

\section{8.b Proof of Proposition 8.1}

The proof has four parts.

Part 1 Let $r_{L}>1$ be such that Items (i) and (ii) of the first bullet in [8, Theorem 4.3 ] holds for $r \geq r_{L}$ and fix $r \geq r_{L}$. If $\mathfrak{p}$ is sufficiently small, perturbation theory finds an $\mathbb{R}$-equivariant embedding $\Xi_{\mathfrak{p}}: \mathcal{M}_{1}\left(\mathfrak{c}_{-}, \mathfrak{c}_{+}\right) \rightarrow \mathcal{M}_{1, \mathfrak{p}}\left(\mathfrak{c}_{-}, \mathfrak{c}_{+}\right)$onto a union of smooth components of $\mathcal{M}_{1, \mathfrak{p}}$. This map varies with $\mathfrak{p}$ in a suitably smooth manner, and is such that $\Xi_{0}$ is the identity. In particular, if $\mathfrak{d} \in \mathcal{M}_{1}\left(\mathfrak{c}_{-}, \mathfrak{c}_{+}\right)$and $\left\{\mathfrak{p}_{k}\right\}_{k=1,2, \ldots}$ is a sequence in $\mathcal{P}$ that converges to zero, then the corresponding sequence $\left\{\Xi_{\mathfrak{p}_{k}}(\mathfrak{d})\right\}$ converges to $\mathfrak{d}$ in the topologies that are considered in Chapter 16 of [5]. For example, this sequence converges to zero in any given $n \geq 0$ version of $C^{n}$ topology on maps from $\mathbb{R}$ to the space $\operatorname{Conn}(E) \times \mathbb{C}^{\infty}(M ; \mathbb{S})$. Granted the preceding, Proposition 8.1 follows if it is the case that $\Xi_{\mathfrak{p}}$ is surjective when ever $\mathfrak{p}$ has suitably small norm. The parts that follow prove that this is the case by deriving a contradiction if it is not.

Part 2 Assume that there exists an increasing, unbounded sequence $\left\{r_{i}\right\}_{i=1,2, \ldots} \subset$ $\left[r_{L}, \infty\right)$ and a corresponding sequence $\left\{\mathfrak{p}_{i, k}\right\}_{k=1,2, \ldots} \subset P$ that converges to zero with the following property: Fix any given index pair $(i, k)$ and there is an instanton, $\mathfrak{d}_{i, k}$, in the $\mathfrak{p}=\mathfrak{p}_{i, k}$ version of $\mathcal{M}_{1, p}\left(\mathfrak{c}_{-}, \mathfrak{c}_{+}\right)$that is not in the image of the corresponding version of $\Xi_{\mathfrak{p}}$.

To derive nonsense from this assumption fix $i$ and let $r=r_{i}$. The lemma that follows plays a key role in the subsequent argument.

Lemma 8.2 There exists $\kappa \geq 1$ such that if $r=r_{i} \geq \kappa$, then the following is true: For each $k \in\{1,2, \ldots\}$ write the $s \rightarrow \infty$ limit of $\mathfrak{d}_{i, k}$ as $u_{i, k} \mathfrak{c}_{+}$. Then $\mathfrak{a}\left(\mathfrak{c}_{-}\right)-\mathfrak{a}\left(u_{i, k} \mathfrak{c}_{+}\right) \leq$ $2 \pi L r$.

Proof of Lemma 8.2 Consider first the case when $c_{1}(\operatorname{det}(\mathbb{S}))$ is a torsion class. If this is so, then $\mathfrak{a}\left(u_{i, k} \mathfrak{c}_{+}\right)=\mathfrak{a}\left(\mathfrak{c}_{+}\right)$. Meanwhile, both $\mathfrak{c}_{-}$and $\mathfrak{c}_{+}$define gauge equivalence classes in the image of $\Phi^{r}$. As such, both have $\mathrm{E}<2 \pi L$. It follows from this and (5-19) that both are such that $|\mathfrak{a}|<\pi L r$ if $r$ is large. Thus, $\mathfrak{a}\left(\mathfrak{c}_{-}\right)-\mathfrak{a}\left(u_{i, k} \mathfrak{c}_{+}\right)<2 \pi L r$ if $r$ is large.

Suppose instead that $c_{1}(\operatorname{det}(\mathbb{S}))$ is not a torsion class. It follows from the fourth bullet in (8-3) that $\left|\mathfrak{a}\left(\mathfrak{c}_{-}\right)-\mathfrak{a}\left(u_{i, k} \mathfrak{c}_{+}\right)\right| \leq\left|\mathfrak{a}^{f}\left(\mathfrak{c}_{-}\right)-\mathfrak{a}^{f}\left(u_{1, k} \mathfrak{c}_{+}\right)\right|+2 \pi^{2}$. Meanwhile, as $\mathfrak{c}_{-}$ and $u_{i, k} \mathfrak{c}_{+}$are such that $\mathrm{E}<2 \pi L$, it follows from (5-25) both are such that $\left|\mathfrak{a}^{f}\right|<\pi L r$ when $r$ is large. These last two inequalities prove the claim. 
Part 3 Given Lemma 8.2, it follows from the analysis in Chapters 16-19 of [5] that there is a subsequence of this sequence (hence renumbered consecutively from 1) that converges to what is called a broken trajectory. A broken trajectory in this case consists of a finite, ordered set of instanton solutions to (1-11). Let $N=N_{i}$ denote the number of elements in this set, and use $\left\{\mathfrak{d}^{j}\right\}_{1 \leq j \leq N}$ to denote the set itself with the dependence on the index $i$ understood. To say more about this set, use $\mathfrak{c}_{+}^{j}$ to denote the $s \rightarrow \infty$ limit of $\mathfrak{d}^{j}$ and use $\mathfrak{c}_{-}^{j}$ to denote the $s \rightarrow-\infty$ limit $\mathfrak{d}^{j}$. Then

- $\mathfrak{c}_{-}^{j}=\mathfrak{c}_{+}^{j-1}$ for each $j \in\{2, \ldots, N\}$.

- $\mathfrak{c}_{-}^{1}=\mathfrak{c}_{-}$and $\mathfrak{c}_{+}^{N}=u \mathfrak{c}_{+}$for some $\left.u \in C^{\infty}\left(M ; S^{1}\right)\right)$.

- $\mathfrak{a}\left(\mathfrak{c}_{-}^{1}\right)>\mathfrak{a}\left(\mathfrak{c}_{+}^{j}\right)$ for each $j \in\{1, \ldots, N\}$.

- $\sum_{1 \leq j \leq N} f_{\mathfrak{d}_{j}}=1$.

Note that $\left\{\mathfrak{d}_{i, k}\right\}_{k=1,2, \ldots}$ must stay uniformly far in any given $C^{n}$ norm from any instanton solution to (1-11) from $\mathcal{M}_{1}\left(\mathfrak{c}_{-}, \mathfrak{c}_{+}\right)$for if not, then any given large $k$ version would be in the image of the corresponding version of $\Xi_{\mathfrak{p}}$. This last fact about $\left\{\mathfrak{d}_{i, k}\right\}_{1,2, \ldots}$ implies that $N_{i}>1$. A proof that $N_{i}=1$ for sufficiently large $i$ generates the desired nonsense.

Part 4 It follows from the first bullet in (8-3) that any given sufficiently large index $i$ version of the sequence $\left\{\mathfrak{a}\left(\mathfrak{c}_{-}^{j}\right)\right\}_{1 \leq j \leq N}$ is such that

$$
\mathfrak{a}\left(\mathfrak{c}_{-}\right)=\mathfrak{a}\left(\mathfrak{c}_{-}^{1}\right)>\cdots>\mathfrak{a}\left(\mathfrak{c}_{-}^{N}\right)=\mathfrak{a}\left(u \mathfrak{c}_{+}\right) .
$$

This chain of inequalities implies that $\mathfrak{a}\left(\mathfrak{c}_{-}^{j}\right)-\mathfrak{a}\left(\mathfrak{c}_{+}^{j}\right) \leq 2 \pi L r_{i}$ for each $j \in\{1, \ldots, N\}$ when the index $i$ is large. This is to say that each $\mathfrak{d} \in\left\{\mathfrak{d}^{j}\right\}_{1 \leq j \leq N}$ version of what is denoted in Proposition 5.1 by $A_{\mathfrak{d}}$ is bounded by $2 \pi L r_{i}$. It follows from this using Proposition 5.5 that each $\mathfrak{c}_{-}^{j}$ is such that $\mathrm{E}\left(\mathfrak{c}_{-}^{j}\right)$ is bounded by $2 \pi L$ when $r=r_{i}>c_{0}$. Thus, each is in the image of $\Phi^{r}$. Proposition 5.5 implies even more: There is a broken $J$-holomorphic trajectory of the sort described in the proof of Lemma 6.1 that interpolates between $\Theta_{-}$and $\Theta_{+}$. Denote the constituents as $\left\{\vartheta_{k}\right\}_{1 \leq k \leq N^{\prime}}$. An argument that differs little from that used in Part 2 of the proof of Lemma 6.1 proves that their embedded contact homology indices must add to 1 . This being the case, if $N=N_{i}>1$, then at least one of the broken $J$-holomorphic trajectories must contain an element $\vartheta$ that has nonpositive embedded contact homology index. As each contains a non- $\mathbb{R}$-invariant subvariety, this event is not possible given the assumed genericity of the pair $(a, J)$. 


\section{Index to the notation}

$$
\begin{aligned}
& \phi^{r} \\
& \psi^{r}
\end{aligned}
$$

$a$ : the contact $1-$ form

$\Gamma$ : a class in $H_{1}(M ; \mathbb{Z})$

$k^{-1}$ : the kernel of $a$ oriented by $-d a$

$c_{1}(k)$ : the first Chern class of $k$

$v$ : the Reeb vector field

Reeb orbit: a closed integral curve of $v$, typically denoted by $\gamma$

$\Theta$ : a finite set of pairs $(\gamma, m)$ with $\gamma$ a Reeb Part 1 of Section 1.a orbit and $m$ a positive integer

$\mathcal{Z}$ : a set of $\Theta$ 's as above satisfying particular Part 1 of Section 1.a constraints

$\ell_{\gamma}$ : when $\gamma$ is a Reeb orbit, the integral of $a$ Part 1 of Section 1.a along $\gamma$

$\mathcal{Z}^{L}$ : a subset of $\mathcal{Z}$ with a length bound given Part 1 of Section 1.a by $L$ on the Reeb orbits

$J$ : the given almost complex structure

$\gamma$ : a Reeb orbit, aka a closed integral curve of $v$

$(v, \mu)$

$\mathcal{L}$ : a differential operator associated to a Reeb orbit

nondegenerate

elliptic, hyperbolic

rotation number

$\mathrm{R}$ : the rotation number for an elliptic Reeb orbit

$L$-nondegenerate

$\mathfrak{C}_{m}$ : vortex moduli space

f

$\nabla^{(1,0)} \hat{h}$

nondegenerate in the context of a map c: $S^{1} \rightarrow$ $\mathfrak{C}_{m}$

$\mathfrak{C} \Theta$

$\mathfrak{C} \Theta^{*}$

Part 1 of Section 1.a
Equation (1-10), Theorem 4.2 of [8]

Equation (1-16), Theorem 4.3 of [8]

Part 1 of Section 1.a

Part 1 of Section 1.a

Part 1 of Section 1.a

Part 1 of Section 1.a

Part 1 of Section 1.a

Part 2 of Section 1.a

defined for a given Reeb orbit in Equation (1-1)

Equation (1-2)

Part 2 of Section 1.a

Part 2 of Section 1.a

Part 2 of Section 1.a

Part 2 of Section 1.a

Part 2 of Section 1.a

Part 3 of Section 1.a

Equation (1-5)

Part 3 of Section 1.a

Part 3 of Section 1.a

Part 3 of Section 1.a

Part 3 of Section 1.a 
metric on TM

S: spinor bundle

Conn $(E)$

$B_{A}$

$\psi^{\dagger} \tau \psi$

$D_{A}$

$\mu$

$A_{K}$

$\operatorname{cl}(\cdot)$ : Clifford multiplication map

$\Omega$

$\mathcal{P}$

$\mathcal{P}$-norm

$\mathcal{M}^{r}$

$\mathrm{E}(A)$

$\mathfrak{C Z}$

$\mathfrak{C} \mathcal{Z}^{L *}$

$\mathcal{N}_{M}$

$g_{a}$

$\mathcal{Z}_{\text {ech }}$

$\mathcal{M}_{1}\left(\Theta_{-}, \Theta_{+}\right)$

$I\left(\Theta_{-}, \Theta_{+}, Z\right)$

$\mathfrak{d}$

instanton

$\mathfrak{D}_{\mathfrak{d}}$

$\mathbb{H}$

$\mathbb{L}$

$\|\cdot\|_{\mathbb{H}}$

$\mathfrak{L}_{\mathfrak{c}}$

nondegenerate in the context of a solution to

Equation (1-8)

nondegenerate in the context of a solution to Equation (1-11)

$\mathcal{M}_{1}\left(\mathfrak{c}_{-}, \mathfrak{c}_{+}\right)$: an instanton moduli space

$\mathcal{Z}_{\text {ech }}^{L}$

$\mathcal{Z}_{\text {ech }}^{L *}$
Part 4 of Section 1.a

Part 4 of Section 1.a

Part 4 of Section 1.a

Part 4 of Section 1.a

Part 4 of Section 1.a

Part 4 of Section 1.a

Part 4 of Section 1.a

Part 4 of Section 1.a

Part 4 of Section 1.a

Part 4 of Section 1.a

Part 4 of Section 1.a

Part 4 of Section 1.a

Part 4 of Section 1.a

Equation (1-9)

Part 4 of Section 1.a

Part 4 of Section 1.a

Part 1 of Section 1.b

Part 1 of Section 1.b

Part 1 of Section 1.b

Part 1 of Section 1.b

Part 1 of Section 1.b and Equation (2-9) of [8], Section 2.c of [8]

a solution to (1-11)

Part 2 of Section 1.b

the operator in Equation (1-12)

Part 2 of Section 1.b

Part 2 of Section 1.b

Equation (1-13)

the operator in Equation (1-14)

Part 2 of Section 1.b

Part 2 of Section 1.b

Part 2 of Section 1.b

Part 3 of Section 1.b

Part 3 of Section 1.b 
a constant that is greater than 1 and is independent of what ever relevant data is under consideration. The precise value of $c_{0}$ can increase between subsequent appearances.

$\chi$ : a smooth function from $R$ to $[0,1]$ that equals 1 on $\left(-\infty, \frac{5}{16}\right]$ and value 0 on $\left[\frac{7}{16}, \infty\right)$ $\alpha, \beta$ : respective $E$ and $E K^{-1}$ components of a Part 1 of Section 2.a spinor $\psi$

$\nabla_{A}$

$\vartheta_{\mathrm{c}}$

$\left.\Pi\right|_{t}$

$\widehat{r}_{\gamma}$

$\vartheta_{\mathfrak{c *}}^{\dagger}$

$\left(A^{*}, \psi^{*}\right)$ in the context of the proof of Theorem 1.1

$\left\{\left(\mathfrak{c}_{\gamma}, \zeta_{\gamma}\right)\right\}_{(\gamma, m) \in \Theta}$

$\left\{U_{\gamma}\right\}(\gamma, m) \in \Theta$ and $U_{0}$

admissible extension

$\theta$ : product connection

$\mathfrak{J}=\left\{\left(\mathfrak{c}_{\gamma}, \zeta_{\gamma}\right)\right\}_{(\gamma, m) \in \Theta}$

$w: w=1-|\alpha|^{2}$

$f_{\mathfrak{d}}$ : spectral flow along the path $d$

$\mathrm{F}_{\mathfrak{d}}$

$\mathfrak{a}$

$\mathfrak{c s}$

$\mathfrak{e}_{\mu}$

$\mathrm{A}_{\mathfrak{d}}$

$B_{(A, \psi))}$

$p(\cdot)$

$\underline{\mathrm{M}}(\cdot)$

$X_{*}$

$\widehat{A}$

$F \hat{A}$
Part 1 of Section 2.a

Equation (2-8)

Property 3 in Part 4 of Section 2.a

Part 3 of Section 2.a

Part 4 of Section 2.a

Part 5 of Section 2.a

Part 5 of Section 2.a, Step 4 in Section 3.a of [9]

Step 1 of Part 5 of Section 2.a

Step 3 of Part 5 of Section 2.a

Step 4 of Part 5 of Section 2.a

Section 3.a of [9]

Section 3.a

Section 3.a

Section 3

Equation (3-2)

Equation (3-3)

Section 3.a

Section 3

Equation (3-4)

Step 3 in the proof of Lemma 3.5

in Section 3.a

Lemma 3.7 in Section 3.b

Lemma 3.8 in Section 3.c

Equation (3-33)

Equation (3-34) 
$N$ : the normal bundle to a submanifold

$N_{1}$ : a small radius disk subbundle of $N$

$e_{C}$ : a certain exponential map defined on $N_{1}$

$\mathrm{E}_{+}$

$\underline{E}$

$\mathrm{L}$

$\underline{\mathrm{L}}$

$\mathrm{O}$

$\underline{\mathrm{O}}$

$\mathrm{M}$

broken trajectory

$\left\{U_{C}\right\}_{(C, m) \in \vartheta},\left\{U_{\gamma-}\right\}_{\gamma \in \Xi_{\Sigma-}},\left\{U_{\gamma+}\right\}_{\gamma \in \Xi_{\Sigma+}}$, $U_{0}$

$\left(A^{*}, \psi^{*}\right)$ in the context of the proof of Theorem 1.2

$\mathfrak{s :}$ the tautological section of $\pi^{*} N \rightarrow N$

$\mathcal{E}$ : typically an end of a $J$-holomorphic curve $\mathcal{K}_{*}$

$\left(A^{\xi}, \psi^{\xi}\right)$

$\left(b^{\xi}, \eta^{\xi}, \phi^{\xi}\right)$

$\mathfrak{b}(\xi)$

$\mathfrak{t}^{\xi}$

$d_{M}$

$D$

$(\cdot) *(\cdot)$

$\mathfrak{h}(\xi)$

$\mathfrak{q}(\xi)$

$\Pi_{\xi}$

$\mathbb{H}_{\xi} \frac{\perp}{H_{\xi}} \frac{\perp}{\xi}=\left(1-\Pi_{\xi}\right) \mathbb{H}$

$\mathbb{L}$

$\mathfrak{t}_{\xi}:$ a homomorphism from $L$ to $L$

$\mathfrak{t}_{\xi}^{\dagger}$

$\left(c_{\xi}, \varsigma_{\xi}\right)$

$\theta_{1 \xi, r}$
Section 4.e

Section 4.e, Section 4.a of [9]

Section 4.e, Section 4.a of [9]

introduction to Section 5, Propo-

sition 5.1

Section 5.a

Section 5.a

Section 5.a

Section 5.b

Section 5.b

Section 5.d

Section 5.e

Section 6.b, Equation (5-3) of [9]

Part 3 of Section 6.b, Part 2 of Section 5.a of [9]

Part 3 of Section 6.b

Part 4 of Section 6.b

Section 6.c, Step 4 in Section 5.a of [9]

Section 6.c, Equation (5-15) in

Section 5.a of [9]

Section 6.c

Lemma 6.5

Section 6.c

Section 6.c

Equation (6-21), Section 6 of [9]

Equation (6-21), Section 6 of [9]

Section 6.d and Section 6.d of [9]

Section 6.d

Section 6.d, Part 6 in Section 7.a of [9]

Section 6.d

Section 6.d, Section 7.a of [9]

Section 6.d, Equation (6-9) of [9]

Section 6.d

Equation (6-23)

Equations (6-24), (6-57) of [9] 


$\mathfrak{k}_{\xi}$
$\|\cdot\|_{\mathcal{K}}$
$\|\cdot\|_{\mathcal{K}_{*}}$
$\|\cdot\|_{\mathcal{K}, \varepsilon}$
$\|\cdot\|_{\mathcal{K}_{1}^{2}}$
$\left.\theta=\left(\left(\theta_{C}\right)_{(C, m) \in \vartheta},\left(\theta_{\gamma-}\right)_{\gamma \in \Xi_{\Sigma-}}\right),\left(\theta_{\gamma+}\right)_{\gamma \in \Xi_{\Sigma+}}\right)$

$f_{0}, f_{1}, f_{+}, f_{-}$

$\mathfrak{o}_{k}$

$q_{\mathcal{E}}$

$z_{\mathcal{E}}$

$k_{\mathcal{E}}$

$\mathfrak{Z}_{\mathcal{E}}$
Equation (6-24)

Step 6 in the proof of Lemma 6.9, (5-13) of [9]

Step 6 in the proof of Lemma 6.9,

Step 4 of Section 5.a of [9]

Step 7 in the proof of Lemma 6.9

Section 6.e

Part 4 of the proof of Lemma 6.13, Equations (7-30), (7-31) and (7-34) of [9]

Equation (7-3)

Equation (7-9)

Step 1 of Part 2 in the proof of Lemma 7.7

Step 1 of Part 2 in the proof of Lemma 7.7, (4-1) of [9]

Step 1 of Part 2 in the proof of Lemma 7.7

Step 1 of Part 2 in the proof of Lemma 7.7

\section{References}

[1] F Bourgeois, Y Eliashberg, H Hofer, K Wysocki, E Zehnder, Compactness results in symplectic field theory, Geom. Topol. 7 (2003) 799-888 MR2026549

[2] M Hutchings, An index inequality for embedded pseudoholomorphic curves in symplectizations, J. Eur. Math. Soc. (JEMS) 4 (2002) 313-361 MR1941088

[3] M Hutchings, The embedded contact homology index revisited, from: "New perspectives and challenges in symplectic field theory", (M Abreu, F Lalonde, L Polterovich, editors), CRM Proc. Lecture Notes 49, Amer. Math. Soc. (2009) 263-297 MR2555941

[4] M Hutchings, M Sullivan, Rounding corners of polygons and the embedded contact homology of $T^{3}$, Geom. Topol. 10 (2006) 169-266 MR2207793

[5] P Kronheimer, T Mrowka, Monopoles and three-manifolds, New Math. Monogr. 10, Cambridge Univ. Press (2007) MR2388043

[6] CH Taubes, Seiberg-Witten and Gromov invariants for symplectic 4-manifolds, (R Wentworth, editor), First Int. Press Lecture Ser. 2, International Press, Somerville, MA (2000) MR1798809

[7] C H Taubes, The Seiberg-Witten equations and the Weinstein conjecture, Geom. Topol. 11 (2007) 2117-2202 MR2350473 
[8] CH Taubes, Embedded contact homology and Seiberg-Witten Floer cohomology I, Geom. Topol. 14 (2010) 2497-2581

[9] C H Taubes, Embedded contact homology and Seiberg-Witten Floer cohomology II, Geom. Topol. 14 (2010) 2583-2720

[10] C H Taubes, Embedded contact homology and Seiberg-Witten Floer cohomology III, Geom. Topol. 14 (2010) 2721-2817

Department of Mathematics, Harvard University

Cambridge, MA 02138, USA

chtaubes@math . harvard.edu

Proposed: Rob Kirby

Received: 15 November 2008

Seconded: Danny Calegari, Peter Ozsváth

Revised: 11 May 2010 